\title{
Quasi-Local Energy-Momentum and Angular Momentum in GR: A Review Article
}

\author{
László B. Szabados \\ Research Institute for Particle and Nuclear Physics \\ of the Hungarian Academy of Sciences \\ P. O. Box 49 \\ H-1525 Budapest 114 \\ Hungary \\ email: lbszab@rmki.kfki.hu \\ http://www.rmki.kfki.hu \\ Accepted on 27 February 2004 \\ Published on 16 March 2004 \\ http://www.livingreviews.org/lrr-2004-4 \\ Living Reviews in Relativity \\ Published by the Max Planck Institute for Gravitational Physics \\ Albert Einstein Institute, Germany
}

\begin{abstract}
The present status of the quasi-local mass-energy-momentum and angular momentum constructions in general relativity is reviewed. First the general ideas, concepts, and strategies, as well as the necessary tools to construct and analyze the quasi-local quantities are recalled. Then the various specific constructions and their properties (both successes and defects) are discussed. Finally, some of the (actual and potential) applications of the quasi-local concepts and specific constructions are briefly mentioned.

This review is based on the talks given at the Erwin Schrödinger Institute, Vienna, in July 1997, at the Universität Tübingen, in May 1998, and at the National Center for Theoretical Sciences in Hsinchu and at the National Central University, Chungli, Taiwan, in July 2000.
\end{abstract}

(C) Max Planck Society and the authors.

Further information on copyright is given at http://relativity.livingreviews . org/About/copyright.html

For permission to reproduce the article please contact livrev@aei.mpg.de. 


\section{How to cite this article}

Owing to the fact that a Living Reviews article can evolve over time, we recommend to cite the article as follows:

László B. Szabados,

"Quasi-Local Energy-Momentum and Angular Momentum in GR: A Review Article", Living Rev. Relativity, 7, (2004), 4. [Online Article]: cited [<date $>$ ], http://www.livingreviews.org/lrr-2004-4

The date given as $<$ date $>$ then uniquely identifies the version of the article you are referring to. 


\section{Article Revisions}

Living Reviews supports two different ways to keep its articles up-to-date:

Fast-track revision A fast-track revision provides the author with the opportunity to add short notices of current research results, trends and developments, or important publications to the article. A fast-track revision is refereed by the responsible subject editor. If an article has undergone a fast-track revision, a summary of changes will be listed here.

Major update A major update will include substantial changes and additions and is subject to full external refereeing. It is published with a new publication number.

For detailed documentation of an article's evolution, please refer always to the history document of the article's online version at http://www. livingreviews.org/lrr-2004-4.

01 Jun 2005: Recent developments of the field are included. 16 new references are added, minor improvements and corrections of the text are made at several points, and the bibliography is updated. The major changes are as follows:

Page 17: The discussion is changed, and a new paragraph is added. A new, quasi-local version of the holographic hypothesis is formulated, and it is explained how it works for physically important classical matter fields in Minkowski spacetime. Two references are added.

Page 21: Another first order spinor Lagrangian is included.

Page 36: Two paragraphs in Section 4.2.2 are rewritten, and a gap in the argumentation is filled.

Page 42: A new reference on the fall-off properties of self-interacting scalar fields in asymptotically anti-de-Sitter spacetimes is added.

Page 63: The small sphere expression of the Penrose energy in terms of the familiar conformal electric and magnetic parts of the Weyl curvature is given.

Page 69: A reference on the solutions of the Einstein-Yang-Mills equations that are characterized by the vanishing of the Dougan-Mason mass is added.

Page 78: A new paragraph about a new (non-scalar) second order Lagrangian, yielding the trace- $\chi$ action, and the relevant reference are added.

Page 84: A stronger energy positivity result for the Brown-York energy is included, and the reference is given.

Page 90: A stronger energy positivity result for the Kijowski-Liu-Yau energy is added, and the reference is given.

Page 94: A new reference is added.

Page 95: Section 11.3.2 is rewritten. A more detailed discussion of the Dirichlet and Neumann boundary conditions is given and new references are added.

Page 97: A new section (Section 11.3.3 in the new version) is opened for a part of the former Section 11.3.2 and for the recent 'main extrinsic curvature masses'. A new reference is added.

Page 101: A recent counter-example to the apparent horizon version of the Penrose inequality for non-time-symmetric data sets is mentioned, the discussion is improved and a reference is added.

Page 102: Two references on the existence of averaged trapped surfaces in non-spherically symmetric data sets are added. 


\section{Contents}

1 Introduction $\quad 9$

2 Energy-Momentum and Angular Momentum of Matter Fields $\quad 11$

2.1 Energy-momentum and angular momentum density of matter fields . . . . . . . . . 11

2.1.1 The symmetric energy-momentum tensor . . . . . . . . . . . . . . . 11

2.1.2 The canonical Noether current . . . . . . . . . . . . . . . . . . . 11

2.2 Quasi-local energy-momentum and angular momentum of the matter fields . . . . 13

2.2.1 The definition of the quasi-local quantities . . . . . . . . . . . . . 13

2.2.2 Hamiltonian introduction of the quasi-local quantities . . . . . . . . . . 15

2.2 .3 Properties of the quasi-local quantities . . . . . . . . . . . . . . . 16

2.2.4 Global energy-momenta and angular momenta . . . . . . . . . . . . . 17

2.2.5 Quasi-local radiative modes and a classical version of the holography for matter fields . . . . . . . . . . . . . . . . . . 17

3 On the Energy-Momentum and Angular Momentum of Gravitating Systems

3.1 On the gravitational energy-momentum and angular momentum density: The diff-

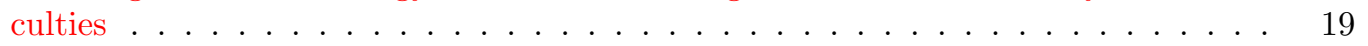

3.1.1 The root of the difficulties . . . . . . . . . . . . . . . . . 19

3.1 .2 Pseudotensors. . . . . . . . . . . . . . . . . . . . 20

3.1.3 Strategies to avoid pseudotensors I: Background metrics/connections . . . . 21

3.1.4 Strategies to avoid pseudotensors II: The tetrad formalism . . . . . . . . . . 21

3.1.5 Strategies to avoid pseudotensors III: Higher derivative currents . . . . . . 22

3.2 On the global energy-momentum and angular momentum of gravitating systems:

The successes . . . . . . . . . . . . . . . . . . . . . . 22

3.2.1 Spatial infinity: Energy-momentum _ . . . . . . . . . . . . . . 22

3.2 .2 Spatial infinity: Angular momentum . . . . . . . . . . . . . . . . 24

3.2 .3 Null infinity: Energy-momentum . . . . . . . . . . . . . . . . . . 25

3.2 .4 Null infinity: Angular momentum _. . . . . . . . . . . . . . . 26

3.3 The necessity of quasi-locality for the observables in general relativity . . . . . . 28

3.3.1 Non-locality of the gravitational energy-momentum and angular momentum 28

3.3.2 Domains for quasi-local quantities . . . . . . . . . . . . . . . . 28

3.3.3 Strategies to construct quasi-local quantities . . . . . . . . . . . . . . . 29

4 Tools to Construct and Analyze the Quasi-Local Quantities 31

4.1 The geometry of spacelike 2 -surfaces . . . . . . . . . . . . . . . . . . 31

4.1.1 The Lorentzian vector bundle . . . . . . . . . . . . . . . . . . . . . . 31

4.1 .2 Connections . . . . . . . . . . . . . . . . . . . . . 31

4.1 .3 Convexity conditions . . . . . . . . . . . . . . . . . . . . 32

4.1 .4 The spinor bundle $\ldots \ldots \ldots \ldots \ldots \ldots$

4.1 .5 Curvature identities . . . . . . . . . . . . . . . . . . . 33

4.1 .6 The GHP formalism . . . . . . . . . . . . . . . . . . 33

4.1.7 Irreducible parts of the derivative operators . . . . . . . . . . . . . . . 34

4.1.8 SO (1,1)-connection 1-form versus anholonomicity . . . . . . . . . . 34

4.2 Standard situations to evaluate the quasi-local quantities . . . . . . . . . . . 35

4.2 .1 Round spheres . . . . . . . . . . . . . . . . . . . . 35

4.2 .2 Small surfaces . . . . . . . . . . . . . . . . . . . . . 36

$4.2 .3 \quad$ Large spheres near the spatial infinity . . . . . . . . . . . . . . . . . 39

4.2 .4 Large spheres near null infinity . . . . . . . . . . . . . . . . . . . . . . . . . . . . . .

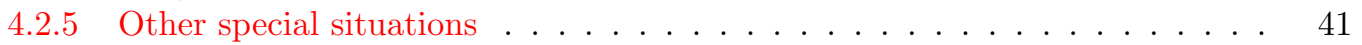


4.3 On lists of criteria of reasonableness of the quasi-local quantities . . . . . . . . . . . 42



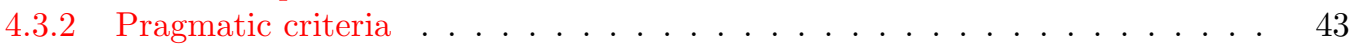

4.3.3 Incompatibility of certain 'natural' expectations . . . . . . . . . 45

5 The Bartnik Mass and its Modifications $\quad 46$

5.1 The Bartnik mass . . . . . . . . . . . . . . . . . . . . . . . . . 46

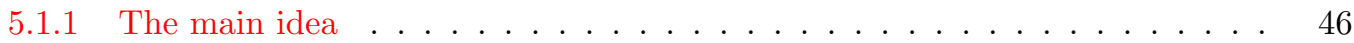

5.1 .2 The main properties of $m_{\mathrm{B}}(\Sigma) \ldots \ldots \ldots \ldots$. . . . . . . . . . . . . . . . . . . . . . . . . . 47

5.1 .3 The computability of the Bartnik mass . . . . . . . . . . . . . . . 48

5.2 Bray's modifications . . . . . . . . . . . . . . . . . . . . . 48

6 The Hawking Energy and its Modifications $\quad \mathbf{5 0}$

6.1 The Hawking energy . . . . . . . . . . . . . . . . . . . . . . . 50 50

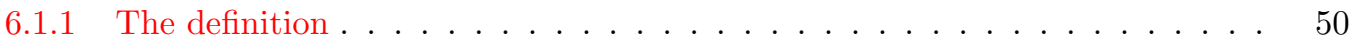

6.1 .2 The Hawking energy for spheres . . . . . . . . . . . . . . . 50

6.1.3 Positivity and monotonicity properties . . . . . . . . . . . . . 51

6.1 .4 Two generalizations . . . . . . . . . . . . . . . . . . . . . . 52

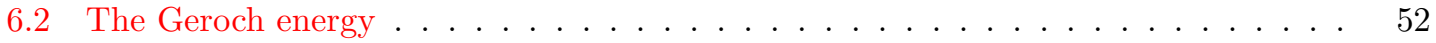

6.2 .1 The definition . . . . . . . . . . . . . . . . . . 52

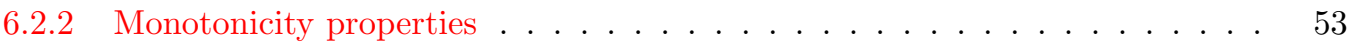

6.3 The Hayward energy . . . . . . . . . . . . . . . . . . . . . 53

7 Penrose's Quasi-Local Energy-Momentum and Angular Momentum 55

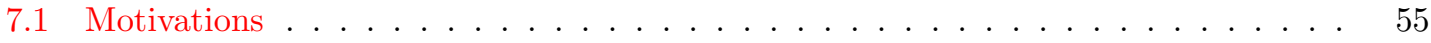

7.1.1 How do the twistors emerge? . . . . . . . . . . . . . . 55

7.1 .2 Twistor space and the kinematical twistor . . . . . . . . . . . . . . 56

7.2 The original construction for curved spacetimes . . . . . . . . . . . . . . 58

7.2.1 2-surface twistors and the kinematical twistor . . . . . . . . . . . 58

7.2.2 The Hamiltonian interpretation of the kinematical twistor . . . . . . . . . 58

7.2.3 The Hermitian scalar product and the infinity twistor . . . . . . . . . 59

7.2 .4 The various limits . . . . . . . . . . . . . . . . . . . 60

7.2 .5 The quasi-local mass of specific 2 -surfaces . . . . . . . . . . . . . . . 61

7.2 .6 Small surfaces . . . . . . . . . . . . . . . . . . . . 63

7.3 The modified constructions . . . . . . . . . . . . . . . . . . 63

7.3.1 The 'improved' construction with the determinant . . . . . . . . . . 63

7.3.2 Modification through Tod's expression . . . . . . . . . . . . . . 64

7.3.3 Mason's suggestions ...................... 64

8 Approaches Based on the Nester-Witten 2-Form $\quad \mathbf{6 5}$

8.1 The Ludvigsen-Vickers construction . . . . . . . . . . . . . . . . . . 66 66

8.1 The definition . . . . . . . . . . . . . . . . 66

8.1.2 Remarks on the validity of the construction . . . . . . . . . . . . 66

8.1.3 Monotonicity, mass-positivity and the various limits . . . . . . . . . . . 67

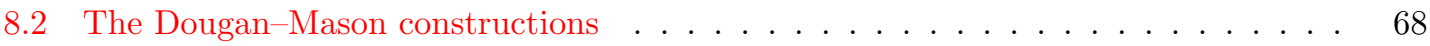

8.2.1 Holomorphic/anti-holomorphic spinor fields . . . . . . . . . . . . 68

8.2 .2 The genericity of the generic 2 -surfaces . . . . . . . . . . . . . 68

8.2 .3 Positivity properties . . . . . . . . . . . . . . . . 69

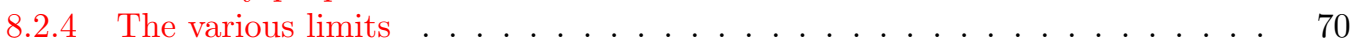

8.3 A specific construction for the Kerr spacetime . . . . . . . . . . . . . . 71 
9 Quasi-Local Spin-Angular Momentum

9.1 The Ludvigsen-Vickers angular momentum . . . . . . . . . . . . . . . . . . . . . 73

9.2 Holomorphic/anti-holomorphic spin-angular momenta . . . . . . . . . . . . . 74

9.3 A specific construction for the Kerr spacetime . . . . . . . . . . . . . . . . . . . 75

10 The Hamilton-Jacobi Method $\quad \mathbf{7 6}$

10.1 The Brown-York expression . . . . . . . . . . . . . . . . . . 76

10.1.1 The main idea . . . . . . . . . . . . . . . . . 76

10.1.2 The variation of the action and the surface stress-energy tensor . . . . . . 77

10.1.3 The general form of the Brown-York quasi-local energy . . . . . . . . . 78

10.1.4 Further properties of the general expressions . . . . . . . . . . . . 79

10.1.5 The Hamiltonians . . . . . . . . . . . . . . . . . . . . . . 81

10.1.6 The flat space and light cone references . . . . . . . . . . . . . 82

10.1.7 Further properties and the various limits . . . . . . . . . . . 83

10.1.8 Other prescriptions for the reference configuration . . . . . . . . 86

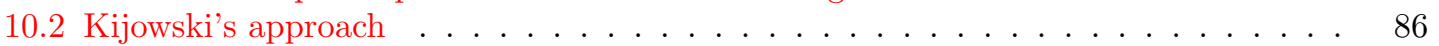

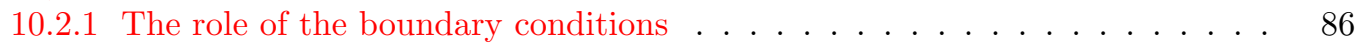

10.2.2 The analysis of the Hilbert action and the quasi-local internal and free energies 86

10.3 The expression of Epp . . . . . . . . . . . . . . . . . . . . . 88

10.3.1 The general form of Epp's expression . . . . . . . . . . . . . . 88

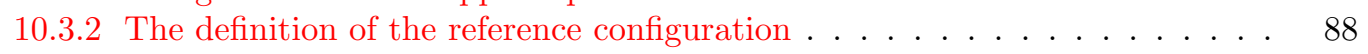

10.3 .3 The various limits . . . . . . . . . . . . . . . . . . . . . 89

10.4 The expression of Liu and Yau $\ldots \ldots \ldots \ldots \ldots$

10.4.1 The Liu-Yau definition . . . . . . . . . . . . . . . . . . . . 89

10.4.2 The main properties of $E_{\mathrm{KLY}}(\mathcal{S}) \ldots \ldots \ldots \ldots$

11 Towards a Full Hamiltonian Approach $\quad 92$

11.1 The $3+1$ approaches . . . . . . . . . . . . . . . . . . . . . 92

11.1.1 The 2-surface observables . . . . . . . . . . . . . . . . . . . 92

11.2 Approaches based on the double-null foliations . . . . . . . . . . . . . . . . 94

11.2.1 The $2+2$ decomposition . . . . . . . . . . . . . . . . . . 94

11.2.2 The $2+2$ quasi-localization of the Bondi-Sachs mass-loss . . . . . . . . 94

11.3 The covariant approach . . . . . . . . . . . . . . . . . . . 95

11.3.1 The covariant phase space methods . . . . . . . . . . . . . . 95

11.3.2 Covariant quasi-local Hamiltonians with explicit reference configurations . . 95

11.3.3 Covariant quasi-local Hamiltonians with general reference terms . . . . . . 97

11.3.4 Pseudotensors and quasi-local quantities . . . . . . . . . . . . . . . 98

12 Constructions for Special Spacetimes $\quad 99$

12.1 The Komar integral for spacetimes with Killing vectors . . . . . . . . . . . . . . 99

12.2 The effective mass of Kulkarni, Chellathurai, and Dadhich for the Kerr spacetime . 99

12.3 The Katz-Lynden-Bell-Israel energy for static spacetimes . . . . . . . . . . . . . 99

13 Applications in General Relativity 101

13.1 Calculation of tidal heating . . . . . . . . . . . . . . . . . . . 101

13.2 Geometric inequalities for black holes . . . . . . . . . . . . . . . . . . . . . 101

13.2.1 On the Penrose inequality . . . . . . . . . . . . . . . . . . . . 101

13.2.2 On the hoop conjecture . . . . . . . . . . . . . . . . . 102

13.3 Quasi-local laws of black hole dynamics . . . . . . . . . . . . . . . . . . . 103

13.3.1 Quasi-local thermodynamics of black holes . . . . . . . . . . . . . 103

13.3.2 On the isolated horizons . . . . . . . . . . . . . . . . . . . . 104 
13.4 Entropy bounds . . . . . . . . . . . . . . . . . . . . . . . . . . 105

13.4.1 On Bekenstein's bounds for the entropy . . . . . . . . . . . . . . 105

13.4.2 On the holographic hypothesis . . . . . . . . . . . . . . . 105

13.5 Quasi-local radiative modes of GR . . . . . . . . . . . . . . . 106

14 Summary: Achievements, Difficulties, and Open Issues 107

14.1 On the Bartnik mass and the Hawking energy . . . . . . . . . . . . . . . . 107

14.2 On the Penrose mass . . . . . . . . . . . . . . . . . . . . . . . . 108

14.3 On the Dougan-Mason energy-momenta and the holomorphic/anti-holomorphic spin angular momenta . . . . . . . . . . . . . . . . . . . . . . . . 108

14.4 On the Brown-York-type expressions . . . . . . . . . . . . . . . . . . . . 109

15 Acknowledgments $\quad 112$

$\begin{array}{ll}\text { References } & 140\end{array}$ 



\section{Introduction}

During the last 25 years one of the greatest achievements in classical general relativity is certainly the proof of the positivity of the total gravitational energy, both at spatial and null infinity. It is precisely its positivity that makes this notion not only important (because of its theoretical significance), but a useful tool as well in the everyday practice of working relativists. This success inspired the more ambitious claim to associate energy (or rather energy-momentum and, ultimately, angular momentum too) to extended but finite spacetime domains, i.e. at the quasi-local level. Obviously, the quasi-local quantities could provide a more detailed characterization of the states of the gravitational 'field' than the global ones, so they (together with more general quasi-local observables) would be interesting in their own right.

Moreover, finding an appropriate notion of energy-momentum and angular momentum would be important from the point of view of applications as well. For example, they may play a central role in the proof of the full Penrose inequality (as they have already played in the proof of the Riemannian version of this inequality). The correct, ultimate formulation of black hole thermodynamics should probably be based on quasi-locally defined internal energy, entropy, angular momentum etc. In numerical calculations conserved quantities (or at least those for which balance equations can be derived) are used to control the errors. However, in such calculations all the domains are finite, i.e. quasi-local. Therefore, a solid theoretical foundation of the quasi-local conserved quantities is needed.

However, contrary to the high expectations of the eighties, finding an appropriate quasi-local notion of energy-momentum has proven to be surprisingly difficult. Nowadays, the state of the art is typically postmodern: Although there are several promising and useful suggestions, we have not only no ultimate, generally accepted expression for the energy-momentum and especially for the angular momentum, but there is no consensus in the relativity community even on general questions (for example, what should we mean e.g. by energy-momentum: Only a general expression containing arbitrary functions, or rather a definite one free of any ambiguities, even of additive constants), or on the list of the criteria of reasonableness of such expressions. The various suggestions are based on different philosophies, approaches and give different results in the same situation. Apparently, the ideas and successes of one construction have only very little influence on other constructions.

The aim of the present paper is therefore twofold. First, to collect and review the various specific suggestions, and, second, to stimulate the interaction between the different approaches by clarifying the general, potentially common points, issues, questions. Thus we wanted to write not only a 'who-did-what' review, but primarily we would like to concentrate on the understanding of the basic questions (such as why should the gravitational energy-momentum and angular momentum, or, more generally, any observable of the gravitational 'field', be necessarily quasi-local) and ideas behind the various specific constructions. Consequently, one-third of the present review is devoted to these general questions. We review the specific constructions and their properties only in the second part, and in the third part we discuss very briefly some (potential) applications of the quasi-local quantities. Although this paper is basically a review of known and published results, we believe that it contains several new elements, observations, suggestions etc.

Surprisingly enough, most of the ideas and concepts that appear in connection with the gravitational energy-momentum and angular momentum can be introduced in (and hence can be understood from) the theory of matter fields in Minkowski spacetime. Thus, in Section 2.1, we review the Belinfante--Rosenfeld procedure that we will apply to gravity in Section 3, introduce the notion of quasi-local energy-momentum and angular momentum of the matter fields and discuss their properties. The philosophy of quasi-locality in general relativity will be demonstrated in Minkowski spacetime where the energy-momentum and angular momentum of the matter fields are treated quasi-locally. Then we turn to the difficulties of gravitational energy-momentum and angular mo-

Living Reviews in Relativity

http://www. livingreviews. org/Irr-2004-4 
mentum, and we clarify why the gravitational observables should necessarily be quasi-local. The tools needed to construct and analyze the quasi-local quantities are reviewed in the fourth section. This closes the first, the general part of the review.

The second part is devoted to the discussion of the specific constructions (Sections 5-12). Since most of the suggestions are constructions, they cannot be given as a short mathematical definition. Moreover, there are important physical ideas behind them, without which the constructions may appear ad hoc. Thus we always try to explain these physical pictures, the motivations and interpretations. Although the present paper is intended to be a non-technical review, the explicit mathematical definitions of the various specific constructions will always be given. Then the properties and the applications are usually summarized only in a nutshell. Sometimes we give a review on technical aspects too, without which it would be difficult to understand even some of the conceptual issues. The list of references connected with this second part is intended to be complete. We apologize to all those whose results were accidentally left out.

The list of the (actual and potential) applications of the quasi-local quantities, discussed in Section 13, is far from being complete, and might be a little bit subjective. Here we consider the calculation of gravitational energy transfer, applications in black hole physics and a quasi-local characterization of the $p p$-wave metrics. We close this paper with a discussion of the successes and deficiencies of the general and (potentially) viable constructions. In contrast to the positivistic style of Sections 5-12, Section 14 (as well as the choice for the matter of Sections 2, 3, and refsec-4) reflects our own personal interest and view of the subject.

The theory of quasi-local observables in general relativity is far from being complete. The most important open problem is still the trivial one: 'Find quasi-local energy-momentum and angular momentum expressions satisfying the points of the lists of Section 4.3'. Several specific open questions in connection with the specific definitions are raised both in the corresponding sections and in Section 14, which could be worked out even by graduate students. On the other hand, any of their application to solve physical/geometrical problems (e.g. to some mentioned in Section 13) would be a real success.

In the present paper we adopt the abstract index formalism. The signature of the spacetime metric $g_{a b}$ is -2 , and the curvature and Ricci tensors and the curvature scalar of the covariant derivative $\nabla_{a}$ are defined by $\left(\nabla_{c} \nabla_{d}-\nabla_{d} \nabla_{c}\right) X^{a}:=-R_{b c d}^{a} X^{b}, R_{b d}:=R_{b a d}^{a}$ and $R:=R_{b d} g^{b d}$, respectively. Hence Einstein's equations take the form $G_{a b}+\lambda g_{a b}:=R_{a b}-\frac{1}{2} R g_{a b}+\lambda g_{a b}=$ $-8 \pi G T_{a b}$, where $G$ is Newton's gravitational constant and $\lambda$ is the cosmological constant (and the speed of light is $c=1$ ). However, apart from special cases stated explicitly, the cosmological constant will be assumed to be vanishing, and in Sections 13.3 and 13.4 we use the traditional cgs system.

Living Reviews in Relativity

http://www. livingreviews .org/lrr-2004-4 


\section{Energy-Momentum and Angular Momentum of Matter Fields}

\subsection{Energy-momentum and angular momentum density of matter fields}

\subsubsection{The symmetric energy-momentum tensor}

It is a widely accepted view (appearing e.g. in excellent, standard textbooks on general relativity, too) that the canonical energy-momentum and spin tensors are well-defined and have relevance only in flat spacetime, and hence usually are underestimated and abandoned. However, it is only the analog of these canonical quantities that can be associated with gravity itself. Thus first we introduce these quantities for the matter fields in a general curved spacetime.

To specify the state of the matter fields operationally two kinds of devices are needed: The first measures the value of the fields, while the other measures the spatio-temporal location of the first. Correspondingly, the fields on the manifold $M$ of events can be grouped into two sharply distinguished classes: The first contains the matter field variables, e.g. finitely many $(r, s)$-type tensor fields $\Phi_{N} \begin{gathered}a_{1} \ldots a_{r} \\ b_{1} \ldots b_{s}\end{gathered}$, whilst the other contains the fields specifying the spacetime geometry, i.e. the metric $g_{a b}$ in Einstein's theory. Suppose that the dynamics of the matter fields is governed by Hamilton's principle specified by a Lagrangian $L_{\mathrm{m}}=L_{\mathrm{m}}\left(g^{a b}, \Phi_{N}, \nabla_{e} \Phi_{N}, \ldots, \nabla_{e_{1}} \ldots \nabla_{e_{k}} \Phi_{N}\right)$ : If $I_{\mathrm{m}}\left[g^{a b}, \Phi_{N}\right]$ is the volume integral of $L_{\mathrm{m}}$ on some open domain $D$ with compact closure then the equations of motion are $E^{N b_{\ldots} \ldots}:=\delta I_{\mathrm{m}} / \delta \Phi_{N_{b \ldots}^{a} \ldots}^{a \ldots}=\sum_{n=0}^{k}(-)^{n} \nabla_{e_{n}} \ldots \nabla_{e_{1}}\left(\partial L_{\mathrm{m}} / \partial\left(\nabla_{e_{1}} \ldots \nabla_{e_{n}} \Phi_{N_{b} \ldots}^{a \ldots}\right)\right)=$ 0 , the Euler-Lagrange equations. The symmetric (or dynamical) energy-momentum tensor is defined (and is given explicitly) by

$$
T_{a b}:=\frac{2}{\sqrt{|g|}} \frac{\delta I_{\mathrm{m}}}{\delta g^{a b}}=2 \frac{\partial L_{\mathrm{m}}}{\partial g^{a b}}-L_{\mathrm{m}} g_{a b}+\frac{1}{2} \nabla^{e}\left(\sigma_{a b e}+\sigma_{b a e}-\sigma_{a e b}-\sigma_{b e a}-\sigma_{e a b}-\sigma_{e b a}\right)
$$

where we introduced the so-called canonical spin tensor

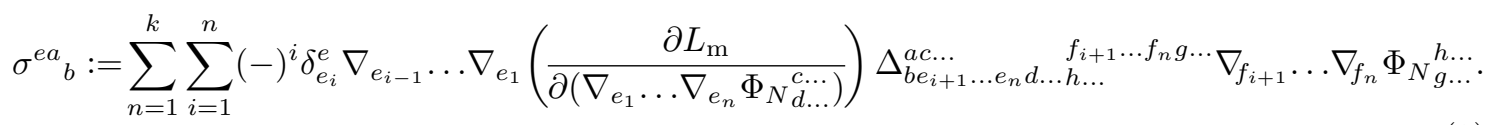

(The terminology will be justified in the next Section 2.2.) Here $\Delta_{d b_{1} \ldots b_{q} e_{1} \ldots e_{p}}^{c a_{1} \ldots a_{1} f_{1} \ldots f_{q}}$ is the $(p+q+1, p+$ $q+1)$-type invariant tensor, built from the Kronecker deltas, appearing naturally in the expression of the Lie derivative of the $(p, q)$-type tensor fields in terms of the torsion free covariant derivatives: $\mathrm{E}_{\mathbf{K}} \Phi_{b \ldots}^{a \ldots}=\nabla_{\mathbf{K}} \Phi_{b \ldots}^{a \ldots}-\nabla_{c} K^{d} \Delta_{d b \ldots e \ldots}^{c a \ldots f \ldots} \Phi_{f \ldots}^{e \ldots}$. (For the general idea of deriving $T_{a b}$ and Equation (2), see e.g. Section 3 of [175].)

\subsubsection{The canonical Noether current}

Suppose that the Lagrangian is weakly diffeomorphism invariant in the sense that for any vector field $K^{a}$ and the corresponding local 1-parameter family of diffeomorphisms $\phi_{t}$ one has

$$
\left(\phi_{t}^{*} L_{\mathrm{m}}\right)\left(g^{a b}, \Phi_{N}, \nabla_{e} \Phi_{N}, \ldots\right)-L_{\mathrm{m}}\left(\phi_{t}^{*} g^{a b}, \phi_{t}^{*} \Phi_{N}, \phi_{t}^{*} \nabla_{e} \Phi_{N}, \ldots\right)=\nabla_{e} B_{t}^{e}
$$

for some 1-parameter family of vector fields $B_{t}^{e}=B_{t}^{e}\left(g^{a b}, \Phi_{N}, \ldots\right)$. ( $L_{\mathrm{m}}$ is called diffeomorphism invariant if $\nabla_{e} B_{t}^{e}=0$, e.g. when $L_{\mathrm{m}}$ is a scalar.) Let $K^{a}$ be any smooth vector field on $M$. Then, calculating the divergence $\nabla_{a}\left(L_{\mathrm{m}} K^{a}\right)$ to determine the rate of change of the action functional $I_{\mathrm{m}}$ along the integral curves of $K^{a}$, by a tedious but straightforward computation one can derive the so-called Noether identity: $E^{N{ }_{a \ldots} \ldots} \mathrm{E}_{\mathbf{K}} \Phi_{N}^{a \ldots \ldots}+\frac{1}{2} T_{a b} \mathrm{E}_{\mathbf{K}} g^{a b}+\nabla_{e} C^{e}[\mathbf{K}]=0$, where $\mathrm{E}_{\mathbf{K}}$ denotes the 
Lie derivative along $K^{a}$, and $C^{a}[\mathbf{K}]$, the so-called Noether current, is given explicitly by

$$
C^{e}[\mathbf{K}]=\dot{B}^{e}+\theta^{e a} K_{a}+\left(\sigma^{e[a b]}+\sigma^{a[b e]}+\sigma^{b[a e]}\right) \nabla_{a} K_{b} .
$$

Here $\dot{B}^{e}$ is the derivative of $B_{t}^{e}$ with respect to $t$ at $t=0$, which may depend on $K_{a}$ and its derivatives, and $\theta^{a}$, the so-called canonical energy-momentum tensor, is defined by

$$
\theta_{b}^{a}:=-L_{\mathrm{m}} \delta_{b}^{a}-\sum_{n=1}^{k} \sum_{i=1}^{n}(-)^{i} \delta_{e_{i}}^{a} \nabla_{e_{i-1}} \ldots \nabla_{e_{1}}\left(\frac{\partial L_{\mathrm{m}}}{\partial\left(\nabla_{e_{1}} \ldots \nabla_{e_{n}} \Phi_{N_{d \ldots}}^{c \ldots}\right)}\right) \nabla_{b} \nabla_{e_{i+1}} \ldots \nabla_{e_{n}} \Phi_{N_{d \ldots} \ldots}^{c \ldots}
$$

Note that, apart from the term $\dot{B}^{e}$, the current $C^{e}[\mathbf{K}]$ does not depend on higher than the first derivative of $K^{a}$, and the canonical energy-momentum and spin tensors could be introduced as the coefficients of $K_{a}$ and its first derivative, respectively, in $C^{e}[\mathbf{K}]$. (For the original introduction of these concepts, see $[56,57,323]$. If the torsion $\Theta^{c}{ }_{a b}$ is not vanishing, then in the Noether identity there is a further term, $\frac{1}{2} S^{a b}{ }_{c} \mathrm{E}_{\mathbf{K}} \Theta^{c}{ }_{a b}$, where the so-called dynamical spin tensor $S^{a b}{ }_{c}$ is defined by $\sqrt{|g|} S^{a b}{ }_{c}:=2 \delta I_{\mathrm{m}} / \delta \Theta^{c}{ }_{a b}$, and the Noether current has a slightly different structure [193, 194].) Obviously, $C^{e}[\mathbf{K}]$ is not uniquely determined by the Noether identity, because that contains only its divergence, and any identically conserved current may be added to it. In fact, $B_{t}^{e}$ may be chosen to be an arbitrary non-zero (but divergence free) vector field even for diffeomorphism invariant Lagrangians. Thus, to be more precise, if $\dot{B}^{e}=0$, then we call the specific combination (3) the canonical Noether current. Other choices for the Noether current may contain higher derivatives of $K^{a}$, too (see e.g. [228]), but there is a specific one containing $K^{a}$ algebraically (see the Points 3 and 4 below). However, $C^{a}[\mathbf{K}]$ is sensitive to total divergences added to the Lagrangian, and, if the matter fields have gauge freedom, then in general it is not gauge invariant even if the Lagrangian is. On the other hand, $T^{a b}$ is gauge invariant and is independent of total divergences added to $L_{\mathrm{m}}$ because it is the variational derivative of the gauge invariant action with respect to the metric. Provided the field equations are satisfied, the Noether identity implies [56, 57, 323, 193, 194] that

1. $\nabla_{a} T^{a b}=0$,

2. $T^{a b}=\theta^{a b}+\nabla_{c}\left(\sigma^{c[a b]}+\sigma^{a[b c]}+\sigma^{b[a c]}\right)$,

3. $C^{a}[\mathbf{K}]=T^{a b} K_{b}+\nabla_{c}\left(\left(\sigma^{a[c b]}-\sigma^{c[a b]}-\sigma^{b[a c]}\right) K_{b}\right)$, where the second term on the right is an identically conserved (i.e. divergence free) current, and

4. $C^{a}[\mathbf{K}]$ is conserved if $K^{a}$ is a Killing vector.

Hence $T^{a b} K_{b}$ is also conserved and can equally be considered as a Noether current. (For a formally different, but essentially equivalent introduction of the Noether current and identity, see [389, 215, 141].)

The interpretation of the conserved currents $C^{a}[\mathbf{K}]$ and $T^{a b} K_{b}$ depends on the nature of the Killing vector $K^{a}$. In Minkowski spacetime the 10-dimensional Lie algebra $\mathrm{K}$ of the Killing vectors is well known to split to the semidirect sum of a 4-dimensional commutative ideal, $\mathrm{T}$, and the quotient $\mathrm{K} / \mathrm{T}$, where the latter is isomorphic to $s o(1,3)$. The ideal $\mathrm{T}$ is spanned by the constant Killing vectors, in which a constant orthonormal frame field $\left\{E_{a}^{a}\right\}$ on $M, \underline{a}=0, \ldots, 3$, forms a basis. (Thus the underlined Roman indices $\underline{a}, \underline{b}, \ldots$ are concrete, name indices.) By $g_{a b} E_{a}^{a} E_{b}^{b}=$ $\eta_{\underline{a} \underline{b}}:=\operatorname{diag}(1,-1,-1,-1)$ the ideal $\mathrm{T}$ inherits a natural Lorentzian vector space structure. Having chosen an origin $o \in M$, the quotient $\mathrm{K} / \mathrm{T}$ can be identified as the Lie algebra $\mathrm{R}_{o}$ of the boostrotation Killing vectors that vanish at $o$. Thus $\mathrm{K}$ has a ' $4+6$ ' decomposition into translations and boost-rotations, where the translations are canonically defined but the boost-rotations depend on the choice of the origin $o \in M$. In the coordinate system $\left\{x^{\underline{a}}\right\}$ adapted to $\left\{E_{\underline{a}}^{a}\right\}$ (i.e. for which the

Living Reviews in Relativity

http://www. livingreviews.org//rr-2004-4 
1-form basis dual to $\left\{E_{a}^{a}\right\}$ has the form $\vartheta_{a}^{a}=\nabla_{a} x^{\underline{a}}$ ) the general form of the Killing vectors (or rather 1-forms) is $K_{a}=T_{\underline{a}} \vartheta_{a}^{\underline{a}}+M_{\underline{a} \underline{b}}\left(x^{\underline{a}} \vartheta_{\bar{a}}^{\underline{b}}-x^{\underline{b}} \vartheta_{a}^{\underline{a}}\right)$ for some constants $T_{\underline{a}}$ and $M_{\underline{a}} \underline{\underline{b}}=-M_{\underline{b} \underline{a}}$. Then the corresponding canonical Noether current is $C^{e}[\mathbf{K}]=E_{\underline{e}}^{e}\left(\theta^{\underline{e} \underline{a}} T_{\underline{a}}-\left(\theta^{\underline{e}} \underline{a} x^{\underline{\underline{b}}}-\theta^{\underline{\underline{e}} \underline{b}} x^{\underline{\underline{a}}}-\right.\right.$ $\left.\left.2 \sigma^{\underline{e}}[\underline{a} \underline{b}]\right) M_{\underline{a}} \underline{b}\right)$, and the coefficients of the translation and the boost-rotation parameters $T_{\underline{a}}$ and $M_{\underline{a} \underline{b}}$ are interpreted as the density of the energy-momentum and the sum of the orbital and spin angular momenta, respectively. Since, however, the difference $C^{a}[\mathbf{K}]-T^{a b} K_{b}$ is identically conserved and $T^{a b} K_{b}$ has more advantageous properties, it is $T^{a b} K_{b}$ that is used to represent the energy-momentum and angular momentum density of the matter fields.

Since in the de-Sitter and anti-de-Sitter spacetimes the (ten dimensional) Lie algebra of the Killing vector fields, $s o(1,4)$ and $s o(2,3)$, respectively, are semisimple, there is no such natural notion of translations, and hence no natural ' $4+6$ ' decomposition of the ten conserved currents into energy-momentum and (relativistic) angular momentum density.

\subsection{Quasi-local energy-momentum and angular momentum of the mat- ter fields}

In the next Section 3 we will see that well-defined (i.e. gauge invariant) energy-momentum and angular momentum density cannot be associated with the gravitational 'field', and if we want to talk not only about global gravitational energy-momentum and angular momentum, then these quantities must be assigned to extended but finite spacetime domains.

In the light of modern quantum field theoretical investigations it has become clear that all physical observables should be associated with extended but finite spacetime domains [169, 168]. Thus observables are always associated with open subsets of spacetime whose closure is compact, i.e. they are quasi-local. Quantities associated with spacetime points or with the whole spacetime are not observable in this sense. In particular, global quantities, such as the total energy or electric charge, should be considered as the limit of quasi-locally defined quantities. Thus the idea of quasilocality is not new in physics. Although apparently in classical non-gravitational physics this is not obligatory, we adopt this view in talking about energy-momentum and angular momentum even of classical matter fields in Minkowski spacetime. Originally the introduction of these quasilocal quantities was motivated by the analogous gravitational quasi-local quantities [354, 358]. Since, however, many of the basic concepts and ideas behind the various gravitational quasi-local energy-momentum and angular momentum definitions can be understood from the analogous nongravitational quantities in Minkowski spacetime, we devote the present section to the discussion of them and their properties.

\subsubsection{The definition of the quasi-local quantities}

To define the quasi-local conserved quantities in Minkowski spacetime, first observe that for any Killing vector $K^{a} \in \mathrm{K}$ the 3 -form $\omega_{a b c}:=K_{e} T^{e f} \varepsilon_{f a b c}$ is closed, and hence, by the triviality of the third de Rham cohomology class, $H^{3}\left(\mathbb{R}^{4}\right)=0$, it is exact: For some 2-form $\cup[\mathbf{K}]_{a b}$ we have $K_{e} T^{e f} \varepsilon_{f a b c}=3 \nabla_{[a} \cup[\mathbf{K}]_{b c]} . \vee^{c d}:=-\frac{1}{2} \cup[\mathbf{K}]_{a b} \varepsilon^{a b c d}$ may be called a 'superpotential' for the conserved current 3 -form $\omega_{a b c}$. (However, note that while the superpotential for the gravitational energy-momentum expressions of the next Section 3 is a local function of the general field variables, the existence of this 'superpotential' is a consequence of the field equations and the Killing nature of the vector field $K^{a}$. The existence of globally defined superpotentials that are local functions of the field variables can be proven even without using the Poincaré lemma [388].) If $\tilde{\cup}[\mathbf{K}]_{a b}$ is (the dual of) another superpotential for the same current $\omega_{a b c}$, then by $\nabla_{[a}\left(\cup[\mathbf{K}]_{b c]}-\tilde{\cup}[\mathbf{K}]_{b c]}\right)=0$ and $H^{2}\left(\mathbb{R}^{4}\right)=0$ the dual superpotential is unique up to the addition of an exact 2-form. If therefore $\mathcal{S}$ is any closed orientable spacelike 2-surface in the Minkowski spacetime then the integral of $\cup[\mathbf{K}]_{a b}$ on $\mathcal{S}$ is free from this ambiguity. Thus if $\Sigma$ is any smooth compact spacelike hypersurface with 
smooth 2-boundary $\mathcal{S}$, then

$$
Q_{\mathcal{S}}[\mathbf{K}]:=\frac{1}{2} \oint_{\mathcal{S}} \cup[\mathbf{K}]_{a b}=\int_{\Sigma} K_{e} T^{e f} \frac{1}{3 !} \varepsilon_{f a b c}
$$

depends only on $\mathcal{S}$. Hence it is independent of the actual Cauchy surface $\Sigma$ of the domain of dependence $D(\Sigma)$ because all the spacelike Cauchy surfaces for $D(\Sigma)$ have the same common boundary $\mathcal{S}$. Thus $Q_{\mathcal{S}}[\mathbf{K}]$ can equivalently be interpreted as being associated with the whole domain of dependence $D(\Sigma)$, and hence quasi-local in the sense of $[169,168]$ above. It defines the linear maps $P_{\mathcal{S}}: \mathrm{T} \rightarrow \mathbb{R}$ and $J_{\mathcal{S}}: \mathrm{R}_{o} \rightarrow \mathbb{R}$ by $Q_{\mathcal{S}}[\mathbf{K}]=: T_{\underline{a}} P_{\mathcal{S}}^{\underline{a}}+M_{\underline{a}} \underline{b} J_{\mathcal{S}} \underline{\underline{b}}$, i.e. they are elements of the corresponding dual spaces. Under Lorentz rotations of the Cartesian coordinates, $P \overline{\mathcal{S}}_{\mathcal{S}}$ and $J_{\mathcal{S}} \underline{a}$ transform as a Lorentz vector and anti-symmetric tensor, respectively, whilst under the translation $x^{\underline{a}} \mapsto x^{\underline{a}}+\eta^{\underline{a}}$ of the origin, $P_{\mathcal{S}}^{\underline{a}}$ is unchanged while $J_{\mathcal{S}}^{\underline{a}} \underline{b} \mapsto J_{\mathcal{S}}^{\underline{a}} \underline{b}+2 \eta^{[\underline{a}} P_{\mathcal{S}}^{\underline{b}}$. Thus $P_{\mathcal{S}}^{\underline{a}}$ and $J_{\mathcal{S}}^{\underline{a}} \underline{b}$ may be interpreted as the quasi-local energy-momentum and angular momentum of the matter fields associated with the spacelike 2 -surface $\mathcal{S}$, or, equivalently, to $D(\Sigma)$. Then the quasi-local mass and Pauli-Lubanski spin are defined, respectively, by the usual formulae $m_{\mathcal{S}}^{2}:=\eta_{\underline{a}} \underline{b} P_{\mathcal{S}}^{\underline{a}} P_{\mathcal{S}}^{\underline{b}}$ and $S_{\mathcal{S}}^{\underline{a}}:=\frac{1}{2} \varepsilon^{\underline{a}}{ }_{\underline{b}} \underline{c} \underline{d} P_{\mathcal{S}} J_{\mathcal{S}}^{\underline{c}} \underline{d}$. (If $m^{2} \neq 0$, then the dimensionally correct definition of the Pauli-Lubanski spin is $\frac{1}{m} S \frac{a}{\mathcal{S}}$.) As a consequence of the definitions $\eta_{\underline{a}} \underline{b} P_{\mathcal{S}}^{\underline{a}} S_{\mathcal{S}}^{\underline{b}}=0$ holds, i.e. if $P_{\mathcal{S}}^{\underline{a}}$ is timelike then $S_{\mathcal{S}}$ is spacelike or zero, but if $P_{\mathcal{S}}^{\underline{a}}$ is null (i.e. $\left.m_{\mathcal{S}}^{2}=0\right)$ then $S_{\mathcal{S}}$ is spacelike or proportional to $P_{\mathcal{S}}^{\underline{a}}$.

Obviously, we can form the flux integral of the current $T^{a b} \xi_{b}$ on the hypersurface even if $\xi^{a}$ is not a Killing vector, even in general curved spacetime:

$$
E_{\Sigma}\left[\xi^{a}\right]:=\int_{\Sigma} \xi_{e} T^{e f} \frac{1}{3 !} \varepsilon_{f a b c}
$$

Then, however, the integral $E_{\Sigma}\left[\xi^{a}\right]$ does depend on the hypersurface, because this is not connected with the spacetime symmetries. In particular, the vector field $\xi^{a}$ can be chosen to be the unit timelike normal $t^{a}$ of $\Sigma$. Since the component $\mu:=T_{a b} t^{a} t^{b}$ of the energy-momentum tensor is interpreted as the energy-density of the matter fields seen by the local observer $t^{a}$, it would be legitimate to interpret the corresponding integral $E_{\Sigma}\left[t^{a}\right]$ as 'the quasi-local energy of the matter fields seen by the fleet of observers being at rest with respect to $\Sigma$ '. Thus $E_{\Sigma}\left[t^{a}\right]$ defines a different concept of the quasi-local energy: While that based on $Q_{\mathcal{S}}[\mathbf{K}]$ is linked to some absolute element, namely to the translational Killing symmetries of the spacetime and the constant timelike vector fields can be interpreted as the observers 'measuring' this energy, $E_{\Sigma}\left[t^{a}\right]$ is completely independent of any absolute element of the spacetime and is based exclusively on the arbitrarily chosen fleet of observers. Thus, while $P_{\mathcal{S}}^{\underline{a}}$ is independent of the actual normal $t^{a}$ of $\mathcal{S}, E_{\Sigma}\left[\xi^{a}\right]$ (for non-Killing $\xi^{a}$ ) depends on $t^{a}$ intrinsically and is a genuine 3-hypersurface rather than a 2-surface integral.

If $P_{b}^{a}:=\delta_{b}^{a}-t^{a} t_{b}$, the orthogonal projection to $\Sigma$, then the part $j^{a}:=P_{b}^{a} T^{b c} t_{c}$ of the energymomentum tensor is interpreted as the momentum density seen by the observer $t^{a}$. Hence

$$
\left(t_{a} T^{a b}\right)\left(t_{c} T^{c d}\right) g_{b d}=\mu^{2}+h_{a b} j^{a} j^{b}=\mu^{2}-\left|j^{a}\right|^{2}
$$

is the square of the mass density of the matter fields, where $h_{a b}$ is the spatial metric in the plane orthogonal to $t^{a}$. If $T^{a b}$ satisfies the dominant energy condition (i.e. $T^{a b} V_{b}$ is a future directed non-spacelike vector for any future directed non-spacelike vector $V^{a}$, see for example [175]), then this is non-negative, and hence

$$
M_{\Sigma}:=\int_{\Sigma} \sqrt{\mu^{2}-\left|j^{e}\right|^{2}} \frac{1}{3 !} t^{f} \varepsilon_{f a b c}
$$

can also be interpreted as the quasi-local mass of the matter fields seen by the fleet of observers being at rest with respect to $\Sigma$, even in general curved spacetime. However, although in Minkowski

Living Reviews in Relativity

http: //www. livingreviews.org/lrr-2004-4 
spacetime $E_{\Sigma}[\mathbf{K}]$ for the four translational Killing vectors gives the four components of the energymomentum $P_{\mathcal{S}}^{\underline{a}}$, the mass $M_{\Sigma}$ is different from $m_{\mathcal{S}}$. In fact, while $m_{\mathcal{S}}$ is defined as the Lorentzian norm of $P_{\mathcal{S}}^{\underline{a}}$ with respect to the metric on the space of the translations, in the definition of $M_{\Sigma}$ first the norm of the current $T^{a b} t_{b}$ is taken with respect to the pointwise physical metric of the spacetime, and then its integral is taken. Nevertheless, because of the more advantageous properties (see Section 2.2.3 below), we prefer to represent the quasi-local energy(-momentum and angular momentum) of the matter fields in the form $Q_{\mathcal{S}}[\mathbf{K}]$ instead of $E_{\Sigma}\left[\xi^{a}\right]$.

Thus even if there is a gauge invariant and unambiguously defined energy-momentum density of the matter fields, it is not a priori clear how the various quasi-local quantities should be introduced. We will see in the second part of the present review that there are specific suggestions for the gravitational quasi-local energy that are analogous to $P_{\mathcal{S}}^{0}$, others to $E_{\Sigma}\left[t^{a}\right]$ and some to $M_{\Sigma}$.

\subsubsection{Hamiltonian introduction of the quasi-local quantities}

In the standard Hamiltonian formulation of the dynamics of the classical matter fields on a given (not necessarily flat) spacetime (see for example $[212,396]$ and references therein) the configuration and momentum variables, $\phi^{A}$ and $\pi_{A}$, respectively, are fields on a connected 3-manifold $\Sigma$, which is interpreted as the typical leaf of a foliation $\Sigma_{t}$ of the spacetime. The foliation can be characterized on $\Sigma$ by a function $N$, called the lapse. The evolution of the states in the spacetime is described with respect to a vector field $K^{a}=N t^{a}+N^{a}$ ('evolution vector field' or 'general time axis'), where $t^{a}$ is the future directed unit normal to the leaves of the foliation and $N^{a}$ is some vector field, called the shift, being tangent to the leaves. If the matter fields have gauge freedom, then the dynamics of the system is constrained: Physical states can be only those that are on the constraint surface, specified by the vanishing of certain functions $C_{\mathbf{i}}=C_{\mathbf{i}}\left(\phi^{A}, D_{e} \phi^{A}, \ldots, \pi_{A}, D_{e} \pi_{A}, \ldots\right), \mathbf{i}=1, \ldots, n$, of the canonical variables and their derivatives up to some finite order, where $D_{e}$ is the covariant derivative operator in $\Sigma$. Then the time evolution of the states in the phase space is governed by the Hamiltonian, which has the form

$$
H[\mathbf{K}]=\int_{\Sigma}\left(\mu N+j_{a} N^{a}+C_{\mathbf{i}} N^{\mathbf{i}}+D_{a} Z^{a}\right) d \Sigma .
$$

Here $d \Sigma$ is the induced volume element, the coefficients $\mu$ and $j_{a}$ are local functions of the canonical variables and their derivatives up to some finite order, the $N^{\mathrm{i}}$,s are functions on $\Sigma$, and $Z^{a}$ is a local function of the canonical variables, the lapse, the shift, the functions $N^{\mathrm{i}}$, and their derivatives up to some finite order. The part $C_{\mathbf{i}} N^{\mathbf{i}}$ of the Hamiltonian generates gauge motions in the phase space, and the functions $N^{\mathbf{i}}$ are interpreted as the freely specifiable 'gauge generators'.

However, if we want to recover the field equations for $\phi^{A}$ (which are partial differential equations on the spacetime with smooth coefficients for the smooth field $\phi^{A}$ ) on the phase space as the Hamilton equations and not some of their distributional generalizations, then the functional differentiability of $H[\mathbf{K}]$ must be required in the strong sense of [387 $]^{1}$. Nevertheless, the functional differentiability (and, in the asymptotically flat case, also the existence) of $H[\mathbf{K}]$ requires some boundary conditions on the field variables, and may yield restrictions on the form of $Z^{a}$. It may happen that for a given $Z^{a}$ only too restrictive boundary conditions would be able to ensure the functional differentiability of the Hamiltonian, and hence the 'quasi-local phase space' defined with these boundary conditions would contain only very few (or no) solutions of the field equations. In this case $Z^{a}$ should be modified. In fact, the boundary conditions are connected to the nature of the physical situations considered. For example, in electrodynamics different boundary conditions must be imposed if the boundary is to represent a conducting or an insulating surface.

\footnotetext{
${ }^{1}$ Sometimes in the literature this requirement is introduced as some new principle in the Hamiltonian formulation of the fields, but its real content is not more than to ensure that the Hamilton equations coincide with the field equations.
} 
Unfortunately, no universal principle or 'canonical' way of finding the 'correct' boundary term and the boundary conditions is known.

In the asymptotically flat case the value of the Hamiltonian on the constraint surface defines the total energy-momentum and angular momentum, depending on the nature of $K^{a}$, in which the total divergence $D_{a} Z^{a}$ corresponds to the ambiguity of the superpotential 2-form $\cup[\mathbf{K}]_{a b}$ : An identically conserved quantity can always be added to the Hamiltonian (provided its functional differentiability is preserved). The energy density and the momentum density of the matter fields can be recovered as the functional derivative of $H[\mathbf{K}]$ with respect to the lapse $N$ and the shift $N^{a}$, respectively. In principle, the whole analysis can be repeated quasi-locally too. However, apart from the promising achievements of $[7,8,327]$ for the Klein-Gordon, Maxwell, and the YangMills-Higgs fields, as far as we know, such a systematic quasi-local Hamiltonian analysis of the matter fields is still lacking.

\subsubsection{Properties of the quasi-local quantities}

Suppose that the matter fields satisfy the dominant energy condition. Then $E_{\Sigma}\left[\xi^{a}\right]$ is also nonnegative for any non-spacelike $\xi^{a}$, and, obviously, $E_{\Sigma}\left[t^{a}\right]$ is zero precisely when $T^{a b}=0$ on $\Sigma$, and hence, by the conservation laws (see for example Page 94 of [175]), on the whole domain of dependence $D(\Sigma)$. Obviously, $M_{\Sigma}=0$ if and only if $L^{a}:=T^{a b} t_{b}$ is null on $\Sigma$. Then by the dominant energy condition it is a future pointing vector field on $\Sigma$, and $L_{a} T^{a b}=0$ holds. Therefore, $T^{a b}$ on $\Sigma$ has a null eigenvector with zero eigenvalue, i.e. its algebraic type on $\Sigma$ is pure radiation.

The properties of the quasi-local quantities based on $Q_{\mathcal{S}}[\mathbf{K}]$ in Minkowski spacetime are, however, more interesting. Namely, assuming that the dominant energy condition is satisfied, one can prove $[354,358]$ that

1. $P_{\mathcal{S}}^{\underline{a}}$ is a future directed nonspacelike vector, $m_{\mathcal{S}}^{2} \geq 0$;

2. $P_{\mathcal{S}}^{\underline{a}}=0$ if and only if $T_{a b}=0$ on $D(\Sigma)$;

3. $m_{\mathcal{S}}^{2}=0$ if and only if the algebraic type of the matter on $D(\Sigma)$ is pure radiation, i.e. $T_{a b} L^{b}=0$ holds for some constant null vector $L^{a}$. Then $T_{a b}=\tau L_{a} L_{b}$ for some non-negative function $\tau$, whenever $P_{\mathcal{S}}^{\underline{a}}=e L^{\underline{a}}$, where $L^{\underline{a}}:=L^{a} \vartheta^{\frac{a}{a}}$ and $e:=\int_{\Sigma} \tau L^{a} \frac{1}{3 !} \varepsilon_{a b c d}$;

4. For $m_{\mathcal{S}}^{2}=0$ the angular momentum has the form $J_{\mathcal{S}}^{\underline{a}} \underline{\underline{b}}=e^{\underline{\underline{a}}} L^{\underline{\underline{b}}}-e^{\underline{\underline{b}}} L^{\underline{a}}$, where $e^{\underline{a}}:=$ $\int_{\Sigma} x^{\underline{a}} \tau L^{a} \frac{1}{3 !} \varepsilon_{a b c d}$. Thus, in particular, the Pauli-Lubanski spin is zero.

Therefore, the vanishing of the quasi-local energy-momentum characterizes the 'vacuum state' of the classical matter fields completely, and the vanishing of the quasi-local mass is equivalent to special configurations representing pure radiation.

Since $E_{\Sigma}\left[t^{a}\right]$ and $M_{\Sigma}$ are integrals of functions on a hypersurface, they are obviously additive, i.e. for example for any two hypersurfaces $\Sigma_{1}$ and $\Sigma_{2}$ (having common points at most on their boundaries $\mathcal{S}_{1}$ and $\mathcal{S}_{2}$ ) one has $E_{\Sigma_{1} \cup \Sigma_{2}}\left[t^{a}\right]=E_{\Sigma_{1}}\left[t^{a}\right]+E_{\Sigma_{2}}\left[t^{a}\right]$. On the other hand, the additivity of $P_{\mathcal{S}}^{\underline{a}}$ is a slightly more delicate problem. Namely, $P_{\mathcal{S}_{1}}^{a}$ and $P_{\mathcal{S}_{2}}^{\underline{a}}$ are elements of the dual space of the translations, and hence we can add them and, as in the previous case, we obtain additivity. However, this additivity comes from the absolute parallelism of the Minkowski spacetime: The quasi-local energy-momenta of the different 2-surfaces belong to one and the same vector space. If there were no natural connection between the Killing vectors on different 2-surfaces, then the energy-momenta would belong to different vector spaces, and they could not be added. We will see that the quasi-local quantities discussed in Sections 7, 8, and 9 belong to vector spaces dual to their own 'quasi-Killing vectors', and there is no natural way of adding the energy-momenta of different surfaces.

Living Reviews in Relativity

http://www. livingreviews.org/lrr-2004-4 


\subsubsection{Global energy-momenta and angular momenta}

If $\Sigma$ extends either to spatial or future null infinity, then, as is well known, the existence of the limit of the quasi-local energy-momentum can be ensured by slightly faster than $\mathcal{O}\left(r^{-3}\right)$ (for example by $\mathcal{O}\left(r^{-4}\right)$ ) fall-off of the energy-momentum tensor, where $r$ is any spatial radial distance. However, the finiteness of the angular momentum and centre-of-mass is not ensured by the $\mathcal{O}\left(r^{-4}\right)$ fall-off. Since the typical fall-off of $T_{a b}$ - for example for the electromagnetic field - is $\mathcal{O}\left(r^{-4}\right)$, we may not impose faster than this, because otherwise we would exclude the electromagnetic field from our investigations. Thus, in addition to the $\mathcal{O}\left(r^{-4}\right)$ fall-off, six global integral conditions for the leading terms of $T_{a b}$ must be imposed. At the spatial infinity these integral conditions can be ensured by explicit parity conditions, and one can show that the 'conservation equations' $T^{a b}{ }_{; b}=0$ (as evolution equations for the energy density and momentum density) preserve these fall-off and parity conditions [364].

Although quasi-locally the vanishing of the mass does not imply the vanishing of the matter fields themselves (the matter fields must be pure radiative field configurations with plane wave fronts), the vanishing of the total mass alone does imply the vanishing of the fields. In fact, by the vanishing of the mass the fields must be plane waves, furthermore by $T_{a b}=\mathcal{O}\left(r^{-4}\right)$ they must be asymptotically vanishing at the same time. However, a plane wave configuration can be asymptotically vanishing only if it is vanishing.

\subsubsection{Quasi-local radiative modes and a classical version of the holography for matter fields}

By the results of the previous Section 2.2.4 the vanishing of the quasi-local mass, associated with a closed spacelike 2-surface $\mathcal{S}$, implies that the matter must be pure radiation on a 4-dimensional globally hyperbolic domain $D(\Sigma)$. Thus $m_{\mathcal{S}}=0$ characterizes 'simple', 'elementary' states of the matter fields. In the present section we review how these states on $D(\Sigma)$ can be characterized completely by data on the 2 -surface $\mathcal{S}$, and how these states can be used to formulate a classical version of the holographic principle.

For the (real or complex) linear massless scalar field $\phi$ and the Yang-Mills fields, represented by the symmetric spinor fields $\phi_{A B}^{\alpha}, \alpha=1, \ldots, N$, where $N$ is the dimension of the gauge group, the vanishing of the quasi-local mass is equivalent [365] to plane waves and the $p p$-wave solutions of Coleman [118], respectively. Then the condition $T_{a b} L^{b}=0$ implies that these fields are completely determined on the whole $D(\Sigma)$ by their value on $\mathcal{S}$ (whenever the spinor fields $\phi_{A B}^{\alpha}$ are necessarily null: $\phi_{A B}^{\alpha}=\phi^{\alpha} O_{A} O_{B}$, where $\phi^{\alpha}$ are complex functions and $O_{A}$ is a constant spinor field such that $\left.L_{a}=O_{A} \bar{O}_{A^{\prime}}\right)$. Similarly, the null linear zero-rest-mass fields $\phi_{A B \ldots E}=\phi O_{A} O_{B} \ldots O_{E}$ on $D(\Sigma)$ with any spin and constant spinor $O_{A}$ are completely determined by their value on $\mathcal{S}$. Technically, these results are based on the unique complex analytic structure of the $u=$ const. 2-surfaces foliating $\Sigma$, where $L_{a}=\nabla_{a} u$, and by the field equations the complex functions $\phi$ and $\phi^{\alpha}$ turn out to be anti-holomorphic [358]. Assuming, for the sake of simplicity, that $\mathcal{S}$ is future and past convex in the sense of Section 4.1.3 below, the independent boundary data for such a pure radiative solution consist of a constant spinor field on $\mathcal{S}$ and a real function with one and another with two variables. Therefore, the pure radiative modes on $D(\Sigma)$ can be characterized completely by appropriate data (the so-called holographic data) on the 'screen' $\mathcal{S}$.

These 'quasi-local radiative modes' can be used to map any continuous spinor field on $D(\Sigma)$ to a collection of holographic data. Indeed, the special radiative solutions of the form $\phi O^{A}$ (with fixed constant spinor field $O^{A}$ ) together with their complex conjugate define a dense subspace in the space of all continuous spinor fields on $\Sigma$. Thus every such spinor field can be expanded by the special radiative solutions, and hence can also be represented by the corresponding family of holographic data. Therefore, if we fix a foliation of $D(\Sigma)$ by spacelike Cauchy surfaces $\Sigma_{t}$, then every spinor field on $D(\Sigma)$ can also be represented on $\mathcal{S}$ by a time dependent family of holographic 
data, too [365]. This fact may be a specific manifestation in the classical non-gravitational physics of the holographic principle (see Section 13.4.2).

Living Reviews in Relativity

http://www.livingreviews . org/lrr-2004-4 


\section{On the Energy-Momentum and Angular Momentum of Gravitating Systems}

\subsection{On the gravitational energy-momentum and angular momentum density: The difficulties}

\subsubsection{The root of the difficulties}

The action $I_{\mathrm{m}}$ for the matter fields was a functional of both kinds of fields, thus one could take the variational derivatives both with respect to $\Phi_{N_{b}}^{a \ldots}$ and $g^{a b}$. The former gave the field equations, while the latter defined the symmetric energy-momentum tensor. Moreover, $g_{a b}$ provided a metrical geometric background, in particular a covariant derivative, for carrying out the analysis of the matter fields. The gravitational action $I_{\mathrm{g}}$ is, on the other hand, a functional of the metric alone, and its variational derivative with respect to $g^{a b}$ yields the gravitational field equations. The lack of any further geometric background for describing the dynamics of $g^{a b}$ can be traced back to the principle of equivalence [22], and introduces a huge gauge freedom in the dynamics of $g^{a b}$ because that should be formulated on a bare manifold: The physical spacetime is not simply a manifold $M$ endowed with a Lorentzian metric $g_{a b}$, but the isomorphism class of such pairs, where $\left(M, g_{a b}\right)$ and $\left(M, \phi^{*} g_{a b}\right)$ are considered to be equivalent for any diffeomorphism $\phi$ of $M$ onto itself ${ }^{2}$. Thus we do not have, even in principle, any gravitational analog of the symmetric energy-momentum tensor of the matter fields. In fact, by its very definition, $T_{a b}$ is the source-current for gravity, like the current $J_{\mathbf{A}}^{a}:=\delta I_{p} / \delta A_{a}^{\mathbf{A}}$ in Yang-Mills theories (defined by the variational derivative of the action functional of the particles, e.g. of the fermions, interacting with a Yang-Mills field $A_{a}^{\mathbf{A}}$ ), rather than energymomentum. The latter is represented by the Noether currents associated with special spacetime displacements. Thus, in spite of the intimate relation between $T_{a b}$ and the Noether currents, the proper interpretation of $T_{a b}$ is only the source density for gravity, and hence it is not the symmetric energy-momentum tensor whose gravitational counterpart must be searched for. In particular, the Bel-Robinson tensor $T_{a b c d}:=\psi_{A B C D} \bar{\psi}_{A^{\prime} B^{\prime} C^{\prime} D^{\prime}}$, given in terms of the Weyl spinor, (and its generalizations introduced by Senovilla [333, 332]), being a quadratic expression of the curvature (and its derivatives), is (are) expected to represent only 'higher order' gravitational energy-momentum. (Note that according to the original tensorial definition the Bel-Robinson tensor is one-fourth the expression above. Our convention follows that of Penrose and Rindler [312].) In fact, the physical dimension of the Bel-Robinson 'energy-density' $T_{a b c d} t^{a} t^{b} t^{c} t^{d}$ is $\mathrm{cm}^{-4}$, and hence (in the traditional units) there are no powers $A$ and $B$ such that $c^{A} G^{B} T_{a b c d} t^{a} t^{b} t^{c} t^{d}$ would have energy-density dimension. Here $c$ is the speed of light and $G$ is Newton's gravitational constant. As we will see, the Bel-Robinson 'energy-momentum density' $T_{a b c d} t^{b} t^{c} t^{d}$ appears naturally in connection with the quasi-local energy-momentum and spin-angular momentum expressions for small spheres only in higher order terms. Therefore, if we want to associate energy-momentum and angular momentum with the gravity itself in a Lagrangian framework, then it is the gravitational counterpart of the canonical energy-momentum and spin tensors and the canonical Noether current built from them that should be introduced. Hence it seems natural to apply the Lagrange-Belinfante-Rosenfeld procedure, sketched in the previous section, to gravity too [56, 57, 323, 193, 194, 352].

\footnotetext{
${ }^{2}$ Since we do not have a third kind of device to specify the spatio-temporal location of the devices measuring the spacetime geometry, we do not have any further operationally defined, maybe non-dynamical background, just in accordance with the principle of equivalence. If there were some non-dynamical background metric $g_{a b}^{0}$ on $M$, then by requiring $g_{a b}^{0}=\phi^{*} g_{a b}^{0}$ we could reduce the almost arbitrary diffeomorphism $\phi$ (essentially four arbitrary functions of four variables) to a transformation depending on at most ten parameters.
} 


\subsubsection{Pseudotensors}

The lack of any background geometric structure in the gravitational action yields, first, that any vector field $K^{a}$ generates a symmetry of the matter plus gravity system. Its second consequence is the need for an auxiliary derivative operator, e.g. the Levi-Civita covariant derivative coming from an auxiliary, non-dynamical background metric (see for example [231, 316]), or a background (usually torsion free, but not necessarily flat) connection (see for example [215]), or the partial derivative coming from a local coordinate system (see for example [382]). Though the natural expectation would be that the final results be independent of these background structures, as is well known, the results do depend on them.

In particular [352], for Hilbert's second order Lagrangian $L_{\mathrm{H}}:=R / 16 \pi G$ in a fixed local coordinate system $\left\{x^{\alpha}\right\}$ and derivative operator $\partial_{\mu}$ instead of $\nabla_{e}$, Equation (4) gives precisely Møller's energy-momentum pseudotensor ${ }_{\mathrm{M}} \theta^{\alpha}{ }_{\beta}$, which was defined originally through the superpotential equation $\sqrt{|g|}\left(8 \pi G_{\mathrm{M}} \theta^{\alpha}{ }_{\beta}-G^{\alpha}{ }_{\beta}\right)=\partial_{\mu \mathrm{M}} \cup_{\beta}{ }^{\alpha \mu}$, where ${ }_{\mathrm{M}} \cup_{\beta}{ }^{\alpha \mu}:=\sqrt{|g|} g^{\alpha \rho} g^{\mu \omega}\left(\partial_{[\omega} g_{\rho] \beta}\right)$ is the so-called Møller superpotential [270]. (For another simple and natural introduction of Møller's energy-momentum pseudotensor see [104].) For the spin pseudotensor Equation (2) gives

$$
8 \pi G \sqrt{|g|}_{\mathrm{M}} \sigma_{\beta}^{\mu \alpha}={ }_{\mathrm{M}} \cup_{\beta}{ }^{\alpha \mu}+\partial_{\nu}\left(\sqrt{|g|} \delta_{\beta}^{[\mu} g^{\nu] \alpha}\right)
$$

which is in fact only pseudotensorial. Similarly, the contravariant form of these pseudotensors and the corresponding canonical Noether current are also pseudotensorial. We saw in Section 2.1.2 that a specific combination of the canonical energy-momentum and spin tensors gave the symmetric energy-momentum tensor, which is gauge invariant even if the matter fields have gauge freedom, and one might hope that the analogous combination of the energy-momentum and spin pseudotensors gives a reasonable tensorial energy-momentum density for the gravitational field. The analogous expression is, in fact, tensorial, but unfortunately it is just minus the Einstein tensor $[352,353]^{3}$. Therefore, to use the pseudotensors a 'natural' choice for a 'preferred' coordinate system would be needed. This could be interpreted as a gauge choice, or reference configuration.

A further difficulty is that the different pseudotensors may have different (potential) significance. For example, for any fixed $k \in \mathbb{R}$ Goldberg's $2 k$-th symmetric pseudotensor $t_{(2 k)}^{\alpha \beta}$ is defined by $2|g|^{k+1}\left(8 \pi G t_{(2 k)}^{\alpha \beta}-G^{\alpha \beta}\right):=\partial_{\mu} \partial_{\nu}\left[|g|^{k+1}\left(g^{\alpha \beta} g^{\mu \nu}-g^{\alpha \nu} g^{\beta \mu}\right)\right]$ (which, for $k=0$, reduces to the Landau-Lifshitz pseudotensor, the only symmetric pseudotensor which is a quadratic expression of the first derivatives of the metric) [162]. However, by Einstein's equations this definition implies that $\partial_{\alpha}\left[|g|^{k+1}\left(t_{(2 k)}^{\alpha \beta}+T^{\alpha \beta}\right)\right]=0$. Hence what is (coordinate-)divergence-free (i.e. 'pseudoconserved') cannot be interpreted as the sum of the gravitational and matter energy-momentum densities. Indeed, the latter is $|g|^{1 / 2} T^{\alpha \beta}$, while the second term in the divergence equation has an extra weight $|g|^{k+1 / 2}$. Thus there is only one pseudotensor in this series, $t_{(-1)}^{\alpha \beta}$, which satisfies the 'conservation law' with the correct weight. In particular, the Landau-Lifshitz pseudotensor $t_{(0)}^{\alpha \beta}$ also has this defect. On the other hand, the pseudotensors coming from some action (the 'canonical pseudotensors') appear to be free of this kind of difficulties (see also [352, 353]). Classical excellent reviews on these (and several other) pseudotensors are [382, 59, 9, 163], and for some recent ones (using background geometric structures) see for example [137, 138, 79, 154, 155, 228, 316]. We return to the discussion of pseudotensors in Sections 3.3.1 and 11.3.4.

\footnotetext{
${ }^{3}$ Since Einstein's Lagrangian is only weakly diffeomorphism invariant, the situation would even be worse if we used Einstein's Lagrangian. The corresponding canonical quantities would still be coordinate dependent, though in certain 'natural' coordinate system they yield reasonable results (see for example [2] and references therein).
}

Living Reviews in Relativity

http://www. livingreviews.org//rr-2004-4 


\subsubsection{Strategies to avoid pseudotensors I: Background metrics/connections}

One way of avoiding the use of the pseudotensorial quantities is to introduce an explicit background connection [215] or background metric [322, 229, 233, 231, 230, 315, 135]. (The superpotential of Katz, Bičák, and Lyndel-Bell [230] has been rediscovered recently by Chen and Nester [108] in a completely different way. We return to the discussion of the latter in Section 11.3.2.) The advantage of this approach would be that we could use the background not only to derive the canonical energymomentum and spin tensors, but to define the vector fields $K^{a}$ as the symmetry generators of the background. Then the resulting Noether currents are without doubt tensorial. However, they depend explicitly on the choice of the background connection or metric not only through $K^{a}$ : The canonical energy-momentum and spin tensors themselves are explicitly background-dependent. Thus, again, the resulting expressions would have to be supplemented by a 'natural' choice for the background, and the main question is how to find such a 'natural' reference configuration from the infinitely many possibilities.

\subsubsection{Strategies to avoid pseudotensors II: The tetrad formalism}

In the tetrad formulation of general relativity the $g_{a b}$-orthonormal frame fields $\left\{E_{a}^{a}\right\}, \underline{a}=0, \ldots, 3$, are chosen to be the gravitational field variables [386, 236]. Re-expressing the Hilbert Lagrangian (i.e. the curvature scalar) in terms of the tetrad field and its partial derivatives in some local coordinate system, one can calculate the canonical energy-momentum and spin by Equations (4) and (2), respectively. Not surprisingly at all, we recover the pseudotensorial quantities that we obtained in the metric formulation above. However, as realized by Møller [271], the use of the tetrad fields as the field variables instead of the metric makes it possible to introduce a first order, scalar Lagrangian for Einstein's field equations: If $\gamma_{\underline{e}}^{\underline{a}}:=E_{\underline{e}}^{e} \gamma_{e \underline{b}}^{\underline{a}}:=E_{\underline{e}}^{e} \vartheta_{a}^{\underline{a}} \nabla_{e} E_{\underline{b}}^{a}$, the Ricci rotation coefficients, then Møller's tetrad Lagrangian is

$$
L:=\frac{1}{16 \pi G}\left[R-2 \nabla_{a}\left(E_{\underline{a}}^{a} \eta^{\underline{a} \underline{b}} \gamma_{\underline{\underline{c}} \underline{b}}^{\underline{b}}\right)\right]=\frac{1}{16 \pi G}\left(E_{\underline{a}}^{a} E_{\underline{b}}^{b}-E_{\underline{a}}^{b} E_{\underline{b}}^{a}\right) \gamma_{\underline{a} \underline{a}}^{\underline{a}} \gamma_{\underline{b}}^{\underline{c} \underline{b}} .
$$

(Here $\left\{\vartheta_{a}^{a}\right\}$ is the 1 -form basis dual to $\left\{E_{\underline{a}}^{a}\right\}$.) Although $L$ depends on the actual tetrad field $\left\{E_{a}^{a}\right\}$, it is weakly $O(1,3)$-invariant. Møller's Lagrangian has a nice uniqueness property [299]: Any first order scalar Lagrangian built from the tetrad fields, whose Euler-Lagrange equations are the Einstein equations, is Møller's Lagrangian. Using Dirac spinor variables Nester and Tung found a first order spinor Lagrangian [288], which turned out to be equivalent to Møller's Lagrangian [383]. Another first order spinor Lagrangian, based on the use of the two-component spinors and the anti-self-dual connection, was suggested by Tung and Jacobson [384]. Both Lagrangians yield a well-defined Hamiltonian, reproducing the standard ADM energy-momentum in asymptotically flat spacetimes. The canonical energy-momentum $\theta^{\alpha}{ }_{\beta}$ derived from Equation (9) using the components of the tetrad fields in some coordinate system as the field variables is still pseudotensorial, but, as Møller realized, it has a tensorial superpotential:

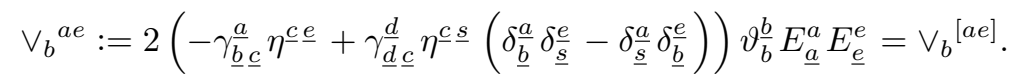

The canonical spin turns out to be essentially $\vee_{b}{ }^{a e}$, i.e. a tensor. The tensorial nature of the superpotential makes it possible to introduce a canonical energy-momentum tensor for the gravitational 'field'. Then the corresponding canonical Noether current $C^{a}[\mathbf{K}]$ will also be tensorial and satisfies

$$
8 \pi G C^{a}[\mathbf{K}]=G^{a b} K_{b}+\frac{1}{2} \nabla_{c}\left(K^{b} \vee_{b}^{a c}\right) .
$$

Therefore, the canonical Noether current derived from Møller's tetrad Lagrangian is independent of the background structure (i.e. the coordinate system) that we used to do the calculations (see 
also [352]). However, $C^{a}[\mathbf{K}]$ depends on the actual tetrad field, and hence a preferred class of frame fields, i.e. an $O(1,3)$-gauge reduction, is needed. Thus the explicit background-dependence of the final result of other approaches has been transformed into an internal $O(1,3)$-gauge dependence. It is important to realize that this difficulty always appears in connection with the gravitational energy-momentum and angular momentum, at least in disguise. In particular, the Hamiltonian approach in itself does not yield well-defined energy-momentum density for the gravitational 'field' (see for example [282, 263]). Thus in the tetrad approach the canonical Noether current should be supplemented by a gauge condition for the tetrad field. Such a gauge condition could be some spacetime version of Nester's gauge conditions (in the form of certain partial differential equations) for the orthonormal frames of Riemannian manifolds [281, 284]. Furthermore, since $C^{a}[\mathbf{K}]+T^{a b} K_{b}$ is conserved for any vector field $K^{a}$, in the absence of the familiar Killing symmetries of the Minkowski spacetime it is not trivial to define the 'translations' and 'rotations', and hence the energy-momentum and angular momentum. To make them well-defined additional ideas would be needed.

\subsubsection{Strategies to avoid pseudotensors III: Higher derivative currents}

Giving up the paradigm that the Noether current should depend only on the vector field $K^{a}$ and its first derivative - i.e. if we allow a term $\dot{B}^{a}$ to be present in the Noether current (3) even if the Lagrangian is diffeomorphism invariant - one naturally arrives at Komar's tensorial superpotential ${ }_{\mathrm{K}} \vee[\mathbf{K}]^{a b}:=\nabla^{[a} K^{b]}$ and the corresponding Noether current [242] (see also [59]). Although its independence of any background structure (viz. its tensorial nature) and uniqueness property (see Komar [242] quoting Sachs) is especially attractive, the vector field $K^{a}$ is still to be determined.

\subsection{On the global energy-momentum and angular momentum of gravi- tating systems: The successes}

As is well known, in spite of the difficulties with the notion of the gravitational energy-momentum density discussed above, reasonable total energy-momentum and angular momentum can be associated with the whole spacetime provided it is asymptotically flat. In the present section we recall the various forms of them. As we will see, most of the quasi-local constructions are simply 'quasi-localizations' of the total quantities. Obviously, the technique used in the 'quasi-localization' does depend on the actual form of the total quantities, yielding mathematically inequivalent definitions for the quasi-local quantities. We return to the discussion of the tools needed in the quasi-localization procedures in Sections 4.2 and 4.3. Classical, excellent reviews of global energymomentum and angular momentum are [151, 163, 15, 289, 394, 313], and a recent review of conformal infinity (with special emphasis on its applicability in numerical relativity) is [144]. Reviews of the positive energy proofs from the first third of the eighties are [202, 314].

\subsubsection{Spatial infinity: Energy-momentum}

There are several mathematically inequivalent definitions of asymptotic flatness at spatial infinity $[151,344,23,48,148]$. The traditional definition is based on the existence of a certain asymptotically flat spacelike hypersurface. Here we adopt this definition, which is probably the weakest one in the sense that the spacetimes that are asymptotically flat in the sense of any reasonable definition are asymptotically flat in the traditional sense too. A spacelike hypersurface $\Sigma$ will be called $k$-asymptotically flat if for some compact set $K \subset \Sigma$ the complement $\Sigma-K$ is diffeomorphic to $\mathbb{R}^{3}$ minus a solid ball, and there exists a (negative definite) metric ${ }_{0} h_{a b}$ on $\Sigma$, which is flat on $\Sigma-K$, such that the components of the difference of the physical and the background metrics, $h_{\mathbf{i j}}-{ }_{0} h_{\mathbf{i} \mathbf{j}}$, and of the extrinsic curvature $\chi_{\mathbf{i j}}$ in the ${ }_{0} h_{\mathbf{i j}}$-Cartesian coordinate system $\left\{x^{\mathbf{k}}\right\}$ fall off

Living Reviews in Relativity

http://www. livingreviews . org//rr-2004-4 
as $r^{-k}$ and $r^{-k-1}$, respectively, for some $k>0$ and $r^{2}:=\delta_{\mathbf{i j}} x^{\mathbf{i}} x^{\mathbf{j}}[319,47]$. These conditions make it possible to introduce the notion of asymptotic spacetime Killing vectors, and to speak about asymptotic translations and asymptotic boost rotations. $\Sigma-K$ together with the metric and extrinsic curvature is called the asymptotic end of $\Sigma$. In a more general definition of asymptotic flatness $\Sigma$ is allowed to have finitely many such ends.

As is well known, finite and well-defined ADM energy-momentum $[11,13,12,14]$ can be associated with any $k$-asymptotically flat spacelike hypersurface if $k>\frac{1}{2}$ by taking the value on the constraint surface of the Hamiltonian $H\left[K^{a}\right]$, given for example in $[319,47]$, with the asymptotic translations $K^{a}$ (see [112, 37, 291, 113]). In its standard form this is the $r \rightarrow \infty$ limit of a 2-surface integral of the first derivatives of the induced 3-metric $h_{a b}$ and of the extrinsic curvature $\chi_{a b}$ for spheres of large coordinate radius $r$. The ADM energy-momentum is an element of the space dual to the space of the asymptotic translations, and transforms as a Lorentzian 4-vector with respect to asymptotic Lorentz transformations of the asymptotic Cartesian coordinates.

The traditional ADM approach to the introduction of the conserved quantities and the Hamiltonian analysis of general relativity is based on the $3+1$ decomposition of the fields and the spacetime. Thus it is not a priori clear that the energy and spatial momentum form a Lorentz vector (and the spatial angular momentum and centre-of-mass, discussed below, form an antisymmetric tensor). One had to check a posteriori that the conserved quantities obtained in the $3+1$ form are, in fact, Lorentz-covariant. To obtain manifestly Lorentz-covariant quantities one should not do the $3+1$ decomposition. Such a manifestly Lorentz-covariant Hamiltonian analysis was suggested first by Nester [280], and he was able to recover the ADM energy-momentum in a natural way (see also Section 11.3 below).

Another form of the ADM energy-momentum is based on Møller's tetrad superpotential [163]: Taking the flux integral of the current $C^{a}[\mathbf{K}]+T^{a b} K_{b}$ on the spacelike hypersurface $\Sigma$, by Equation (11) the flux can be rewritten as the $r \rightarrow \infty$ limit of the 2-surface integral of Møller's superpotential on spheres of large $r$ with the asymptotic translations $K^{a}$. Choosing the tetrad field $E_{a}^{a}$ to be adapted to the spacelike hypersurface and assuming that the frame $E_{\underline{a}}^{a}$ tends to a constant Cartesian one as $r^{-k}$, the integral reproduces the ADM energy-momentum. The same expression can be obtained by following the familiar Hamiltonian analysis using the tetrad variables too: By the standard scenario one can construct the basic Hamiltonian [282]. This Hamiltonian, evaluated on the constraints, turns out to be precisely the flux integral of $C^{a}[\mathbf{K}]+T^{a b} K_{b}$ on $\Sigma$.

A particularly interesting and useful expression for the ADM energy-momentum is possible if the tetrad field is considered to be a frame field built from a normalized spinor dyad $\left\{\lambda_{A}^{A}\right\}$, $\underline{A}=0,1$, on $\Sigma$ which is asymptotically constant (see Section 4.2 .3 below). (Thus underlined capital Roman indices are concrete name spinor indices.) Then, for the components of the ADM energy-momentum in the constant spinor basis at infinity, Møller's expression yields the limit of

$$
P^{\underline{A} \underline{B}^{\prime}}=\frac{1}{4 \pi G} \oint_{\mathcal{S}} \frac{\mathrm{i}}{2}\left(\bar{\lambda} \bar{B}^{\prime}{ }^{\prime} \nabla_{B B^{\prime}} \lambda \frac{A}{A}-\bar{\lambda} \bar{B}^{\prime}{ }^{\prime} \nabla_{A A^{\prime}} \lambda \frac{A}{B}\right)
$$

as the 2-surface $\mathcal{S}$ is blown up to approach infinity. In fact, to recover the ADM energy-momentum in the form (12), the spinor fields $\lambda_{A}^{A}$ need not be required to form a normalized spinor dyad, it is enough that they form an asymptotically constant normalized dyad, and we have to use the fact that the generator vector field $K^{a}$ has asymptotically constant components $K^{\underline{A}} \underline{A}^{\prime}$ in the asymptotically constant frame field $\lambda_{\underline{A}}^{A} \bar{\lambda}_{\underline{A^{\prime}}}^{A^{\prime}}$. Thus $K^{a}=K \underline{A} \underline{A}^{\prime} \lambda_{\underline{A}}^{A} \bar{\lambda}_{\underline{A^{\prime}}}^{A^{\prime}}$ can be interpreted as an asymptotic translation. The complex-valued 2-form in the integrand of Equation (12) will be denoted by $u\left(\lambda \underline{A}, \bar{\lambda}^{\underline{B}}\right)_{a b}$, and is called the Nester-Witten 2-form. This is 'essentially Hermitian' and connected with Komar's superpotential: For any two spinor fields $\alpha^{A}$ and $\beta^{A}$ one has

$$
\begin{aligned}
& u(\alpha, \bar{\beta})_{a b}-\overline{u(\beta, \bar{\alpha})_{a b}}=-\mathrm{i} \nabla_{[a} X_{b]}, \\
& u(\alpha, \bar{\beta})_{a b}+\overline{u(\beta, \bar{\alpha})_{a b}}=\frac{1}{2} \nabla_{c} X_{d} \varepsilon^{c d}{ }_{a b}+\mathrm{i}\left(\varepsilon_{A^{\prime} B^{\prime}} \alpha_{(A} \nabla_{B) C^{\prime}} \bar{\beta}^{C^{\prime}}-\varepsilon_{A B} \bar{\beta}_{\left(A^{\prime}\right.} \nabla_{\left.B^{\prime}\right) C} \alpha^{C}\right),
\end{aligned}
$$


where $X_{a}:=\alpha_{A} \bar{\beta}_{A^{\prime}}$ and overline denotes complex conjugation. Thus, apart from the terms in Equation (14) involving $\nabla_{A^{\prime} A} \alpha^{A}$ and $\nabla_{A A^{\prime}} \bar{\beta}^{A^{\prime}}$, the Nester-Witten 2-form $u(\alpha, \bar{\beta})_{a b}$ is just $-\frac{\mathrm{i}}{2}\left(\nabla_{[a} X_{b]}+\right.$ $i \nabla_{[c} X_{d]} \frac{1}{2} \varepsilon^{c d}{ }_{a b}$ ), i.e. the anti-self-dual part of the curl of $-\frac{i}{2} X_{a}$. (The original expressions by Witten and Nester were given using Dirac rather than two-component Weyl spinors [397, 279]. The 2 -form $u(\alpha, \bar{\beta})_{a b}$ in the present form using the two-component spinors appeared first probably in [205].) Although many interesting and original proofs of the positivity of the ADM energy are known even in the presence of black holes [328, 329, 397, 279, 202, 314, 224], the simplest and most transparent ones are probably those based on the use of 2-component spinors: If the dominant energy condition is satisfied on the $k$-asymptotically flat spacelike hypersurface $\Sigma$, where $k>\frac{1}{2}$, then the ADM energy-momentum is future pointing and non-spacelike (i.e. the Lorentzian length of the energy-momentum vector, the ADM mass, is non-negative), and it is null if and only if the domain of dependence $D(\Sigma)$ of $\Sigma$ is flat $[205,320,159,321,70]$. Its proof may be based on the Sparling equation $[345,130,313,266]: \nabla_{[a} u(\lambda, \bar{\mu})_{b c]}=-\frac{1}{2} \lambda_{E} \bar{\mu}_{E^{\prime}} G^{e f} \frac{1}{3 !} \varepsilon_{f a b c}+\Gamma(\lambda, \bar{\mu})_{a b c}$. The significance of this equation is that in the exterior derivative of the Nester-Witten 2-form the second derivatives of the metric appear only through the Einstein tensor, thus its structure is similar to that of the superpotential equations in the Lagrangian field theory, and $\Gamma(\lambda, \bar{\mu})_{a b c}$, known as the Sparling 3-form, is a quadratic expression of the first derivatives of the spinor fields. If the spinor fields $\lambda_{A}$ and $\mu_{A}$ solve the Witten equation on a spacelike hypersurface $\Sigma$, then the pull-back of $\Gamma(\lambda, \bar{\mu})_{a b c}$ to $\Sigma$ is positive definite. This theorem has been extended and refined in various ways, in particular by allowing inner boundaries of $\Sigma$ that represent future marginally trapped surfaces in black holes [159, 202, 314, 200].

The ADM energy-momentum can also be written as the 2-sphere integral of certain parts of the conformally rescaled spacetime curvature [15, 16, 28]. This expression is a special case of the more general 'Riemann tensor conserved quantities' (see [163]): If $\mathcal{S}$ is any closed spacelike 2-surface with area element $d \mathcal{S}$, then for any tensor fields $\omega_{a b}=\omega_{[a b]}$ and $\mu_{a b}=\mu_{[a b]}$ one can form the integral

$$
I_{\mathcal{S}}[\omega, \mu]:=\oint_{\mathcal{S}} \omega^{a b} R_{a b c d} \mu^{c d} d \mathcal{S} .
$$

Since the fall-off of the curvature tensor near spatial infinity is $r^{-k-2}$, the integral $I_{\mathcal{S}}[\omega, \mu]$ at spatial infinity can give finite value precisely when $\omega^{a b} \mu^{c d}$ blows up like $r^{k}$ as $r \rightarrow \infty$. In particular, for the $1 / r$ fall-off this condition can be satisfied by $\omega^{a b} \mu^{c d}=\sqrt{\operatorname{Area}(\mathcal{S})} \hat{\omega}^{a b} \hat{\mu}^{c d}$, where $\operatorname{Area}(\mathcal{S})$ is the area of $\mathcal{S}$ and the hatted tensor fields are $O(1)$.

If the spacetime is stationary, then the ADM energy can be recovered as the $r \rightarrow \infty$ limit of the 2-sphere integral of Komar's superpotential with the Killing vector $K^{a}$ of stationarity [163], too. On the other hand, if the spacetime is not stationary then, without additional restriction on the asymptotic time translation, the Komar expression does not reproduce the ADM energy. However, by Equations $(13,14)$ such an additional restriction might be that $K^{a}$ should be a constant combination of four future pointing null vector fields of the form $\alpha^{A} \bar{\alpha}^{A^{\prime}}$, where the spinor fields $\alpha^{A}$ are required to satisfy the Weyl neutrino equation $\nabla_{A^{\prime} A \alpha^{A}}=0$. This expression for the ADM energy-momentum was used to give an alternative, '4-dimensional' proof of the positivity of the ADM energy [205].

\subsubsection{Spatial infinity: Angular momentum}

The value of the Hamiltonian of Beig and Ó Murchadha [47] together with the appropriately defined asymptotic rotation-boost Killing vectors [364] define the spatial angular momentum and centre-of-mass, provided $k \geq 1$ and, in addition to the familiar fall-off conditions, certain global integral conditions are also satisfied. These integral conditions can be ensured by the explicit parity conditions of Regge and Teitelboim [319] on the leading nontrivial parts of the metric $h_{a b}$ and extrinsic curvature $\chi_{a b}$ : The components in the Cartesian coordinates $\left\{x^{\mathrm{i}}\right\}$ of the former must

Living Reviews in Relativity

http://www. livingreviews . org//rr-2004-4 
be even and the components of latter must be odd parity functions of $x^{\mathrm{i}} / r$ (see also [47]). Thus in what follows we assume that $k=1$. Then the value of the Beig-Ó Murchadha Hamiltonian parameterized by the asymptotic rotation Killing vectors is the spatial angular momentum of Regge and Teitelboim [319], while that parameterized by the asymptotic boost Killing vectors deviate from the centre-of-mass of Beig and Ó Murchadha [47] by a term which is the spatial momentum times the coordinate time. (As Beig and Ó Murchadha pointed out [47], the centre-ofmass of Regge and Teitelboim is not necessarily finite.) The spatial angular momentum and the new centre-of-mass form an anti-symmetric Lorentz 4-tensor, which transforms in the correct way under the 4-translation of the origin of the asymptotically Cartesian coordinate system, and it is conserved by the evolution equations [364].

The centre-of-mass of Beig and Ó Murchadha was reexpressed recently [42] as the $r \rightarrow \infty$ limit of 2-surface integrals of the curvature in the form (15) with $\omega^{a b} \mu^{c d}$ proportional to the lapse $N$ times $q^{a c} q^{b d}-q^{a d} q^{b c}$, where $q_{a b}$ is the induced 2-metric on $\mathcal{S}$ (see Section 4.1.1 below).

A geometric notion of centre-of-mass was introduced by Huisken and Yau [209]. They foliate the asymptotically flat hypersurface $\Sigma$ by certain spheres with constant mean curvature. By showing the global uniqueness of this foliation asymptotically, the origin of the leaves of this foliation in some flat ambient Euclidean space $\mathbb{R}^{3}$ defines the centre-of-mass (or rather 'centre-of-gravity') of Huisken and Yau. However, no statement on its properties is proven. In particular, it would be interesting to see whether or not this notion of centre-of-mass coincides, for example, with that of Beig and Ó Murchadha.

The Ashtekar-Hansen definition for the angular momentum is introduced in their specific conformal model of the spatial infinity as a certain 2-surface integral near infinity. However, their angular momentum expression is finite and unambiguously defined only if the magnetic part of the spacetime curvature tensor (with respect to the $\Omega=$ const. timelike level hypersurfaces of the conformal factor) falls off faster than would follow from the $1 / r$ fall-off of the metric (but they do not have to impose any global integral, e.g. a parity condition) [23, 15].

If the spacetime admits a Killing vector of axi-symmetry, then the usual interpretation of the corresponding Komar integral is the appropriate component of the angular momentum (see for example [387]). However, the value of the Komar integral is twice the expected angular momentum. In particular, if the Komar integral is normalized such that for the Killing field of stationarity in the Kerr solution the integral is $m / G$, for the Killing vector of axi-symmetry it is $2 m a / G$ instead of the expected $m a / G$ ('factor-of-two anomaly') [229]. We return to the discussion of the Komar integral in Section 12.1.

\subsubsection{Null infinity: Energy-momentum}

The study of the gravitational radiation of isolated sources led Bondi to the observation that the 2-sphere integral of a certain expansion coefficient $m(u, \theta, \phi)$ of the line element of a radiative spacetime in an asymptotically retarded spherical coordinate system $(u, r, \theta, \phi)$ behaves as the energy of the system at the retarded time $u$ : This notion of energy is not constant in time, but decreases with $u$, showing that gravitational radiation carries away positive energy ('Bondi's massloss') [71, 72]. The set of transformations leaving the asymptotic form of the metric invariant was identified as a group, nowadays known as the BMS group, having a structure very similar to that of the Poincaré group [325]. The only difference is that while the Poincaré group is a semidirect product of the Lorentz group and a 4-dimensional commutative group (of translations), the BMS group is the semidirect product of the Lorentz group and an infinite-dimensional commutative group, called the group of the supertranslations. A 4-parameter subgroup in the latter can be identified in a natural way as the group of the translations. Just at the same time the study of asymptotic solutions of the field equations led Newman and Unti to another concept of energy at null infinity [290]. However, this energy (nowadays known as the Newman-Unti energy) does not 
seem to have the same significance as the Bondi (or Bondi-Sachs [313] or Trautman-Bondi [115, 116, 114]) energy, because its monotonicity can be proven only between special, e.g. stationary, states. The Bondi energy, which is the time component of a Lorentz vector, the so-called BondiSachs energy-momentum, has a remarkable uniqueness property [115, 116].

Without additional conditions on $K^{a}$, Komar's expression does not reproduce the Bondi-Sachs energy-momentum in non-stationary spacetimes either [395, 163]: For the 'obvious' choice for $K^{a}$ Komar's expression yields the Newman-Unti energy. This anomalous behaviour in the radiative regime could be corrected in, at least, two ways. The first is by modifying the Komar integral according to

$$
L_{\mathcal{S}}[\mathbf{K}]:=\frac{1}{8 \pi G} \oint_{\mathcal{S}}\left(\nabla^{[c} K^{d]}+\alpha \nabla_{e} K^{e \perp} \varepsilon^{c d}\right) \frac{1}{2} \varepsilon_{c d a b}
$$

where ${ }^{\perp} \varepsilon_{c d}$ is the area 2-form on the Lorentzian 2-planes orthogonal to $\mathcal{S}$ (see Section 4.1.1) and $\alpha$ is some real constant. For $\alpha=1$ the integral $L_{\mathcal{S}}[\mathbf{K}]$, suggested by Winicour and Tamburino, is called the linkage [395]. In addition, to define physical quantities by linkages associated to a cut of the null infinity one should prescribe how the 2 -surface $\mathcal{S}$ tends to the cut and how the vector field $K^{a}$ should be propagated from the spacetime to null infinity into a BMS generator [395, 394]. The other way is considering the original Komar integral (i.e. $\alpha=0$ ) on the cut of infinity in the conformally rescaled spacetime and by requiring that $K^{a}$ be divergence-free [153]. For such asymptotic BMS translations both prescriptions give the correct expression for the Bondi-Sachs energy-momentum.

The Bondi-Sachs energy-momentum can also be expressed by the integral of the Nester-Witten 2-form [214, 255, 256, 205]. However, in non-stationary spacetimes the spinor fields that are asymptotically constant at null infinity are vanishing [83]. Thus the spinor fields in the NesterWitten 2-form must satisfy a weaker boundary condition at infinity such that the spinor fields themselves be the spinor constituents of the BMS translations. The first such condition, suggested by Bramson [83], was to require the spinor fields to be the solutions of the so-called asymptotic twistor equation (see Section 4.2.4). One can impose several such inequivalent conditions, and all these, based only on the linear first order differential operators coming from the two natural connections on the cuts (see Section 4.1.2), are determined in [363].

The Bondi-Sachs energy-momentum has a Hamiltonian interpretation as well. Although the fields on a spacelike hypersurface extending to null rather than spatial infinity do not form a closed system, a suitable generalization of the standard Hamiltonian analysis could be developed [114] and used to recover the Bondi-Sachs energy-momentum.

Similarly to the ADM case, the simplest proofs of the positivity of the Bondi energy [330] are probably those that are based on the Nester-Witten 2-form [214] and, in particular, the use of twocomponents spinors [255, 256, 205, 203, 321]: The Bondi-Sachs mass (i.e. the Lorentzian length of the Bondi-Sachs energy-momentum) of a cut of future null infinity is non-negative if there is a spacelike hypersurface $\Sigma$ intersecting null infinity in the given cut such that the dominant energy condition is satisfied on $\Sigma$, and the mass is zero iff the domain of dependence $D(\Sigma)$ of $\Sigma$ is flat.

\subsubsection{Null infinity: Angular momentum}

At null infinity there is no generally accepted definition for angular momentum, and there are various, mathematically inequivalent suggestions for it. Here we review only some of those total angular momentum definitions that can be considered as the null infinity limit of some quasi-local expression, and will be discussed in the main part of the review, namely in Section 9.

In their classic paper Bergmann and Thomson [60] raise the idea that while the gravitational energy-momentum is connected with the spacetime diffeomorphisms, the angular momentum should be connected with its intrinsic $O(1,3)$ symmetry. Thus, the angular momentum should be

Living Reviews in Relativity

http: //www. livingreviews.org/lrr-2004-4 
analogous with the spin. Based on the tetrad formalism of general relativity and following the prescription of constructing the Noether currents in Yang-Mills theories, Bramson suggested a superpotential for the six conserved currents corresponding to the internal Lorentz-symmetry $[84,85,86]$. (For another derivation of this superpotential from Møller's Lagrangian (9) see [363].) If $\left\{\lambda \frac{A}{A}\right\}$, $\underline{A}=0,1$, is a normalized spinor dyad corresponding to the orthonormal frame in Equation (9), then the integral of the spinor form of the anti-self-dual part of this superpotential on a closed orientable 2-surface $\mathcal{S}$ is

$$
J_{\mathcal{S}} \underline{A}:=\frac{1}{8 \pi G} \oint_{\mathcal{S}}-\mathrm{i} \lambda \frac{A}{(A} \lambda \frac{B}{B)} \varepsilon_{A^{\prime} B^{\prime}}
$$

where $\varepsilon_{A^{\prime} B^{\prime}}$ is the symplectic metric on the bundle of primed spinors. We will denote its integrand by $w\left(\lambda^{A}, \lambda \underline{B}\right)_{a b}$, and we call it the Bramson superpotential. To define angular momentum on a given cut of the null infinity by the formula (17) we should consider its limit when $\mathcal{S}$ tends to the cut in question and we should specify the spinor dyad, at least asymptotically. Bramson's suggestion for the spinor fields was to take the solutions of the asymptotic twistor equation [83]. He showed that this definition yields a well-defined expression, for stationary spacetimes this reduces to the generally accepted formula (34), and the corresponding Pauli-Lubanski spin, constructed from $\varepsilon^{\underline{A}^{\prime} \underline{B}^{\prime}} J \underline{A} \underline{B}+\varepsilon^{\underline{A}} \underline{B} \bar{J} \underline{A}^{\prime} \underline{B}^{\prime}$ and the Bondi-Sachs energy-momentum $P^{\underline{A}} \underline{A}^{\prime}$ (given for example in the Newman-Penrose formalism by Equation (33)), is invariant with respect to supertranslations of the cut ('active supertranslations'). Note that since Bramson's expression is based on the solutions of a system of partial differential equations on the cut in question, it is independent of the parameterization of the BMS vector fields. Hence, in particular, it is invariant with respect to the supertranslations of the origin cut ('passive supertranslations'). Therefore, Bramson's global angular momentum behaves like the spin part of the total angular momentum.

The construction based on the Winicour-Tamburino linkage (16) can be associated with any BMS vector field $[395,252,30]$. In the special case of translations it reproduces the Bondi-Sachs energy-momentum. The quantities that it defines for the proper supertranslations are called the super-momenta. For the boost-rotation vector fields they can be interpreted as angular momentum. However, in addition to the factor-of-two anomaly, this notion of angular momentum contains a huge ambiguity ('supertranslation ambiguity'): The actual form of both the boost-rotation Killing vector fields of Minkowski spacetime and the boost-rotation BMS vector fields at future null infinity depend on the choice of origin, a point in Minkowski spacetime and a cut of null infinity, respectively. However, while the set of the origins of Minkowski spacetime is parameterized by four numbers, the set of the origins at null infinity requires a smooth function of the form $u: S^{2} \rightarrow \mathbb{R}$. Consequently, while the corresponding angular momentum in the Minkowski spacetime has the familiar origin-dependence (containing four parameters), the analogous transformation of the angular momentum defined by using the boost-rotation BMS vector fields depends on an arbitrary smooth real valued function on the 2-sphere. This makes the angular momentum defined at null infinity by the boost-rotation BMS vector fields ambiguous unless a natural selection rule for the origins, making them form a four parameter family of cuts, is found. Such a selection rule could be the suggestion by Dain and Moreschi [125] in the charge integral approach to angular momentum of Moreschi [272, 273].

Another promising approach might be that of Chruściel, Jezierski, and Kijowski [114], which is based on a Hamiltonian analysis of general relativity on asymptotically hyperbolic spacelike hypersurfaces. They chose the six BMS vector fields tangent to the intersection of the spacelike hypersurface and null infinity as the generators of their angular momentum. Since the motions that their angular momentum generators define leave the domain of integration fixed, and apparently there is no Lorentzian 4-space of origins, they appear to be the generators with respect to some fixed 'centre-of-the-cut', and the corresponding angular momentum appears to be the intrinsic angular momentum. 


\subsection{The necessity of quasi-locality for the observables in general rela- tivity}

\subsubsection{Non-locality of the gravitational energy-momentum and angular momentum}

One reaction to the non-tensorial nature of the gravitational energy-momentum density expressions was to consider the whole problem ill-defined and the gravitational energy-momentum meaningless. However, the successes discussed in the previous Section 3.2.4 show that the global gravitational energy-momenta and angular momenta are useful notions, and hence it could also be useful to introduce them even if the spacetime is not asymptotically flat. Furthermore, the non-tensorial nature of an object does not imply that it is meaningless. For example, the Christoffel symbols are not tensorial, but they do have geometric, and hence physical content, namely the linear connection. Indeed, the connection is a non-local geometric object, connecting the fibres of the vector bundle over different points of the base manifold. Hence any expression of the connection coefficients, in particular the gravitational energy-momentum or angular momentum, must also be non-local. In fact, although the connection coefficients at a given point can be taken zero by an appropriate coordinate/gauge transformation, they cannot be transformed to zero on an open domain unless the connection is flat.

Furthermore, the superpotential of many of the classical pseudotensors (e.g. of the Einstein, Bergmann, Møller's tetrad, Landau-Lifshitz pseudotensors), being linear in the connection coefficients, can be recovered as the pull-back to the spacetime manifold of various forms of a single geometric object on the linear frame bundle, namely of the Nester-Witten 2-form, along various local Sections [142, 266, 352, 353], and the expression of the pseudotensors by their superpotentials are the pull-backs of the Sparling equation [345, 130, 266]. In addition, Chang, Nester, and Chen [104] found a natural quasi-local Hamiltonian interpretation of each of the pseudotensorial expressions in the metric formulation of the theory (see Section 11.3.4). Therefore, the pseudotensors appear to have been 'rehabilitated', and the gravitational energy-momentum and angular momentum are necessarily associated with extended subsets of the spacetime.

This fact is a particular consequence of a more general phenomenon [324, 213]: Since the physical spacetime is the isomorphism class of the pairs $\left(M, g_{a b}\right)$ instead of a single such pair, it is meaningless to speak about the 'value of a scalar or vector field at a point $p \in M$ '. What could have meaning are the quantities associated with curves (the length of a curve, or the holonomy along a closed curve), 2-surfaces (e.g. the area of a closed 2-surface) etc. determined by some body or physical fields. Thus, if we want to associate energy-momentum and angular momentum not only to the whole (necessarily asymptotically flat) spacetime, then these quantities must be associated with extended but finite subsets of the spacetime, i.e. must be quasi-local.

\subsubsection{Domains for quasi-local quantities}

The quasi-local quantities (usually the integral of some local expression of the field variables) are associated with a certain type of subset of spacetime. In four dimensions there are three natural candidates:

1. the globally hyperbolic domains $D \subset M$ with compact closure,

2. the compact spacelike hypersurfaces $\Sigma$ with boundary (interpreted as Cauchy surfaces for globally hyperbolic domains $D$ ), and

3. the closed, orientable spacelike 2 -surfaces $\mathcal{S}$ (interpreted as the boundary $\partial \Sigma$ of Cauchy surfaces for globally hyperbolic domains).

A typical example fo Type 3 is any charge integral expression: The quasi-local quantity is the integral of some superpotential 2-form built from the data given on the 2-surface, as in Equation (12),

Living Reviews in Relativity

http: //www . livingreviews . org/lrr-2004-4 
or the expression $Q_{\mathcal{S}}[\mathbf{K}]$ for the matter fields given by Equation (5). An example for Type 2 might be the integral of the Bel-Robinson 'momentum' on the hypersurface $\Sigma$ :

$$
E_{\Sigma}\left[\xi^{a}\right]:=\int_{\Sigma} \xi^{d} T_{d e f g} t^{e} t^{f} \frac{1}{3 !} \varepsilon^{g}{ }_{a b c} .
$$

This quantity is analogous to the integral $E_{\Sigma}\left[\xi^{a}\right]$ for the matter fields given by Equation (6) (though, by the remarks on the Bel-Robinson 'energy' in Section 3.1.1, its physical dimension cannot be of energy). If $\xi^{a}$ is a future pointing nonspacelike vector then $E_{\Sigma}\left[\xi^{a}\right] \geq 0$. Obviously, if such a quantity were independent of the actual hypersurface $\Sigma$, then it could also be rewritten as a charge integral on the boundary $\partial \Sigma$. The gravitational Hamiltonian provides an interesting example for the mixture of Type 2 and 3 expressions, because the form of the Hamiltonian is the 3 -surface integral of the constraints on $\Sigma$ and a charge integral on its boundary $\partial \Sigma$, thus if the constraints are satisfied then the Hamiltonian reduces to a charge integral. Finally, an example for Type 1 might be

$$
E_{D}:=\inf \left\{E_{\Sigma}[\mathbf{t}] \mid \Sigma \text { is a Cauchy surface for } D\right\},
$$

the infimum of the 'quasi-local Bel-Robinson energies', where the infimum is taken on the set of all the Cauchy surfaces $\Sigma$ for $D$ with given boundary $\partial \Sigma$. (The infimum always exists because the Bel-Robinson 'energy density' $T_{a b c d} t^{a} t^{b} t^{c} t^{d}$ is non-negative.) Quasi-locality in any of these three senses agrees with the quasi-locality of Haag and Kastler [168, 169]. The specific quasi-local energymomentum constructions provide further examples both for charge-integral-type expressions and those based on spacelike hypersurfaces.

\subsubsection{Strategies to construct quasi-local quantities}

There are two natural ways of finding the quasi-local energy-momentum and angular momentum. The first is to follow some systematic procedure, while the second is the 'quasi-localization' of the global energy-momentum and angular momentum expressions. One of the two systematic procedures could be called the Lagrangian approach: The quasi-local quantities are integrals of some superpotential derived from the Lagrangian via a Noether-type analysis. The advantage of this approach could be its manifest Lorentz-covariance. On the other hand, since the Noether current is determined only through the Noether identity, which contains only the divergence of the current itself, the Noether current and its superpotential is not uniquely determined. In addition (as in any approach), a gauge reduction (for example in the form of a background metric or reference configuration) and a choice for the 'translations' and 'boost-rotations' should be made.

The other systematic procedure might be called the Hamiltonian approach: At the end of a fully quasi-local (covariant or not) Hamiltonian analysis we would have a Hamiltonian, and its value on the constraint surface in the phase space yields the expected quantities. Here the main idea is that of Regge and Teitelboim [319] that the Hamiltonian must reproduce the correct field equations as the flows of the Hamiltonian vector fields, and hence, in particular, the correct Hamiltonian must be functionally differentiable with respect to the canonical variables. This differentiability may restrict the possible 'translations' and 'boost-rotations' too. However, if we are not interested in the structure of the quasi-local phase space, then, as a short-cut, we can use the Hamilton-Jacobi method to define the quasi-local quantities. The resulting expression is a 2-surface integral. Nevertheless, just as in the Lagrangian approach, this general expression is not uniquely determined, because the action can be modified by adding an (almost freely chosen) boundary term to it. Furthermore, the 'translations' and 'boost-rotations' are still to be specified.

On the other hand, at least from a pragmatic point of view, the most natural strategy to introduce the quasi-local quantities would be some 'quasi-localization' of those expressions that gave the global energy-momentum and angular momentum of asymptotically flat spacetimes. Therefore, 
respecting both strategies, it is also legitimate to consider the Winicour-Tamburino-type (linkage) integrals and the charge integrals of the curvature.

Since the global energy-momentum and angular momentum of asymptotically flat spacetimes can be written as 2-surface integrals at infinity (and, as we will see in Section 7.1.1, both the energymomentum and angular momentum of the source in the linear approximation and the gravitational mass in the Newtonian theory of gravity can also be written as 2-surface integrals), the 2-surface observables can be expected to have special significance. Thus, to summarize, if we want to define reasonable quasi-local energy-momentum and angular momentum as 2 -surface observables, then three things must be specified:

1. an appropriate general 2-surface integral (e.g. the integral of a superpotential 2-form in the Lagrangian approaches or a boundary term in the Hamiltonian approaches),

2. a gauge choice (in the form of a distinguished coordinate system in the pseudotensorial approaches, or a background metric/connection in the background field approaches or a distinguished tetrad field in the tetrad approach), and

3. a definition for the 'quasi-symmetries' of the 2-surface (i.e. the 'generator vector fields' of the quasi-local quantities in the Lagrangian, and the lapse and the shift in the Hamiltonian approaches, respectively, which, in the case of timelike 'generator vector fields', can also be interpreted as a fleet of observers on the 2-surface).

In certain approaches the definition of the 'quasi-symmetries' is linked to the gauge choice, for example by using the Killing symmetries of the flat background metric.

Living Reviews in Relativity

http: //www . livingreviews . org/lrr-2004-4 


\section{Tools to Construct and Analyze the Quasi-Local Quanti- ties}

Having accepted that the gravitational energy-momentum and angular momentum should be introduced at the quasi-local level, we next need to discuss the special tools and concepts that are needed in practice to construct (or even to understand the various special) quasi-local expressions. Thus, first, in Section 4.1 we review the geometry of closed spacelike 2-surfaces, with special emphasis on the so-called 2-surface data. Then, in the remaining two Sections 4.2 and 4.3, we discuss the special situations where there is a more or less generally accepted 'standard' definition for the energy-momentum (or at least for the mass) and angular momentum. In these situations any reasonable quasi-local quantity should reduce to them.

\subsection{The geometry of spacelike 2-surfaces}

The first systematic study of the geometry of spacelike 2-surfaces from the point of view of quasilocal quantities is probably due to Tod [375, 380]. Essentially, his approach is based on the GHP (Geroch-Held-Penrose) formalism [152]. Although this is a very effective and flexible formalism [152, 312, 313, 206, 347], its form is not spacetime covariant. Since in many cases the covariance of a formalism itself already gives some hint how to treat and solve the problem at hand, here we concentrate mainly on a spacetime-covariant description of the geometry of the spacelike 2-surfaces, developed gradually in [355, 357, 358, 359, 147]. The emphasis will be on the geometric structures rather than the technicalities. In the last paragraph, we comment on certain objects appearing in connection with families of spacelike 2-surfaces.

\subsubsection{The Lorentzian vector bundle}

The restriction $\mathbf{V}^{a}(\mathcal{S})$ to the closed, orientable spacelike 2-surface $\mathcal{S}$ of the tangent bundle $T M$ of the spacetime has a unique decomposition to the $g_{a b}$-orthogonal sum of the tangent bundle $T \mathcal{S}$ of $\mathcal{S}$ and the bundle of the normals, denoted by $N \mathcal{S}$. Then all the geometric structures of the spacetime (metric, connection, curvature) can be decomposed in this way. If $t^{a}$ and $v^{a}$ are timelike and spacelike unit normals, respectively, being orthogonal to each other, then the projection to $T \mathcal{S}$ and $N \mathcal{S}$ is $\Pi_{b}^{a}:=\delta_{b}^{a}-t^{a} t_{b}+v^{a} v_{b}$ and $O_{b}^{a}:=\delta_{b}^{a}-\Pi_{b}^{a}$, respectively. The induced 2-metric and the corresponding area 2 -form on $\mathcal{S}$ will be denoted by $q_{a b}=g_{a b}-t_{a} t_{b}+v_{a} v_{b}$ and $\varepsilon_{a b}=t^{c} v^{d} \varepsilon_{c d a b}$, respectively, while the area 2 -form on the normal bundle will be ${ }^{\perp} \varepsilon_{a b}=t_{a} v_{b}-t_{b} v_{a}$. The bundle $\mathbf{V}^{a}(\mathcal{S})$ together with the fibre metric $g_{a b}$ and the projection $\Pi_{b}^{a}$ will be called the Lorentzian vector bundle over $\mathcal{S}$. For the discussion of the global topological properties of the closed orientable 2manifolds, see for example [5].

\subsubsection{Connections}

The spacetime covariant derivative operator $\nabla_{e}$ defines two covariant derivatives on $\mathbf{V}^{a}(\mathcal{S})$. The first, denoted by $\delta_{e}$, is analogous to the induced (intrinsic) covariant derivative on (one-codimensional) hypersurfaces: $\delta_{e} X^{a}:=\Pi_{b}^{a} \Pi_{e}^{f} \nabla_{f}\left(\Pi_{c}^{b} X^{c}\right)+O_{b}^{a} \Pi_{e}^{f} \nabla_{f}\left(O_{c}^{b} X^{c}\right)$ for any section $X^{a}$ of $\mathbf{V}^{a}(\mathcal{S})$. Obviously, $\delta_{e}$ annihilates both the fibre metric $g_{a b}$ and the projection $\Pi_{b}^{a}$. However, since for 2-surfaces in four dimensions the normal is not uniquely determined, we have the 'boost gauge freedom' $t^{a} \mapsto t^{a} \cosh u+v^{a} \sinh u, v^{a} \mapsto t^{a} \sinh u+v^{a} \cosh u$. The induced connection will have a nontrivial part on the normal bundle, too. The corresponding (normal part of the) connection 1-form on $\mathcal{S}$ can be characterized, for example, by $A_{e}:=\Pi_{e}^{f}\left(\nabla_{f} t_{a}\right) v^{a}$. Therefore, the connection $\delta_{e}$ can be considered as a connection on $\mathbf{V}^{a}(\mathcal{S})$ coming from a connection on the $O(2) \otimes O(1,1)$-principal bundle of the $g_{a b}$-orthonormal frames adapted to $\mathcal{S}$. 
The other connection, $\Delta_{e}$, is analogous to the Sen connection [331], and is defined simply by $\Delta_{e} X^{a}:=\Pi_{e}^{f} \nabla_{f} X^{a}$. This annihilates only the fibre metric, but not the projection. The difference of the connections $\Delta_{e}$ and $\delta_{e}$ turns out to be just the extrinsic curvature tensor: $\Delta_{e} X^{a}=$ $\delta_{e} X^{a}+Q^{a}{ }_{e b} X^{b}-X^{b} Q_{b e}{ }^{a}$. Here $Q^{a}{ }_{e b}:=-\Pi_{c}^{a} \Delta_{e} \Pi_{b}^{c}=\tau^{a}{ }_{e} t_{b}-\nu^{a}{ }_{e} v_{b}$, and $\tau_{a b}:=\Pi_{a}^{c} \Pi_{b}^{d} \nabla_{c} t_{d}$ and $\nu_{a b}:=\Pi_{a}^{c} \Pi_{b}^{d} \nabla_{c} v_{d}$ are the standard (symmetric) extrinsic curvatures corresponding to the individual normals $t_{a}$ and $v_{a}$, respectively. The familiar expansion tensors of the future pointing outgoing and ingoing null normals, $l^{a}:=t^{a}+v^{a}$ and $n^{a}:=\frac{1}{2}\left(t^{a}-v^{a}\right)$, respectively, are $\theta_{a b}=Q_{a b c} l^{c}$ and $\theta_{a b}^{\prime}=Q_{a b c} n^{c}$, and the corresponding shear tensors $\sigma_{a b}$ and $\sigma_{a b}^{\prime}$ are defined by their trace-free part. Obviously, $\tau_{a b}$ and $\nu_{a b}$ (and hence the expansion and shear tensors $\theta_{a b}, \theta_{a b}^{\prime}, \sigma_{a b}$, and $\sigma_{a b}^{\prime}$ ) are boost-gauge dependent quantities (and it is straightforward to derive their transformation from the definitions), but their combination $Q^{a}{ }_{e b}$ is boost-gauge invariant. In particular, it defines a natural normal vector field to $\mathcal{S}$ by $Q_{b}:=Q^{a}{ }_{a b}=\tau t_{b}-\nu v_{b}=\theta^{\prime} l_{b}+\theta n_{b}$, where $\tau, \nu, \theta$ and $\theta^{\prime}$ are the relevant traces. $Q_{a}$ is called the main extrinsic curvature vector of $\mathcal{S}$. If $\tilde{Q}_{b}:=\nu t_{b}-\tau v_{b}=-\theta^{\prime} l_{a}+\theta n_{a}$, then the norm of $Q_{a}$ and $\tilde{Q}_{a}$ is $Q_{a} Q_{b} g^{a b}=-\tilde{Q}_{a} \tilde{Q}_{b} g^{a b}=\tau^{2}-\nu^{2}=2 \theta \theta^{\prime}$, and they are orthogonal to each other: $Q_{a} \tilde{Q}_{b} g^{a b}=0$. It is easy to show that $\Delta_{a} \tilde{Q}^{a}=0$, i.e. $\tilde{Q}^{a}$ is the uniquely pointwise determined direction orthogonal to the 2-surface in which the expansion of the surface is vanishing. If $Q_{a}$ is not null, then $\left\{Q_{a}, \tilde{Q}_{a}\right\}$ defines an orthonormal frame in the normal bundle (see for example [8]). If $Q_{a}$ is non-zero but (e.g. future pointing) null, then there is a uniquely determined null normal $S_{a}$ to $\mathcal{S}$ such that $Q_{a} S^{a}=1$, and hence $\left\{Q_{a}, S_{a}\right\}$ is a uniquely determined null frame. Therefore, the 2 -surface admits a natural gauge choice in the normal bundle unless $Q_{a}$ is vanishing. Geometrically, $\Delta_{e}$ is a connection coming from a connection on the $O(1,3)$-principal fibre bundle of the $g_{a b}$-orthonormal frames. The curvature of the connections $\delta_{e}$ and $\Delta_{e}$, respectively, are

$$
\begin{aligned}
f^{a}{ }_{b c d}= & -{ }^{\perp} \varepsilon^{a}{ }_{b}\left(\delta_{c} A_{d}-\delta_{d} A_{c}\right)+{ }^{\frac{1}{2}}{ }^{\mathcal{S}} R\left(\Pi_{c}^{a} q_{b d}-\Pi_{d}^{a} q_{b c}\right), \\
F^{a}{ }_{b c d}= & f^{a}{ }_{b c d}-\delta_{c}\left(Q^{a}{ }_{d b}-Q_{b d}{ }^{a}\right)+\delta_{d}\left(Q^{a}{ }_{c b}-Q_{b c}{ }^{a}\right)+ \\
& +Q^{a}{ }_{c e} Q_{b d}{ }^{e}+Q_{e c}{ }^{a} Q^{e}{ }_{d b}-Q^{a}{ }_{d e} Q_{b c}{ }^{e}-Q_{e d}{ }^{a} Q^{e}{ }_{c b},
\end{aligned}
$$

where ${ }^{\mathcal{S}} R$ is the curvature scalar of the familiar intrinsic Levi-Civita connection of $\left(\mathcal{S}, q_{a b}\right)$. The curvature of $\Delta_{e}$ is just the pull-back to $\mathcal{S}$ of the spacetime curvature 2 -form: $F^{a}{ }_{b c d}=R^{a}{ }_{b e f} \Pi_{c}^{e} \Pi_{d}^{f}$. Therefore, the well known Gauss, Codazzi-Mainardi, and Ricci equations for the embedding of $\mathcal{S}$ in $M$ are just the various projections of Equation (21).

\subsubsection{Convexity conditions}

To prove certain statements on quasi-local quantities various forms of the convexity of $\mathcal{S}$ must be assumed. The convexity of $\mathcal{S}$ in a 3 -geometry is defined by the positive definiteness of its extrinsic curvature tensor. If the embedding space is flat, then by the Gauss equation this is equivalent to the positivity of the scalar curvature of the intrinsic metric of $\mathcal{S}$. If $\mathcal{S}$ is in a Lorentzian spacetime then the weakest convexity conditions are conditions only on the mean null curvatures: $\mathcal{S}$ will be called weakly future convex if the outgoing null normals $l^{a}$ are expanding on $\mathcal{S}$, i.e. $\theta:=q^{a b} \theta_{a b}>0$, and weakly past convex if $\theta^{\prime}:=q^{a b} \theta_{a b}^{\prime}<0$ [380]. $\mathcal{S}$ is called mean convex [182] if $\theta \theta^{\prime}<0$ on $\mathcal{S}$, or, equivalently, if $\tilde{Q}_{a}$ is timelike. To formulate stronger convexity conditions we must consider the determinant of the null expansions $D:=\operatorname{det}\left\|\theta_{b}^{a}\right\|=\frac{1}{2}\left(\theta_{a b} \theta_{c d}-\theta_{a c} \theta_{b d}\right) q^{a b} q^{c d}$ and $D^{\prime}:=\operatorname{det}\left\|\theta^{\prime a}{ }_{b}\right\|=\frac{1}{2}\left(\theta_{a b}^{\prime} \theta_{c d}^{\prime}-\theta_{a c}^{\prime} \theta_{b d}^{\prime}\right) q^{a b} q^{c d}$. Note that although the expansion tensors, and in particular the functions $\theta, \theta^{\prime}, D$, and $D^{\prime}$ are gauge dependent, their sign is gauge invariant. Then $\mathcal{S}$ will be called future convex if $\theta>0$ and $D>0$, and past convex if $\theta^{\prime}<0$ and $D^{\prime}>0$ [380,358]. These are equivalent to the requirement that the two eigenvalues of $\theta^{a}{ }_{b}$ be positive and those of $\theta^{\prime a}{ }_{b}$ be negative everywhere on $\mathcal{S}$, respectively. A different kind of convexity condition, based on global concepts, will be used in Section 6.1.3.

Living Reviews in Relativity

http: //www. livingreviews . org//rr-2004-4 


\subsubsection{The spinor bundle}

The connections $\delta_{e}$ and $\Delta_{e}$ determine connections on the pull-back $\mathbf{S}^{A}(\mathcal{S})$ to $\mathcal{S}$ of the bundle of unprimed spinors. The natural decomposition $\mathbf{V}^{a}(\mathcal{S})=T \mathcal{S} \oplus N \mathcal{S}$ defines a chirality on the spinor bundle $\mathbf{S}^{A}(\mathcal{S})$ in the form of the spinor $\gamma^{A}{ }_{B}:=2 t^{A A^{\prime}} v_{B A^{\prime}}$, which is analogous to the $\gamma_{5}$ matrix in the theory of Dirac spinors. Then the extrinsic curvature tensor above is a simple expression of $Q^{A}{ }_{e B}:=\frac{1}{2}\left(\Delta_{e} \gamma^{A}{ }_{C}\right) \gamma^{C}{ }_{B}$ and $\gamma^{A}{ }_{B}$ (and their complex conjugate), and the two covariant derivatives on $\mathbf{S}^{A}(\mathcal{S})$ are related to each other by $\Delta_{e} \lambda^{A}=\delta_{e} \lambda^{A}+Q^{A}{ }_{e B} \lambda^{B}$. The curvature $F^{A}{ }_{B c d}$ of $\Delta_{e}$ can be expressed by the curvature $f_{B c d}^{A}$ of $\delta_{e}$, the spinor $Q^{A}{ }_{e B}$, and its $\delta_{e}$-derivative. We can form the scalar invariants of the curvatures according to

$$
\begin{aligned}
& f:=f_{a b c d} \frac{1}{2}\left(\varepsilon^{a b}-\mathrm{i}^{\perp} \varepsilon^{a b}\right) \varepsilon^{c d}=\mathrm{i} \gamma_{B}^{A} f_{A c d}^{B} \varepsilon^{c d}={ }^{\mathcal{S}} R-2 \mathrm{i} \delta_{c}\left(\varepsilon^{c d} A_{d}\right), \\
& F:=F_{a b c d} \frac{1}{2}\left(\varepsilon^{a b}-\mathrm{i}^{\perp} \varepsilon^{a b}\right) \varepsilon^{c d}=\mathrm{i} \gamma_{B}^{A} F^{B}{ }_{A c d} \varepsilon^{c d}=f+\theta \theta^{\prime}-2 \sigma_{e a}^{\prime} \sigma_{b}^{e}\left(q^{a b}+\mathrm{i} \varepsilon^{a b}\right) .
\end{aligned}
$$

$f$ is four times the so-called complex Gauss curvature [312] of $\mathcal{S}$, by means of which the whole curvature $f_{B c d}^{A}$ can be characterized: $f^{A}{ }_{B c d}=-\frac{\mathrm{i}}{4} f \gamma^{A}{ }_{B} \varepsilon_{c d}$. If the spacetime is space and time orientable, at least on an open neighbourhood of $\mathcal{S}$, then the normals $t_{a}$ and $v_{a}$ can be chosen to be globally well-defined, and hence $N \mathcal{S}$ is globally trivializable and the imaginary part of $f$ is a total divergence of a globally well-defined vector field.

An interesting decomposition of the $S O(1,1)$ connection 1-form $A_{e}$, i.e. the vertical part of the connection $\delta_{e}$, was given by Liu and Yau [253]: There are real functions $\alpha$ and $\gamma$, unique up to additive constants, such that $A_{e}=\varepsilon_{e}{ }^{f} \delta_{f} \alpha+\delta_{e} \gamma . \alpha$ is globally defined on $\mathcal{S}$, but in general $\gamma$ is defined only on the local trivialization domains of $N \mathcal{S}$ that are homeomorphic to $\mathbb{R}^{2}$. It is globally defined if $H^{1}(\mathcal{S})=0$. In this decomposition $\alpha$ is the boost-gauge invariant part of $A_{e}$, while $\gamma$ represents its gauge content. Since $\delta_{e} A^{e}=\delta_{e} \delta^{e} \gamma$, the 'Coulomb-gauge condition' $\delta_{e} A^{e}=0$ uniquely fixes $A_{e}$ (see also Section 10.4.1).

By the Gauss-Bonnet theorem $\oint_{\mathcal{S}} f d \mathcal{S}=\oint_{\mathcal{S}} \mathcal{S}_{R} d \mathcal{S}=8 \pi(1-g)$, where $g$ is the genus of $\mathcal{S}$. Thus geometrically the connection $\delta_{e}$ is rather poor, and can be considered as a part of the "universal structure of $\mathcal{S}$ '. On the other hand, the connection $\Delta_{e}$ is much richer, and, in particular, the invariant $F$ carries information on the mass aspect of the gravitational 'field'. The 2-surface data for charge-type quasi-local quantities (i.e. for 2-surface observables) are the universal structure (i.e. the intrinsic metric $q_{a b}$, the projection $\Pi_{b}^{a}$ and the connection $\delta_{e}$ ) and the extrinsic curvature tensor $Q^{a}{ }_{e b}$.

\subsubsection{Curvature identities}

The complete decomposition of $\Delta_{A A^{\prime}} \lambda_{B}$ into its irreducible parts gives $\Delta_{A^{\prime} A^{A}} \lambda^{A}$, the Dirac-Witten operator, and $\mathcal{T}_{E^{\prime} E A}{ }^{B} \lambda_{B}:=\Delta_{E^{\prime}(E} \lambda_{A)}+\frac{1}{2} \gamma_{E A} \gamma^{C D} \Delta_{E^{\prime} C} \lambda_{D}$, the 2-surface twistor operator. A Sen-Witten-type identity for these irreducible parts can be derived. Taking its integral one has

$$
\oint_{\mathcal{S}} \bar{\gamma}^{A^{\prime} B^{\prime}}\left[\left(\Delta_{A^{\prime} A} \lambda^{A}\right)\left(\Delta_{B^{\prime} B} \mu^{B}\right)+\left(\mathcal{T}_{A^{\prime} C D}{ }^{E} \lambda_{E}\right)\left(\mathcal{T}_{B^{\prime}}{ }^{C D F} \mu_{F}\right)\right] d \mathcal{S}=-\frac{\mathrm{i}}{2} \oint_{\mathcal{S}} \lambda^{A} \mu^{B} F_{A B c d}
$$

where $\lambda_{A}$ and $\mu_{A}$ are two arbitrary spinor fields on $\mathcal{S}$, and the right hand side is just the charge integral of the curvature $F^{A}{ }_{B c d}$ on $\mathcal{S}$.

\subsubsection{The GHP formalism}

A GHP spin frame on the 2-surface $\mathcal{S}$ is a normalized spinor basis $\varepsilon_{\mathbf{A}}^{A}:=\left\{o^{A}, \iota^{A}\right\}, \mathbf{A}=0,1$, such that the complex null vectors $m^{a}:=o^{A} \bar{\iota}^{A^{\prime}}$ and $\bar{m}^{a}:=\iota^{A} \bar{o}^{A^{\prime}}$ are tangent to $\mathcal{S}$ (or, equivalently, the future pointing null vectors $l^{a}:=o^{A} \bar{o}^{A^{\prime}}$ and $n^{a}:=\iota^{A} \bar{\iota}^{A^{\prime}}$ are orthogonal to $\mathcal{S}$ ). Note, however, that 
László B. Szabados

in general a GHP spin frame can be specified only locally, but not globally on the whole $\mathcal{S}$. This fact is connected with the non-triviality of the tangent bundle $T \mathcal{S}$ of the 2 -surface. For example, on the 2-sphere every continuous tangent vector field must have a zero, and hence, in particular, the vectors $m^{a}$ and $\bar{m}^{a}$ cannot form a globally defined basis on $\mathcal{S}$. Consequently, the GHP spin frame cannot be globally defined either. The only closed orientable 2 -surface with globally trivial tangent bundle is the torus.

Fixing a GHP spin frame $\left\{\varepsilon_{\mathbf{A}}^{A}\right\}$ on some open $U \subset \mathcal{S}$, the components of the spinor and tensor fields on $U$ will be local representatives of cross sections of appropriate complex line bundles $E(p, q)$ of scalars of type $(p, q)[152,312]$ : A scalar $\phi$ is said to be of type $(p, q)$ if under the rescaling $o^{A} \mapsto$ $\lambda o^{A}, \iota^{A} \mapsto \lambda^{-1} \iota^{A}$ of the GHP spin frame with some nowhere vanishing complex function $\lambda: U \rightarrow \mathbb{C}$ the scalar transforms as $\phi \mapsto \lambda^{p} \bar{\lambda}^{q} \phi$. For example $\rho:=\theta_{a b} m^{a} \bar{m}^{b}=-\frac{1}{2} \theta, \rho^{\prime}:=\theta_{a b}^{\prime} m^{a} \bar{m}^{b}=-\frac{1}{2} \theta^{\prime}$, $\sigma:=\theta_{a b} m^{a} m^{b}=\sigma_{a b} m^{a} m^{b}$, and $\sigma^{\prime}:=\theta_{a b}^{\prime} \bar{m}^{a} \bar{m}^{b}=\sigma_{a b}^{\prime} \bar{m}^{a} \bar{m}^{b}$ are of type $(1,1),(-1,-1),(3,-1)$, and $(-3,1)$, respectively. The components of the Weyl and Ricci spinors, $\psi_{0}:=\psi_{A B C D} O^{A} O^{B} O^{C} O^{D}$, $\psi_{1}:=\psi_{A B C D} O^{A} O^{B} O^{C} \iota^{D}, \psi_{2}:=\psi_{A B C D} O^{A} O^{B} \iota^{C} \iota^{D}, \ldots, \phi_{00}:=\phi_{A B^{\prime}} O^{A} \bar{o}^{B^{\prime}}, \phi_{01}:=\phi_{A B^{\prime}} O^{A} \bar{\iota}^{B^{\prime}}, \ldots$, etc., also have definite $(p, q)$-type. In particular, $\Lambda:=R / 24$ has type $(0,0)$. A global section of $E(p, q)$ is a collection of local cross sections $\left\{(U, \phi),\left(U^{\prime}, \phi^{\prime}\right), \ldots\right\}$ such that $\left\{U, U^{\prime}, \ldots\right\}$ forms a covering of $\mathcal{S}$ and on the non-empty overlappings, e.g. on $U \cap U^{\prime}$ the local sections are related to each other by $\phi=\psi^{p} \bar{\psi}^{q} \phi^{\prime}$, where $\psi: U \cap U^{\prime} \rightarrow \mathbb{C}$ is the transition function between the GHP spin frames: $o^{A}=\psi o^{\prime A}$ and $\iota^{A}=\psi^{-1} \iota^{\prime A}$.

The connection $\delta_{e}$ defines a connection $\partial_{e}$ on the line bundles $E(p, q)[152,312]$. The usual edth operators, $\varnothing$ and $\chi^{\prime}$, are just the directional derivatives $\delta:=m^{a} \partial_{a}$ and $\chi^{\prime}:=\bar{m}^{a} \gamma_{a}$ on the domain $U \subset \mathcal{S}$ of the GHP spin frame $\left\{\varepsilon_{\mathbf{A}}^{A}\right\}$. These locally defined operators yield globally defined differential operators, denoted also by $\chi$ and ${ }^{\prime}$, on the global sections of $E(p, q)$. It might be worth emphasizing that the GHP spin coefficients $\beta$ and $\beta^{\prime}$, which do not have definite $(p, q)$-type, play the role of the two components of the connection 1-form, and they are built both from the connection 1-form for the intrinsic Riemannian geometry of $\left(\mathcal{S}, q_{a b}\right)$ and the connection 1-form $A_{e}$ in the normal bundle. $\delta$ and $\partial^{\prime}$ are elliptic differential operators, thus their global properties, e.g. the dimension of their kernel, are connected with the global topology of the line bundle they act on, and, in particular, with the global topology of $\mathcal{S}$. These properties are discussed in [147] for general, and in $[132,43,356]$ for spherical topology.

\subsubsection{Irreducible parts of the derivative operators}

Using the projection operators $\pi^{ \pm A}{ }_{B}:=\frac{1}{2}\left(\delta_{B}^{A} \pm \gamma^{A}{ }_{B}\right)$, the irreducible parts $\Delta_{A^{\prime} A} \lambda^{A}$ and $\mathcal{T}_{E^{\prime} E A}{ }^{B} \lambda_{B}$ can be decomposed further into their right handed and left handed parts. In the GHP formalism these chiral irreducible parts are

$$
\begin{aligned}
-\Delta^{-} \lambda & :=ð \lambda_{1}+\rho^{\prime} \lambda_{0}, & \Delta^{+} \lambda & :=\chi^{\prime} \lambda_{0}+\rho \lambda_{1}, \\
\mathcal{T}^{-} \lambda & :=\varnothing \lambda_{0}+\sigma \lambda_{1}, & -\mathcal{T}^{+} \lambda & :=\chi^{\prime} \lambda_{1}+\sigma^{\prime} \lambda_{0},
\end{aligned}
$$

where $\lambda:=\left(\lambda_{0}, \lambda_{1}\right)$ and the spinor components are defined by $\lambda_{A}=: \lambda_{1} o_{A}-\lambda_{0} \iota_{A}$. The various first order linear differential operators acting on spinor fields, e.g. the 2-surface twistor operator, the holomorphy/anti-holomorphy operators or the operators whose kernel defines the asymptotic spinors of Bramson [83], are appropriate direct sums of these elementary operators. Their global properties under various circumstances are studied in [43, 356, 363].

\subsection{8 $S O(1,1)$-connection 1-form versus anholonomicity}

Obviously, all the structures we have considered can be introduced on the individual surfaces of one- or two-parameter families of surfaces, too. In particular [181], let the 2 -surface $\mathcal{S}$ be considered as the intersection $\mathcal{N}^{+} \cap \mathcal{N}^{-}$of the null hypersurfaces formed, respectively, by the outgoing and

Living Reviews in Relativity

http: //www. livingreviews . org//rr-2004-4 
the ingoing light rays orthogonal to $\mathcal{S}$, and let the spacetime (or at least a neighbourhood of $\mathcal{S}$ ) be foliated by two one-parameter families of smooth hypersurfaces $\left\{\nu_{+}=\right.$const. $\}$and $\left\{\nu_{-}=\right.$const. $\}$, where $\nu_{ \pm}: M \rightarrow \mathbb{R}$, such that $\mathcal{N}^{+}=\left\{\nu_{+}=0\right\}$ and $\mathcal{N}^{-}=\left\{\nu_{-}=0\right\}$. One can form the two normals, $n_{ \pm a}:=\nabla_{a} \nu_{ \pm}$, which are null on $\mathcal{N}^{+}$and $\mathcal{N}^{-}$, respectively. Then we can define $\beta_{ \pm e}:=\left(\Delta_{e} n_{ \pm a}\right) n_{\mp}^{a}$, for which $\beta_{+e}+\beta_{-e}=\Delta_{e} n^{2}$, where $n^{2}:=g_{a b} n_{+}^{a} n_{-}^{b}$. (If $n^{2}$ is chosen to be 1 on $\mathcal{S}$, then $\beta_{-e}=-\beta_{+e}$ is precisely the $S O(1,1)$ connection 1-form $A_{e}$ above.) Then the so-called anholonomicity is defined by $\omega_{e}:=\frac{1}{2 n^{2}}\left[n_{-}, n_{+}\right]^{f} q_{f e}=\frac{1}{2 n^{2}}\left(\beta_{+e}-\beta_{-e}\right)$. Since $\omega_{e}$ is invariant with respect to the rescalings $\nu_{+} \mapsto \exp (A) \nu_{+}$and $\nu_{-} \mapsto \exp (B) \nu_{-}$of the functions defining the foliations by those functions $A, B: M \rightarrow \mathbb{R}$ which preserve $\nabla_{[a} n_{ \pm b]}=0$, it was claimed in [181] that $\omega_{e}$ depends only on $\mathcal{S}$. However, this implies only that $\omega_{e}$ is invariant with respect to a restricted class of the change of the foliations, and that $\omega_{e}$ is invariantly defined only by this class of the foliations rather than the 2 -surface. In fact, $\omega_{e}$ does depend on the foliation: Starting with a different foliation defined by the functions $\bar{\nu}_{+}:=\exp (\alpha) \nu_{+}$and $\bar{\nu}_{-}:=\exp (\beta) \nu_{-}$for some $\alpha, \beta: M \rightarrow \mathbb{R}$, the corresponding anholonomicity $\bar{\omega}_{e}$ would also be invariant with respect to the restricted changes of the foliations above, but the two anholonomicities, $\omega_{e}$ and $\bar{\omega}_{e}$, would be different: $\bar{\omega}_{e}-\omega_{e}=\frac{1}{2} \Delta_{e}(\alpha-\beta)$. Therefore, the anholonomicity is still a gauge dependent quantity.

\subsection{Standard situations to evaluate the quasi-local quantities}

There are exact solutions to the Einstein equations and classes of special (e.g. asymptotically flat) spacetimes in which there is a commonly accepted definition of energy-momentum (or at least mass) and angular momentum. In this section we review these situations and recall the definition of these 'standard' expressions.

\subsubsection{Round spheres}

If the spacetime is spherically symmetric, then a 2 -sphere which is a transitivity surface of the rotation group is called a round sphere. Then in a spherical coordinate system $(t, r, \theta, \phi)$ the spacetime metric takes the form $g_{a b}=\operatorname{diag}\left(\exp (2 \gamma),-\exp (2 \alpha),-r^{2},-r^{2} \sin ^{2} \theta\right)$, where $\gamma$ and $\alpha$ are functions of $t$ and $r$. (Hence $r$ is the so-called area-coordinate). Then with the notations of Section 4.1, one obtains $R_{a b c d} \varepsilon^{a b} \varepsilon^{c d}=\frac{4}{r^{2}}(1-\exp (-2 \alpha))$. Based on the investigations of Misner, Sharp, and Hernandez [268, 199], Cahill and McVitte [98] found

$$
E(t, r):=\frac{1}{8 G} r^{3} R_{a b c d} \varepsilon^{a b} \varepsilon^{c d}=\frac{r}{2 G}\left(1-e^{-2 \alpha}\right)
$$

to be an appropriate (and hence suggested to be the general) notion of energy contained in the 2-sphere $\mathcal{S}:=\{t=$ const., $r=$ const. $\}$. In particular, for the Reissner-Nordström solution $G E(t, r)=m-e^{2} / 2 r$, while for the isentropic fluid solutions $E(t, r)=4 \pi \int_{0}^{r} r^{\prime 2} \mu\left(t, r^{\prime}\right) d r^{\prime}$, where $m$ and $e$ are the usual parameters of the Reissner-Nordström solutions and $\mu$ is the energy density of the fluid $[268,199]$ (for the static solution, see e.g. Appendix B of [175]). Using Einstein's equations nice and simple equations can be derived for the derivatives $\partial_{t} E(t, r)$ and $\partial_{r} E(t, r)$, and if the energy-momentum tensor satisfies the dominant energy condition then $\partial_{r} E(t, r)>0$. Thus $E(t, r)$ is a monotonic function of $r$ provided $r$ is the area-coordinate. Since by the spherical symmetry all the quantities with non-zero spin weight, in particular the shears $\sigma$ and $\sigma^{\prime}$, are vanishing and $\psi_{2}$ is real, by the GHP form of Equations $(22,23)$ the energy function $E(t, r)$ can also be written as

$$
E(\mathcal{S})=\frac{1}{G} r^{3}\left(\frac{1}{4} \mathcal{S}_{R}+\rho \rho^{\prime}\right)=\frac{1}{G} r^{3}\left(-\psi_{2}+\phi_{11}+\Lambda\right)=\sqrt{\frac{\operatorname{Area}(\mathcal{S})}{16 \pi G^{2}}}\left(1+\frac{1}{2 \pi} \oint_{\mathcal{S}} \rho \rho^{\prime} d \mathcal{S}\right) .
$$


This expression is considered to be the 'standard' definition of the energy for round spheres ${ }^{4}$. Its last expression does not depend on whether $r$ is an area-coordinate or not. $E(\mathcal{S})$ contains a contribution from the gravitational 'field' too. In fact, for example for fluids it is not simply the volume integral of the energy density $\mu$ of the fluid, because that would be $4 \pi \int_{0}^{r} r^{\prime 2} \exp (\alpha) \mu d r^{\prime}$. This deviation can be interpreted as the contribution of the gravitational potential energy to the total energy. Consequently, $E(\mathcal{S})$ is not a globally monotonic function of $r$, even if $\mu \geq 0$. For example, in the closed Friedmann-Robertson-Walker spacetime (where, to cover the whole space, $r$ cannot be chosen to be the area-radius and $r \in[0, \pi]) E(\mathcal{S})$ is increasing for $r \in[0, \pi / 2)$, taking its maximal value at $r=\pi / 2$, and decreasing for $r \in(\pi / 2, \pi]$.

This example suggests a slightly more exotic spherically symmetric spacetime. Its spacelike slice $\Sigma$ will be assumed to be extrinsically flat, and its intrinsic geometry is the matching of two conformally flat metrics. The first is a 'large' spherically symmetric part of a $t=$ const. hypersurface of the closed Friedmann-Robertson-Walker spacetime with the line element $d l^{2}=$ $\Omega_{\mathrm{FRW}}^{2} d l_{0}^{2}$, where $d l_{0}^{2}$ is the line element for the flat 3 -space and $\Omega_{\mathrm{FRW}}^{2}:=B\left(1+\frac{r^{2}}{4 T^{2}}\right)^{-2}$ with some positive constants $B$ and $T^{2}$, and the range of the Euclidean radial coordinate $r$ is $\left[0, r_{0}\right]$, where $r_{0} \in(2 T, \infty)$. It contains a maximal 2-surface at $r=2 T$ with round-sphere mass parameter $M:=G E(2 T)=\frac{1}{2} T \sqrt{B}$. The scalar curvature is $R=6 / B T^{2}$, and hence, by the constraint parts of the Einstein equations and by the vanishing of the extrinsic curvature, the dominant energy condition is satisfied. The other metric is the metric of a piece of a $t=$ const. hypersurface in the Schwarzschild solution with mass parameter $m$ (see [156]): $d l^{2}=\Omega_{S}^{2} d \bar{l}_{0}^{2}$, where $\Omega_{S}^{2}:=\left(1+\frac{m}{2 \bar{r}}\right)^{4}$ and the Euclidean radial coordinate $\bar{r}$ runs from $\bar{r}_{0}$ and $\infty$, where $\bar{r}_{0} \in(0, m / 2)$. In this geometry there is a minimal surface at $\bar{r}=m / 2$, the scalar curvature is zero, and the round sphere energy is $E(\bar{r})=m / G$. These two metrics can be matched to obtain a differentiable metric with Lipschitzcontinuous derivative at the 2-surface of the matching (where the scalar curvature has a jump) with arbitrarily large 'internal mass' $M / G$ and arbitrarily small ADM mass $m / G$. (Obviously, the two metrics can be joined smoothly as well by an 'intermediate' domain between them.) Since this space looks like a big spherical bubble on a nearly flat 3-plane - like the capital Greek letter $\Omega$ for later reference we call it an ' $\Omega_{M, m}$-spacetime'.

Spherically symmetric spacetimes admit a special vector field, the so-called Kodama vector field $K^{a}$, such that $K_{a} G^{a b}$ is divergence free [241]. In asymptotically flat spacetimes $K^{a}$ is timelike in the asymptotic region, in stationary spacetimes it reduces to the Killing symmetry of stationarity (in fact, this is hypersurface-orthogonal), but in general it is not a Killing vector. However, by $\nabla_{a}\left(G^{a b} K_{b}\right)=0$ the vector field $S^{a}:=G^{a b} K_{b}$ has a conserved flux on a spacelike hypersurface $\Sigma$. In particular, in the coordinate system $(t, r, \theta, \phi)$ and line element above $K^{a}=\exp [-(\alpha+\gamma)](\partial / \partial t)^{a}$. If $\Sigma$ is the solid ball of radius $r$, then the flux of $S^{a}$ is precisely the standard round sphere expression (26) for the 2-sphere $\partial \Sigma[278]$.

An interesting characterization of the dynamics of the spherically symmetric gravitational fields can be given in terms of the energy function $E(t, r)$ above (see for example [408, 262, 185]). In particular, criteria for the existence and the formation of trapped surfaces and the presence and the nature of the central singularity can be given by $E(t, r)$.

\subsubsection{Small surfaces}

In the literature there are two notions of small surfaces: The first is that of the small spheres (both in the light cone of a point and in a spacelike hypersurface), introduced first by Horowitz and Schmidt [204], and the other is the concept of the small ellipsoids in some spacelike hypersurface, considered first by Woodhouse in [235]. A small sphere in the light cone is a cut of the future null

\footnotetext{
${ }^{4} E(\mathcal{S})$ can be thought of as the 0-component of some quasi-local energy-momentum 4-vector, but, just because of the spherical symmetry, its spatial parts are vanishing. Thus $E(\mathcal{S})$ can also be interpreted as the mass, the length of this energy-momentum 4-vector.
}

Living Reviews in Relativity

http://www. livingreviews.org//rr-2004-4 
cone in the spacetime by a spacelike hypersurface, and the geometry of the sphere is characterized by data at the vertex of the cone. The sphere in a hypersurface consists of those points of a given spacelike hypersurface, whose geodesic distance in the hypersurface from a given point $p$, the centre, is a small given value, and the geometry of this sphere is characterized by data at this centre. Small ellipsoids are 2-surfaces in a spacelike hypersurface with a more general shape.

To define the first, let $p \in M$ be a point, and $t^{a}$ a future directed unit timelike vector at $p$. Let $\mathcal{N}_{p}:=\partial I^{+}(p)$, the 'future null cone of $p$ in $M^{\prime}$ ' (i.e. the boundary of the chronological future of $p$ ). Let $l^{a}$ be the future pointing null tangent to the null geodesic generators of $\mathcal{N}_{p}$ such that, at the vertex $p, l^{a} t_{a}=1$. With this condition we fix the scale of the affine parameter $r$ on the different generators, and hence by requiring $r(p)=0$ we fix the parameterization completely. Then, in an open neighbourhood of the vertex $p, \mathcal{N}_{p}-\{p\}$ is a smooth null hypersurface, and hence for sufficiently small $r$ the set $\mathcal{S}_{r}:=\{q \in M \mid r(q)=r\}$ is a smooth spacelike 2-surface and homeomorphic to $S^{2}$. $\mathcal{S}_{r}$ is called a small sphere of radius $r$ with vertex $p$. Note that the condition $l^{a} t_{a}=1$ fixes the boost gauge.

Completing $l^{a}$ to a Newman-Penrose complex null tetrad $\left\{l^{a}, n^{a}, m^{a}, \bar{m}^{a}\right\}$ such that the complex null vectors $m^{a}$ and $\bar{m}^{a}$ are tangent to the 2 -surfaces $\mathcal{S}_{r}$, the components of the metric and the spin coefficients with respect to this basis can be expanded as series in $r^{5}$. Then the GHP equations can be solved with any prescribed accuracy for the expansion coefficients of the metric $q_{a b}$ on $\mathcal{S}_{r}$, the GHP spin coefficients $\rho, \sigma, \tau, \rho^{\prime}, \sigma^{\prime}$, and $\beta$, and the higher order expansion coefficients of the curvature in terms of the lower order curvature components at $p$. Hence the expression of any quasi-local quantity $Q_{\mathcal{S}_{r}}$ for the small sphere $\mathcal{S}_{r}$ can be expressed as a series of $r$,

$$
Q_{\mathcal{S}_{r}}=\oint_{\mathcal{S}}\left(Q^{(0)}+r Q^{(1)}+\frac{1}{2} r^{2} Q^{(2)}+\cdots\right) d \mathcal{S}
$$

where the expansion coefficients $Q^{(k)}$ are still functions of the coordinates, $(\zeta, \bar{\zeta})$ or $(\theta, \phi)$, on the unit sphere $\mathcal{S}$. If the quasi-local quantity $Q$ is spacetime-covariant, then the unit sphere integrals of the expansion coefficients $Q^{(k)}$ must be spacetime covariant expressions of the metric and its derivatives up to some finite order at $p$ and the 'time axis' $t^{a}$. The necessary degree of the accuracy of the solution of the GHP equations depends on the nature of $Q_{\mathcal{S}_{r}}$ and on whether the spacetime is Ricci-flat in a neighbourhood of $p$ or not ${ }^{6}$. These solutions of the GHP equations, with increasing accuracy, are given in [204, 235, 94, 360].

Obviously, we can calculate the small sphere limit of various quasi-local quantities built from the matter fields in the Minkowski spacetime, too. In particular [360], the small sphere expressions for the quasi-local energy-momentum and the (anti-self-dual part of the) quasi-local angular momentum of the matter fields based on $Q_{\mathcal{S}}[\mathbf{K}]$, respectively, are

$$
\begin{aligned}
P_{\mathcal{S}_{r}} \underline{B}^{\prime} & =\frac{4 \pi}{3} r^{3} T^{A A^{\prime} B B^{\prime}} t_{A A^{\prime}} \mathcal{E}_{B}^{A} \overline{\mathcal{E}}_{B^{\prime}}^{\underline{B}^{\prime}}+\mathcal{O}\left(r^{4}\right), \\
J_{\mathcal{\mathcal { S }}_{r}} \underline{\underline{B}} & =\frac{4 \pi}{3} r^{3} T_{A A^{\prime} B B^{\prime}} t^{A A^{\prime}}\left(r t^{B^{\prime} E} \varepsilon^{B F} \mathcal{E}_{(E}^{A} \mathcal{E} \frac{B}{F)}\right)+\mathcal{O}\left(r^{5}\right),
\end{aligned}
$$

where $\left\{\mathcal{E}_{\bar{A}}^{A}\right\}, \underline{A}=0,1$, is the 'Cartesian spin frame' at $p$ and the origin of the Cartesian coordinate system is chosen to be the vertex $p$. Here $K_{a}^{A} \underline{B}^{\prime}=\mathcal{E}_{A}^{A} \overline{\mathcal{E}}_{\bar{A}^{\prime}}^{\underline{B^{\prime}}}$ can be interpreted as the translation 1-forms, while $K_{a}^{A} \underline{B}=r t_{A^{\prime}}{ }^{E} \mathcal{E}_{(E}^{A} \mathcal{E}_{A)}^{B}$ is an average on the unit sphere of the boost-rotation Killing 1 -forms that vanish at the vertex $p$. Thus $P_{\mathcal{S}_{r}}^{\underline{A}} \underline{B}^{\prime}$ and $J_{\mathcal{S}_{r}}^{\underline{B}}$ are the 3 -volume times the energymomentum and angular momentum density with respect to $p$, respectively, that the observer with 4 -velocity $t^{a}$ sees at $p$.

\footnotetext{
${ }^{5}$ If, in addition, the spinor constituent $o^{A}$ of $l^{a}=o^{A} \bar{o}^{A^{\prime}}$ is required to be parallel propagated along $l^{a}$, then the tetrad becomes completely fixed, yielding the vanishing of several (combinations of the) spin coefficients.

${ }^{6}$ As we will see soon, the leading term of the small sphere expression of the energy-momenta in non-vacuum is of order $r^{3}$, in vacuum it is $r^{5}$, while that of the angular momentum is $r^{4}$ and $r^{6}$, respectively.
} 
Interestingly enough, a simple dimensional analysis already shows the structure of the leading terms in a large class of quasi-local spacetime covariant energy-momentum and angular momentum expressions. In fact, if $Q_{\mathcal{S}}$ is any coordinate-independent quasi-local quantity, built from the first derivatives of the metric, i.e. $Q_{\mathcal{S}}=\oint_{\mathcal{S}}\left(\partial_{\mu} g_{\alpha \beta}\right) d \mathcal{S}$, then its expansion is

$$
\begin{aligned}
Q_{\mathcal{S}_{r}}= & {[\partial g] r^{2}+\left[\partial^{2} g,(\partial g)^{2}\right] r^{3}+\left[\partial^{3} g,\left(\partial^{2} g\right)(\partial g),(\partial g)^{3}\right] r^{4}+} \\
& +\left[\partial^{4} g,\left(\partial^{3} g\right)(\partial g),\left(\partial^{2} g\right)^{2},\left(\partial^{2} g\right)(\partial g)^{2},(\partial g)^{4}\right] r^{5}+\ldots
\end{aligned}
$$

where $[A, B, \ldots]$ is a scalar. It depends linearly on the tensors constructed from $g_{\alpha \beta}$ and linearly from the coordinate dependent quantities $A, B, \ldots$, and it is a polynomial expression of $t^{a}, g_{a b}$ and $\varepsilon_{a b c d}$ at the vertex $p$. Since there is no non-trivial tensor built from the first derivative $\partial_{\mu} g_{\alpha \beta}$ and $g_{\alpha \beta}$, the leading term is of order $r^{3}$. Its coefficient $\left[\partial^{2} g,(\partial g)^{2}\right]$ must be a linear expression of $R_{a b}$ and $C_{a b c d}$, and polynomial in $t^{a}, g_{a b}$ and $\varepsilon_{a b c d}$. In particular, if $Q_{\mathcal{S}}$ is to represent energymomentum with generator $K^{c}$ at $p$, then the leading term must be

$$
Q_{\mathcal{S}_{r}}[\mathbf{K}]=r^{3}\left[a\left(G_{a b} t^{a} t^{b}\right) t_{c}+b R t_{c}+c\left(G_{a b} t^{a} P_{c}^{b}\right)\right] K^{c}+\mathcal{O}\left(r^{4}\right)
$$

for some unspecified constants $a, b$, and $c$, where $P_{b}^{a}:=\delta_{b}^{a}-t^{a} t_{b}$, the projection to the subspace orthogonal to $t^{a}$. If, in addition to the coordinate-independence of $Q_{\mathcal{S}}$, it is Lorentz-covariant, i.e. for example it does not depend on the choice for a normal to $\mathcal{S}$ (e.g. in the small sphere approximation on $t^{a}$ ) intrinsically, then the different terms in the above expression must depend on the boost gauge of the external observer $t^{a}$ in the same way. Therefore, $a=c$, whenever the first and the third terms can in fact be written as $r^{3} a t^{a} G_{a b} K^{b}$. Then, comparing Equation (30) with Equation (28), we see that $a=-1 /(6 G)$, and then the term $r^{3} b R t_{a} K^{a}$ would have to be interpreted as the contribution of the gravitational 'field' to the quasi-local energy-momentum of the matter + gravity system. However, this contributes only to energy but not to linear momentum in any frame defined by the observer $t^{a}$, even in a general spacetime. This seems to be quite unreasonable. Furthermore, even if the matter fields satisfy the dominant energy condition, $Q_{\mathcal{S}_{r}}$ given by Equation (30) can be negative even for $c=a$ unless $b=0$. Thus, in the leading $r^{3}$ order in non-vacuum any coordinate and Lorentz-covariant quasi-local energy-momentum expression which is non-spacelike and future pointing should be proportional to the energy-momentum density of the matter fields seen by the observer $t^{a}$ times the Euclidean volume of the 3-ball of radius $r$.

If a neighbourhood of $p$ is vacuum, then the $r^{3}$ order term is vanishing, and the fourth order term must be built from $\nabla_{e} C_{a b c d}$. However, the only scalar polynomial expression of $t^{a}, g_{a b}, \varepsilon_{a b c d}$, $\nabla_{e} C_{a b c d}$ and the generator vector $K^{a}$, depending on the latter two linearly, is the zero. Thus the $r^{4}$ order term in vacuum is also vanishing. In the fifth order the only non-zero terms are quadratic in the various parts of the Weyl tensor, yielding

$$
Q_{\mathcal{S}_{r}}[\mathbf{K}]=r^{5}\left[\left(a E_{a b} E^{a b}+b H_{a b} H^{a b}+c E_{a b} H^{a b}\right) t_{c}+d E_{a e} H^{e}{ }_{b} \varepsilon^{a b}{ }_{c}\right] K^{c}+\mathcal{O}\left(r^{6}\right)
$$

for some constants $a, b, c$, and $d$, where $E_{a b}:=C_{a e b f} t^{e} t^{f}$ is the electric and $H_{a b}:=* C_{a e b f} t^{e} t^{f}:=$ $\frac{1}{2} \varepsilon_{a e}{ }^{c d} C_{c d b f} t^{e} t^{f}$ is the magnetic part of the Weyl curvature, and $\varepsilon_{a b c}:=\varepsilon_{a b c d} t^{d}$ is the induced volume 3 -form. However, using the identities $C_{a b c d} C^{a b c d}=8\left(E_{a b} E^{a b}-H_{a b} H^{a b}\right), C_{a b c d} * C^{a b c d}=$ $16 E_{a b} H^{a b}, 4 T_{a b c d} t^{a} t^{b} t^{c} t^{d}=E_{a b} E^{a b}+H_{a b} H^{a b}$ and $2 T_{a b c d} t^{a} t^{b} t^{c} P_{e}^{d}=E_{a b} H^{a}{ }_{c} \varepsilon^{b c}{ }_{e}$, we can rewrite the above formula to

$$
\begin{aligned}
Q_{\mathcal{S}_{r}}[\mathbf{K}]=r^{5}[ & \left(2(a+b) T_{a b c d} t^{a} t^{b} t^{c} t^{d}+\frac{1}{16}(a-b) C_{a b c d} C^{a b c d}+\right. \\
& \left.\left.+\frac{1}{16} c C_{a b c d} * C^{a b c d}\right) t_{e}+2 d T_{a b c d} t^{a} t^{b} t^{c} P_{e}^{d}\right] K^{e}+\mathcal{O}\left(r^{6}\right) .
\end{aligned}
$$

Again, if $Q_{\mathcal{S}}$ does not depend on $t^{a}$ intrinsically, then $d=(a+b)$, whenever the first and the fourth terms together can be written into the Lorentz covariant form $2 r^{5} d T_{a b c d} t^{a} t^{b} t^{c} K^{d}$. In a

Living Reviews in Relativity

http://www. livingreviews.org//rr-2004-4 
general expression the curvature invariants $C_{a b c d} C^{a b c d}$ and $C_{a b c d} * C^{a b c d}$ may be present. Since, however, $E_{a b}$ and $H_{a b}$ at a given point are independent, these invariants can be arbitrarily large positive or negative, and hence for $a \neq b$ or $c \neq 0$ the quasi-local energy-momentum could not be future pointing and non-spacelike. Therefore, in vacuum in the leading $r^{5}$ order any coordinate and Lorentz-covariant quasi-local energy-momentum expression which is non-spacelike and future pointing must be proportional to the Bel-Robinson 'momentum' $T_{a b c d} t^{a} t^{b} t^{c}$.

Obviously, the same analysis can be repeated for any other quasi-local quantity. For quasi-local angular momentum $Q_{\mathcal{S}}$ has the structure $\oint_{\mathcal{S}}\left(\partial_{\mu} g_{\alpha \beta}\right) r d \mathcal{S}$, while the area of $\mathcal{S}$ is $\oint_{\mathcal{S}} d \mathcal{S}$. Then the leading term in the expansion of the angular momentum is $r^{4}$ and $r^{6}$ order in non-vacuum and vacuum, respectively, while the first non-trivial correction to the area $4 \pi r^{2}$ is of order $r^{4}$ and $r^{6}$ in non-vacuum and vacuum, respectively.

On the small geodesic sphere $\mathcal{S}_{r}$ of radius $r$ in the given spacelike hypersurface $\Sigma$ one can introduce the complex null tangents $m^{a}$ and $\bar{m}^{a}$ above, and if $t^{a}$ is the future pointing unit normal of $\Sigma$ and $v^{a}$ the outward directed unit normal of $\mathcal{S}_{r}$ in $\Sigma$, then we can define $l^{a}:=t^{a}+v^{a}$ and $2 n^{a}:=t^{a}-v^{a}$. Then $\left\{l^{a}, n^{a}, m^{a}, \bar{m}^{a}\right\}$ is a Newman-Penrose complex null tetrad, and the relevant GHP equations can be solved for the spin coefficients in terms of the curvature components at $p$.

The small ellipsoids are defined as follows [235]. If $f$ is any smooth function on $\Sigma$ with a non-degenerate minimum at $p \in \Sigma$ with minimum value $f(p)=0$, then, at least on an open neighbourhood $U$ of $p$ in $\Sigma$ the level surfaces $\mathcal{S}_{r}:=\left\{q \in \Sigma \mid 2 f(q)=r^{2}\right\}$ are smooth compact 2 -surfaces homeomorphic to $S^{2}$. Then, in the $r \rightarrow 0$ limit, the surfaces $\mathcal{S}_{r}$ look like small nested ellipsoids centred in $p$. The function $f$ is usually 'normalized' so that $\left.h^{a b} D_{a} D_{b} f\right|_{p}=-3$.

\subsubsection{Large spheres near the spatial infinity}

Near spatial infinity we have the a priori $1 / r$ and $1 / r^{2}$ fall-off for the 3 -metric $h_{a b}$ and extrinsic curvature $\chi_{a b}$, respectively, and both the evolution equations of general relativity and the conservation equation $T_{; b}^{a b}=0$ for the matter fields preserve these conditions. The spheres $\mathcal{S}_{r}$ of coordinate radius $r$ in $\Sigma$ are called large spheres if the values of $r$ are large enough such that the asymptotic expansions of the metric and extrinsic curvature are legitimate ${ }^{7}$. Introducing some coordinate system, e.g. the complex stereographic coordinates, on one sphere and then extending that to the whole $\Sigma$ along the normals $v^{a}$ of the spheres, we obtain a coordinate system $(r, \zeta, \bar{\zeta})$ on $\Sigma$. Let $\varepsilon_{\mathbf{A}}^{A}=\left\{o^{A}, \iota^{A}\right\}, \mathbf{A}=0,1$, be a GHP spinor dyad on $\Sigma$ adapted to the large spheres in such a way that $m^{a}:=o^{A} \bar{\iota}^{A^{\prime}}$ and $\bar{m}^{a}=\iota^{A} \bar{o}^{A^{\prime}}$ are tangent to the spheres and $t^{a}=\frac{1}{2} o^{A} \bar{o}^{A^{\prime}}+\iota^{A} \bar{\iota}^{A^{\prime}}$, the future directed unit normal of $\Sigma$. These conditions fix the spinor dyad completely, and, in particular, $v^{a}=\frac{1}{2} o^{A} \bar{o}^{A^{\prime}}-\iota^{A} \bar{\iota}^{A^{\prime}}$, the outward directed unit normal to the spheres tangent to $\Sigma$.

The fall-off conditions yield that the spin coefficients tend to their flat spacetime value like $1 / r^{2}$ and the curvature components to zero like $1 / r^{3}$. Expanding the spin coefficients and curvature components as power series of $1 / r$, one can solve the field equations asymptotically (see $[48,44]$ for a different formalism). However, in most calculations of the large sphere limit of the quasi-local quantities only the leading terms of the spin coefficients and curvature components appear. Thus it is not necessary to solve the field equations for their second or higher order non-trivial expansion coefficients.

Using the flat background metric ${ }_{0} h_{a b}$ and the corresponding derivative operator ${ }_{0} D_{e}$ we can define a spinor field ${ }_{0} \lambda_{A}$ to be constant if ${ }_{0} D_{e 0} \lambda_{A}=0$. Obviously, the constant spinors form a two complex dimensional vector space. Then by the fall-off properties $D_{e 0} \lambda_{A}=\mathcal{O}\left(r^{-2}\right)$. Hence we can define the asymptotically constant spinor fields to be those $\lambda_{A}$ that satisfy $D_{e} \lambda_{A}=\mathcal{O}\left(r^{-2}\right)$, where $D_{e}$ is the intrinsic Levi-Civita derivative operator. Note that this implies that, with the notations

\footnotetext{
${ }^{7}$ Because of the fall-off, no essential ambiguity in the definition of the large spheres arises from the use of the coordinate radius instead of the physical radial distance.
} 
of Equation (25), all the chiral irreducible parts, $\Delta^{+} \lambda, \Delta^{-} \lambda, \mathcal{T}^{+} \lambda$, and $\mathcal{T}^{-} \lambda$, of the derivative of the asymptotically constant spinor field $\lambda_{A}$ are $\mathcal{O}\left(r^{-2}\right)$.

\subsubsection{Large spheres near null infinity}

Let the spacetime be asymptotically flat at future null infinity in the sense of Penrose [300, 301, 302, 313] (see also [151]), i.e. the physical spacetime can be conformally compactified by an appropriate boundary $\mathscr{I}^{+}$. Then future null infinity $\mathscr{I}^{+}$will be a null hypersurface in the conformally rescaled spacetime. Topologically it is $\mathbb{R} \times S^{2}$, and the conformal factor can always be chosen such that the induced metric on the compact spacelike slices of $\mathscr{I}^{+}$is the metric of the unit sphere. Fixing such a slice $\mathcal{S}_{0}$ (called 'the origin cut of $\mathscr{I}^{+}$) the points of $\mathscr{I}^{+}$can be labeled by a null coordinate, namely the affine parameter $u \in \mathbb{R}$ along the null geodesic generators of $\mathscr{I}^{+}$measured from $\mathcal{S}_{0}$ and, for example, the familiar complex stereographic coordinates $(\zeta, \bar{\zeta}) \in S^{2}$, defined first on the unit sphere $\mathcal{S}_{0}$ and then extended in a natural way along the null generators to the whole $\mathscr{I}^{+}$. Then any other cut $\mathcal{S}$ of $\mathscr{I}^{+}$can be specified by a function $u=f(\zeta, \bar{\zeta})$. In particular, the cuts $\mathcal{S}_{u}:=\{u=$ const. $\}$ are obtained from $\mathcal{S}_{0}$ by a pure time translation.

The coordinates $(u, \zeta, \bar{\zeta})$ can be extended to an open neighbourhood of $\mathscr{I}^{+}$in the spacetime in the following way. Let $\mathcal{N}_{u}$ be the family of smooth outgoing null hypersurfaces in a neighbourhood of $\mathscr{I}^{+}$such that they intersect the null infinity just in the cuts $\mathcal{S}_{u}$, i.e. $\mathcal{N}_{u} \cap \mathscr{I}^{+}=\mathcal{S}_{u}$. Then let $r$ be the affine parameter in the physical metric along the null geodesic generators of $\mathcal{N}_{u}$. Then $(u, r, \zeta, \bar{\zeta})$ forms a coordinate system. The $u=$ const., $r=$ const. 2-surfaces $\mathcal{S}_{u, r}$ (or simply $\mathcal{S}_{r}$ if no confusion can arise) are spacelike topological 2-spheres, which are called large spheres of radius $r$ near future null infinity. Obviously, the affine parameter $r$ is not unique, its origin can be changed freely: $\bar{r}:=r+g(u, \zeta, \bar{\zeta})$ is an equally good affine parameter for any smooth $g$. Imposing certain additional conditions to rule out such coordinate ambiguities we arrive at a 'Bondi-type coordinate system $^{8}$. In many of the large sphere calculations of the quasi-local quantities the large spheres should be assumed to be large spheres not only in a general null, but in a Bondi-type coordinate system. For the detailed discussion of the coordinate freedom left at the various stages in the introduction of these coordinate systems, see for example [290, 289, 84].

In addition to the coordinate system we need a Newman-Penrose null tetrad, or rather a GHP spinor dyad, $\varepsilon_{\mathbf{A}}^{A}=\left\{o^{A}, \iota^{A}\right\}, \mathbf{A}=0,1$, on the hypersurfaces $\mathcal{N}_{u}$. (Thus boldface indices are referring to the GHP spin frame.) It is natural to choose $o^{A}$ such that $l^{a}:=o^{A} \bar{o}^{A^{\prime}}$ be the tangent $(\partial / \partial r)^{a}$ of the null geodesic generators of $\mathcal{N}_{u}$, and $o^{A}$ itself be constant along $l^{a}$. Newman and Unti [290] chose $\iota^{A}$ to be parallel propagated along $l^{a}$. This choice yields the vanishing of a number of spin coefficients (see for example the review [289]). The asymptotic solution of the Einstein-Maxwell equations as a series of $1 / r$ in this coordinate and tetrad system is given in [290,134,312], where all the non-vanishing spin coefficients and metric and curvature components are listed. In this formalism the gravitational waves are represented by the $u$-derivative $\dot{\sigma}^{0}$ of the asymptotic shear of the null geodesic generators of the outgoing null hypersurfaces $\mathcal{N}_{u}$.

From the point of view of the large sphere calculations of the quasi-local quantities the choice of Newman and Unti for the spinor basis is not very convenient. It is more natural to adapt the GHP spin frame to the family of the large spheres of constant 'radius' $r$, i.e. to require $m^{a}:=o^{A} \bar{\iota}^{A^{\prime}}$ and $\bar{m}^{a}=\iota^{A} \bar{o}^{A^{\prime}}$ to be tangents of the spheres. This can be achieved by an appropriate null rotation of the Newman-Unti basis about the spinor $o^{A}$. This rotation yields a change of the spin coefficients and the metric and curvature components. As far as the present author is aware of, this rotation with the highest accuracy was done for the solutions of the Einstein-Maxwell system by Shaw [338].

In contrast to the spatial infinity case, the 'natural' definition of the asymptotically constant spinor fields yields identically zero spinors in general [83]. Nontrivial constant spinors in this

\footnotetext{
${ }^{8}$ In the so-called Bondi coordinate system the radial coordinate is the luminosity distance $r_{\mathrm{D}}:=-1 / \rho$, which tends to the affine parameter $r$ asymptotically.
}

Living Reviews in Relativity

http://www. livingreviews.org//rr-2004-4 
sense could exist only in the absence of the outgoing gravitational radiation, i.e. when $\dot{\sigma}^{0}=0$. In the language of Section 4.1.7, this definition would be $\lim _{r \rightarrow \infty} r \Delta^{+} \lambda=0, \lim _{r \rightarrow \infty} r \Delta^{-} \lambda=0$, $\lim _{r \rightarrow \infty} r \mathcal{T}^{+} \lambda=0$ and $\lim _{r \rightarrow \infty} r \mathcal{T}^{-} \lambda=0$. However, as Bramson showed [83], half of these conditions can be imposed. Namely, at future null infinity $\mathcal{C}^{+} \lambda:=\left(\Delta^{+} \oplus \mathcal{T}^{-}\right) \lambda=0$ (and at past null infinity $\left.\mathcal{C}^{-} \lambda:=\left(\Delta^{-} \oplus \mathcal{T}^{+}\right) \lambda=0\right)$ can always be imposed asymptotically, and it has two linearly independent solutions $\lambda_{A}^{A}, \underline{A}=0,1$, on $\mathscr{I}^{+}$(or on $\mathscr{I}^{-}$, respectively). The space $\mathbf{S}_{\infty}^{A}$ of its solutions turns out to have a natural symplectic metric $\varepsilon_{\underline{A}} \underline{B}$, and we refer to $\left(\mathbf{S}_{\infty}^{A}, \varepsilon_{\underline{A}} \underline{B}\right)$ as future asymptotic spin space. Its elements are called asymptotic spinors, and the equations $\lim _{r \rightarrow \infty} r \mathcal{C}^{ \pm} \lambda=0$ the future/past asymptotic twistor equations. At $\mathscr{I}^{+}$asymptotic spinors are the spinor constituents of the BMS translations: Any such translation is of the form $K^{A} \underline{A}^{\prime} \lambda_{\underline{A}}^{A} \bar{\lambda}_{\underline{A}^{\prime}}^{A^{\prime}}=$

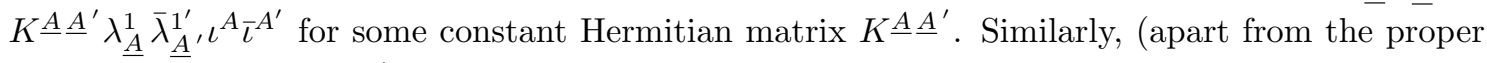
supertranslation content) the components of the anti-self-dual part of the boost-rotation BMS vector fields are $-\sigma_{\mathbf{i}}^{A} \underline{B} \lambda_{\underline{A}}^{1} \lambda_{\underline{B}}^{1}$, where $\sigma_{\dot{\mathbf{i}}}^{\underline{A}} \underline{B}$ are the standard $S U(2)$ Pauli matrices (divided by $\sqrt{2}$ ) [363]. Asymptotic spinors can be recovered as the elements of the kernel of several other operators built from $\Delta^{+}, \Delta^{-}, \mathcal{T}^{+}$, and $\mathcal{T}^{-}$, too. In the present review we use only the fact that asymptotic spinors can be introduced as anti-holomorphic spinors (see also Section 8.2.1), i.e. the solutions of $\mathcal{H}^{-} \lambda:=\left(\Delta^{-} \oplus \mathcal{T}^{-}\right) \lambda=0$ (and at past null infinity as holomorphic spinors), and as special solutions of the 2 -surface twistor equation $\mathcal{T} \lambda:=\left(\mathcal{T}^{+} \oplus \mathcal{T}^{-}\right) \lambda=0$ (see also Section 7.2.1). These operators, together with others reproducing the asymptotic spinors, are discussed in [363].

The Bondi-Sachs energy-momentum given in the Newman-Penrose formalism has already become its 'standard' form. It is the unit sphere integral on the cut $\mathcal{S}$ of a combination of the leading term $\psi_{2}^{0}$ of the Weyl spinor component $\psi_{2}$, the asymptotic shear $\sigma^{0}$ and its $u$-derivative, weighted by the first four spherical harmonics (see for example [289, 313]):

$$
P_{\bar{B}}^{\frac{A}{B^{\prime}}}{ }^{\prime}=-\frac{1}{4 \pi G} \oint\left(\psi_{2}^{0}+\sigma^{0} \dot{\bar{\sigma}}^{0}\right) \lambda_{0}^{A} \bar{\lambda}_{0^{\prime}}{ }^{\prime} d \mathcal{S},
$$

where $\lambda_{0}^{A}:=\lambda \frac{A}{A} o^{A}, \underline{A}=0,1$, are the $o^{A}$-component of the vectors of a spin frame in the space of the asymptotic spinors. (For the various realizations of these spinors see for example [363].)

Similarly, the various definitions for angular momentum at null infinity could be rewritten in this formalism. Although there is no generally accepted definition for angular momentum at null infinity in general spacetimes, in stationary spacetimes there is. It is the unit sphere integral on the cut $\mathcal{S}$ of the leading term of the Weyl spinor component $\bar{\psi}_{1^{\prime}}$, weighted by appropriate (spin weighted) spherical harmonics:

$$
J^{\underline{A}} \underline{B}=\frac{1}{8 \pi G} \oint \bar{\psi}_{1^{\prime}}^{0} \lambda_{0}^{\underline{A}} \lambda_{0}^{\underline{B}} d \mathcal{S} .
$$

In particular, Bramson's expression also reduces to this 'standard' expression in the absence of the outgoing gravitational radiation [86].

\subsubsection{Other special situations}

In the weak field approximation of general relativity [382, 22, 387, 313, 227] the gravitational field is described by a symmetric tensor field $h_{a b}$ on Minkowski spacetime $\left(\mathbb{R}^{4}, g_{a b}^{0}\right)$, and the dynamics of the field $h_{a b}$ is governed by the linearized Einstein equations, i.e. essentially the wave equation. Therefore, the tools and techniques of the Poincaré-invariant field theories, in particular the Noether-Belinfante-Rosenfeld procedure outlined in Section 2.1 and the ten Killing vectors of the background Minkowski spacetime, can be used to construct the conserved quantities. It turns out that the symmetric energy-momentum tensor of the field $h_{a b}$ is essentially the second order term in the Einstein tensor of the metric $g_{a b}:=g_{a b}^{0}+h_{a b}$. Thus in the linear approximation the field $h_{a b}$ 
does not contribute to the global energy-momentum and angular momentum of the matter + gravity system, and hence these quantities have the form (5) with the linearized energy-momentum tensor of the matter fields. However, as we will see in Section 7.1.1, this energy-momentum and angular momentum can be re-expressed as a charge integral of the (linearized) curvature [349, 206, 313].

$p p$-waves spacetimes are defined to be those that admit a constant null vector field $L^{a}$, and they are interpreted as describing pure plane-fronted gravitational waves with parallel rays. If matter is present then it is necessarily pure radiation with wavevector $L^{a}$, i.e. $T^{a b} L_{b}=0$ holds [243]. A remarkable feature of the $p p$-wave metrics is that, in the usual coordinate system, the Einstein equations become a two dimensional linear equation for a single function. In contrast to the approach adopted almost exclusively, Aichelburg [3] considered this field equation as an equation for a boundary value problem. As we will see, from the point of view of the quasi-local observables this is a particularly useful and natural standpoint. If a $p p$-wave spacetime admits an additional spacelike Killing vector $K^{a}$ with closed $S^{1}$ orbits, i.e. it is cyclically symmetric too, then $L^{a}$ and $K^{a}$ are necessarily commuting and are orthogonal to each other, because otherwise an additional timelike Killing vector would also be admitted [351].

Since the final state of stellar evolution (the neutron star or the black hole state) is expected to be described by an asymptotically flat stationary, axi-symmetric spacetime, the significance of these spacetimes is obvious. It is conjectured that this final state is described by the KerrNewman (either outer or black hole) solution with some well-defined mass, angular momentum and electric charge parameters [387]. Thus axi-symmetric 2-surfaces in these solutions may provide domains which are general enough but for which the quasi-local quantities are still computable. According to a conjecture by Penrose [305], the (square root of the) area of the event horizon provides a lower bound for the total ADM energy. For the Kerr-Newman black hole this area is $4 \pi\left(2 m^{2}-e^{2}+2 m \sqrt{m^{2}-e^{2}-a^{2}}\right)$. Thus, particularly interesting 2-surfaces in these spacetimes are the spacelike cross sections of the event horizon [62].

There is a well-defined notion of total energy-momentum not only in the asymptotically flat, but even in the asymptotically anti-de-Sitter spacetimes too. This is the Abbott-Deser energy [1], whose positivity has also been proven under similar conditions that we had to impose in the positivity proof of the ADM energy [161]. (In the presence of matter fields, e.g. a self-interacting scalar field, the fall-off properties of the metric can be weakened such that the 'charges' defined at infinity and corresponding to the asymptotic symmetry generators remain finite [198].) The conformal technique, initiated by Penrose, is used to give a precise definition of the asymptotically anti-de-Sitter spacetimes and to study their general, basic properties in [27]. A comparison and analysis of the various definitions of mass for asymptotically anti-de-Sitter metrics is given in [117]. Thus it is natural to ask whether a specific quasi-local energy-momentum expression is able to reproduce the Abbott-Deser energy-momentum in this limit or not.

\subsection{On lists of criteria of reasonableness of the quasi-local quantities}

In the literature there are various, more or less ad hoc, 'lists of criteria of reasonableness' of the quasi-local quantities (see for example [131, 111]). However, before discussing them, it seems useful to formulate first some general principles that any quasi-local quantity should satisfy.

\subsubsection{General expectations}

In non-gravitational physics the notions of conserved quantities are connected with symmetries of the system, and they are introduced through some systematic procedure in the Lagrangian and/or Hamiltonian formalism. In general relativity the total energy-momentum and angular momentum are 2-surface observables, thus we concentrate on them even at the quasi-local level. These facts motivate our three a priori expectations:

Living Reviews in Relativity

http: //www. livingreviews . org//rr-2004-4 
1. The quasi-local quantities that are 2-surface observables should depend only on the 2-surface data, but they cannot depend e.g. on the way that the various geometric structures on $\mathcal{S}$ are extended off the 2 -surface. There seems to be no a priori reason why the 2 -surface would have to be restricted to have spherical topology. Thus, in the ideal case, the general construction of the quasi-local energy-momentum and angular momentum should work for any closed orientable spacelike 2 -surface.

2. It is desirable to derive the quasi-local energy-momentum and angular momentum as the charge integral (Lagrangian interpretation) and/or as the value of the Hamiltonian on the constraint surface in the phase space (Hamiltonian interpretation). If they are introduced in some other way, they should have a Lagrangian and/or Hamiltonian interpretation.

3. These quantities should correspond to the 'quasi-symmetries' of the 2-surface. In particular, the quasi-local energy-momentum should be expected to be in the dual of the space of the 'quasi-translations', and the angular momentum in the dual of the space of the 'quasirotations'.

To see that these conditions are non-trivial, let us consider the expressions based on the linkage integral (16). $L_{\mathcal{S}}[\mathbf{K}]$ does not satisfy the first part of Requirement 1 . In fact, it depends on the derivative of the normal components of $K^{a}$ in the direction orthogonal to $\mathcal{S}$ for any value of the parameter $\alpha$. Thus it depends not only on the geometry of $\mathcal{S}$ and the vector field $K^{a}$ given on the 2-surface, but on the way in which $K^{a}$ is extended off the 2-surface. Therefore, $L_{\mathcal{S}}[\mathbf{K}]$ is 'less quasi-local' than $A_{\mathcal{S}}[\omega]$ or $H_{\mathcal{S}}[\lambda, \bar{\mu}]$ introduced in Sections 7.2.1 and 7.2.2, respectively.

We will see that the Hawking energy satisfies Requirement 1, but not Requirements 2 and 3. The Komar integral (i.e. the linkage for $\alpha=0$ ) has the form of the charge integral of a superpotential: $8 \pi G K_{\mathcal{S}}[\mathbf{K}]:=\oint_{\mathcal{S}} \nabla^{[a} K^{b]} \frac{1}{2} \varepsilon_{a b c d}$, i.e. it has a Lagrangian interpretation. The corresponding conserved Komar-current is defined by $8 \pi G_{K} C^{a}[\mathbf{K}]:=G^{a}{ }_{b} K^{b}+\nabla_{b} \nabla^{[a} K^{b]}$. However, its flux integral on some compact spacelike hypersurface with boundary $\mathcal{S}:=\partial \Sigma$ cannot be a Hamiltonian on the ADM phase space in general. In fact, it is

$$
\begin{aligned}
{ }_{K} H[\mathbf{K}] & :=\int_{\Sigma}{ }_{K} C^{a}[\mathbf{K}] t_{a} d \Sigma= \\
& =\int_{\Sigma}\left(c N+c_{a} N^{a}\right) d \Sigma+\frac{1}{8 \pi G} \oint_{\mathcal{S}} v_{a}\left(\chi^{a}{ }_{b} N^{b}-D^{a} N+\frac{1}{2 N} \dot{N}^{a}\right) d \mathcal{S} .
\end{aligned}
$$

Here $c$ and $c_{a}$ are, respectively, the Hamiltonian and momentum constraints of the vacuum theory, $t^{a}$ is the future directed unit normal to $\Sigma, v^{a}$ is the outward directed unit normal to $\mathcal{S}$ in $\Sigma$, and $N$ and $N^{a}$ are the lapse and shift part of $K^{a}$, respectively, defined by $K^{a}=: N t^{a}+N^{a}$. Thus ${ }_{K} H[\mathbf{K}]$ is a well-defined function of the configuration and velocity variables $\left(N, N^{a}, h_{a b}\right)$ and $\left(\dot{N}, \dot{N}^{a}, \dot{h}_{a b}\right)$, respectively. However, since the velocity $\dot{N}^{a}$ cannot be expressed by the canonical variables $[396,46],{ }_{K} H[\mathbf{K}]$ can be written as a function on the ADM phase space only if the boundary conditions at $\partial \Sigma$ ensure the vanishing of the integral of $v_{a} \dot{N}^{a} / N$.

\subsubsection{Pragmatic criteria}

Since in certain special situations there are generally accepted definitions for the energy-momentum and angular momentum, it seems reasonable to expect that in these situations the quasi-local quantities reduce to them. One half of the pragmatic criteria is just this expectation, and the other is a list of some a priori requirements on the behaviour of the quasi-local quantities.

One such list for the energy-momentum and mass, based mostly on $[131,111]$ and the properties of the quasi-local energy-momentum of the matter fields of Section 2.2, might be the following: 
1.1 The quasi-local energy-momentum $P_{\mathcal{\mathcal { S }}}^{\underline{a}}$ must be a future pointing nonspacelike vector (assuming that the matter fields satisfy the dominant energy condition on some $\Sigma$ for which $\mathcal{S}=\partial \Sigma$, and maybe some form of the convexity of $\mathcal{S}$ should be required) ('positivity').

$1.2 P \frac{a}{\mathcal{S}}$ must be zero iff $D(\Sigma)$ is flat, and null iff $D(\Sigma)$ has a $p p$-wave geometry with pure radiation ('rigidity').

$1.3 P_{\mathcal{S}}^{\underline{a}}$ must give the correct weak field limit.

$1.4 P_{\mathcal{S}}^{\underline{a}}$ must reproduce the ADM, Bondi-Sachs and Abbott-Deser energy-momenta in the appropriate limits ('correct large sphere behaviour').

1.5 For small spheres $P_{\mathcal{S}}^{\underline{a}}$ must give the expected results ('correct small sphere behaviour'):

1. $\frac{4}{3} \pi r^{3} T^{a b} t_{b}$ in non-vacuum and

2. $k r^{5} T^{a b c d} t_{b} t_{c} t_{d}$ in vacuum for some positive constant $k$ and the Bel-Robinson tensor $T^{a b c d}$

1.6 For round spheres $P_{\mathcal{S}}^{\underline{a}}$ must yield the 'standard' round sphere expression.

1.7 For marginally trapped surfaces the quasi-local mass $m_{\mathcal{S}}$ must be the irreducible mass $\sqrt{\operatorname{Area}(\mathcal{S}) / 16 \pi G^{2}}$.

Item 1.7 is motivated by the expectation that the quasi-local mass associated with the apparent horizon of a black hole (i.e. the outermost marginally trapped surface in a spacelike slice) be just the irreducible mass $[131,111]$. Usually, $m_{\mathcal{S}}$ is expected to be monotonic in some appropriate sense [111]. For example, if $\mathcal{S}_{1}=\partial \Sigma$ for some achronal (and hence spacelike or null) hypersurface $\Sigma$ in which $\mathcal{S}_{2}$ is a spacelike closed 2-surface and the dominant energy condition is satisfied on $\Sigma$, then $m_{\mathcal{S}_{1}} \geq m_{\mathcal{S}_{2}}$ seems to be a reasonable expectation [131]. (But see also the next Section 4.3.3.) On the other hand, in contrast to the energy-momentum and angular momentum of the matter fields on the Minkowski spacetime, the additivity of the energy-momentum (and angular momentum) is not expected. In fact, if $\mathcal{S}_{1}$ and $\mathcal{S}_{2}$ are two connected 2-surfaces, then, for example, the corresponding quasi-local energy-momenta would belong to different vector spaces, namely to the dual of the space of the quasi-translations of the first and of the second 2-surface, respectively. Thus, even if we consider the disjoint union $\mathcal{S}_{1} \cup \mathcal{S}_{2}$ to surround a single physical system, then we can add the energy-momentum of the first to that of the second only if there is some physically/geometrically distinguished rule defining an isomorphism between the different vector spaces of the quasi-translations. Such an isomorphism would be provided for example by some naturally chosen globally defined flat background. However, as we discussed in Section 3.1.1, general relativity itself does not provide any background: The use of such a background contradicts the complete diffeomorphism invariance of the theory. Nevertheless, the quasi-local mass and the length of the quasi-local Pauli-Lubanski spin of different surfaces can be compared, because they are scalar quantities.

Similarly, any reasonable quasi-local angular momentum expression $J_{\mathcal{S}}^{\underline{a}}$ may be expected to satisfy the following:

Living Reviews in Relativity

http://www.livingreviews. org/lrr-2004-4 
$2.1 J_{\mathcal{S}}^{\underline{a}} \underline{b}$ must give zero for round spheres.

2.2 For 2-surfaces with zero quasi-local mass the Pauli-Lubanski spin should be proportional to the (null) energy-momentum 4 -vector $P_{\mathcal{S}}^{\underline{a}}$.

$2.3 J_{\mathcal{S}}^{\underline{a}}$ must give the correct weak field limit.

$2.4 J_{\mathcal{S}}^{\underline{a} \underline{b}}$ must reproduce the generally accepted spatial angular momentum at the spatial infinity, and in stationary spacetimes it should reduce to the 'standard' expression at the null infinity as well ('correct large sphere behaviour').

2.5 For small spheres the anti-self-dual part of $J_{\mathcal{S}}^{\underline{a}} \underline{\underline{b}}$, defined with respect to the centre of the small sphere (the 'vertex' in Section 4.2.2) is expected to give $\frac{4}{3} \pi r^{3} T_{c d} t^{c}\left(r \varepsilon^{D(A} t^{B) D^{\prime}}\right)$ in non-vacuum and $C r^{5} T_{c d e f} t^{c} t^{d} t^{e}\left(r \varepsilon^{F(A} t^{B)} F^{\prime}\right)$ in vacuum for some constant $C$ ('correct small sphere behaviour').

Since there is no generally accepted definition for the angular momentum at null infinity, we cannot expect anything definite there in non-stationary spacetimes. Similarly, there are inequivalent suggestions for the centre-of-mass at the spatial infinity (see Sections 3.2.2 and 3.2.4).

\subsubsection{Incompatibility of certain 'natural' expectations}

As Eardley noted in [131], probably no quasi-local energy definition exists which would satisfy all of his criteria. In fact, it is easy to see that this is the case. Namely, any quasi-local energy definition which reduces to the 'standard' expression for round spheres cannot be monotonic, as the closed Friedmann-Robertson-Walker or the $\Omega_{M, m}$ spacetimes show explicitly. The points where the monotonicity breaks down are the extremal (maximal or minimal) surfaces, which represent event horizon in the spacetime. Thus one may argue that since the event horizon hides a portion of spacetime, we cannot know the details of the physical state of the matter + gravity system behind the horizon. Hence, in particular, the monotonicity of the quasi-local mass may be expected to break down at the event horizon. However, although for stationary systems (or at the moment of time symmetry of a time-symmetric system) the event horizon corresponds to an apparent horizon (or to an extremal surface, respectively), for general non-stationary systems the concepts of the event and apparent horizons deviate. Thus the causal argument above does not seem possible to be formulated in the hypersurface $\Sigma$ of Section 4.3.2. Actually, the root of the non-monotonicity is the fact that the quasi-local energy is a 2-surface observable in the sense of Expectation 1 in Section 4.3.1 above. This does not mean, of course, that in certain restricted situations the monotonicity ('local monotonicity') could not be proven. This local monotonicity may be based, for example, on Lie dragging of the 2-surface along some special spacetime vector field.

On the other hand, in the literature sometimes the positivity and the monotonicity requirements are confused, and there is an 'argument' that the quasi-local gravitational energy cannot be positive definite, because the total energy of the closed universes must be zero. However, this argument is based on the implicit assumption that the quasi-local energy is associated with a compact three dimensional domain, which, together with the positive definiteness requirement would, in fact, imply the monotonicity and a positive total energy for the closed universe. If, on the other hand, the quasi-local energy-momentum is associated with 2-surfaces, then the energy may be positive definite and not monotonic. The standard round sphere energy expression (26) in the closed Friedmann-Robertson-Walker spacetime, or, more generally, the Dougan-Mason energymomentum (see Section 8.2.3) are such examples. 


\section{The Bartnik Mass and its Modifications}

\subsection{The Bartnik mass}

\subsubsection{The main idea}

One of the most natural ideas of quasi-localization of the familiar ADM mass is due to Bartnik [39, 38]. His idea is based on the positivity of the ADM energy, and, roughly, can be summarized as follows. Let $\Sigma$ be a compact, connected 3-manifold with connected boundary $\mathcal{S}$, and let $h_{a b}$ be a (negative definite) metric and $\chi_{a b}$ a symmetric tensor field on $\Sigma$ such that they, as an initial data set, satisfy the dominant energy condition: If $16 \pi G \mu:=R+\chi^{2}-\chi_{a b} \chi^{a b}$ and $8 \pi G j^{a}:=D_{b}\left(\chi^{a b}-\chi h^{a b}\right)$, then $\mu \geq\left(-j_{a} j^{a}\right)^{1 / 2}$. For the sake of simplicity we denote the triple $\left(\Sigma, h_{a b}, \chi_{a b}\right)$ by $\Sigma$. Then let us consider all the possible asymptotically flat initial data sets $\left(\hat{\Sigma}, \hat{h}_{a b}, \hat{\chi}_{a b}\right)$ with a single asymptotic end, denoted simply by $\hat{\Sigma}$, which satisfy the dominant energy condition, have finite ADM energy and are extensions of $\Sigma$ above through its boundary $\mathcal{S}$. The set of these extensions will be denoted by $\mathcal{E}(\Sigma)$. By the positive energy theorem $\hat{\Sigma}$ has non-negative ADM energy $E_{\mathrm{ADM}}(\hat{\Sigma})$, which is zero precisely when $\hat{\Sigma}$ is a data set for the flat spacetime. Then we can consider the infimum of the ADM energies, inf $\left\{E_{\mathrm{ADM}}(\hat{\Sigma}) \mid \hat{\Sigma} \in \mathcal{E}(\Sigma)\right\}$, where the infimum is taken on $\mathcal{E}(\Sigma)$. Obviously, by the non-negativity of the ADM energies this infimum exists and is non-negative, and it is tempting to define the quasi-local mass of $\Sigma$ by this infimum ${ }^{9}$. However, it is easy to see that, without further conditions on the extensions of $\left(\Sigma, h_{a b}, \chi_{a b}\right)$, this infimum is zero. In fact, $\Sigma$ can be extended to an asymptotically flat initial data set $\hat{\Sigma}$ with arbitrarily small ADM energy such that $\hat{\Sigma}$ contains a horizon (for example in the form of an apparent horizon) between the asymptotically flat end and $\Sigma$. In particular, in the ' $\Omega_{M, m}$-spacetime', discussed in Section 4.2.1 on the round spheres, the spherically symmetric domain bounded by the maximal surface (with arbitrarily large round-sphere mass $M / G$ ) has an asymptotically flat extension, the $\Omega_{M, m}$-spacetime itself, with arbitrarily small ADM mass $m / G$.

Obviously, the fact that the ADM energies of the extensions can be arbitrarily small is a consequence of the presence of a horizon hiding $\Sigma$ from the outside. This led Bartnik [39, 38] to formulate his suggestion for the quasi-local mass of $\Sigma$. He concentrated on the time-symmetric data sets (i.e. those for which the extrinsic curvature $\chi_{a b}$ is vanishing), when the horizon appears to be a minimal surface of topology $S^{2}$ in $\hat{\Sigma}$ (see for example [156]), and the dominant energy condition is just the requirement of the non-negativity of the scalar curvature: $R \geq 0$. Thus, if $\mathcal{E}_{0}(\Sigma)$ denotes the set of asymptotically flat Riemannian geometries $\hat{\Sigma}=\left(\hat{\Sigma}, \hat{h}_{a b}\right)$ with non-negative scalar curvature and finite ADM energy that contain no stable minimal surface, then Bartnik's mass is

$$
m_{\mathrm{B}}(\Sigma):=\inf \left\{E_{\mathrm{ADM}}(\hat{\Sigma}) \mid \hat{\Sigma} \in \mathcal{E}_{0}(\Sigma)\right\}
$$

The 'no-horizon' condition on $\hat{\Sigma}$ implies that topologically $\Sigma$ is a 3-ball. Furthermore, the definition of $\mathcal{E}_{0}(\Sigma)$ in its present form does not allow one to associate the Bartnik mass to those 3-geometries $\left(\Sigma, h_{a b}\right)$ that contain minimal surfaces inside $\Sigma$. Although formally the maximal 2-surfaces inside $\Sigma$ are not excluded, any asymptotically flat extension of such a $\Sigma$ would contain a minimal surface. In particular, the spherically symmetric 3-geometry with line element $d l^{2}=-d r^{2}-\sin ^{2} r\left(d \theta^{2}+\right.$ $\left.\sin ^{2} \theta d \phi^{2}\right)$ with $(\theta, \phi) \in S^{2}$ and $r \in\left[0, r_{0}\right], \pi / 2<r_{0}<\pi$, has a maximal 2-surface at $r=\pi / 2$, and any of its asymptotically flat extensions necessarily contains a minimal surface of area not greater than $4 \pi \sin ^{2} r_{0}$. Thus the Bartnik mass (according to the original definition given in [39, 38]) cannot be associated with every compact time-symmetric data set $\left(\Sigma, h_{a b}\right)$ even if $\Sigma$ is topologically trivial. Since for $0<r_{0}<\pi / 2$ this data set can be extended without any difficulty, this example shows that

\footnotetext{
${ }^{9}$ Since we take the infimum, we could equally take the ADM masses, which are the minimum values of the zero-th component of the energy-momentum four-vectors in the different Lorentz frames, instead of the energies.
}

Living Reviews in Relativity

http://www. livingreviews.org//rr-2004-4 
$m_{\mathrm{B}}$ is associated with the 3-dimensional data set $\Sigma$ and not only to the 2-dimensional boundary $\partial \Sigma$.

Of course, to rule out this limitation, one can modify the original definition by considering the set $\tilde{\mathcal{E}}_{0}(\mathcal{S})$ of asymptotically flat Riemannian geometries $\hat{\Sigma}=\left(\hat{\Sigma}, \hat{h}_{a b}\right)$ (with non-negative scalar curvature, finite $\mathrm{ADM}$ energy and with no stable minimal surface) which contain $\left(\mathcal{S}, q_{a b}\right)$ as an isometrically embedded Riemannian submanifold, and define $\tilde{m}_{\mathrm{B}}(\mathcal{S})$ by Equation $(36)$ with $\tilde{\mathcal{E}}_{0}(\mathcal{S})$ instead of $\mathcal{E}_{0}(\Sigma)$. Obviously, this $\tilde{m}_{\mathrm{B}}(\mathcal{S})$ could be associated with a larger class of 2-surfaces than the original $m_{\mathrm{B}}(\Sigma)$ to compact 3-manifolds, and $0 \leq \tilde{m}_{\mathrm{B}}(\partial \Sigma) \leq m_{\mathrm{B}}(\Sigma)$ holds.

In $[208,41]$ the set $\mathcal{E}_{0}(\Sigma)$ was allowed to include extensions $\Sigma$ of $\Sigma$ having boundaries as compact outermost horizons, whenever the corresponding ADM energies are still non-negative [159], and hence $m_{\mathrm{B}}(\Sigma)$ is still well-defined and non-negative. (For another definition for $\mathcal{E}_{0}(\Sigma)$ allowing horizons in the extensions but excluding them between $\Sigma$ and the asymptotic end, see [87] and Section 5.2 below.)

Bartnik suggested a definition for the quasi-local mass of a spacelike 2-surface $\mathcal{S}$ (together with its induced metric and the two extrinsic curvatures), too [39]. He considered those globally hyperbolic spacetimes $\hat{M}:=\left(\hat{M}, \hat{g}_{a b}\right)$ that satisfy the dominant energy condition, admit an asymptotically flat (metrically complete) Cauchy surface $\hat{\Sigma}$ with finite ADM energy, have no event horizon and in which $\mathcal{S}$ can be embedded with its first and second fundamental forms. Let $\mathcal{E}_{0}(\mathcal{S})$ denote the set of these spacetimes. Since the ADM energy $E_{\mathrm{ADM}}(\hat{M})$ is non-negative for any $\hat{M} \in \mathcal{E}_{0}(\mathcal{S})$ (and is zero precisely for flat $\hat{M}$ ), the infimum

$$
m_{\mathrm{B}}(\mathcal{S}):=\inf \left\{E_{\mathrm{ADM}}(\hat{M}) \mid \hat{M} \in \mathcal{E}_{0}(\mathcal{S})\right\}
$$

exists and is non-negative. Although it seems plausible that $m_{\mathrm{B}}(\partial \Sigma)$ is only the 'spacetime version' of $m_{\mathrm{B}}(\Sigma)$, without the precise form of the no-horizon conditions in $\mathcal{E}_{0}(\Sigma)$ and that in $\mathcal{E}_{0}(\mathcal{S})$ they cannot be compared even if the extrinsic curvature were allowed in the extensions $\hat{\Sigma}$ of $\Sigma$.

\subsubsection{The main properties of $m_{\mathrm{B}}(\Sigma)$}

The first immediate consequence of Equation (36) is the monotonicity of the Bartnik mass: If $\Sigma_{1} \subset \Sigma_{2}$, then $\mathcal{E}_{0}\left(\Sigma_{2}\right) \subset \mathcal{E}_{0}\left(\Sigma_{1}\right)$, and hence $m_{\mathrm{B}}\left(\Sigma_{1}\right) \leq m_{\mathrm{B}}\left(\Sigma_{2}\right)$. Obviously, by definition (36) one has $m_{\mathrm{B}}(\Sigma) \leq m_{\mathrm{ADM}}(\hat{\Sigma})$ for any $\hat{\Sigma} \in \mathcal{E}_{0}(\Sigma)$. Thus if $m$ is any quasi-local mass functional which is larger than $m_{\mathrm{B}}$ (i.e. which assigns a non-negative real to any $\Sigma$ such that $m(\Sigma) \geq m_{\mathrm{B}}(\Sigma)$ for any allowed $\Sigma)$, furthermore if $m(\Sigma) \leq m_{\mathrm{ADM}}(\hat{\Sigma})$ for any $\hat{\Sigma} \in \mathcal{E}_{0}(\Sigma)$, then by the definition of the infimum in Equation (36) one has $m_{\mathrm{B}}(\Sigma) \geq m(\Sigma)-\varepsilon \geq m_{\mathrm{B}}(\Sigma)-\varepsilon$ for any $\varepsilon>0$. Therefore, $m_{\mathrm{B}}$ is the largest mass functional satisfying $m_{\mathrm{B}}(\Sigma) \leq m_{\mathrm{ADM}}(\hat{\Sigma})$ for any $\hat{\Sigma} \in \mathcal{E}_{0}(\Sigma)$. Another interesting consequence of the definition of $m_{\mathrm{B}}$, due to W. Simon, is that if $\hat{\Sigma}$ is any asymptotically flat, time symmetric extension of $\Sigma$ with non-negative scalar curvature satisfying $m_{\mathrm{ADM}}(\hat{\Sigma})<m_{\mathrm{B}}(\Sigma)$, then there is a black hole in $\hat{\Sigma}$ in the form of a minimal surface between $\Sigma$ and the infinity of $\hat{\Sigma}$ (see for example [41]).

As we saw, the Bartnik mass is non-negative, and, obviously, if $\Sigma$ is flat (and hence is a data set for the flat spacetime), then $m_{\mathrm{B}}(\Sigma)=0$. The converse of this statement is also true [208]: If $m_{\mathrm{B}}(\Sigma)=0$, then $\Sigma$ is locally flat. The Bartnik mass tends to the ADM mass [208]: If $\left(\hat{\Sigma}, \hat{h}_{a b}\right)$ is an asymptotically flat Riemannian 3-geometry with non-negative scalar curvature and finite ADM mass $m_{\mathrm{ADM}}(\hat{\Sigma})$, and if $\left\{\Sigma_{n}\right\}, n \in \mathbb{N}$, is a sequence of solid balls of coordinate radius $n$ in $\hat{\Sigma}$, then $\lim _{n \rightarrow \infty} m_{\mathrm{B}}\left(\Sigma_{n}\right)=m_{\mathrm{ADM}}(\Sigma)$. The proof of these two results is based on the use of the Hawking energy (see Section 6.1), by means of which a positive lower bound for $m_{\mathrm{B}}(\Sigma)$ can be given near the non-flat points of $\Sigma$. In the proof of the second statement one must use the fact that the Hawking energy tends to the ADM energy, which, in the time-symmetric case, is just the ADM mass.

The proof that the Bartnik mass reduces to the 'standard expression' for round spheres is a nice application of the Riemannian Penrose inequality [208]: Let $\Sigma$ be a spherically symmetric 
László B. Szabados

Riemannian 3-geometry with spherically symmetric boundary $\mathcal{S}:=\partial \Sigma$. One can form its 'standard' round-sphere energy $E(\mathcal{S})$ (see Section 4.2.1), and take its spherically symmetric asymptotically flat vacuum extension $\hat{\Sigma}_{\text {SS }}$ (see $[39,41]$ ). By the Birkhoff theorem the exterior part of $\hat{\Sigma}_{\text {SS }}$ is a part of a $t=$ const. hypersurface of the vacuum Schwarzschild solution, and its ADM mass is just $E(\mathcal{S})$. Then any asymptotically flat extension $\hat{\Sigma}$ of $\Sigma$ can also be considered as (a part of) an asymptotically flat time-symmetric hypersurface with minimal surface, whose area is $16 \pi G^{2}$ $E_{\mathrm{ADM}}\left(\hat{\Sigma}_{\mathrm{SS}}\right)$. Thus by the Riemannian Penrose inequality [208] $E_{\mathrm{ADM}}(\hat{\Sigma}) \geq E_{\mathrm{ADM}}\left(\hat{\Sigma}_{\mathrm{SS}}\right)=E(\mathcal{S})$. Therefore, the Bartnik mass of $\Sigma$ is just the 'standard' round sphere expression $E(\mathcal{S})$.

\subsubsection{The computability of the Bartnik mass}

Since for any given $\Sigma$ the set $\mathcal{E}_{0}(\Sigma)$ of its extensions is a huge set, it is almost hopeless to parameterize it. Thus, by the very definition, it seems very difficult to compute the Bartnik mass for a given, specific $\left(\Sigma, h_{a b}\right)$. Without some computational method the potentially useful properties of $m_{\mathrm{B}}(\Sigma)$ would be lost from the working relativist's arsenal.

Such a computational method might be based on a conjecture of Bartnik [39, 41]: The infimum in definition (36) of the mass $m_{\mathrm{B}}(\Sigma)$ is realized by an extension $\left(\hat{\Sigma}, \hat{h}_{a b}\right)$ of $\left(\Sigma, h_{a b}\right)$ such that the exterior region, $\left(\hat{\Sigma}-\Sigma,\left.\hat{h}_{a b}\right|_{\hat{\Sigma}-\Sigma}\right)$, is static, the metric is Lipschitz-continuous across the 2 -surface $\partial \Sigma \subset \hat{\Sigma}$, and the mean curvatures of $\partial \Sigma$ of the two sides are equal. Therefore, to compute $m_{\mathrm{B}}$ for a given $\left(\Sigma, h_{a b}\right)$, one should find an asymptotically flat, static vacuum metric $\hat{h}_{a b}$ satisfying the matching conditions on $\partial \Sigma$, and the Bartnik mass is the ADM mass of $\hat{h}_{a b}$. As Corvino showed [119], if there is an allowed extension $\hat{\Sigma}$ of $\Sigma$ for which $m_{\mathrm{ADM}}(\hat{\Sigma})=m_{\mathrm{B}}(\Sigma)$, then the extension $\hat{\Sigma}-\bar{\Sigma}$ is static; furthermore, if $\Sigma_{1} \subset \Sigma_{2}, m_{\mathrm{B}}\left(\Sigma_{1}\right)=m_{\mathrm{B}}\left(\Sigma_{2}\right)$ and $\Sigma_{2}$ has an allowed extension $\hat{\Sigma}$ for which $m_{\mathrm{B}}\left(\Sigma_{2}\right)=m_{\mathrm{ADM}}(\hat{\Sigma})$, then $\Sigma_{2}-\bar{\Sigma}_{1}$ is static. Thus the proof of Bartnik's conjecture is equivalent to the proof of the existence of such an allowed extension. The existence of such an extension is proven in [267] for geometries $\left(\Sigma, h_{a b}\right)$ close enough to the Euclidean one and satisfying a certain reflection symmetry, but the general existence proof is still lacking. Bartnik's conjecture is that $\left(\Sigma, h_{a b}\right)$ determines this exterior metric uniquely [41]. He conjectures [39, 41] that a similar computation method can be found for the mass $m_{\mathrm{B}}(\mathcal{S})$, defined in Equation (37), too, where the exterior metric should be stationary. This second conjecture is also supported by partial results [120]: If $\left(\Sigma, h_{a b}, \chi_{a b}\right)$ is any compact vacuum data set, then it has an asymptotically flat vacuum extension which is a spacelike slice of a Kerr spacetime outside a large sphere near spatial infinity.

To estimate $m_{\mathrm{B}}(\Sigma)$ one can construct admissible extensions of $\left(\Sigma, h_{a b}\right)$ in the form of the metrics in quasi-spherical form [40]. If the boundary $\partial \Sigma$ is a metric sphere of radius $r$ with non-negative mean curvature $k$, then $m_{\mathrm{B}}(\Sigma)$ can be estimated from above in terms of $r$ and $k$.

\subsection{Bray's modifications}

Another, slightly modified definition for the quasi-local mass was suggested by Bray [87, 90]. Here we summarize his ideas.

Let $\Sigma=\left(\Sigma, h_{a b}, \chi_{a b}\right)$ be any asymptotically flat initial data set with finitely many asymptotic ends and finite ADM masses, and suppose that the dominant energy condition is satisfied on $\Sigma$. Let $\mathcal{S}$ be any fixed 2-surface in $\Sigma$ which encloses all the asymptotic ends except one, say the $i$-th (i.e. let $\mathcal{S}$ be homologous to a large sphere in the $i$-th asymptotic end). The outside region with respect to $\mathcal{S}$, denoted by $O(\mathcal{S})$, will be the subset of $\Sigma$ containing the $i$-th asymptotic end and bounded by $\mathcal{S}$, while the inside region, $I(\mathcal{S})$, is the (closure of) $\Sigma-O(\mathcal{S})$. Next Bray defines the 'extension' $\hat{\Sigma}_{e}$ of $\mathcal{S}$ by replacing $O(\mathcal{S})$ by a smooth asymptotically flat end of any data set satisfying the dominant energy condition. Similarly, the 'fill-in' $\hat{\Sigma}_{\mathrm{f}}$ of $\mathcal{S}$ is obtained from $\Sigma$ by replacing $I(\mathcal{S})$ by a smooth asymptotically flat end of any data set satisfying the dominant energy condition.

Living Reviews in Relativity

http: //www. livingreviews . org//rr-2004-4 
Finally, the surface $\mathcal{S}$ will be called outer-minimizing if for any closed 2-surface $\tilde{\mathcal{S}}$ enclosing $\mathcal{S}$ one has $\operatorname{Area}(\mathcal{S}) \leq \operatorname{Area}(\tilde{\mathcal{S}})$.

Let $\mathcal{S}$ be outer-minimizing, and let $\mathcal{E}(\mathcal{S})$ denote the set of extensions of $\mathcal{S}$ in which $\mathcal{S}$ is still outer-minimizing, and $\mathcal{F}(\mathcal{S})$ denote the set of fill-ins of $\mathcal{S}$. If $\hat{\Sigma}_{\mathrm{f}} \in \mathcal{F}(\mathcal{S})$ and $A_{\hat{\Sigma}_{\mathrm{f}}}$ denotes the infimum of the area of the 2-surfaces enclosing all the ends of $\hat{\Sigma}_{\mathrm{f}}$ except the outer one, then Bray defines the outer and inner mass, $m_{\text {out }}(\mathcal{S})$ and $m_{\text {in }}(\mathcal{S})$, respectively, by

$$
\begin{aligned}
m_{\text {out }}(\mathcal{S}) & :=\left\{m_{\mathrm{ADM}}\left(\hat{\Sigma}_{e}\right) \mid \hat{\Sigma}_{e} \in \mathcal{E}(\mathcal{S})\right\} \\
m_{\mathrm{in}}(\mathcal{S}) & :=\left\{A_{\hat{\Sigma}_{\mathrm{f}}} \mid \hat{\Sigma}_{\mathrm{f}} \in \mathcal{F}(\mathcal{S})\right\}
\end{aligned}
$$

$m_{\text {out }}(\mathcal{S})$ deviates slightly from Bartnik's mass $(36)$ even if the latter would be defined for non-timesymmetric data sets, because Bartnik's 'no-horizon condition' excludes apparent horizons from the extensions, while Bray's condition is that $\mathcal{S}$ be outer-minimizing.

A simple consequence of the definitions is the monotonicity of these masses: If $\mathcal{S}_{2}$ and $\mathcal{S}_{1}$ are outer-minimizing 2 -surfaces such that $\mathcal{S}_{2}$ encloses $\mathcal{S}_{1}$, then $m_{\text {in }}\left(\mathcal{S}_{2}\right) \geq m_{\text {in }}\left(\mathcal{S}_{1}\right)$ and $m_{\text {out }}\left(\mathcal{S}_{2}\right) \geq$ $m_{\text {out }}\left(\mathcal{S}_{1}\right)$. Furthermore, if the Penrose inequality holds (for example in a time-symmetric data set, for which the inequality has been proved), then for outer-minimizing surfaces $m_{\text {out }}(\mathcal{S}) \geq$ $m_{\text {in }}(\mathcal{S})[87,90]$. Furthermore, if $\Sigma_{i}$ is a sequence such that the boundaries $\partial \Sigma_{i}$ shrink to a minimal surface $\mathcal{S}$, then the sequence $m_{\text {out }}\left(\partial \Sigma_{i}\right)$ tends to the irreducible mass $\sqrt{\operatorname{Area}(\mathcal{S}) /\left(16 \pi G^{2}\right)}[41]$. Bray defines the quasi-local mass of a surface not simply to be a number, but the whole closed interval $\left[m_{\text {in }}(\mathcal{S}), m_{\text {out }}(\mathcal{S})\right]$. If $\mathcal{S}$ encloses the horizon in the Schwarzschild data set, then the inner and outer masses coincide, and Bray expects that the converse is also true: If $m_{\text {in }}(\mathcal{S})=m_{\text {out }}(\mathcal{S})$ then $\mathcal{S}$ can be embedded into the Schwarzschild spacetime with the given 2-surface data on $\mathcal{S}$ [90]. 


\section{The Hawking Energy and its Modifications}

\subsection{The Hawking energy}

\subsubsection{The definition}

Studying the perturbation of the dust-filled $k=-1$ Friedmann-Robertson-Walker spacetimes, Hawking found that

$$
\begin{aligned}
E_{\mathrm{H}}(\mathcal{S}): & =\sqrt{\frac{\operatorname{Area}(\mathcal{S})}{16 \pi G^{2}}}\left(1+\frac{1}{2 \pi} \oint_{\mathcal{S}} \rho \rho^{\prime} d \mathcal{S}\right)= \\
& =\sqrt{\frac{\operatorname{Area}(\mathcal{S})}{16 \pi G^{2}}} \frac{1}{4 \pi} \oint_{\mathcal{S}}\left(\sigma \sigma^{\prime}+\bar{\sigma} \bar{\sigma}^{\prime}-\psi_{2}-\bar{\psi}_{2^{\prime}}+2 \phi_{11}+2 \Lambda\right) d \mathcal{S}
\end{aligned}
$$

behaves as an appropriate notion of energy surrounded by the spacelike topological 2-sphere $\mathcal{S}$ [171]. Here we used the Gauss-Bonnet theorem and the GHP form of Equations $(22,23)$ for $F$ to express $\rho \rho^{\prime}$ by the curvature components and the shears. Thus the Hawking energy is genuinely quasi-local.

The Hawking energy has the following clear physical interpretation even in a general spacetime, and, in fact, $E_{\mathrm{H}}$ can be introduced in this way. Starting with the rough idea that the mass-energy surrounded by a spacelike 2 -sphere $\mathcal{S}$ should be the measure of bending of the ingoing and outgoing light rays orthogonal to $\mathcal{S}$, and recalling that under a boost gauge transformation $l^{a} \mapsto \alpha l^{a}$, $n^{a} \mapsto \alpha^{-1} n^{a}$ the convergences $\rho$ and $\rho^{\prime}$ transform as $\rho \mapsto \alpha \rho$ and $\rho^{\prime} \mapsto \alpha^{-1} \rho^{\prime}$, respectively, the energy must have the form $C+D \oint_{\mathcal{S}} \rho \rho^{\prime} d \mathcal{S}$, where the unspecified parameters $C$ and $D$ can be determined in some special situations. For metric 2-spheres of radius $r$ in the Minkowski spacetime, for which $\rho=-1 / r$ and $\rho^{\prime}=1 / 2 r$, we expect zero energy, thus $D=C / 2(\pi)$. For the event horizon of a Schwarzschild black hole with mass parameter $m$, for which $\rho=0=\rho^{\prime}$, we expect $m / G$, which can be expressed by the area of $\mathcal{S}$. Thus $C^{2}=\operatorname{Area}(\mathcal{S}) /\left(16 \pi G^{2}\right)$, and hence we arrive at Equation (38).

\subsubsection{The Hawking energy for spheres}

Obviously, for round spheres $E_{\mathrm{H}}$ reduces to the standard expression (26). This implies, in particular, that the Hawking energy is not monotonic in general. Since for a Killing horizon (e.g. for a stationary event horizon) $\rho=0$, the Hawking energy of its spacelike spherical cross sections $\mathcal{S}$ is $\sqrt{\operatorname{Area}(\mathcal{S}) /\left(16 \pi G^{2}\right)}$. In particular, for the event horizon of a Kerr-Newman black hole it is just the familiar irreducible mass $\sqrt{2 m^{2}-e^{2}+2 m \sqrt{m^{2}-e^{2}-a^{2}}} /(2 G)$.

For a small sphere of radius $r$ with centre $p \in M$ in non-vacuum spacetimes it is $\frac{4 \pi}{3} r^{3} T_{a b} t^{a} t^{b}$, while in vacuum it is $\frac{2}{45 G} r^{5} T_{a b c d} t^{a} t^{b} t^{c} t^{d}$, where $T_{a b}$ is the energy-momentum tensor and $T_{a b c d}$ is the Bel-Robinson tensor at $p$ [204]. The first result shows that in the lowest order the gravitational 'field' does not have a contribution to the Hawking energy, that is due exclusively to the matter fields. Thus in vacuum the leading order of $E_{\mathrm{H}}$ must be higher than $r^{3}$. Then even a simple dimensional analysis shows that the number of the derivatives of the metric in the coefficient of the $r^{k}$ order term in the power series expansion of $E_{\mathrm{H}}$ is $(k-1)$. However, there are no tensorial quantities built from the metric and its derivatives such that the total number of the derivatives involved would be three. Therefore, in vacuum, the leading term is necessarily of order $r^{5}$, and its coefficient must be a quadratic expression of the curvature tensor. It is remarkable that for small spheres $E_{\mathrm{H}}$ is positive definite both in non-vacuum (provided the matter fields satisfy, for example, the dominant energy condition) and vacuum. This shows, in particular, that $E_{\mathrm{H}}$ should be interpreted as energy rather than as mass: For small spheres in a $p p$-wave spacetime $E_{\mathrm{H}}$ is positive, while, as we saw this for the matter fields in Section 2.2.3, a mass expression could be expected to

Living Reviews in Relativity

http: //www . livingreviews . org/lrr-2004-4 
be zero. (We will see in Sections 8.2.3 and 13.5 that, for the Dougan-Mason energy-momentum, the vanishing of the mass characterizes the $p p$-wave metrics completely.)

Using the second expression in Equation (38) it is easy to see that at future null infinity $E_{\mathrm{H}}$ tends to the Bondi-Sachs energy. A detailed discussion of the asymptotic properties of $E_{\mathrm{H}}$ near null infinity, both for radiative and stationary spacetimes is given in [338, 340]. Similarly, calculating $E_{\mathrm{H}}$ for large spheres near spatial infinity in an asymptotically flat spacelike hypersurface, one can show that it tends to the ADM energy.

\subsubsection{Positivity and monotonicity properties}

In general the Hawking energy may be negative, even in the Minkowski spacetime. Geometrically this should be clear, since for an appropriately general (e.g. concave) 2-surface $\mathcal{S}$ the integral $\oint_{\mathcal{S}} \rho \rho^{\prime} d \mathcal{S}$ could be less than $-2 \pi$. Indeed, in flat spacetime $E_{\mathrm{H}}$ is proportional to $\oint_{\mathcal{S}}\left(\sigma \sigma^{\prime}+\bar{\sigma} \bar{\sigma}^{\prime}\right) d \mathcal{S}$ by the Gauss equation. For topologically spherical 2 -surfaces in the $t=$ const. spacelike hyperplane of Minkowski spacetime $\sigma \sigma^{\prime}$ is real and non-positive, and it is zero precisely for metric spheres, while for 2-surfaces in the $r=$ const. timelike cylinder $\sigma \sigma^{\prime}$ is real and non-negative, and it is zero precisely for metric spheres ${ }^{10}$. If, however, $\mathcal{S}$ is 'round enough' (not to be confused with the round spheres in Section 4.2.1), which is some form of a convexity condition, then $E_{\mathrm{H}}$ behaves nicely [111]: $\mathcal{S}$ will be called round enough if it is a submanifold of a spacelike hypersurface $\Sigma$, and if among the 2-dimensional surfaces in $\Sigma$ which enclose the same volume as $\mathcal{S}$ does, $\mathcal{S}$ has the smallest area. Then it is proven by Christodoulou and Yau [111] that if $\mathcal{S}$ is round enough in a maximal spacelike slice $\Sigma$ on which the energy density of the matter fields is non-negative (for example if the dominant energy condition is satisfied), then the Hawking energy is non-negative.

Although the Hawking energy is not monotonic in general, it has interesting monotonicity properties for special families of 2-surfaces. Hawking considered one-parameter families of spacelike 2 -surfaces foliating the outgoing and the ingoing null hypersurfaces, and calculated the change of $E_{\mathrm{H}}$ [171]. These calculations were refined by Eardley [131]. Starting with a weakly future convex 2 -surface $\mathcal{S}$ and using the boost gauge freedom, he introduced a special family $\mathcal{S}_{r}$ of spacelike 2 -surfaces in the outgoing null hypersurface $\mathcal{N}$, where $r$ will be the luminosity distance along the outgoing null generators. He showed that $E_{\mathrm{H}}\left(\mathcal{S}_{r}\right)$ is non-decreasing with $r$, provided the dominant energy condition holds on $\mathcal{N}$. Similarly, for weakly past convex $\mathcal{S}$ and the analogous family of surfaces in the ingoing null hypersurface $E_{\mathrm{H}}\left(\mathcal{S}_{r}\right)$ is non-increasing. Eardley also considered a special spacelike hypersurface, filled by a family of 2-surfaces, for which $E_{\mathrm{H}}\left(\mathcal{S}_{r}\right)$ is non-decreasing. By relaxing the normalization condition $l_{a} n^{a}=1$ for the two null normals to $l_{a} n^{a}=\exp (f)$ for some $f: \mathcal{S} \rightarrow \mathbb{R}$, Hayward obtained a flexible enough formalism to introduce a double-null foliation (see Section 11.2 below) of a whole neighbourhood of a mean convex 2 -surface by special mean convex 2-surfaces [182]. (For the more general GHP formalism in which $l_{a} n^{a}$ is not fixed, see [312].) Assuming that the dominant energy condition holds, he showed that the Hawking energy of these 2 -surfaces is non-decreasing in the outgoing, and non-increasing in the ingoing direction.

In contrast to the special foliations of the null hypersurfaces above, Frauendiener defined a special spacelike vector field, the inverse mean curvature vector in the spacetime [145]. If $\mathcal{S}$ is a weakly future and past convex 2 -surface, then $q^{a}:=2 Q^{a} /\left(Q_{b} Q^{b}\right)=-[1 /(2 \rho)] l^{a}-\left[1 /\left(2 \rho^{\prime}\right)\right] n^{a}$ is an outward directed spacelike normal to $\mathcal{S}$. Here $Q_{b}$ is the trace of the extrinsic curvature tensor: $Q_{b}:=Q_{a b}^{a}$ (see Section 4.1.2). Starting with a single weakly future and past convex 2-surface, Frauendiener gives an argument for the construction of a one-parameter family $\mathcal{S}_{t}$ of 2-surfaces being Lie-dragged along its own inverse mean curvature vector $q^{a}$. Hence this family of surfaces would be analogous to the solution of the geodesic equation, where the initial point and direction in that point specify the whole solution, at least locally. Assuming that such a family of surfaces (and hence the vector field $q^{a}$ on the 3 -submanifold swept by $\mathcal{S}_{t}$ ) exists, Frauendiener showed that

\footnotetext{
${ }^{10}$ I thank Paul Tod for pointing this out to me.
} 
the Hawking energy is non-decreasing along the vector field $q^{a}$ if the dominant energy condition is satisfied. However, no investigation has been made to prove the existence of such a family of surfaces. Motivated by this result, Malec, Mars, and Simon [261] considered spacelike hypersurfaces with an inverse mean curvature flow of Geroch thereon (see Section 6.2.2). They showed that if the dominant energy condition and certain additional (essentially technical) assumptions hold, then the Hawking energy is monotonic. These two results are the natural adaptations for the Hawking energy of the corresponding results known for some time for the Geroch energy, aiming to prove the Penrose inequality. We return to this latter issue in Section 13.2 only for a very brief summary.

\subsubsection{Two generalizations}

Hawking defined not only energy, but spatial momentum as well, completely analogously to how the spatial components of the Bondi-Sachs energy-momentum are related to the Bondi energy:

$$
P_{\overline{\mathrm{H}}}^{\underline{a}}(\mathcal{S})=\sqrt{\frac{\operatorname{Area}(\mathcal{S})}{16 \pi G^{2}}} \frac{1}{4 \pi} \oint_{\mathcal{S}}\left(\sigma \sigma^{\prime}+\bar{\sigma} \bar{\sigma}^{\prime}-\psi_{2}-\bar{\psi}_{2^{\prime}}+2 \phi_{11}+2 \Lambda\right) W^{\underline{a}} d \mathcal{S}
$$

where $W^{\underline{a}}, \underline{a}=0, \ldots, 3$, are essentially the first four spherical harmonics:

$$
W^{0}=1, \quad W^{1}=\frac{\zeta+\bar{\zeta}}{1+\zeta \bar{\zeta}}, \quad W^{2}=\frac{1}{\mathrm{i}} \frac{\zeta-\bar{\zeta}}{1+\zeta \bar{\zeta}}, \quad W^{3}=\frac{1-\zeta \bar{\zeta}}{1+\zeta \bar{\zeta}}
$$

Here $\zeta$ and $\bar{\zeta}$ are the standard complex stereographic coordinates on $\mathcal{S} \approx S^{2}$.

Hawking considered the extension of the definition of $E_{\mathrm{H}}(\mathcal{S})$ to higher genus 2-surfaces also by the second expression in Equation (38). Then in the expression analogous to the first one in Equation (38) the genus of $\mathcal{S}$ appears.

\subsection{The Geroch energy}

\subsubsection{The definition}

Suppose that the 2-surface $\mathcal{S}$ for which $E_{\mathrm{H}}$ is defined is embedded in the spacelike hypersurface $\Sigma$. Let $\chi_{a b}$ be the extrinsic curvature of $\Sigma$ in $M$ and $k_{a b}$ the extrinsic curvature of $\mathcal{S}$ in $\Sigma$. (In Section 4.1 .2 we denoted the latter by $\nu_{a b}$. $)$ Then $8 \rho \rho^{\prime}=\left(\chi_{a b} q^{a b}\right)^{2}-\left(k_{a b} q^{a b}\right)^{2}$, by means of which

$$
\begin{aligned}
E_{\mathrm{H}}(\mathcal{S}) & =\sqrt{\frac{\operatorname{Area}(\mathcal{S})}{16 \pi G^{2}}}\left(1-\frac{1}{16 \pi} \oint_{\mathcal{S}}\left(k_{a b} q^{a b}\right)^{2} d \mathcal{S}+\frac{1}{16 \pi} \oint_{\mathcal{S}}\left(\chi_{a b} q^{a b}\right)^{2} d \mathcal{S}\right) \geq \\
& \geq \sqrt{\frac{\operatorname{Area}(\mathcal{S})}{16 \pi G^{2}}}\left(1-\frac{1}{16 \pi} \oint_{\mathcal{S}}\left(k_{a b} q^{a b}\right)^{2} d \mathcal{S}\right)= \\
& =\frac{1}{16 \pi} \sqrt{\frac{\operatorname{Area}(\mathcal{S})}{16 \pi G^{2}}} \oint_{\mathcal{S}}\left(2^{\mathcal{S}} R-\left(k_{a b} q^{a b}\right)^{2}\right) d \mathcal{S}=: E_{\mathrm{G}}(\mathcal{S})
\end{aligned}
$$

In the last step we used the Gauss-Bonnet theorem for $\mathcal{S} \approx S^{2} . E_{\mathrm{G}}(\mathcal{S})$ is known as the Geroch energy [150]. Thus it is not greater than the Hawking energy, and, in contrast to $E_{\mathrm{H}}$, it depends not only on the 2 -surface $\mathcal{S}$, but the hypersurface $\Sigma$ as well.

The calculation of the small sphere limit of the Geroch energy was saved by observing [204] that, by Equation (41), the difference of the Hawking and the Geroch energies is proportional to $\sqrt{\operatorname{Area}(\mathcal{S})} \times \oint_{\mathcal{S}}\left(\chi_{a b} q^{a b}\right)^{2} d \mathcal{S}$. Since, however, $\chi_{a b} q^{a b}-$ for the family of small spheres $\mathcal{S}_{r}-$ does not tend to zero in the $r \rightarrow 0$ limit, in general this difference is $\mathcal{O}\left(r^{3}\right)$. It is zero if $\Sigma$ is spanned by spacelike geodesics orthogonal to $t^{a}$ at $p$. Thus, for general $\Sigma$, the Geroch energy does not

Living Reviews in Relativity

http: //www . livingreviews . org/lrr-2004-4 
give the expected $\frac{4 \pi}{3} r^{3} T_{a b} t^{a} t^{b}$ result. Similarly, in vacuum the Geroch energy deviates from the Bel-Robinson energy in $r^{5}$ order even if $\Sigma$ is geodesic at $p$.

Since $E_{\mathrm{H}}(\mathcal{S}) \geq E_{\mathrm{G}}(\mathcal{S})$ and since the Hawking energy tends to the ADM energy, the large sphere limit of $E_{\mathrm{G}}(\mathcal{S})$ in an asymptotically flat $\Sigma$ cannot be greater than the ADM energy. In fact, it is also precisely the ADM energy [150].

\subsubsection{Monotonicity properties}

The Geroch energy has interesting positivity and monotonicity properties along a special flow in $\Sigma[150,219]$. This flow is the so-called inverse mean curvature flow defined as follows. Let $t: \Sigma \rightarrow \mathbb{R}$ be a smooth function such that

1. its level surfaces, $\mathcal{S}_{t}:=\{q \in \Sigma \mid t(q)=t\}$, are homeomorphic to $S^{2}$,

2. there is a point $p \in \Sigma$ such that the surfaces $\mathcal{S}_{t}$ are shrinking to $p$ in the limit $t \rightarrow-\infty$, and

3. they form a foliation of $\Sigma-\{p\}$.

Let $n$ be the lapse function of this foliation, i.e. if $v^{a}$ is the outward directed unit normal to $\mathcal{S}_{t}$ in $\Sigma$, then $n v^{a} D_{a} t=1$. Denoting the integral on the right hand side in Equation (41) by $W_{t}$, we can calculate its derivative with respect to $t$. In general this derivative does not seem to have any remarkable property. If, however, the foliation is chosen in a special way, namely if the lapse is just the inverse mean curvature of the foliation, $n=1 / k$ where $k:=k_{a b} q^{a b}$, furthermore $\Sigma$ is maximal (i.e. $\chi=0$ ) and the energy density of the matter is non-negative, then, as shown by Geroch [150], $W_{t} \geq 0$ holds. Jang and Wald [219] modified the foliation slightly such that $t \in[0, \infty)$, and the surface $\mathcal{S}_{0}$ was assumed to be future marginally trapped (i.e. $\rho=0$ and $\rho^{\prime} \geq 0$ ). Then they showed that, under the conditions above, $\sqrt{\operatorname{Area}\left(\mathcal{S}_{0}\right)} W_{0} \leq \sqrt{\operatorname{Area}\left(\mathcal{S}_{t}\right)} W_{t}$. Since $E_{\mathrm{G}}\left(\mathcal{S}_{t}\right)$ tends to the ADM energy as $t \rightarrow \infty$, these considerations were intended to argue that the ADM energy should be non-negative (at least for maximal $\Sigma$ ) and not less than $\sqrt{\operatorname{Area}\left(\mathcal{S}_{0}\right) /\left(16 \pi G^{2}\right)}$ (at least for time-symmetric $\Sigma$ ), respectively. Later Jang [217] showed that if a certain quasi-linear elliptic differential equation for a function $w$ on a hypersurface $\Sigma$ admits a solution (with given asymptotic behaviour), then $w$ defines a mapping between the data set $\left(\Sigma, h_{a b}, \chi_{a b}\right)$ on $\Sigma$ and a maximal data set $\left(\Sigma, \bar{h}_{a b}, \bar{\chi}_{a b}\right)$ (i.e. for which $\left.\bar{\chi}_{a b} \bar{h}^{a b}=0\right)$ such that the corresponding ADM energies coincide. Then Jang shows that a slightly modified version of the Geroch energy is monotonic (and tends to the ADM energy) with respect to a new, modified version of the inverse mean curvature foliation of $\left(\Sigma, \bar{h}_{a b}\right)$.

The existence and the properties of the original inverse mean curvature foliation of $\left(\Sigma, h_{a b}\right)$ above were proven and clarified by Huisken and Ilmanen [207, 208], giving the first complete proof of the Riemannian Penrose inequality, and, as proved by Schoen and Yau [328], Jang's quasi-linear elliptic equation admits a global solution.

\subsection{The Hayward energy}

We saw that $E_{\mathrm{H}}$ can be non-zero even in the Minkowski spacetime. This may motivate considering the following expression

$$
\begin{aligned}
I(\mathcal{S}) & :=\sqrt{\frac{\operatorname{Area}(\mathcal{S})}{16 \pi G^{2}}}\left(1+\frac{1}{4 \pi} \oint_{\mathcal{S}}\left(2 \rho \rho^{\prime}-\sigma \sigma^{\prime}-\bar{\sigma} \bar{\sigma}^{\prime}\right) d \mathcal{S}\right)= \\
& =\sqrt{\frac{\operatorname{Area}(\mathcal{S})}{16 \pi G^{2}}} \frac{1}{4 \pi} \oint_{\mathcal{S}}\left(-\psi_{2}-\bar{\psi}_{2^{\prime}}+2 \phi_{11}+2 \Lambda\right) d \mathcal{S} .
\end{aligned}
$$

(Thus the integrand is $\frac{1}{4}(F+\bar{F})$, where $F$ is given by Equation (23).) By the Gauss equation this is zero in flat spacetime, furthermore, it is not difficult to see that its limit at the spatial infinity 
is still the $\mathrm{ADM}$ energy. However, using the second expression of $I(\mathcal{S})$, one can see that its limit at the future null infinity is the Newman-Unti rather than the Bondi-Sachs energy.

In the literature there is another modification of the Hawking energy, due to Hayward [183]. His suggestion is essentially $I(\mathcal{S})$ with the only difference that the integrands above contain an additional term, namely the square of the anholonomicity $-\omega_{a} \omega^{a}$ (see Sections 4.1.8 and 11.2.1). However, we saw that $\omega_{a}$ is a boost gauge dependent quantity, thus the physical significance of this suggestion is questionable unless a natural boost gauge choice, e.g. in the form of a preferred foliation, is made. (Such a boost gauge might be that given by the main extrinsic curvature vector $Q_{a}$ and $\tilde{Q}_{a}$ discussed in Section 4.1.2.) Although the expression for the Hayward energy in terms of the GHP spin coefficients given in $[63,65]$ seems to be gauge invariant, this is due only to an implicit gauge choice. The correct, general GHP form of the extra term is $-\omega_{a} \omega^{a}=2\left(\beta-\bar{\beta}^{\prime}\right)\left(\bar{\beta}-\beta^{\prime}\right)$. If, however, the GHP spinor dyad is fixed as in the large sphere or in the small sphere calculations, then $\beta-\bar{\beta}^{\prime}=\tau=-\bar{\tau}^{\prime}$, and hence the extra term is, in fact, $2 \tau \bar{\tau}$.

Taking into account that $\tau=\mathcal{O}\left(r^{-2}\right)$ near the future null infinity (see for example [338]), it is immediate from the remark on the asymptotic behaviour of $I(\mathcal{S})$ above that the Hayward energy tends to the Newman-Unti instead of the Bondi-Sachs energy at the future null infinity. The Hayward energy has been calculated for small spheres both in non-vacuum and vacuum [63]. In non-vacuum it gives the expected value $\frac{4 \pi}{3} r^{3} T_{a b} t^{a} t^{b}$. However, in vacuum it is $-\frac{8}{45 G} r^{5} T_{a b c d} t^{a} t^{b} t^{c} t^{d}$, which is negative.

Living Reviews in Relativity

http: //www . livingreviews . org//rr-2004-4 


\section{Penrose's Quasi-Local Energy-Momentum and Angular Momentum}

The construction of Penrose is based on twistor-theoretical ideas, and motivated by the linearized gravity integrals for energy-momentum and angular momentum. Since, however, twistor-theoretical ideas and basic notions are still considered to be some 'special knowledge', the review of the basic idea behind the Penrose construction is slightly more detailed than that of the others. The basic references of the field are the volumes [312, 313] by Penrose and Rindler on 'Spinors and Spacetime', especially volume 2, the very well readable book by Hugget and Tod [206] and the comprehensive review article [377] by Tod.

\subsection{Motivations}

\subsubsection{How do the twistors emerge?}

In the Newtonian theory of gravity the mass contained in some finite 3-volume $\Sigma$ can be expressed as the flux integral of the gravitational field strength on the boundary $\mathcal{S}:=\partial \Sigma$ :

$$
m_{\Sigma}=\frac{1}{4 \pi G} \oint_{\mathcal{S}} v^{a}\left(D_{a} \phi\right) d \mathcal{S}
$$

where $\phi$ is the gravitational potential and $v^{a}$ is the outward directed unit normal to $\mathcal{S}$. If $\mathcal{S}$ is deformed in $\Sigma$ through a source-free region, then the mass does not change. Thus the mass $m_{\Sigma}$ is analogous to charge in electrostatics.

In the weak field (linear) approximation of general relativity on Minkowski spacetime the source of the gravitational field, i.e. the linearized energy-momentum tensor, is still analogous to charge. In fact, the total energy-momentum and angular momentum of the source can be expressed as appropriate 2-surface integrals of the curvature at infinity [349]. Thus it is natural to expect that the energy-momentum and angular momentum of the source in a finite 3 -volume $\Sigma$, given by Equation (5), can also be expressed as the charge integral of the curvature on the 2-surface $\mathcal{S}$. However, the curvature tensor can be integrated on $\mathcal{S}$ only if at least one pair of its indices is annihilated by some tensor via contraction, i.e. according to Equation (15) if some $\omega^{a b}=\omega^{[a b]}$ is chosen and $\mu^{a b}=\varepsilon^{a b}$. To simplify the subsequent analysis $\omega^{a b}$ will be chosen to be anti-self-dual: $\omega^{a b}=\varepsilon^{A^{\prime} B^{\prime}} \omega^{A B}$ with $\omega^{A B}=\omega^{(A B)}{ }^{11}$. Thus our claim is to find an appropriate spinor field $\omega^{A B}$ on $\mathcal{S}$ such that

$$
Q_{\mathcal{S}}[\mathbf{K}]:=\int_{\Sigma} K_{a} T^{a b} \frac{1}{3 !} \varepsilon_{b c d e}=\frac{1}{8 \pi G} \oint_{\mathcal{S}} \omega^{A B} R_{A B c d}=: A_{\mathcal{S}}[\omega] .
$$

Since the dual of the exterior derivative of the integrand on the right, and, by Einstein's equations, the dual of the $8 \pi G$ times the integrand on the left, respectively, is

$$
\begin{aligned}
\varepsilon^{e c d f} \nabla_{e}\left(\omega^{A B} R_{A B c d}\right) & =-2 \mathrm{i} \psi_{A B C}^{F} \nabla^{F^{\prime}(A} \omega^{B C)}+2 \phi_{A B E^{\prime}}{ }^{\prime} \mathrm{i} \nabla^{E^{\prime} F} \omega^{A B}+4 \Lambda \mathrm{i} \nabla_{A}^{F^{\prime}} \omega^{F A}, \\
-8 \pi G K_{a} T^{a f} & =2 \phi^{F A F^{\prime} A^{\prime}} K_{A A^{\prime}}+6 \Lambda K^{F F^{\prime}},
\end{aligned}
$$

Equations (44) and (45) are equal if $\omega^{A B}$ satisfies

$$
\nabla^{A^{\prime} A} \omega^{B C}=-\mathrm{i} \varepsilon^{A(B} K^{C) A^{\prime}} .
$$

This equation in its symmetrized form, $\nabla^{A^{\prime}\left(A \omega^{B C}\right)}=0$, is the valence 2 twistor equation, a specific example for the general twistor equation $\nabla^{A^{\prime}(A} \omega^{B C \ldots E)}=0$ for $\omega^{B C \ldots E}=\omega^{(B C \ldots E)}$. Thus, as could

\footnotetext{
${ }^{11}$ The analogous calculations using tensor methods and the real $\omega^{a b}$ instead of spinors and the anti-self-dual (or, shortly, a.s.d.) part of $\omega^{a b}$ would be technically more complicated [307, 308, 313, 164].
} 
be expected, $\omega^{A B}$ depends on the Killing vector $K^{a}$, and, in fact, $K^{a}$ can be recovered from $\omega^{A B}$ as $K^{A^{\prime} A}=\frac{2}{3} \mathrm{i} \nabla_{B}^{A^{\prime}} \omega^{A B}$. Thus $\omega^{A B}$ plays the role of a potential for the Killing vector $K^{A^{\prime} A}$. However, as a consequence of Equation (46), $K_{a}$ is a self-dual Killing 1-form in the sense that its derivative is a self-dual (or s.d.) 2-form: In fact, the general solution of equation (46) and the corresponding Killing vector are

$$
\begin{aligned}
\omega^{A B} & =-\mathrm{i} x^{A A^{\prime}} x^{B B^{\prime}} \bar{M}_{A^{\prime} B^{\prime}}+\mathrm{i} x_{A^{\prime}}^{(A} T^{B) A^{\prime}}+\Omega^{A B}, \\
K^{A A^{\prime}} & =T^{A A^{\prime}}+2 x^{A B^{\prime}} \bar{M}_{B^{\prime}}^{A^{\prime}},
\end{aligned}
$$

where $\bar{M}_{A^{\prime} B^{\prime}}, T^{A A^{\prime}}$, and $\Omega^{A B}$ are constant spinors, using the notation $x^{A A^{\prime}}:=x^{\underline{a}} \sigma_{\underline{a}}^{\underline{A}} \underline{A}^{\prime} \mathcal{E}_{\underline{A}}^{A} \overline{\mathcal{E}}_{\underline{A}}^{A^{\prime}}$, where $\left\{\mathcal{E}_{A}^{A}\right\}$ is a constant spin frame (the 'Cartesian spin frame') and $\sigma_{\underline{a}}^{\underline{A}} \underline{A}^{\prime}$ are the standard $S L(2, \mathbb{C})$ Pauli matrices (divided by $\sqrt{2}$ ). These yield that $K_{a}$ is, in fact, self-dual, $\nabla_{A A^{\prime}} K_{B B^{\prime}}=$ $\varepsilon_{A B} \bar{M}_{A^{\prime} B^{\prime}}$, and $T^{A A^{\prime}}$ is a translation and $\bar{M}_{A^{\prime} B^{\prime}}$ generates self-dual rotations. Then $Q_{\mathcal{S}}[\mathbf{K}]=$ $T_{A A^{\prime}} P^{A A^{\prime}}+2 \bar{M}_{A^{\prime} B^{\prime}} \bar{J}^{A^{\prime} B^{\prime}}$, implying that the charges corresponding to $\Omega^{A B}$ are vanishing; the four components of the quasi-local energy-momentum correspond to the real $T^{A A^{\prime}} \mathrm{s}$, and the spatial angular momentum and centre-of-mass are combined into the three complex components of the self-dual angular momentum $\bar{J}^{A^{\prime} B^{\prime}}$, generated by $\bar{M}_{A^{\prime} B^{\prime}}$.

\subsubsection{Twistor space and the kinematical twistor}

Recall that the space of the contravariant valence 1 twistors of Minkowski spacetime is the set of the pairs $Z^{\alpha}:=\left(\lambda^{A}, \pi_{A^{\prime}}\right)$ of spinor fields, which solve the so-called valence 1 twistor equation $\nabla^{A^{\prime} A} \lambda^{B}=-\mathrm{i} \varepsilon^{A B} \pi^{A^{\prime}}$. If $Z^{\alpha}$ is a solution of this equation, then $\hat{Z}^{\alpha}:=\left(\lambda^{A}, \pi_{A^{\prime}}+\mathrm{i} \Upsilon_{A^{\prime} A^{A}} \lambda^{A}\right)$ is a solution of the corresponding equation in the conformally rescaled spacetime, where $\Upsilon_{a}:=$ $\Omega^{-1} \nabla_{a} \Omega$ and $\Omega$ is the conformal factor. In general the twistor equation has only the trivial solution, but in the (conformal) Minkowski spacetime it has a four complex parameter family of solutions. Its general solution in the Minkowski spacetime is $\lambda^{A}=\Lambda^{A}-\mathrm{i} x^{A A^{\prime}} \pi_{A^{\prime}}$, where $\Lambda^{A}$ and $\pi_{A^{\prime}}$ are constant spinors. Thus the space $\mathbf{T}^{\alpha}$ of valence 1 twistors, the so-called twistor-space, is $4-$ complex-dimensional, and hence has the structure $\mathbf{T}^{\alpha}=\mathbf{S}^{A} \oplus \overline{\mathbf{S}}_{A^{\prime}} . \mathbf{T}^{\alpha}$ admits a natural Hermitian scalar product: If $W^{\beta}=\left(\omega^{B}, \sigma_{B^{\prime}}\right)$ is another twistor, then $H_{\alpha \beta^{\prime}} Z^{\alpha} \bar{W}^{\beta^{\prime}}:=\lambda^{A} \bar{\sigma}_{A}+\pi_{A^{\prime}} \bar{\omega}^{A^{\prime}}$. Its signature is $(+,+,-,-)$, it is conformally invariant, $H_{\alpha \beta^{\prime}} \hat{Z}^{\alpha} \overline{\hat{W}}^{\beta^{\prime}}=H_{\alpha \beta^{\prime}} Z^{\alpha} \bar{W}^{\beta^{\prime}}$, and it is constant on Minkowski spacetime. The metric $H_{\alpha \beta^{\prime}}$ defines a natural isomorphism between the complex conjugate twistor space, $\overline{\mathbf{T}}^{\alpha^{\prime}}$, and the dual twistor space, $\mathbf{T}_{\beta}:=\mathbf{S}_{B} \oplus \overline{\mathbf{S}}^{B^{\prime}}$, by $\left(\bar{\lambda}^{A^{\prime}}, \bar{\pi}_{A}\right) \mapsto\left(\bar{\pi}_{A}, \bar{\lambda}^{A^{\prime}}\right)$. This makes it possible to use only twistors with unprimed indices. In particular, the complex conjugate $\bar{A}_{\alpha^{\prime} \beta^{\prime}}$ of the covariant valence 2 twistor $A_{\alpha \beta}$ can be represented by the so-called conjugate twistor $\bar{A}^{\alpha \beta}:=\bar{A}_{\alpha^{\prime} \beta^{\prime}} H^{\alpha^{\prime} \alpha} H^{\beta^{\prime} \beta}$. We should mention two special, higher valence twistors. The first is the so-called infinity twistor. This and its conjugate are given explicitly by

$$
I^{\alpha \beta}:=\left(\begin{array}{cc}
\varepsilon^{A B} & 0 \\
0 & 0
\end{array}\right), \quad I_{\alpha \beta}:=\bar{I}^{\alpha^{\prime} \beta^{\prime}} H_{\alpha^{\prime} \alpha} H_{\beta^{\prime} \beta}=\left(\begin{array}{cc}
0 & 0 \\
0 & \varepsilon^{A^{\prime} B^{\prime}}
\end{array}\right) .
$$

The other is the completely anti-symmetric twistor $\varepsilon_{\alpha \beta \gamma \delta}$, whose component $\varepsilon_{0123}$ in an $H_{\alpha \beta^{\prime-}}$ orthonormal basis is required to be one. The only non-vanishing spinor parts of $\varepsilon_{\alpha \beta \gamma \delta}$ are those with two primed and two unprimed spinor indices: $\varepsilon^{A^{\prime} B^{\prime}}{ }_{C D}=\varepsilon^{A^{\prime} B^{\prime}} \varepsilon_{C D}, \varepsilon^{A^{\prime}}{ }_{B} C^{C^{\prime}}{ }_{D^{\prime}}=-\varepsilon^{A^{\prime} C^{\prime}} \varepsilon_{B D}$, $\varepsilon_{A B} C^{C^{\prime} D^{\prime}}=\varepsilon_{A B} \varepsilon_{C^{\prime} D^{\prime}}, \ldots$ Thus for any four twistors $Z_{i}^{\alpha}=\left(\lambda_{i}^{A}, \pi_{A^{\prime}}^{i}\right), i=1, \ldots, 4$, the determinant of the $4 \times 4$ matrix whose $i$-th column is $\left(\lambda_{i}^{\mathbf{0}}, \lambda_{i}^{\mathbf{1}}, \pi_{\mathbf{0}^{\prime}}^{i}, \pi_{\mathbf{1}^{\prime}}^{i}\right)$, where the $\lambda_{i}^{\mathbf{0}}, \ldots, \pi_{\mathbf{1}^{\prime}}^{i}$ are the components

Living Reviews in Relativity

http: //www . livingreviews . org/lrr-2004-4 
of the spinors $\lambda_{i}^{A}$ and $\pi_{A^{\prime}}^{i}$ in some spin frame, is

$$
\nu:=\operatorname{det}\left(\begin{array}{cccc}
\lambda_{1}^{0} & \lambda_{2}^{0} & \lambda_{3}^{0} & \lambda_{4}^{\mathbf{0}} \\
\lambda_{1}^{\mathbf{1}} & \lambda_{2}^{\mathbf{1}} & \lambda_{3}^{\mathbf{1}} & \lambda_{4}^{\mathbf{1}} \\
\pi_{\mathbf{0}^{\prime}}^{1} & \pi_{\mathbf{0}^{\prime}}^{2} & \pi_{\mathbf{0}^{\prime}}^{3} & \pi_{\mathbf{0}^{\prime}}^{4} \\
\pi_{\mathbf{1}^{\prime}}^{1} & \pi_{\mathbf{1}^{\prime}}^{2} & \pi_{\mathbf{1}^{\prime}}^{3} & \pi_{\mathbf{1}^{\prime}}^{4}
\end{array}\right)=\frac{1}{4} \epsilon^{i j}{ }_{k l} \lambda_{i}^{A} \lambda_{j}^{B} \pi_{A^{\prime}}^{k} \pi_{B^{\prime}}^{l} \varepsilon_{A B} \varepsilon^{A^{\prime} B^{\prime}}=\frac{1}{4} \varepsilon_{\alpha \beta \gamma \delta} Z_{1}^{\alpha} Z_{2}^{\beta} Z_{3}^{\gamma} Z_{4}^{\delta},
$$

where $\epsilon^{i j}{ }_{k l}$ is the totally antisymmetric Levi-Civita symbol. Then $I^{\alpha \beta}$ and $I_{\alpha \beta}$ are dual to each other in the sense that $I^{\alpha \beta}=\frac{1}{2} \varepsilon^{\alpha \beta \gamma \delta} I_{\gamma \delta}$, and by the simplicity of $I^{\alpha \beta}$ one has $\varepsilon_{\alpha \beta \gamma \delta} I^{\alpha \beta} I^{\gamma \delta}=0$.

The solution $\omega^{A B}$ of the valence 2 twistor equation, given by Equation (47), can always be written as a linear combination of the symmetrized product $\lambda^{(A} \omega^{B)}$ of the solutions $\lambda^{A}$ and $\omega^{A}$ of the valence 1 twistor equation. $\omega^{A B}$ defines uniquely a symmetric twistor $\omega^{\alpha \beta}$ (see for example [313]). Its spinor parts are

$$
\omega^{\alpha \beta}=\left(\begin{array}{cc}
\omega^{A B} & -\frac{1}{2} K^{A}{ }_{B^{\prime}} \\
-\frac{1}{2} K_{A^{\prime}}{ }^{B} & -\mathrm{i} \bar{M}_{A^{\prime} B^{\prime}}
\end{array}\right) .
$$

However, Equation (43) can be interpreted as a $\mathbb{C}$-linear mapping of $\omega^{\alpha \beta}$ into $\mathbb{C}$, i.e. Equation (43) defines a dual twistor, the (symmetric) kinematical twistor $A_{\alpha \beta}$, which therefore has the structure

$$
A_{\alpha \beta}=\left(\begin{array}{cc}
0 & P_{A}^{B^{\prime}} \\
P^{A^{\prime}}{ }_{B} 2 \mathrm{i} \bar{J}^{A^{\prime} B^{\prime}}
\end{array}\right) .
$$

Thus the quasi-local energy-momentum and self-dual angular momentum of the source are certain spinor parts of the kinematical twistor. In contrast to the ten complex components of a general symmetric twistor, it has only ten real components as a consequence of its structure (its spinor part $A_{A B}$ is identically zero) and the reality of $P^{A A^{\prime}}$. These properties can be reformulated by the infinity twistor and the Hermitian metric as conditions on $A_{\alpha \beta}$ : The vanishing of the spinor part $A_{A B}$ is equivalent to $A_{\alpha \beta} I^{\alpha \gamma} I^{\beta \delta}=0$ and the energy momentum is the $A_{\alpha \beta} Z^{\alpha} I^{\beta \gamma} H_{\gamma \gamma^{\prime}} \bar{Z}^{\gamma^{\prime}}$ part of the kinematical twistor, while the whole reality condition (ensuring both $A_{A B}=0$ and the reality of the energy-momentum) is equivalent to

$$
A_{\alpha \beta} I^{\beta \gamma} H_{\gamma \delta^{\prime}}=\bar{A}_{\delta^{\prime} \beta^{\prime}} \bar{I}^{\beta^{\prime} \gamma^{\prime}} H_{\gamma^{\prime} \alpha}
$$

Using the conjugate twistors this can be rewritten (and, in fact, usually it is written) as $A_{\alpha \beta} I^{\beta \gamma}=$ $\left(H^{\gamma \alpha^{\prime}} \bar{A}_{\alpha^{\prime} \beta^{\prime}} H^{\beta^{\prime} \delta}\right)\left(H_{\delta \delta^{\prime}} \bar{I}^{\delta^{\prime} \gamma^{\prime}} H_{\gamma^{\prime} \alpha}\right)=\bar{A}^{\gamma \delta} I_{\delta \alpha}$. Finally, the quasi-local mass can also be expressed by the kinematical twistor as its Hermitian norm [307] or as its determinant [371]:

$$
\begin{aligned}
& m^{2}=-P_{A} A^{\prime} P_{A^{\prime}}^{A}=-\frac{1}{2} A_{\alpha \beta} \bar{A}_{\alpha^{\prime} \beta^{\prime}} H^{\alpha \alpha^{\prime}} H^{\beta \beta^{\prime}}=-\frac{1}{2} A_{\alpha \beta} \bar{A}^{\alpha \beta}, \\
& m^{4}=4 \operatorname{det} A_{\alpha \beta}=\frac{1}{3 !} \varepsilon^{\alpha \beta \gamma \delta} \varepsilon^{\mu \nu \rho \sigma} A_{\alpha \mu} A_{\beta \nu} A_{\gamma \rho} A_{\delta \sigma} .
\end{aligned}
$$

Thus, to summarize, the various spinor parts of the kinematical twistor $A_{\alpha \beta}$ are the energymomentum and s.d. angular momentum. However, additional structures, namely the infinity twistor and the Hermitian scalar product, were needed to be able to 'isolate' its energy-momentum and angular momentum parts, and, in particular, to define the mass. Furthermore, the Hermiticity condition ensuring $A_{\alpha \beta}$ to have the correct number of components (ten reals) were also formulated in terms of these additional structures. 


\subsection{The original construction for curved spacetimes}

\subsubsection{2-surface twistors and the kinematical twistor}

In general spacetimes the twistor equations have only the trivial solution. Thus to be able to associate a kinematical twistor to a closed orientable spacelike 2 -surface $\mathcal{S}$ in general, the conditions on the spinor field $\omega^{A B}$ had to be relaxed. Penrose's suggestion [307, 308] is to consider $\omega^{A B}$ in Equation (43) to be the symmetrized product $\lambda^{(A} \omega^{B)}$ of spinor fields that are solutions of the 'tangential projection to $\mathcal{S}$ ' of the valence 1 twistor equation, the so-called 2-surface twistor equation. (The equation obtained as the 'tangential projection to $\mathcal{S}$ ' of the valence 2 twistor equation (46) would be under-determined [308].) Thus the quasi-local quantities are searched for in the form of a charge integral of the curvature:

$$
\begin{aligned}
A_{\mathcal{S}}[\lambda, \omega] & :=\frac{\mathrm{i}}{8 \pi G} \oint_{\mathcal{S}} \lambda^{A} \omega^{B} R_{A B c d}= \\
& =\frac{1}{4 \pi G} \oint_{\mathcal{S}}\left[\lambda^{0} \omega^{0}\left(\phi_{01}-\psi_{1}\right)+\left(\lambda^{0} \omega^{1}+\lambda^{1} \omega^{0}\right)\left(\phi_{11}+\Lambda-\psi_{2}\right)+\lambda^{1} \omega^{1}\left(\phi_{21}-\psi_{3}\right)\right] d \mathcal{S},
\end{aligned}
$$

where the second expression is given in the GHP formalism with respect to some GHP spin frame adapted to the 2-surface $\mathcal{S}$. Since the indices $c$ and $d$ on the right of the first expression are tangential to $\mathcal{S}$, this is just the charge integral of $F_{A B c d}$ in the spinor identity (24) of Section 4.1.5.

The 2-surface twistor equation that the spinor fields should satisfy is just the covariant spinor equation $\mathcal{T}_{E^{\prime} E A}{ }^{B} \lambda_{B}=0$. By Equation (25) its GHP form is $\mathcal{T} \lambda:=\left(\mathcal{T}^{+} \oplus \mathcal{T}^{-}\right) \lambda=0$, which is a first order elliptic system, and its index is $4(1-g)$, where $g$ is the genus of $\mathcal{S}$ [43]. Thus there are at least four (and in the generic case precisely four) linearly independent solutions to $\mathcal{T} \lambda=0$ on topological 2-spheres. However, there are 'exceptional' 2-spheres for which there exist at least five linearly independent solutions [221]. For such 'exceptional' 2-spheres (and for higher genus 2 -surfaces for which the twistor equation has only the trivial solution in general) the subsequent construction does not work. (The concept of quasi-local charges in Yang-Mills theory can also be introduced in an analogous way [370]). The space $\mathbf{T}_{\mathcal{S}}^{\alpha}$ of the solutions to $\mathcal{T}_{E^{\prime} E A}{ }^{B} \lambda_{B}=0$ is called the 2-surface twistor space. In fact, in the generic case this space is 4-complex-dimensional, and under conformal rescaling the pair $Z^{\alpha}=\left(\lambda^{A}, \mathrm{i} \Delta_{A^{\prime} A} \lambda^{A}\right)$ transforms like a valence 1 contravariant twistor. $Z^{\alpha}$ is called a 2 -surface twistor determined by $\lambda^{A}$. If $\mathcal{S}^{\prime}$ is another generic 2 -surface with the corresponding 2-surface twistor space $\mathbf{T}_{\mathcal{S}^{\prime}}^{\alpha}$, then although $\mathbf{T}_{\mathcal{S}}^{\alpha}$ and $\mathbf{T}_{\mathcal{S}^{\prime}}^{\alpha}$ are isomorphic as vector spaces, there is no canonical isomorphism between them. The kinematical twistor $A_{\alpha \beta}$ is defined to be the symmetric twistor determined by $A_{\alpha \beta} Z^{\alpha} W^{\beta}:=A_{\mathcal{S}}[\lambda, \omega]$ for any $Z^{\alpha}=\left(\lambda^{A}, \mathrm{i} \Delta_{A^{\prime} A^{A} \lambda^{A}}\right)$ and $W^{\alpha}=\left(\omega^{A}, \mathrm{i} \Delta_{A^{\prime} A} \omega^{A}\right)$ from $\mathbf{T}_{\mathcal{S}}^{\alpha}$. Note that $A_{\mathcal{S}}[\lambda, \omega]$ is constructed only from the 2-surface data on $\mathcal{S}$.

\subsubsection{The Hamiltonian interpretation of the kinematical twistor}

For the solutions $\lambda^{A}$ and $\omega^{A}$ of the 2-surface twistor equation, the spinor identity (24) reduces to Tod's expression [307, 313, 377] for the kinematical twistor, making it possible to re-express $A_{\mathcal{S}}[\lambda, \omega]$ by the integral of the Nester-Witten 2-form [356]. Indeed, if

$$
H_{\mathcal{S}}[\lambda, \bar{\mu}]:=\frac{1}{4 \pi G} \oint_{\mathcal{S}} u(\lambda, \bar{\mu})_{a b}=-\frac{1}{4 \pi G} \oint_{\mathcal{S}} \bar{\gamma}^{A^{\prime} B^{\prime}} \bar{\mu}_{A^{\prime}} \Delta_{B^{\prime} B} \lambda^{B} d \mathcal{S},
$$

then with the choice $\bar{\mu}_{A^{\prime}}:=\Delta_{A^{\prime} A \omega^{A}}$ this gives Penrose's charge integral by Equation (24): $A_{\mathcal{S}}[\lambda, \omega]=H_{\mathcal{S}}[\lambda, \bar{\mu}]$. Then, extending the spinor fields $\lambda^{A}$ and $\omega^{A}$ from $\mathcal{S}$ to a spacelike hypersurface $\Sigma$ with boundary $\mathcal{S}$ in an arbitrary way, by the Sparling equation it is straightforward to rewrite $A_{\mathcal{S}}[\lambda, \omega]$ in the form of the integral of the energy-momentum tensor of the matter fields

Living Reviews in Relativity

http: //www . livingreviews . org//rr-2004-4 
and the Sparling form on $\Sigma$. Since such an integral of the Sparling form can be interpreted as the Hamiltonian of general relativity, this is a quick re-derivation of Mason's [265, 266] Hamiltonian interpretation of Penrose's kinematical twistor: $A_{\mathcal{S}}[\lambda, \omega]$ is just the boundary term in the total Hamiltonian of the matter + gravity system, and the spinor fields $\lambda^{A}$ and $\omega^{A}$ (together with their 'projection parts' i $\Delta_{A^{\prime} A \lambda^{A}}$ and i $\left.\Delta_{A^{\prime} A} \omega^{A}\right)$ on $\mathcal{S}$ are interpreted as the spinor constituents of the special lapse and shift, the so-called 'quasi-translations' and 'quasi-rotations' of the 2-surface, on the 2 -surface itself.

\subsubsection{The Hermitian scalar product and the infinity twistor}

In general the natural pointwise Hermitian scalar product, defined by $\langle Z, \bar{W}\rangle:=-\mathrm{i}\left(\lambda^{A} \Delta_{A A^{\prime}} \bar{\omega}^{A^{\prime}}-\right.$ $\left.\bar{\omega}^{A^{\prime}} \Delta_{A A^{\prime}} \lambda^{A}\right)$, is not constant on $\mathcal{S}$, thus it does not define a Hermitian scalar product on the 2surface twistor space. As is shown in [220, 223,375], $\langle Z, \bar{W}\rangle$ is constant on $\mathcal{S}$ for any two 2 -surface twistors if and only if $\mathcal{S}$ can be embedded, at least locally, into some conformal Minkowski spacetime with its intrinsic metric and extrinsic curvatures. Such 2-surfaces are called non-contorted, while those that cannot be embedded are called contorted. One natural candidate for the Hermitian metric could be the average of $\langle Z, \bar{W}\rangle$ on $\mathcal{S}$ [307]: $H_{\alpha \beta^{\prime}} Z^{\alpha} \bar{W}^{\beta^{\prime}}:=[\operatorname{Area}(\mathcal{S})]^{-\frac{1}{2}} \oint_{\mathcal{S}}\langle Z, \bar{W}\rangle d \mathcal{S}$, which reduces to $\langle Z, \bar{W}\rangle$ on non-contorted 2-surfaces. Interestingly enough, $\oint_{\mathcal{S}}\langle Z, \bar{W}\rangle d \mathcal{S}$ can also be reexpressed by the integral (55) of the Nester-Witten 2-form [356]. Unfortunately, however, neither this metric nor the other suggestions appearing in the literature are conformally invariant. Thus, for contorted 2-surfaces, the definition of the quasi-local mass as the norm of the kinematical twistor (cf. Equation (52)) is ambiguous unless a natural $H_{\alpha \beta^{\prime}}$ is found.

If $\mathcal{S}$ is non-contorted, then the scalar product $\langle Z, \bar{W}\rangle$ defines the totally anti-symmetric twistor $\varepsilon_{\alpha \beta \gamma \delta}$, and for the four independent 2-surface twistors $Z_{1}^{\alpha}, \ldots, Z_{4}^{\alpha}$ the contraction $\varepsilon_{\alpha \beta \gamma \delta} Z_{1}^{\alpha} Z_{2}^{\beta} Z_{3}^{\gamma} Z_{4}^{\delta}$, and hence by Equation (49) the determinant $\nu$, is constant on $\mathcal{S}$. Nevertheless, $\nu$ can be constant even for contorted 2-surfaces for which $\langle Z, \bar{W}\rangle$ is not. Thus, the totally anti-symmetric twistor $\varepsilon_{\alpha \beta \gamma \delta}$ can exist even for certain contorted 2-surfaces. Therefore, an alternative definition of the quasi-local mass might be based on Equation (53) [371]. However, although the two mass definitions are equivalent in the linearized theory, they are different invariants of the kinematical twistor even in de Sitter or anti-de-Sitter spacetimes. Thus, if needed, the former notion of mass will be called the norm-mass, the latter the determinant-mass (denoted by $m_{\mathrm{D}}$ ).

If we want to have not only the notion of the mass but its reality is also expected, then we should ensure the Hermiticity of the kinematical twistor. But to formulate the Hermiticity condition (51), one also needs the infinity twistor. However, $-\varepsilon^{A^{\prime} B^{\prime}} \Delta_{A^{\prime} A} \lambda^{A} \Delta_{B^{\prime} B} \omega^{B}$ is not constant on $\mathcal{S}$ even if it is non-contorted, thus in general it does not define any twistor on $\mathbf{T}_{\mathcal{S}}^{\alpha}$. One might take its average on $\mathcal{S}$ (which can also be re-expressed by the integral of the Nester-Witten 2form [356]), but the resulting twistor would not be simple. In fact, even on 2-surfaces in de Sitter and anti-de Sitter spacetimes with cosmological constant $\lambda$ the natural definition for $I_{\alpha \beta}$ is $I_{\alpha \beta}:=$ $\operatorname{diag}\left(\lambda \varepsilon_{A B}, \varepsilon^{A^{\prime} B^{\prime}}\right)[313,311,371]$, while on round spheres in spherically symmetric spacetimes it

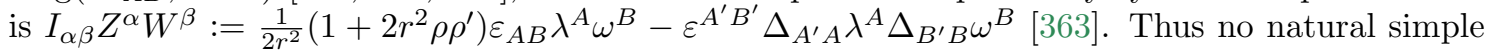
infinity twistor has been found in curved spacetime. Indeed, Helfer claims that no such infinity twistor can exist [197]: Even if the spacetime is conformally flat (whenever the Hermitian metric exists) the Hermiticity condition would be fifteen algebraic equations for the (at most) twelve real components of the 'would be' infinity twistor. Then, since the possible kinematical twistors form an open set in the space of symmetric twistors, the Hermiticity condition cannot be satisfied even for non-simple $I^{\alpha \beta}$ s. However, in contrast to the linearized gravity case, the infinity twistor should not be given once and for all on some 'universal' twistor space, that may depend on the actual gravitational field. In fact, the 2-surface twistor space itself depends on the geometry of $\mathcal{S}$, and hence all the structures thereon also.

Since in the Hermiticity condition (51) only the special combination $H^{\alpha}{ }_{\beta^{\prime}}:=I^{\alpha \beta} H_{\beta \beta^{\prime}}$ of the 
infinity and metric twistors (the so-called 'bar-hook' combination) appears, it might still be hoped that an appropriate $H^{\alpha}{ }_{\beta^{\prime}}$ could be found for a class of 2-surfaces in a natural way [377]. However, as far as the present author is aware of, no real progress has been achieved in this way.

\subsubsection{The various limits}

Obviously, the kinematical twistor vanishes in flat spacetime and, since the basic idea came from the linearized gravity, the construction gives the correct results in the weak field approximation. The nonrelativistic weak field approximation, i.e. the Newtonian limit, was clarified by Jeffryes [222]. He considers a 1-parameter family of spacetimes with perfect fluid source such that in the $\lambda \rightarrow 0$ limit of the parameter $\lambda$ one gets a Newtonian spacetime, and, in the same limit, the 2-surface $\mathcal{S}$ lies in a $t=$ const. hypersurface of the Newtonian time $t$. In this limit the pointwise Hermitian scalar product is constant, and the norm-mass can be calculated. As could be expected, for the leading $\lambda^{2}$ order term in the expansion of $m$ as a series of $\lambda$ he obtained the conserved Newtonian mass. The Newtonian energy, including the kinetic and the Newtonian potential energy, appears as a $\lambda^{4}$ order correction.

The Penrose definition for the energy-momentum and angular momentum can be applied to the cuts $\mathcal{S}$ of the future null infinity $\mathscr{I}^{+}$of an asymptotically flat spacetime [307, 313]. Then every element of the construction is built from conformally rescaled quantities of the non-physical spacetime. Since $\mathscr{I}^{+}$is shear-free, the 2 -surface twistor equations on $\mathcal{S}$ decouple, and hence the solution space admits a natural infinity twistor $I_{\alpha \beta}$. It singles out precisely those solutions whose primary spinor parts span the asymptotic spin space of Bramson (see Section 4.2.4), and they will be the generators of the energy-momentum. Although $\mathcal{S}$ is contorted, and hence there is no natural Hermitian scalar product, there is a twistor $H^{\alpha}{ }_{\beta^{\prime}}$ with respect to which $A_{\alpha \beta}$ is Hermitian. Furthermore, the determinant $\nu$ is constant on $\mathcal{S}$, and hence it defines a volume 4 -form on the 2 -surface twistor space [377]. The energy-momentum coming from $A_{\alpha \beta}$ is just that of Bondi and Sachs. The angular momentum defined by $A_{\alpha \beta}$ is, however, new. It has a number of attractive properties. First, in contrast to definitions based on the Komar expression, it does not have the 'factor-of-two anomaly' between the angular momentum and the energy-momentum. Since its definition is based on the solutions of the 2-surface twistor equations (which can be interpreted as the spinor constituents of certain BMS vector fields generating boost-rotations) instead of the BMS vector fields themselves, it is free of supertranslation ambiguities. In fact, the 2-surface twistor space on $\mathcal{S}$ reduces the BMS Lie algebra to one of its Poincaré subalgebras. Thus the concept of the 'translation of the origin' is moved from null infinity to the twistor space (appearing in the form of a 4-parameter family of ambiguities in the potential for the shear $\sigma$ ), and the angular momentum transforms just in the expected way under such a 'translation of the origin'. As was shown in [129], Penrose's angular momentum can be considered as a supertranslation of previous definitions. The corresponding angular momentum flux through a portion of the null infinity between two cuts was calculated in $[129,196]$ and it was shown that this is precisely that given by Ashtekar and Streubel [29] (see also [336, 337, 128]).

The other way of determining the null infinity limit of the energy-momentum and angular momentum is to calculate them for the large spheres from the physical data, instead of the spheres at null infinity from the conformally rescaled data. These calculations were done by Shaw [338, 340]. At this point it should be noted that the $r \rightarrow \infty$ limit of $A_{\alpha \beta}$ vanishes, and it is $\sqrt{\operatorname{Area}\left(\mathcal{S}_{r}\right)} A_{\alpha \beta}$ that yields the energy-momentum and angular momentum at infinity (see the remarks following Equation (15)). The specific radiative solution for which the Penrose mass has been calculated is that of Robinson and Trautman [371]. The 2-surfaces for which the mass was calculated are the $r=$ const. cuts of the geometrically distinguished outgoing null hypersurfaces $u=$ const. Tod found that, for given $u$, the mass $m$ is independent of $r$, as could be expected because of the lack of the incoming radiation.

Living Reviews in Relativity

http: //www. livingreviews . org//rr-2004-4 
The large sphere limit of the 2-surface twistor space and the Penrose construction were investigated by Shaw in the Sommers [344], the Ashtekar-Hansen [23], and the Beig-Schmidt [48] models of spatial infinity in $[334,335,337]$. Since no gravitational radiation is present near the spatial infinity, the large spheres are (asymptotically) non-contorted, and both the Hermitian scalar product and the infinity twistor are well-defined. Thus the energy-momentum and angular momentum (and, in particular, the mass) can be calculated. In vacuum he recovered the Ashtekar-Hansen expression for the energy-momentum and angular momentum, and proved their conservation if the Weyl curvature is asymptotically purely electric. In the presence of matter the conservation of the angular momentum was investigated in [339].

The Penrose mass in asymptotically anti-de-Sitter spacetimes was studied by Kelly [234]. He calculated the kinematical twistor for spacelike cuts $\mathcal{S}$ of the infinity $\mathscr{I}$, which is now a timelike 3 -manifold in the non-physical spacetime. Since $\mathscr{I}$ admits global 3 -surface twistors (see the next Section 7.2.5), $\mathcal{S}$ is non-contorted. In addition to the Hermitian scalar product there is a natural infinity twistor, and the kinematical twistor satisfies the corresponding Hermiticity condition. The energy-momentum 4-vector coming from the Penrose definition is shown to coincide with that of Ashtekar and Magnon [27]. Therefore, the energy-momentum 4-vector is future pointing and timelike if there is a spacelike hypersurface extending to $\mathscr{I}$ on which the dominant energy condition is satisfied. Consequently, $m^{2} \geq 0$. Kelly showed that $m_{\mathrm{D}}^{2}$ is also non-negative and in vacuum it coincides with $m^{2}$. In fact [377], $m \geq m_{\mathrm{D}} \geq 0$ holds.

\subsubsection{The quasi-local mass of specific 2-surfaces}

The Penrose mass has been calculated in a large number of specific situations. Round spheres are always non-contorted [375], thus the norm-mass can be calculated. (In fact, axi-symmetric 2-surfaces in spacetimes with twist-free rotational Killing vector are non-contorted [223].) The Penrose mass for round spheres reduces to the standard energy expression discussed in Section 4.2.1 [371]. Thus every statement given in Section 4.2.1 for round spheres is valid for the Penrose mass, and we do not repeat them. In particular, for round spheres in a $t=$ const. slice of the Kantowski-Sachs spacetime this mass is independent of the 2-surfaces [368]. Interestingly enough, although these spheres cannot be shrunk to a point (thus the mass cannot be interpreted as 'the 3-volume integral of some mass density'), the time derivative of the Penrose mass looks like the mass conservation equation: It is minus the pressure times the rate of change of the 3-volume of a sphere in flat space with the same area as $\mathcal{S}$ [376]. In conformally flat spacetimes [371] the 2-surface twistors are just the global twistors restricted to $\mathcal{S}$, and the Hermitian scalar product is constant on $\mathcal{S}$. Thus the norm-mass is well-defined.

The construction works nicely even if global twistors exist only on a (say) spacelike hypersurface $\Sigma$ containing $\mathcal{S}$. These twistors are the so-called 3 -surface twistors [371, 373], which are solutions of certain (overdetermined) elliptic partial differential equations, the so-called 3-surface twistor equations, on $\Sigma$. These equations are completely integrable (i.e. they admit the maximal number of linearly independent solutions, namely four) if and only if $\Sigma$ with its intrinsic metric and extrinsic curvature can be embedded, at least locally, into some conformally flat spacetime [373]. Such hypersurfaces are called non-contorted. It might be interesting to note that the non-contorted hypersurfaces can also be characterized as the critical points of the Chern-Simons functional built from the real Sen connection on the Lorentzian vector bundle or from the 3-surface twistor connection on the twistor bundle over $\Sigma[49,361]$. Returning to the quasi-local mass calculations, Tod showed that in vacuum the kinematical twistor for a 2-surface $\mathcal{S}$ in a non-contorted $\Sigma$ depends only on the homology class of $\mathcal{S}$. In particular, if $\mathcal{S}$ can be shrunk to a point then the corresponding kinematical twistor is vanishing. Since $\Sigma$ is non-contorted, $\mathcal{S}$ is also non-contorted, and hence the norm-mass is well-defined. This implies that the Penrose mass in the Schwarzschild solution is the Schwarzschild mass for any non-contorted 2-surface that can be deformed into a round sphere, and 
it is zero for those that do not link the black hole [375]. Thus, in particular, the Penrose mass can be zero even in curved spacetimes.

A particularly interesting class of non-contorted hypersurfaces is that of the conformally flat time-symmetric initial data sets. Tod considered Wheeler's solution of the time-symmetric vacuum constraints describing $n$ 'points at infinity' (or, in other words, $n-1$ black holes) and 2-surfaces in such a hypersurface [371]. He found that the mass is zero if $\mathcal{S}$ does not link any black hole, it is the mass $M_{i}$ of the $i$-th black hole if $\mathcal{S}$ links precisely the $i$-th hole, it is $M_{i}+M_{j}-M_{i} M_{j} / d_{i j}+\mathcal{O}\left(1 / d_{i j}^{2}\right)$ if $\mathcal{S}$ links precisely the $i$-th and the $j$-th holes, where $d_{i j}$ is some appropriate measure of the distance of the holes, ..., etc. Thus, the mass of the $i$-th and $j$-th holes as a single object is less than the sum of the individual masses, in complete agreement with our physical intuition that the potential energy of the composite system should contribute to the total energy with negative sign.

Beig studied the general conformally flat time-symmetric initial data sets describing $n$ 'points at infinity' [45]. He found a symmetric trace-free and divergence-free tensor field $T^{a b}$ and, for any conformal Killing vector $\xi^{a}$ of the data set, defined the 2 -surface flux integral $P(\xi)$ of $T^{a b} \xi_{b}$ on $\mathcal{S}$. He showed that $P(\xi)$ is conformally invariant, depends only on the homology class of $\mathcal{S}$, and, apart from numerical coefficients, for the ten (locally existing) conformal Killing vectors these are just the components of the kinematical twistor derived by Tod in [371] (and discussed in the previous paragraph). In particular, Penrose's mass in Beig's approach is proportional to the length of the $P$ 's with respect to the Cartan-Killing metric of the conformal group of the hypersurface.

Tod calculated the quasi-local mass for a large class of axi-symmetric 2-surfaces (cylinders) in various LRS Bianchi and Kantowski-Sachs cosmological models [376] and more general cylindrically symmetric spacetimes [378]. In all these cases the 2-surfaces are non-contorted, and the construction works. A technically interesting feature of these calculations is that the 2-surfaces have edges, i.e. they are not smooth submanifolds. The twistor equation is solved on the three smooth pieces of the cylinder separately, and the resulting spinor fields are required to be continuous at the edges. This matching reduces the number of linearly independent solutions to four. The projection parts of the resulting twistors, the $\mathrm{i} \Delta_{A^{\prime} A_{A}} \lambda^{A} \mathrm{~s}$, are not continuous at the edges. It turns out that the cylinders can be classified invariantly to be hyperbolic, parabolic, or elliptic. Then the structure of the quasi-local mass expressions is not simply 'density' $\times$ 'volume', but they are proportional to a 'type factor' $f(L)$ as well, where $L$ is the coordinate length of the cylinder. In the hyperbolic, parabolic, and elliptic cases this factor is $\sinh \omega L /(\omega L), 1$, and $\sin \omega L /(\omega L)$, respectively, where $\omega$ is an invariant of the cylinder. The various types are interpreted as the presence of a positive, zero, or negative potential energy. In the elliptic case the mass may be zero for finite cylinders. On the other hand, for static perfect fluid spacetimes (hyperbolic case) the quasi-local mass is positive. A particularly interesting spacetime is that describing cylindrical gravitational waves, whose presence is detected by the Penrose mass. In all these cases the determinant-mass has also been calculated and found to coincide with the norm-mass. A numerical investigation of the axi-symmetric Brill waves on the Schwarzschild background was presented in [69]. It was found that the quasi-local mass is positive, and it is very sensitive to the presence of the gravitational waves.

Another interesting issue is the Penrose inequality for black holes (see Section 13.2.1). Tod showed $[374,375]$ that for static black holes the Penrose inequality holds if the mass of the hole is defined to be the Penrose quasi-local mass of the spacelike cross section $\mathcal{S}$ of the event horizon. The trick here is that $\mathcal{S}$ is totally geodesic and conformal to the unit sphere, and hence it is noncontorted and the Penrose mass is well-defined. Then the Penrose inequality will be a Sobolev-type inequality for a non-negative function on the unit sphere. This inequality was tested numerically in [69].

Apart from the cuts of $\mathscr{I}^{+}$in radiative spacetimes, all the 2-surfaces discussed so far were non-contorted. The spacelike cross section of the event horizon of the Kerr black hole provides a contorted 2-surface [377]. Thus although the kinematical twistor can be calculated for this, the

Living Reviews in Relativity

http: //www. livingreviews . org//rr-2004-4 
construction in its original form cannot yield any mass expression. The original construction has to be modified.

\subsubsection{Small surfaces}

The properties of the Penrose construction that we have discussed are very remarkable and promising. However, the small surface calculations showed clearly some unwanted feature of the original construction [372, 235, 398], and forced its modification.

First, although the small spheres are contorted in general, the leading term of the pointwise Hermitian scalar product is constant: $\lambda^{A} \Delta_{A A^{\prime}} \bar{\omega}^{A^{\prime}}-\bar{\omega}^{A^{\prime}} \Delta_{A^{\prime} A} \lambda^{A}=$ const. $+\mathcal{O}(r)$ for any 2-surface twistors $Z^{\alpha}=\left(\lambda^{A}, \mathrm{i} \Delta_{A^{\prime} A} \lambda^{A}\right)$ and $W^{\alpha}=\left(\omega^{A}, \mathrm{i} \Delta_{A^{\prime} A} \omega^{A}\right)$ [372, 235]. Since in non-vacuum spacetimes the kinematical twistor has only the '4-momentum part' in the leading $\mathcal{O}\left(r^{3}\right)$ order with $P_{a}=\frac{4 \pi}{3} r^{3} T_{a b} t^{b}$, the Penrose mass, calculated with the norm above, is just the expected mass in the leading $\mathcal{O}\left(r^{3}\right)$ order. Thus it is positive if the dominant energy condition is satisfied. On the other hand, in vacuum the structure of the kinematical twistor is

$$
A_{\alpha \beta}=\left(\begin{array}{cc}
2 \mathrm{i} \lambda_{A B} & P_{A} B^{\prime} \\
P_{B}^{A^{\prime}} & 0
\end{array}\right)+\mathcal{O}\left(r^{6}\right),
$$

where $\lambda_{A B}=\mathcal{O}\left(r^{5}\right)$ and $P_{A A^{\prime}}=\frac{2}{45 G} r^{5} \psi_{A B C D} \bar{\chi}_{A^{\prime} B^{\prime} C^{\prime} D^{\prime}} t^{B B^{\prime}} t^{C C^{\prime}} t^{D D^{\prime}}$ with $\chi_{A B C D}:=\psi_{A B C D}-$

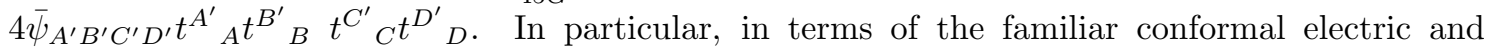
magnetic parts of the curvature the leading term in the time component of the 4-momentum is $P_{A A^{\prime}} t^{A A^{\prime}}=\frac{1}{45 G} H_{a b}\left(H^{a b}-\mathrm{i} E^{a b}\right)$. Then the corresponding norm-mass, in the leading order, can even be complex! For an $\mathcal{S}_{r}$ in the $t=$ const. hypersurface of the Schwarzschild spacetime this is zero (as it must be in the light of the results of the previous Section 7.2.5, because this is a non-contorted spacelike hypersurface), but for a general small 2-sphere not lying in such a hypersurface $P_{A A^{\prime}}$ is real and spacelike, and hence $m^{2}<0$. In the Kerr spacetime $P_{A A^{\prime}}$ itself is complex [372, 235].

\subsection{The modified constructions}

Independently of the results of the small sphere calculations, Penrose claimed that in the Schwarzschild spacetime the quasi-local mass expression should yield the same zero value on 2-surfaces, contorted or not, which do not surround the black hole. (For the motivations and the arguments, see [309].) Thus the original construction should be modified, and the negative results for the small spheres above strengthened this need. A much more detailed review of the various modifications is given by Tod in [377].

\subsubsection{The 'improved' construction with the determinant}

A careful analysis of the roots of the difficulties lead Penrose [309, 313] (see also [372, 235, 377]) to suggest the modified definition for the kinematical twistor

$$
A_{\alpha \beta}^{\prime} Z^{\alpha} W^{\beta}:=\frac{\mathrm{i}}{8 \pi G} \oint_{\mathcal{S}} \eta \lambda^{A} \omega^{B} R_{A B c d},
$$

where $\eta$ is a constant multiple of the determinant $\nu$ in Equation (49). Since on non-contorted 2 -surfaces the determinant $\nu$ is constant, for such surfaces $A_{\alpha \beta}^{\prime}$ reduces to $A_{\alpha \beta}$, and hence all the nice properties proven for the original construction on non-contorted 2-surfaces are shared by $A_{\alpha \beta}^{\prime}$ too. The quasi-local mass calculated from Equation (57) for small spheres (in fact, for small ellipsoids [235]) in vacuum is vanishing in the fifth order. Thus, apparently, the difficulties have 
been resolved. However, as Woodhouse pointed out, there is an essential ambiguity in the (nonvanishing, sixth order) quasi-local mass [398]. In fact, the structure of the modified kinematical twistor has the form (56) with vanishing $P_{B}^{A^{\prime}}$ and $P_{A}{ }^{B^{\prime}}$ but with non-vanishing $\lambda_{A B}$ in the fifth order. Then in the quasi-local mass (in the leading sixth order) there will be a term coming from the (presumably non-vanishing) sixth order part of $P^{A^{\prime}}{ }_{B}$ and $P_{A} B^{\prime}$ and the constant part of the Hermitian scalar product, and the fifth order $\lambda_{A B}$ and the still ambiguous $\mathcal{O}(r)$ order part of the Hermitian metric.

\subsubsection{Modification through Tod's expression}

These anomalies lead Penrose to modify $A_{\alpha \beta}^{\prime}$ slightly [310]. This modified form is based on Tod's form of the kinematical twistor:

$$
A_{\alpha \beta}^{\prime \prime} Z^{\alpha} W^{\beta}:=\frac{1}{4 \pi G} \oint_{\mathcal{S}} \bar{\gamma}^{A^{\prime} B^{\prime}}\left[\mathrm{i} \Delta_{A^{\prime} A}\left(\sqrt{\eta} \lambda^{A}\right)\right]\left[\mathrm{i} \Delta_{B^{\prime} B}\left(\sqrt{\eta} \omega^{B}\right)\right] d \mathcal{S} .
$$

The quasi-local mass on small spheres coming from $A_{\alpha \beta}^{\prime \prime}$ is positive [377].

\subsubsection{Mason's suggestions}

A beautiful property of the original construction was its connection with the Hamiltonian formulation of the theory [265]. Unfortunately, such a simple Hamiltonian interpretation is lacking for the modified constructions. Although the form of Equation (58) is that of the integral of the Nester-Witten 2-form, and the spinor fields $\sqrt{\eta} \lambda^{A}$ and i $\Delta_{A^{\prime} A}\left(\sqrt{\eta} \lambda^{A}\right)$ could still be considered as the spinor constituents of the 'quasi-Killing vectors' of the 2-surface $\mathcal{S}$, their structure is not so simple because the factor $\eta$ itself depends on all of the four independent solutions of the 2-surface twistor equation in a rather complicated way.

To have a simple Hamiltonian interpretation Mason suggested further modifications [265, 266]. He considers the four solutions $\lambda_{i}^{A}, i=1, \ldots, 4$, of the 2-surface twistor equations, and uses these solutions in the integral (55) of the Nester-Witten 2-form. Since $H_{\mathcal{S}}$ is a Hermitian bilinear form on the space of the spinor fields (see Section 8 below), he obtains 16 real quantities as the components of the $4 \times 4$ Hermitian matrix $E_{i j}:=H_{\mathcal{S}}\left[\lambda_{i}, \bar{\lambda}_{j}\right]$. However, it is not clear how the four 'quasi-translations' of $\mathcal{S}$ should be found among the 16 vector fields $\lambda_{i}^{A} \bar{\lambda}_{j}^{A^{\prime}}$ (called 'quasi-conformal Killing vectors' of $\mathcal{S}$ ) for which the corresponding quasi-local quantities could be considered as the quasi-local energy-momentum. Nevertheless, this suggestion leads us to the next class of quasi-local quantities.

Living Reviews in Relativity

http: //www. livingreviews. org/lrr-2004-4 


\section{Approaches Based on the Nester-Witten 2-Form}

We saw in Section 3.2 that

- both the ADM and Bondi-Sachs energy-momenta can be re-expressed by the integral of the Nester-Witten 2-form $u(\lambda, \bar{\mu})_{a b}$,

- the proof of the positivity of the ADM and Bondi-Sachs masses is relatively simple in terms of the 2-component spinors, and

- the integral of Møller's tetrad superpotential for the energy-momentum, coming from his tetrad Lagrangian (9), is just the integral of $u\left(\lambda \underline{A}, \bar{\lambda}^{\prime}\right)_{a b}$, where $\left\{\lambda \frac{A}{A}\right\}$ is a normalized spinor dyad.

Thus, both from conceptual and pragmatic points of views, it seems natural to search for the quasi-local energy-momentum in the form of the integral of the Nester-Witten 2-form. All the quasi-local energy-momenta based on the integral of the Nester-Witten 2-form have a natural Lagrangian interpretation [362]. Thus first let us discuss briefly the most important properties of such integrals.

If $\mathcal{S}$ is any closed, orientable spacelike 2 -surface and $\lambda_{A}, \mu_{A}$ are arbitrary spinor fields, then in the integral $H_{\mathcal{S}}[\lambda, \bar{\mu}]$, defined by Equation (55), only the tangential derivative of $\lambda_{A}$ appears. $\left(\mu_{A}\right.$ is involved in $H_{\mathcal{S}}[\lambda, \bar{\mu}]$ algebraically.) Thus, by Equation (13), $H_{\mathcal{S}}: C^{\infty}\left(\mathcal{S}, \mathbf{S}_{A}\right) \times C^{\infty}\left(\mathcal{S}, \mathbf{S}_{A}\right) \rightarrow \mathbb{C}$ is a Hermitian scalar product on the (infinite-dimensional complex) vector space of smooth spinor fields on $\mathcal{S}$. Thus, in particular, the spinor fields in $H_{\mathcal{S}}[\lambda, \bar{\mu}]$ need be defined only on $\mathcal{S}$, and $\overline{H_{\mathcal{S}}[\lambda, \bar{\mu}]}=H_{\mathcal{S}}[\mu, \bar{\lambda}]$ holds. A remarkable property of $H_{\mathcal{S}}$ is that if $\lambda_{A}$ is a constant spinor field on $\mathcal{S}$ with respect to the covariant derivative $\Delta_{e}$, then $H_{\mathcal{S}}[\lambda, \bar{\mu}]=0$ for any smooth spinor field $\mu_{A}$ on $\mathcal{S}$. Furthermore, if $\lambda_{A}^{A}=\left(\lambda_{A}^{0}, \lambda_{A}^{1}\right)$ is any pair of smooth spinor fields on $\mathcal{S}$, then for any constant $S L(2, \mathbb{C})$ matrix $\Lambda_{\underline{A}} \underline{B}$ one has $H_{\mathcal{S}}\left[\lambda^{\underline{C}} \Lambda_{\underline{C}} \underline{A}, \bar{\lambda}^{\underline{D}^{\prime}} \bar{\Lambda}_{\underline{D}^{\prime}} \underline{B}^{\prime}\right]=H_{\mathcal{S}}\left[\lambda \underline{C}, \bar{\lambda}^{\prime} \underline{\underline{D}}^{\prime}\right] \Lambda_{\underline{C}} \underline{A} \bar{\Lambda}_{\underline{D}^{\prime}} \underline{\underline{B}}^{\prime}$, i.e. the integrals $H_{\mathcal{S}}\left[\lambda \underline{A}, \bar{\lambda}^{\prime}{ }^{\prime}\right]$ transform as the spinor components of a real Lorentz vector over the two-complex dimensional space spanned by $\lambda_{A}^{0}$ and $\lambda_{A}^{1}$. Therefore, to have a well-defined quasilocal energy-momentum vector we have to specify some 2 -dimensional subspace $\mathbf{S} \underline{A}$ of the infinitedimensional space $C^{\infty}\left(\mathcal{S}, \mathbf{S}_{A}\right)$ and a symplectic metric $\varepsilon_{A} \underline{B}$ thereon. Thus underlined capital Roman indices will be referring to this space. The elements of this subspace would be interpreted as the spinor constituents of the 'quasi-translations' of the surface $\mathcal{S}$. Since in Møller's tetrad approach it is natural to choose the orthonormal vector basis to be a basis in which the translations have constant components (just as the constant orthonormal bases in Minkowski spacetime which are bases in the space of translations), the spinor fields $\lambda_{A}^{A}$ could also be interpreted as the spinor basis that should be used to construct the orthonormal vector basis in Møller's superpotential (10). In this sense the choice of the subspace $\mathbf{S} \underline{A}$ and the metric $\varepsilon_{\underline{A}} \underline{B}$ is just a gauge reduction, or a choice for the 'reference configuration' of Section 3.3.3.

Once the spin space $\left(\mathbf{S}^{A}, \varepsilon_{\underline{A}} \underline{B}\right)$ is chosen, the quasi-local energy-momentum is defined to be $P_{\mathcal{S}}^{\underline{A}} \underline{B}^{\prime}:=H_{\mathcal{S}}\left[\lambda \underline{A}, \bar{\lambda} \underline{B}^{\prime}\right]$ and the corresponding quasi-local mass $m_{\mathcal{S}}$ is $m_{\mathcal{S}}^{2}:=\varepsilon_{\underline{A}} \underline{B} \varepsilon_{\underline{A}^{\prime}} \underline{B}^{\prime} P_{\mathcal{S}} \underline{A}^{\prime} P_{\mathcal{S}}^{\underline{B}} \underline{B}^{\prime}$. In particular, if one of the spinor fields $\lambda_{A}^{A}$, e.g. $\lambda_{A}^{0}$, is constant on $\mathcal{S}$ (which means that the geometry of $\mathcal{S}$ is considerably restricted), then $P_{\mathcal{S}}^{00^{\prime}}=P_{\mathcal{S}}^{01^{\prime}}=P_{\mathcal{S}}^{10^{\prime}}=0$, and hence the corresponding mass $m_{\mathcal{S}}$ is zero. If both $\lambda_{A}^{0}$ and $\lambda_{A}^{1}$ are constant (in particular, when they are the restrictions to $\mathcal{S}$ of the two constant spinor fields in the Minkowski spacetime), then $P_{\mathcal{S}} \underline{\underline{B}} \underline{ }^{\prime}$ itself is vanishing.

Therefore, to summarize, the only thing that needs to be specified is the spin space $\left(\mathbf{S}^{\underline{A}}, \varepsilon_{\underline{A}} \underline{B}\right)$, and the various suggestions for the quasi-local energy-momentum based on the integral of the Nester-Witten 2-form correspond to the various choices for this spin space. 


\subsection{The Ludvigsen-Vickers construction}

\subsubsection{The definition}

Suppose that the spacetime is asymptotically flat at future null infinity, and the closed spacelike 2surface $\mathcal{S}$ can be joined to future null infinity by a smooth null hypersurface $\mathcal{N}$. Let $\mathcal{S}_{\infty}:=\mathcal{N} \cap \mathscr{I}^{+}$, the cut defined by the intersection of $\mathcal{N}$ with the future null infinity. Then the null geodesic generators of $\mathcal{N}$ define a smooth bijection between $\mathcal{S}$ and the cut $\mathcal{S}_{\infty}$ (and hence, in particular, $\mathcal{S} \approx S^{2}$ ). We saw in Section 4.2.4 that on the cut $\mathcal{S}_{\infty}$ at the future null infinity we have the asymptotic spin space $\left(\mathbf{S}_{\infty}^{A}, \varepsilon_{\underline{A}} \underline{B}\right)$. The suggestion of Ludvigsen and Vickers [259] for the spin space $\left(\mathbf{S}^{\underline{A}}, \varepsilon_{\underline{A}} \underline{B}\right)$ on $\mathcal{S}$ is to import the two independent solutions of the asymptotic twistor equations, i.e. the asymptotic spinors, from the future null infinity back to the 2-surface along the null geodesic generators of the null hypersurface $\mathcal{N}$. Their propagation equations, given both in terms of spinors and in the GHP formalism, are

$$
\begin{aligned}
& o^{A} \bar{o}^{A^{\prime}}\left(\nabla_{A A^{\prime}} \lambda_{B}\right) o^{B}=\mathrm{p} \lambda_{0}=0, \\
& \iota^{A} \bar{o}^{A^{\prime}}\left(\nabla_{A A^{\prime}} \lambda_{B}\right) o^{B}=\succ^{\prime} \lambda_{0}+\rho \lambda_{1}=0 .
\end{aligned}
$$

Here $\varepsilon_{\mathbf{A}}^{A}=\left\{o^{A}, \iota^{A}\right\}$ is the GHP spin frame introduced in Section 4.2.4, and by Equation (25) the second half of these equations is just $\Delta^{+} \lambda=0$. It should be noted that the choice $(59,60)$ for the propagation law of the spinors is 'natural' in the sense that in flat spacetime $(59,60)$ reduce to the condition of parallel propagation, and Equation (60) is just the appropriate part of the asymptotic twistor equation of Bramson. We call the spinor fields obtained by using Equations $(59,60)$ the Ludvigsen-Vickers spinors on $\mathcal{S}$. Thus, given an asymptotic spinor at infinity, we propagate its zero-th components (with respect to the basis $\varepsilon_{\mathbf{A}}^{A}$ ) to $\mathcal{S}$ by Equation (59). This will be the zeroth component of the Ludvigsen-Vickers spinor. Then its first component will be determined by Equation (60), provided $\rho$ is not vanishing on any open subset of $\mathcal{S}$. If $\lambda_{A}^{0}$ and $\lambda_{A}^{1}$ are LudvigsenVickers spinors on $\mathcal{S}$ obtained by Equations $(59,60)$ from two asymptotic spinors that formed a normalized spin frame, then, by considering $\lambda_{A}^{0}$ and $\lambda_{A}^{1}$ to be normalized in $\mathbf{S} \underline{A}$, we define the symplectic metric $\varepsilon_{\underline{A}} \underline{B}$ on $\mathbf{S}^{\underline{A}}$ to be that with respect to which $\lambda_{A}^{0}$ and $\lambda_{A}^{1}$ form a normalized spin frame. Note, however, that this symplectic metric is not connected with the symplectic fibre metric $\varepsilon_{A B}$ of the spinor bundle $\mathbf{S}^{A}(\mathcal{S})$ over $\mathcal{S}$. Indeed, in general, $\lambda \frac{A}{A} \lambda \frac{B}{B} \varepsilon^{A B}$ is not constant on $\mathcal{S}$, and hence $\varepsilon_{A B}$ does not determine any symplectic metric on the space $\mathbf{S}^{\underline{A}}$ of the Ludvigsen-Vickers spinors. In Minkowski spacetime the two Ludvigsen-Vickers spinors are just the restriction to $\mathcal{S}$ of the two constant spinors.

\subsubsection{Remarks on the validity of the construction}

Before discussing the usual questions about the properties of the construction (positivity, monotonicity, the various limits, etc.), we should make some general remarks. First, it is obvious that the Ludvigsen-Vickers energy-momentum in its form above cannot be defined in a spacetime which is not asymptotically flat at null infinity. Thus their construction is not genuinely quasi-local, because it depends not only on the (intrinsic and extrinsic) geometry of $\mathcal{S}$, but on the global structure of the spacetime as well. In addition, the requirement of the smoothness of the null hypersurface $\mathcal{N}$ connecting the 2 -surface to the null infinity is a very strong restriction. In fact, for general (even for convex) 2-surfaces in a general asymptotically flat spacetime conjugate points will develop along the (outgoing) null geodesics orthogonal to the 2-surface [304, 175]. Thus either the 2-surface must be near enough to the future null infinity (in the conformal picture), or the spacetime and the 2 -surface must be nearly spherically symmetric (or the former cannot be 'very much curved' and the latter cannot be 'very much bent').

This limitation yields that in general the original construction above does not have a small sphere limit. However, using the same propagation equations $(59,60)$ one could define a quasi-

Living Reviews in Relativity

http: //www. livingreviews.org/lrr-2004-4 
local energy-momentum for small spheres [259,66]. The basic idea is that there is a spin space at the vertex $p$ of the null cone in the spacetime whose spacelike cross section is the actual 2-surface, and the Ludvigsen-Vickers spinors on $\mathcal{S}$ are defined by propagating these spinors from the vertex $p$ to $\mathcal{S}$ via Equations $(59,60)$. This definition works in arbitrary spacetime, but the 2 -surface cannot be extended to a large sphere near the null infinity, and it is still not genuinely quasi-local.

\subsubsection{Monotonicity, mass-positivity and the various limits}

Once the Ludvigsen-Vickers spinors are given on a spacelike 2 -surface $\mathcal{S}_{r}$ of constant affine parameter $r$ in the outgoing null hypersurface $\mathcal{N}$, then they are uniquely determined on any other spacelike 2 -surface $\mathcal{S}_{r^{\prime}}$ in $\mathcal{N}$, too, i.e. the propagation law $(59,60)$ defines a natural isomorphism between the space of the Ludvigsen-Vickers spinors on different 2-surfaces of constant affine parameter in the same $\mathcal{N}$. ( $r$ need not be a Bondi-type coordinate.) This makes it possible to compare the components of the Ludvigsen-Vickers energy-momenta on different surfaces. In fact [259], if the dominant energy condition is satisfied (at least on $\mathcal{N}$ ), then for any Ludvigsen-Vickers spinor $\lambda^{A}$ and affine parameter values $r_{1} \leq r_{2}$ one has $H_{\mathcal{S}_{r_{1}}}[\lambda, \bar{\lambda}] \leq H_{\mathcal{S}_{r_{2}}}[\lambda, \bar{\lambda}]$, and the difference $H_{\mathcal{S}_{r_{2}}}[\lambda, \bar{\lambda}]-H_{\mathcal{S}_{r_{1}}}[\lambda, \bar{\lambda}] \geq 0$ can be interpreted as the energy flux of the matter and the gravitational radiation through $\mathcal{N}$ between $\mathcal{S}_{r_{1}}$ and $\mathcal{S}_{r_{2}}$. Thus both $P_{\mathcal{S}_{r}}^{00^{\prime}}$ and $P_{\mathcal{S}_{r}}^{11^{\prime}}$ are increasing with $r$ ('massgain'). A similar monotonicity property ('mass-loss') can be proven on ingoing null hypersurfaces, but then the propagation law $(59,60)$ should be replaced by $\mathrm{p}^{\prime} \lambda_{1}=0$ and $-\Delta^{-} \lambda:=\varnothing \lambda_{1}+\rho^{\prime} \lambda_{0}=0$. Using these equations the positivity of the Ludvigsen-Vickers mass was proven in various special cases in [259].

Concerning the positivity properties of the Ludvigsen-Vickers mass and energy, first it is obvious by the remarks on the nature of the propagation law $(59,60)$ that in Minkowski spacetime the Ludvigsen-Vickers energy-momentum is vanishing. However, in the proof of the non-negativity of the Dougan-Mason energy (discussed in Section 8.2) only the $\lambda_{A} \in \operatorname{ker} \Delta^{+}$part of the propagation equations is used. Therefore, as realized by Bergqvist [61], the Ludvigsen-Vickers energy-momenta (both based on the asymptotic and the point spinors) are also future directed and nonspacelike if $\mathcal{S}$ is the boundary of some compact spacelike hypersurface $\Sigma$ on which the dominant energy condition is satisfied and $\mathcal{S}$ is weakly future convex (or at least $\rho \leq 0$ ). Similarly, the Ludvigsen-Vickers definitions share the rigidity properties proven for the Dougan-Mason energy-momentum [354]: Under the same conditions the vanishing of the energy-momentum implies the flatness of the domain of dependence $D(\Sigma)$ of $\Sigma$.

In the weak field approximation [259] the difference $H_{\mathcal{S}_{r_{2}}}[\lambda, \bar{\lambda}]-H_{\mathcal{S}_{r_{1}}}[\lambda, \bar{\lambda}]$ is just the integral of $4 \pi G T_{a b} l^{a} \lambda^{B} \bar{\lambda}^{B^{\prime}}$ on the portion of $\mathcal{N}$ between the two 2-surfaces, where $T_{a b}$ is the linearized energy-momentum tensor: The increment of $H_{\mathcal{S}_{r}}[\lambda, \bar{\lambda}]$ on $\mathcal{N}$ is due only to the flux of the matter energy-momentum.

Since the Bondi-Sachs energy-momentum can be written as the integral of the Nester-Witten 2 -form on the cut in question at the null infinity with the asymptotic spinors, it is natural to expect that the first version of the Ludvigsen-Vickers energy-momentum tends to that of Bondi and Sachs. It was shown in $[259,340]$ that this expectation is, in fact, correct. The LudvigsenVickers mass was calculated for large spheres both for radiative and stationary spacetimes with $r^{-2}$ and $r^{-3}$ accuracy, respectively, in [338, 340].

Finally, on a small sphere of radius $r$ in non-vacuum the second definition gives [66] the expected result $(28)$, while in vacuum $[66,360]$ it is

$$
P_{\overline{\mathcal{S}}_{r}}^{\underline{A}} \underline{B}^{\prime}=\frac{1}{10 G} r^{5} T_{b c d}^{a} t^{b} t^{c} t^{d} \mathcal{E}_{A}^{A} \overline{\mathcal{E}}_{\bar{A}^{\prime}}{ }^{\prime}+\frac{4}{45 G} r^{6} t^{e}\left(\nabla_{e} T^{a}{ }_{b c d}\right) t^{b} t^{c} t^{d} \mathcal{E}_{\bar{A}}^{A} \overline{\mathcal{E}}_{\bar{A}^{\prime}}{ }^{\prime}+\mathcal{O}\left(r^{7}\right) .
$$

Thus its leading term is the energy-momentum of the matter fields and the Bel-Robinson momentum, respectively, seen by the observer $t^{a}$ at the vertex $p$. Thus, assuming that the matter 
fields satisfy the dominant energy condition, for small spheres this is an explicit proof that the Ludvigsen-Vickers quasi-local energy-momentum is future pointing and nonspacelike.

\subsection{The Dougan-Mason constructions}

\subsubsection{Holomorphic/anti-holomorphic spinor fields}

The original construction of Dougan and Mason [127] was introduced on the basis of sheaftheoretical arguments. Here we follow a slightly different, more 'pedestrian' approach, based mostly on [354, 356].

Following Dougan and Mason we define the spinor field $\lambda_{A}$ to be anti-holomorphic in case $m^{e} \nabla_{e} \lambda_{A}=m^{e} \Delta_{e} \lambda_{A}=0$, or holomorphic if $\bar{m}^{e} \nabla_{e} \lambda_{A}=\bar{m}^{e} \Delta_{e} \lambda_{A}=0$. Thus, this notion of holomorphicity/anti-holomorphicity is referring to the connection $\Delta_{e}$ on $\mathcal{S}$. While the notion of the holomorphicity/anti-holomorphicity of a function on $\mathcal{S}$ does not depend on whether the $\Delta_{e}$ or the $\delta_{e}$ operator is used, for tensor or spinor fields it does. Although the vectors $m^{a}$ and $\bar{m}^{a}$ are not uniquely determined (because their phase is not fixed), the notion of the holomorphicity/antiholomorphicity is well-defined, because the defining equations are homogeneous in $m^{a}$ and $\bar{m}^{a}$. Next suppose that there are at least two independent solutions of $\bar{m}^{e} \Delta_{e} \lambda_{A}=0$. If $\lambda_{A}$ and $\mu_{A}$ are any two such solutions, then $\bar{m}^{e} \Delta_{e}\left(\lambda_{A} \mu_{B} \varepsilon^{A B}\right)=0$, and hence by Liouville's theorem $\lambda_{A} \mu_{B} \varepsilon^{A B}$ is constant on $\mathcal{S}$. If this constant is not zero, then we call $\mathcal{S}$ generic, if it is zero then $\mathcal{S}$ will be called exceptional. Obviously, holomorphic $\lambda_{A}$ on a generic $\mathcal{S}$ cannot have any zero, and any two holomorphic spinor fields, e.g. $\lambda_{A}^{0}$ and $\lambda_{A}^{1}$, span the spin space at each point of $\mathcal{S}$ (and they can be chosen to form a normalized spinor dyad with respect to $\varepsilon_{A B}$ on the whole of $\mathcal{S}$ ). Expanding any holomorphic spinor field in this frame, the expanding coefficients turn out to be holomorphic functions, and hence constant. Therefore, on generic 2-surfaces there are precisely two independent holomorphic spinor fields. In the GHP formalism the condition of the holomorphicity of the spinor field $\lambda_{A}$ is that its components $\left(\lambda_{0}, \lambda_{1}\right)$ be in the kernel of $\mathcal{H}^{+}:=\Delta^{+} \oplus \mathcal{T}^{+}$. Thus for generic 2-surfaces ker $\mathcal{H}^{+}$with the constant $\varepsilon_{A B}$ would be a natural candidate for the spin space $\left(\mathbf{S}^{\underline{A}}, \varepsilon_{\underline{A}} \underline{B}\right)$ above. For exceptional 2-surfaces the kernel space ker $\mathcal{H}^{+}$ is either 2-dimensional but does not inherit a natural spin space structure, or it is higher than two dimensional. Similarly, the symplectic inner product of any two anti-holomorphic spinor fields is also constant, one can define generic and exceptional 2-surfaces as well, and on generic surfaces there are precisely two anti-holomorphic spinor fields. The condition of the anti-holomorphicity of $\lambda_{A}$ is $\lambda \in \operatorname{ker} \mathcal{H}^{-}:=\operatorname{ker}\left(\Delta^{-} \oplus \mathcal{T}^{-}\right)$. Then $\mathbf{S}^{A}=\operatorname{ker} \mathcal{H}^{-}$could also be a natural choice. Note that since the spinor fields whose holomorphicity/anti-holomorphicity is defined are unprimed, and these correspond to the anti-holomorphicity/holomorphicity, respectively, of the primed spinor fields of Dougan and Mason. Thus the main question is whether there exist generic 2-surfaces, and if they do, whether they are 'really generic', i.e. whether most of the physically important surfaces are generic or not.

\subsubsection{The genericity of the generic 2 -surfaces}

$\mathcal{H}^{ \pm}$are first order elliptic differential operators on certain vector bundles over the compact 2-surface $\mathcal{S}$, and their index can be calculated: index $\left(\mathcal{H}^{ \pm}\right)=2(1-g)$, where $g$ is the genus of $\mathcal{S}$. Therefore, for $\mathcal{S} \approx S^{2}$ there are at least two linearly independent holomorphic and at least two linearly independent anti-holomorphic spinor fields. The existence of the holomorphic/anti-holomorphic spinor fields on higher genus 2-surfaces is not guaranteed by the index theorem. Similarly, the index theorem does not guarantee that $\mathcal{S} \approx S^{2}$ is generic either: If the geometry of $\mathcal{S}$ is very special then the two holomorphic/anti-holomorphic spinor fields (which are independent as solutions of $\mathcal{H}^{ \pm} \lambda=0$ ) might be proportional to each other. For example, future marginally trapped surfaces (i.e. for which $\rho=0$ ) are exceptional from the point of view of holomorphic, and past

Living Reviews in Relativity

http: //www . livingreviews . org/lrr-2004-4 
marginally trapped surfaces $\left(\rho^{\prime}=0\right)$ from the point of view of anti-holomorphic spinors. Furthermore, there are surfaces with at least three linearly independent holomorphic/anti-holomorphic spinor fields. However, small generic perturbations of the geometry of an exceptional 2-surface $\mathcal{S}$ with $S^{2}$ topology make $\mathcal{S}$ generic.

Finally, we note that several first order differential operators can be constructed from the chiral irreducible parts $\Delta^{ \pm}$and $\mathcal{T}^{ \pm}$of $\Delta_{e}$, given explicitly by Equation (25). However, only four of them, the Dirac-Witten operator $\Delta:=\Delta^{+} \oplus \Delta^{-}$, the twistor operator $\mathcal{T}:=\mathcal{T}^{+} \oplus \mathcal{T}^{-}$, and the holomorphy and anti-holomorphy operators $\mathcal{H}^{ \pm}$, are elliptic (which ellipticity, together with the compactness of $\mathcal{S}$, would guarantee the finiteness of the dimension of their kernel), and it is only $\mathcal{H}^{ \pm}$that have 2-complex-dimensional kernel in the generic case. This purely mathematical result gives some justification for the choices of Dougan and Mason: The spinor fields $\lambda_{A}^{A}$ that should be used in the Nester-Witten 2-form are either holomorphic or anti-holomorphic. The construction does not work for exceptional 2-surfaces.

\subsubsection{Positivity properties}

One of the most important properties of the Dougan-Mason energy-momenta is that they are future pointing nonspacelike vectors, i.e. the corresponding masses and energies are non-negative. Explicitly [127], if $\mathcal{S}$ is the boundary of some compact spacelike hypersurface $\Sigma$ on which the dominant energy condition holds, furthermore if $\mathcal{S}$ is weakly future convex (in fact, $\rho \leq 0$ is enough), then the holomorphic Dougan-Mason energy-momentum is a future pointing non-spacelike vector, and, analogously, the anti-holomorphic energy-momentum is future pointing and non-spacelike if $\rho^{\prime} \geq 0$. As Bergqvist [61] stressed (and we noted in Section 8.1.3), Dougan and Mason used only the $\Delta^{+} \lambda=0$ (and in the anti-holomorphic construction the $\Delta^{-} \lambda=0$ ) half of the 'propagation law' in their positivity proof. The other half is needed only to ensure the existence of two spinor fields. Thus that might be Equation (59) of the Ludvigsen-Vickers construction, or $\mathcal{T}^{+} \lambda=0$ in the holomorphic Dougan-Mason construction, or even $\mathcal{T}^{+} \lambda=k \sigma^{\prime} \psi_{2}^{\prime} \lambda_{0}$ for some constant $k$, a 'deformation' of the holomorphicity considered by Bergqvist [61]. In fact, the propagation law may even be $\bar{m}^{a} \Delta_{a} \lambda_{B}=\tilde{f}_{B}{ }^{C} \lambda_{C}$ for any spinor field $\tilde{f}_{B}^{C}$ satisfying $\pi^{-B}{ }_{A} \tilde{f}_{B}^{C}=\tilde{f}_{A}^{B} \pi^{+C}{ }_{B}=0$. This ensures the positivity of the energy under the same conditions and that $\varepsilon^{A B} \lambda_{A} \mu_{B}$ is still constant on $\mathcal{S}$ for any two solutions $\lambda_{A}$ and $\mu_{A}$, making it possible to define the norm of the resulting energy-momentum, i.e. the mass.

In the asymptotically flat spacetimes the positive energy theorems have a rigidity part too, namely the vanishing of the energy-momentum (and, in fact, even the vanishing of the mass) implies flatness. There are analogous theorems for the Dougan-Mason energy-momenta too [354, 356]. Namely, under the conditions of the positivity proof

1. $P_{\mathcal{S}} \underline{B}^{\prime}$ is zero iff $D(\Sigma)$ is flat, which is also equivalent to the vanishing of the quasi-local energy, $E_{\mathcal{S}}:=\frac{1}{\sqrt{2}}\left(P_{\mathcal{S}}^{00^{\prime}}+P_{\mathcal{S}}^{11^{\prime}}\right)=0$, and

2. $P \mathcal{A}_{\mathcal{S}} \underline{B}^{\prime}$ is null (i.e. the quasi-local mass is zero) iff $D(\Sigma)$ is a $p p$-wave geometry and the matter is pure radiation.

In particular [365], for a coupled Einstein-Yang-Mills system (with compact, semisimple gauge groups) the zero quasi-local mass configurations are precisely the $p p$-wave solutions found by Güven [167]. Therefore, in contrast to the asymptotically flat cases, the vanishing of the mass does not imply the flatness of $D(\Sigma)$. Since, as we will see below, the Dougan-Mason masses tend to the ADM mass at spatial infinity, seemingly there is a contradiction between the rigidity part of the positive mass theorems and the Result 2 above. However, this contradiction is only apparent. In fact, according to one of the possible positive mass proofs [24], the vanishing of the ADM mass implies the existence of a constant null vector field on $D(\Sigma)$, and then the flatness follows from the 
incompatibility of the conditions of the asymptotic flatness and the existence of a constant null vector field: The only asymptotically flat spacetime admitting a constant null vector field is the flat spacetime.

These results show some sort of rigidity of the matter + gravity system (where the latter satisfies the dominant energy condition) even at the quasi-local level, which is much more manifest from the following equivalent form of Results 1 and 2: Under the same conditions $D(\Sigma)$ is flat if and only if there exist two linearly independent spinor fields on $\mathcal{S}$ which are constant with respect to $\Delta_{e}$, and $D(\Sigma)$ is a $p p$-wave geometry and the matter is pure radiation if and only if there exists a $\Delta_{e^{-}}$ constant spinor field on $\mathcal{S}$ [356]. Thus the full information that $D(\Sigma)$ is flat/pp-wave is completely encoded not only in the usual initial data on $\Sigma$, but in the geometry of the boundary of $\Sigma$, too. In Section 13.5 we return to the discussion of this phenomenon, where we will see that, assuming that $\mathcal{S}$ is future and past convex, the whole line element of $D(\Sigma)$ (and not only the information that it is some $p p$-wave geometry) is determined by the 2 -surface data on $\mathcal{S}$.

Comparing Results 1 and 2 above with the properties of the quasi-local energy-momentum (and angular momentum) listed in Section 2.2.3, the similarity is obvious: $P_{\mathcal{S}}^{\underline{A}} \underline{B}^{\prime}=0$ characterizes the 'quasi-local vacuum state' of general relativity, while $m_{\mathcal{S}}=0$ is equivalent to 'pure radiative quasilocal states'. The equivalence of $E_{\mathcal{S}}=0$ and the flatness of $D(\Sigma)$ shows that curvature always yields positive energy, or, in other words, with this notion of energy no classical symmetry breaking can occur in general relativity: The 'quasi-local ground states' (defined by $E_{\mathcal{S}}=0$ ) are just the 'quasi-local vacuum states' (defined by the trivial value of the field variables on $D(\Sigma)$ ) [354], in contrast, for example, to the well known $\phi^{4}$ theories.

\subsubsection{The various limits}

Both definitions give the same standard expression for round spheres [126]. Although the limit of the Dougan-Mason masses for round spheres in Reissner-Nordström spacetime gives the correct irreducible mass of the Reissner-Nordström black hole on the horizon, the constructions do not work on the surface of bifurcation itself, because that is an exceptional 2-surface. Unfortunately, without additional restrictions (e.g. the spherical symmetry of the 2-surfaces in a spherically symmetric spacetime) the mass of the exceptional 2-surfaces cannot be defined in a limiting process, because, in general, the limit depends on the family of generic 2-surfaces approaching the exceptional one [356].

Both definitions give the same, expected results in the weak field approximation and for large spheres at spatial infinity: Both tend to the ADM energy-momentum [127]. In non-vacuum both definitions give the same, expected expression (28) for small spheres, in vacuum they coincide in the $r^{5}$ order with that of Ludvigsen and Vickers, but in the $r^{6}$ order they differ from each other: The holomorphic definition gives Equation (61), but in the analogous expression for the antiholomorphic energy-momentum the numerical coefficient $4 /(45 G)$ is replaced by $1 /(9 G)[126]$. The Dougan-Mason energy-momenta have also been calculated for large spheres of constant Bondi-type radial coordinate value $r$ near future null infinity [126]. While the anti-holomorphic construction tends to the Bondi-Sachs energy-momentum, the holomorphic one diverges in general. In stationary spacetimes they coincide and both give the Bondi-Sachs energy-momentum. At the past null infinity it is the holomorphic construction which reproduces the Bondi-Sachs energy-momentum and the anti-holomorphic diverges.

We close this section with some caution and general comments on a potential gauge ambiguity in the calculation of the various limits. By the definition of the holomorphic and anti-holomorphic spinor fields they are associated with the 2-surface $\mathcal{S}$ only. Thus if $\mathcal{S}^{\prime}$ is another 2 -surface, then there is no natural isomorphism between the space - for example of the anti-holomorphic spinor fields ker $\mathcal{H}^{-}(\mathcal{S})$ on $\mathcal{S}$ - and ker $\mathcal{H}^{-}\left(\mathcal{S}^{\prime}\right)$ on $\mathcal{S}^{\prime}$, even if both surfaces are generic and hence there are

Living Reviews in Relativity

http: //www. livingreviews . org//rr-2004-4 
isomorphisms between them ${ }^{12}$. This (apparently 'only theoretical') fact has serious pragmatic consequences. In particular, in the small or large sphere calculations we compare the energy-momenta, and hence the holomorphic or anti-holomorphic spinor fields also, on different surfaces. For example [360], in the small sphere approximation every spin coefficient and spinor component in the GHP dyad and metric component in some fixed coordinate system $(\zeta, \bar{\zeta})$ is expanded as a series of $r$, like $\lambda_{\mathbf{A}}(r, \zeta, \bar{\zeta})=\lambda_{\mathbf{A}}{ }^{(0)}(\zeta, \bar{\zeta})+r \lambda_{\mathbf{A}}{ }^{(1)}(\zeta, \bar{\zeta})+\cdots+r^{k} \lambda_{\mathbf{A}}{ }^{(k)}(\zeta, \bar{\zeta})+\mathcal{O}\left(r^{k+1}\right)$. Substituting all such expansions and the asymptotic solutions of the Bianchi identities for the spin coefficients and metric functions into the differential equations defining the holomorphic/anti-holomorphic spinors, we obtain a hierarchical system of differential equations for the expansion coefficients $\lambda_{\mathbf{A}}{ }^{(0)}, \lambda_{\mathbf{A}}{ }^{(1)}$, $\ldots$, etc. It turns out that the solutions of this system of equations with accuracy $r^{k}$ form a $2 k$ rather than the expected two complex dimensional space. $2(k-1)$ of these $2 k$ solutions are 'gauge' solutions, and they correspond in the approximation with given accuracy to the unspecified isomorphism between the space of the holomorphic/anti-holomorphic spinor fields on surfaces of different radii. Obviously, similar 'gauge' solutions appear in the large sphere expansions, too. Therefore, without additional gauge fixing, in the expansion of a quasi-local quantity only the leading nontrivial term will be gauge-independent. In particular, the $r^{6}$ order correction in Equation (61) for the Dougan-Mason energy-momenta is well-defined only as a consequence of a natural gauge choice $^{13}$. Similarly, the higher order corrections in the large sphere limit of the anti-holomorphic Dougan-Mason energy-momentum are also ambiguous unless a 'natural' gauge choice is made. Such a choice is possible in stationary spacetimes.

\subsection{A specific construction for the Kerr spacetime}

Logically, this specific construction perhaps would have to be presented only in Section 12, but the technique that it is based on may justify its placing here.

By investigating the propagation law $(59,60)$ of Ludvigsen and Vickers, for the Kerr spacetimes Bergqvist and Ludvigsen constructed a natural flat, (but non-symmetric) metric connection [67]. Writing the new covariant derivative in the form $\tilde{\nabla}_{A A^{\prime}} \lambda_{B}=\nabla_{A A^{\prime}} \lambda_{B}+\Gamma_{A A^{\prime} B^{C}} \lambda_{C}$, the 'correction' term $\Gamma_{A A^{\prime} B}{ }^{C}$ could be given explicitly in terms of the GHP spinor dyad (adapted to the two principal null directions), the spin coefficients $\rho, \tau$ and $\tau^{\prime}$, and the curvature component $\psi_{2} . \Gamma_{A A^{\prime} B^{C}}$ admits a potential [68]: $\Gamma_{A A^{\prime} B C}=-\nabla_{(C} B^{B^{\prime}} H_{B) A A^{\prime} B^{\prime}}$, where $H_{A B A^{\prime} B^{\prime}}:=\frac{1}{2} \rho^{-3}(\rho+\bar{\rho}) \psi_{2} O_{A} O_{B} \bar{o}_{A^{\prime}} \bar{o}_{B^{\prime}}$. However, this potential has the structure $H_{a b}=f l_{a} l_{b}$ appearing in the form of the metric $g_{a b}=g_{a b}^{0}+f l_{a} l_{b}$ for the Kerr-Schild spacetimes, where $g_{a b}^{0}$ is the flat metric. In fact, the flat connection $\tilde{\nabla}_{e}$ above could be introduced for general Kerr-Schild metrics [170], and the corresponding 'correction term' $\Gamma_{A A^{\prime} B C}$ could be used to find easily the Lánczos potential for the Weyl curvature [10].

Since the connection $\tilde{\nabla}_{A A^{\prime}}$ is flat and annihilates the spinor metric $\varepsilon_{A B}$, there are precisely two linearly independent spinor fields, say $\lambda_{A}^{0}$ and $\lambda_{A}^{1}$, that are constant with respect to $\tilde{\nabla}_{A A^{\prime}}$ and form a normalized spinor dyad. These spinor fields are asymptotically constant. Thus it is natural to choose the spin space $\left(\mathbf{S}^{\underline{A}}, \varepsilon_{\underline{A}} \underline{B}\right)$ to be the space of the $\tilde{\nabla}_{a}$-constant spinor fields, independently of the 2 -surface $\mathcal{S}$.

A remarkable property of these spinor fields is that the Nester-Witten 2-form built from them is closed: $d u\left(\lambda \underline{A}, \bar{\lambda}^{\prime} \underline{\prime}^{\prime}\right)=0$. This implies that the quasi-local energy-momentum depends only on the homology class of $\mathcal{S}$, i.e. if $\mathcal{S}_{1}$ and $\mathcal{S}_{2}$ are 2-surfaces such that they form the boundary of some hypersurface in $M$, then $P_{\mathcal{S}_{1}}^{\underline{A}}{ }^{\prime}=P_{\mathcal{S}_{2}} \underline{B}^{\prime}$, and if $\mathcal{S}$ is the boundary of some hypersurface, then

\footnotetext{
${ }^{12}$ Recall that, similarly, we did not have any natural isomorphism between the 2-surface twistor spaces, discussed in Section 7.2.1, on different 2-surfaces.

${ }^{13}$ Clearly, for the Ludvigsen-Vickers energy-momentum no such ambiguity is present, because the part (59) of their propagation law defines a natural isomorphism between the space of the Ludvigsen-Vickers spinors on the different 2-surfaces.
} 
$P_{\mathcal{S}}^{A} \underline{B}^{\prime}=0$. In particular, for two-spheres that can be shrunk to a point the energy-momentum is zero, but for those that can be deformed to a cut of the future null infinity the energy-momentum is that of Bondi and Sachs.

Living Reviews in Relativity

http://www. livingreviews . org/lrr-2004-4 


\section{Quasi-Local Spin-Angular Momentum}

In this section we review three specific quasi-local spin-angular momentum constructions that are (more or less) 'quasi-localizations' of Bramson's expression at null infinity. Thus the quasilocal spin-angular momentum for the closed, orientable spacelike 2-surface $\mathcal{S}$ will be sought in the form (17). Before considering the specific constructions themselves we summarize the most important properties of the general expression of Equation (17). Since the most detailed discussion of Equation (17) is given probably in [360, 363], the subsequent discussions will be based on them.

First, observe that the integral depends on the spinor dyad algebraically, thus it is enough to specify the dyad only at the points of $\mathcal{S}$. Obviously, $J_{\mathcal{S}} \underline{B}$ transforms like a symmetric second rank spinor under constant $S L(2, \mathbf{C})$ transformations of the dyad $\left\{\lambda_{A}^{A}\right\}$. Second, suppose that the spacetime is flat, and let $\left\{\lambda \frac{A}{A}\right\}$ be constant. Then the corresponding 1-form basis $\left\{\vartheta \frac{a}{a}\right\}$ is the constant Cartesian one, which consists of exact 1-forms. Then since the Bramson superpotential $w\left(\lambda^{\underline{A}}, \lambda^{\underline{B}}\right)_{a b}$ is the anti-self-dual part (in the name indices) of $\vartheta^{a} \frac{a}{b} \frac{b}{b}-\vartheta \frac{a}{b} \vartheta^{\frac{b}{a}}$, which is also exact, for such spinor bases Equation (17) gives zero. Therefore, the integral of Bramson's superpotential (17) measures the non-integrability of the 1-form basis $\vartheta \frac{A}{a} \underline{A}^{\prime}=\lambda \frac{A}{A} \bar{\lambda}^{A^{\prime}}{ }^{\prime}$, i.e. $J \frac{A}{\mathcal{S}} \underline{B}$ is a measure of how much the actual 1-form basis is 'distorted' by the curvature relative to the constant basis of Minkowski spacetime.

Thus the only question is how to specify a spin frame on $\mathcal{S}$ to be able to interpret $J \frac{A}{\mathcal{S}} \underline{B}$ as angular momentum. It seems natural to choose those spinor fields that were used in the definition of the quasi-local energy-momenta in the previous Section 8. At first sight this may appear to be only an ad hoc idea, but, recalling that in Section 8 we interpreted the elements of the spin spaces $\left(\mathbf{S}^{A}, \varepsilon_{\underline{A}} \underline{B}\right)$ as the 'spinor constituents of the quasi-translations of $\mathcal{S}$ ', we can justify such a choice. Based on our experience with the superpotentials for the various conserved quantities, the quasi-local angular momentum can be expected to be the integral of something like 'superpotential' $\times$ 'quasi-rotation generator', and the 'superpotential' is some expression in the first derivative of the basic variables, actually the tetrad or spinor basis. Since, however, Bramson's superpotential is an algebraic expression of the basic variables, and the number of the derivatives in the expression for the angular momentum should be one, the angular momentum expressions based on Bramson's superpotential must contain the derivative of the 'quasi-rotations', i.e. (possibly a combination of) the 'quasi-translations'. Since, however, such an expression cannot be sensitive to the 'change of the origin', they can be expected to yield only the spin part of the angular momentum.

The following two specific constructions differ from each other only in the choice for the spin space $\left(\mathbf{S}^{A}, \varepsilon_{\underline{A}} \underline{B}\right)$, and correspond to the energy-momentum constructions of the previous Section 8 . The third construction (valid only in the Kerr spacetimes) is based on the sum of two terms, where one is Bramson's expression, and uses the spinor fields of Section 8.3. Thus the present section is not independent of Section 8, and for the discussion of the choice of the spin spaces $\left(\mathbf{S}^{A}, \varepsilon_{\underline{A}} \underline{B}\right)$ we refer to that.

Another suggestion for the quasi-local spatial angular momentum, proposed by Liu and Yau [253], will be introduced in Section 10.4.1.

\subsection{The Ludvigsen-Vickers angular momentum}

Under the conditions that ensured the Ludvigsen-Vickers construction for the energy-momentum would work in Section 8.1, the definition of their angular momentum is straightforward [259]. Since in Minkowski spacetime the Ludvigsen-Vickers spinors are just the restriction to $\mathcal{S}$ of the constant spinor fields, by the general remark above the Ludvigsen-Vickers spin-angular momentum is zero in Minkowski spacetime.

Using the asymptotic solution of the Einstein-Maxwell equations in a Bondi-type coordinate system it has been shown in [259] that the Ludvigsen-Vickers spin-angular momentum tends to 
that of Bramson at future null infinity. For small spheres [360] in non-vacuum it reproduces precisely the expected result (29), and in vacuum it is

$$
J_{\mathcal{S}_{r}}^{\underline{A}} \underline{\underline{B}}=\frac{4}{45 G} r^{5} T_{A A^{\prime} B B^{\prime} C C^{\prime} D D^{\prime}} t^{A A^{\prime}} t^{B B^{\prime}} t^{C C^{\prime}}\left(r t^{D^{\prime} E} \varepsilon^{D F} \mathcal{E}_{(E}^{\underline{A}} \mathcal{E}_{F)}^{\underline{B}}\right)+\mathcal{O}\left(r^{7}\right)
$$

We stress that in both the vacuum and non-vacuum cases the factor $r t^{D^{\prime} E} \varepsilon^{D F} \mathcal{E}_{(E}^{A} \mathcal{E}_{F)}^{B}$, interpreted in Section 4.2.2 as an average of the boost-rotation Killing fields that vanish at $p$, emerges naturally. No (approximate) boost-rotation Killing field was put into the general formulae by hand.

\subsection{Holomorphic/anti-holomorphic spin-angular momenta}

Obviously, the spin-angular momentum expressions based on the holomorphic and anti-holomorphic spinor fields [358] on generic 2-surfaces are genuinely quasi-local. Since in Minkowski spacetime the restriction of the two constant spinor fields to any 2-surface are constant, and hence holomorphic and anti-holomorphic at the same time, both the holomorphic and anti-holomorphic spin-angular momenta are vanishing. Similarly, for round spheres both definitions give zero [363], as it could be expected in a spherically symmetric system. The anti-holomorphic spin-angular momentum has already been calculated for axi-symmetric 2-surfaces $\mathcal{S}$ for which the anti-holomorphic DouganMason energy-momentum is null, i.e. for which the corresponding quasi-local mass is zero. (As we saw in Section 8.2.3, this corresponds to a $p p$-wave geometry and pure radiative matter fields on $D(\Sigma)[354,356]$.) This null energy-momentum vector turned out to be an eigenvector of the anti-symmetric spin-angular momentum tensor $J_{\mathcal{S}}^{\underline{a}} \underline{\underline{b}}$, which, together with the vanishing of the quasi-local mass, is equivalent to the proportionality of the (null) energy-momentum vector and the Pauli-Lubanski spin [358], where the latter is defined by

$$
S_{\mathcal{S}}^{\underline{a}}:=\frac{1}{2} \varepsilon^{\underline{a}} \underline{\underline{b}} \underline{c} \underline{d} P_{\mathcal{S}}^{\underline{b}} J_{\mathcal{S}}^{\underline{c}} \underline{d} .
$$

This is a known property of the zero-rest-mass fields in Poincaré invariant quantum field theories [168].

Both the holomorphic and anti-holomorphic spin-angular momenta were calculated for small spheres [360]. In non-vacuum the holomorphic spin-angular momentum reproduces the expected result (29), and, apart from a minus sign, the anti-holomorphic construction does also. In vacuum both definitions give exactly Equation (62).

In general the anti-holomorphic and the holomorphic spin-angular momenta are diverging near the future null infinity of Einstein-Maxwell spacetimes as $r$ and $r^{2}$, respectively. However, the coefficient of the diverging term in the anti-holomorphic expression is just the spatial part of the Bondi-Sachs energy-momentum. Thus the anti-holomorphic spin-angular momentum is finite in the centre-of-mass frame, and hence it seems to describe only the spin part of the gravitational field. In fact, the Pauli-Lubanski spin (63) built from this spin-angular momentum and the anti-holomorphic Dougan-Mason energy-momentum is always finite, free of 'gauge' ambiguities discussed in Section 8.2.4, and is built only from the gravitational data even in the presence of electromagnetic fields. In stationary spacetimes both constructions are finite and coincide with the 'standard' expression (34). Thus the anti-holomorphic spin-angular momentum defines an intrinsic angular momentum at the future null infinity. Note that this angular momentum is free of supertranslation ambiguities, because it is defined on the given cut in terms of the solutions of elliptic differential equations. These solutions can be interpreted as the spinor constituents of certain boost-rotation BMS vector fields, but the definition of this angular momentum is not based on them [363].

Living Reviews in Relativity

http://www. livingreviews.org/lrr-2004-4 


\subsection{A specific construction for the Kerr spacetime}

The angular momentum of Bergqvist and Ludvigsen [68] for the Kerr spacetime is based on their special flat, non-symmetric but metric connection explained briefly in Section 8.3, but their idea is not simply the use of the two $\tilde{\nabla}_{e}$-constant spinor fields in Bramson's superpotential. Rather, in the background of their approach there are twistor-theoretical ideas. (The twistor-theoretic aspects of the analogous flat connection for the general Kerr-Schild class are discussed in [170].)

The main idea is that while the energy-momentum is a single four-vector in the dual of the Hermitian subspace of $\mathbf{S}^{\underline{A}} \otimes \overline{\mathbf{S}}^{\underline{B}^{\prime}}$, the angular momentum is not only an anti-symmetric tensor over the same space, but should depend on the 'origin', a point in a 4-dimensional affine space $M_{0}$ as well, and should transform in a specific way under the translation of the 'origin'. Bergqvist and Ludvigsen defined the affine space $M_{0}$ to be the space of the solutions $X_{a}$ of $\tilde{\nabla}_{a} X_{b}=g_{a b}-H_{a b}$, and showed that $M_{0}$ is, in fact, a real, four dimensional affine space. Then, for a given $X_{A A^{\prime}}$, to each $\tilde{\nabla}_{a}$-constant spinor field $\lambda^{A}$ they associate a primed spinor field by $\mu_{A^{\prime}}:=X_{A^{\prime} A^{\prime}} \lambda^{A}$. This $\mu_{A^{\prime}}$ turns out to satisfy the modified valence 1 twistor equation $\tilde{\nabla}_{A\left(A^{\prime}\right.} \mu_{\left.B^{\prime}\right)}=-H_{A A^{\prime} B B^{\prime}} \lambda^{B}$. Finally, they form the 2-form

$$
\left.W\left(X, \lambda^{\underline{A}}, \lambda^{\underline{B}}\right)_{a b}:=\mathrm{i}\left[\lambda \frac{A}{A} \nabla_{B B^{\prime}}\left(X_{A^{\prime} C} \varepsilon^{C D} \lambda \frac{B}{D}\right)-\lambda_{B}^{A} \nabla_{A A^{\prime}}\left(X_{B^{\prime} C} \varepsilon^{C D} \lambda \frac{B}{D}\right)+\varepsilon_{A^{\prime} B^{\prime}} \lambda \frac{A}{(A} \lambda \frac{\underline{B}}{B}\right)\right],
$$

and define the angular momentum $J_{\mathcal{S}} \underline{\underline{B}}(X)$ with respect to the origin $X_{a}$ as $1 /(8 \pi G)$ times the integral of $W(X, \lambda \underline{A}, \lambda \underline{B})_{a b}$ on some closed, orientable spacelike 2-surface $\mathcal{S}$. Since this $W_{a b}$ is closed, $\nabla_{[a} W_{b c]}=0$ (similarly to the Nester-Witten 2-form in Section 8.3), the integral $J_{\mathcal{S}} \underline{\underline{B}}(X)$ depends only on the homology class of $\mathcal{S}$. Under the 'translation' $X_{e} \mapsto X_{e}+a_{e}$ of the 'origin' by a

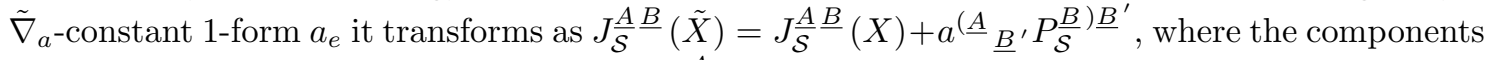
$a_{\underline{A}} \underline{B}^{\prime}$ are taken with respect to the basis $\left\{\lambda \frac{A}{A}\right\}$ in the solution space. Unfortunately, no explicit expression for the angular momentum in terms of the Kerr parameters $m$ and $a$ is given. 


\section{The Hamilton-Jacobi Method}

If one is concentrating only on the introduction and study of the properties of the quasi-local quantities, but not interested in the detailed structure of the quasi-local (Hamiltonian) phase space, then perhaps the most natural way to derive the general formulae is to follow the Hamilton-Jacobi method. This was done by Brown and York in deriving their quasi-local energy expression [96, 97]. However, the Hamilton-Jacobi method in itself does not yield any specific construction. Rather, the resulting general expression is similar to a superpotential in the Lagrangian approaches, which should be completed by a choice for the reference configuration and for the generator vector field of the physical quantity (see Section 3.3.3). In fact, the 'Brown-York quasi-local energy' is not a single expression with a single well-defined prescription for the reference configuration. The same general formula with several other, mathematically inequivalent definitions for the reference configurations are still called the 'Brown-York energy'. A slightly different general expression was used by Kijowski [237], Epp [133], and Liu and Yau [253]. Although the former follows a different route to derive his expression and the latter two are not connected directly to the canonical analysis (and, in particular, to the Hamilton-Jacobi method), the formalism and techniques that are used justify their presentation in this section.

The present section is based mostly on the original papers [96, 97] by Brown and York. Since, however, this is the most popular approach to finding quasi-local quantities and is the subject of very active investigations, especially from the point of view of the applications in black hole physics, this section is perhaps less complete than the previous ones. The expressions of Kijowski, Epp, and Liu and Yau will be treated in the formalism of Brown and York.

\subsection{The Brown-York expression}

\subsubsection{The main idea}

To motivate the main idea behind the Brown-York definition [96, 97], let us consider first a classical mechanical system of $n$ degrees of freedom with configuration manifold $Q$ and Lagrangian $L: T Q \times \mathbb{R} \rightarrow \mathbb{R}$ (i.e. the Lagrangian is assumed to be first order and may depend on time explicitly) For given initial and final configurations, $\left(q_{1}^{a}, t_{1}\right)$ and $\left(q_{2}^{a}, t_{2}\right)$, respectively, the corresponding action functional is $I^{1}[q(t)]:=\int_{t_{1}}^{t_{2}} L\left(q^{a}(t), \dot{q}^{a}(t), t\right) d t$, where $q^{a}(t)$ is a smooth curve in $Q$ from $q^{a}\left(t_{1}\right)=q_{1}^{a}$ to $q^{a}\left(t_{2}\right)=q_{2}^{a}$ with tangent $\dot{q}^{a}(t)$ at $t$. (The pair $\left(q^{a}(t), t\right)$ may be called a history or world line in the 'spacetime' $Q \times \mathbb{R}$.) Let $\left(q^{a}(u, t(u)), t(u)\right)$ be a smooth 1-parameter deformation of this history, i.e. for which $\left(q^{a}(0, t(0)), t(0)\right)=\left(q^{a}(t), t\right)$, and $u \in(-\epsilon, \epsilon)$ for some $\epsilon>0$. Then, denoting the derivative with respect to the deformation parameter $u$ at $u=0$ by $\delta$, one has the well known expression

$$
\delta I^{1}[q(t)]=\int_{t_{1}}^{t_{2}}\left(\frac{\partial L}{\partial q^{a}}-\frac{d}{d t} \frac{\partial L}{\partial \dot{q}^{a}}\right)\left(\delta q^{a}-\dot{q}^{a} \delta t\right) d t+\left.\frac{\partial L}{\partial \dot{q}^{a}} \delta q^{a}\right|_{t_{1}} ^{t_{2}}-\left.\left(\frac{\partial L}{\partial \dot{q}^{a}} \dot{q}^{a}-L\right) \delta t\right|_{t_{1}} ^{t_{2}}
$$

Therefore, introducing the Hamilton-Jacobi principal function $S^{1}\left(q_{1}^{a}, t_{1} ; q_{2}^{a}, t_{2}\right)$ as the value of the action on the solution $q^{a}(t)$ of the equations of motion from $\left(q_{1}^{a}, t_{1}\right)$ to $\left(q_{2}^{a}, t_{2}\right)$, the derivative of $S^{1}$ with respect to $q_{2}^{a}$ gives the canonical momenta $p_{a}^{1}:=\left(\partial L / \partial \dot{q}^{a}\right)$, while its derivative with respect to $t_{2}$ gives minus the energy, $-E^{1}=-\left(p_{a}^{1} \dot{q}^{a}-L\right)$, at $t_{2}$. Obviously, neither the action $I^{1}$ nor the principal function $S^{1}$ are unique: $I[q(t)]:=I^{1}[q(t)]-I^{0}[q(t)]$ for any $I^{0}[q(t)]$ of the form $\int_{t_{1}}^{t_{2}}(d h / d t) d t$ with arbitrary smooth function $h=h\left(q^{a}(t), t\right)$ is an equally good action for the same dynamics. Clearly, the subtraction term $I^{0}[q(t)]$ alters both the canonical momenta and the energy according to $p_{a}^{1} \mapsto p_{a}=p_{a}^{1}-\left(\partial h / \partial q^{a}\right)$ and $E^{1} \mapsto E=E^{1}+(\partial h / \partial t)$, respectively.

Living Reviews in Relativity

http: //www . livingreviews . org/lrr-2004-4 


\subsubsection{The variation of the action and the surface stress-energy tensor}

The main idea of Brown and York [96, 97] is to calculate the analogous variation of an appropriate first order action of general relativity (or of the coupled matter + gravity system) and isolate the boundary term that could be analogous to the energy $E$ above. To formulate this idea mathematically, they considered a compact spacetime domain $D$ with topology $\Sigma \times\left[t_{1}, t_{2}\right]$ such that $\Sigma \times\{t\}$ correspond to compact spacelike hypersurfaces $\Sigma_{t}$; these form a smooth foliation of $D$ and the 2-surfaces $\mathcal{S}_{t}:=\partial \Sigma_{t}$ (corresponding to $\partial \Sigma \times\{t\}$ ) form a foliation of the timelike 3-boundary ${ }^{3} B$ of $D$. Note that this $D$ is not a globally hyperbolic domain ${ }^{14}$. To ensure the compatibility of the dynamics with this boundary, the shift vector is usually chosen to be tangent to $\mathcal{S}_{t}$ on ${ }^{3} B$. The orientation of ${ }^{3} B$ is chosen to be outward pointing, while the normals both of $\Sigma_{1}:=\Sigma_{t_{1}}$ and $\Sigma_{2}:=\Sigma_{t_{2}}$ to be future pointing. The metric and extrinsic curvature on $\Sigma_{t}$ will be denoted, respectively, by $h_{a b}$ and $\chi_{a b}$, those on ${ }^{3} B$ by $\gamma_{a b}$ and $\Theta_{a b}$.

The primary requirement of Brown and York on the action is to provide a well-defined variational principle for the Einstein theory. This claim leads them to choose for $I^{1}$ the 'trace $K$ action' (or, in the present notation, rather the 'trace $\chi$ action') for general relativity [405, 406, 387], and the action for the matter fields may be included. (For the minimal, non-derivative couplings the presence of the matter fields does not alter the subsequent expressions.) However, as Geoff Hayward pointed out [178], to have a well-defined variational principle, the 'trace $\chi$ action' should in fact be completed by two 2-surface integrals, one on $\mathcal{S}_{1}$ and the other on $\mathcal{S}_{2}$. Otherwise, as a consequence of the edges $\mathcal{S}_{1}$ and $\mathcal{S}_{2}$, called the 'joints' (i.e. the non-smooth parts of the boundary $\partial D$ ), the variation of the metric at the points of the edges $\mathcal{S}_{1}$ and $\mathcal{S}_{2}$ could not be arbitrary. (See also $[177,237,77,95]$, where the 'orthogonal boundaries assumption' is also relaxed.) Let $\eta_{1}$ and $\eta_{2}$ be the scalar product of the outward pointing normal of ${ }^{3} B$ and the future pointing normal of $\Sigma_{1}$ and of $\Sigma_{2}$, respectively. Then, varying the spacetime metric, for the variation of the corresponding principal function $S^{1}$ they obtained

$$
\begin{aligned}
\delta S^{1}= & \int_{\Sigma_{2}} \frac{1}{16 \pi G} \sqrt{|h|}\left(\chi^{a b}-\chi h^{a b}\right) \delta h_{a b} d^{3} x- \\
& -\int_{\Sigma_{1}} \frac{1}{16 \pi G} \sqrt{|h|}\left(\chi^{a b}-\chi h^{a b}\right) \delta h_{a b} d^{3} x- \\
& -\int_{{ }^{B} B} \frac{1}{16 \pi G} \sqrt{|\gamma|}\left(\Theta^{a b}-\Theta \gamma^{a b}\right) \delta \gamma_{a b} d^{3} x- \\
& -\frac{1}{8 \pi G} \oint_{\mathcal{S}_{2}} \tanh ^{-1} \eta_{2} \delta \sqrt{|q|} d^{2} x+\frac{1}{8 \pi G} \oint_{\mathcal{S}_{1}} \tanh ^{-1} \eta_{1} \delta \sqrt{|q|} d^{2} x .
\end{aligned}
$$

The first two terms together correspond to the term $\left.p_{a}^{1} \delta q^{a}\right|_{t_{1}} ^{t_{2}}$ of Equation (65), and, in fact, the familiar ADM expression for the canonical momentum $\tilde{p}^{a b}$ is just $\frac{1}{16 \pi G} \sqrt{|h|}\left(\chi^{a b}-\chi h^{a b}\right)$. The last two terms give the effect of the presence of the non-differentiable 'joints'. Therefore, it is the third term that should be analogous to the third term of Equation (65). In fact, roughly, this is proportional to the proper time separation of the 'instants' $\Sigma_{1}$ and $\Sigma_{2}$, and it is reasonable to identify its coefficient as some (quasi-local) analog of the energy. However, just as in the case of the mechanical system, the action (and the corresponding principal function) is not unique, and the principal function should be written as $S:=S^{1}-S^{0}$, where $S^{0}$ is assumed to be an arbitrary function of the 3-metric on the boundary $\partial D=\Sigma_{2} \cup{ }^{3} B \cup \Sigma_{1}$. Then

$$
\tau^{a b}:=-\frac{2}{\sqrt{|\gamma|}} \frac{\delta S}{\delta \gamma_{a b}}=\frac{1}{8 \pi G}\left(\Theta^{a b}-\Theta \gamma^{a b}\right)+\frac{2}{\sqrt{|\gamma|}} \frac{\delta S^{0}}{\delta \gamma_{a b}}
$$

\footnotetext{
${ }^{14}$ In the original papers Brown and York assumed that the leaves $\Sigma_{t}$ of the foliation of $D$ were orthogonal to ${ }^{3} B$ ('orthogonal boundaries assumption').
} 
defines a symmetric tensor field on the timelike boundary ${ }^{3} B$, and is called the surface stressenergy tensor. (Since our signature for $\gamma_{a b}$ on ${ }^{3} B$ is $(+,-,-)$ rather than $(-,+,+)$, we should define $\tau^{a b}$ with the extra minus sign, just according to Equation (1).) Its divergence with respect to the connection ${ }^{3} D_{e}$ on ${ }^{3} B$ determined by $\gamma_{a b}$ is proportional to the part $\gamma^{a b} T_{b c} v^{c}$ of the energymomentum tensor, and hence, in particular, $\tau^{a b}$ is divergence-free in vacuum. Therefore, if $\left({ }^{3} B, \gamma_{a b}\right)$ admits a Killing vector, say $K^{a}$, then in vacuum

$$
Q_{\mathcal{S}}[\mathbf{K}]:=\oint_{\mathcal{S}} K_{a} \tau^{a b} \bar{t}_{b} d \mathcal{S}
$$

the flux integral of $\tau^{a b} K_{b}$ on any spacelike cross section $\mathcal{S}$ of ${ }^{3} B$, is independent of the cross section itself, and hence defines a conserved charge. If $K^{a}$ is timelike, then the corresponding charge is called a conserved mass, while for spacelike $K^{a}$ with closed orbits in $\mathcal{S}$ the charge is called angular momentum. (Here $\mathcal{S}$ is not necessarily an element of the foliation $\mathcal{S}_{t}$ of ${ }^{3} B$, and $\bar{t}^{a}$ is the unit normal to $\mathcal{S}$ tangent to ${ }^{3} B$.)

Clearly, the trace- $\chi$ action cannot be recovered as the volume integral of some scalar Lagrangian, because it is the Hilbert action plus a boundary integral of the trace $\chi$, and the latter depends on the location of the boundary itself. Such a Lagrangian was found by Pons [317]. This depends on the coordinate system adapted to the boundary of the domain $D$ of integration. An interesting feature of this Lagrangian is that it is second order in the derivatives of the metric, but it depends only on the first time derivative. A detailed analysis of the variational principle, the boundary conditions and the conserved charges is given. In particular, the asymptotic properties of this Lagrangian is similar to that of the ГГ Lagrangian of Einstein, rather than to that of Hilbert's.

\subsubsection{The general form of the Brown-York quasi-local energy}

The $3+1$ decomposition of the spacetime metric yields a $2+1$ decomposition of the metric $\gamma_{a b}$, too. Let $N$ and $N^{a}$ be the lapse and the shift of this decomposition on ${ }^{3} B$. Then the corresponding decomposition of $\tau^{a b}$ defines the energy, momentum, and spatial stress surface densities according to

$$
\begin{aligned}
\varepsilon & :=t_{a} t_{b} \tau^{a b}=-\frac{1}{8 \pi G} k+\frac{1}{\sqrt{|q|}} \frac{\delta S^{0}}{\delta N}, \\
j_{a} & :=-q_{a b} t_{c} \tau^{b c}=\frac{1}{8 \pi G} A_{a}+\frac{1}{\sqrt{|q|}} \frac{\delta S^{0}}{\delta N^{a}}, \\
s^{a b} & :=\Pi_{c}^{a} \Pi_{d}^{b} \tau^{c d}=\frac{1}{8 \pi G}\left[k^{a b}-k q^{a b}+q^{a b} t^{e}\left(\nabla_{e} t_{f}\right) v^{f}\right]+\frac{2}{\sqrt{|q|}} \frac{\delta S^{0}}{\delta q_{a b}},
\end{aligned}
$$

where $q_{a b}$ is the spacelike 2-metric, $A_{e}$ is the $S O(1,1)$ vector potential on $\mathcal{S}_{t}$ and $\Pi_{b}^{a}$ is the projection to $\mathcal{S}_{t}$ introduced in Section 4.1.2, and $k_{a b}$ is the extrinsic curvature of $\mathcal{S}_{t}$ corresponding to the normal $v^{a}$ orthogonal to ${ }^{3} B$, and $k$ is its trace. The timelike boundary ${ }^{3} B$ defines a boost-gauge on the 2-surfaces $\mathcal{S}_{t}$ (which coincides with that determined by the foliation $\Sigma_{t}$ in the 'orthogonal boundaries' case). The gauge potential $A_{e}$ is taken in this gauge. Thus, although $\varepsilon$ and $j_{a}$ on $\mathcal{S}_{t}$ are built from the 2-surface data (in a particular boost-gauge), the spatial surface stress depends on the part $t^{a}\left(\nabla_{a} t_{b}\right) v^{b}$ of the acceleration of the foliation $\Sigma_{t}$ too. Let $\xi^{a}$ be any vector field on ${ }^{3} B$ tangent to ${ }^{3} B$, and $\xi^{a}=n t^{a}+n^{a}$ its $2+1$ decomposition. Then we can form the charge integral (68) for the leaves $\mathcal{S}_{t}$ of the foliation of ${ }^{3} B$

$$
E_{t}\left[\xi^{a}, t^{a}\right]:=\oint_{\mathcal{S}_{t}} \xi_{a} \tau^{a b} t_{b} d \mathcal{S}_{t}=\oint_{\mathcal{S}_{t}}\left(n \varepsilon-n^{a} j_{a}\right) d \mathcal{S}_{t} .
$$

Living Reviews in Relativity

http: //www. livingreviews.org/Irr-2004-4 
Obviously, in general $E_{t}\left[\xi^{a}, t^{a}\right]$ is not conserved, and depends not only on the vector field $\xi^{a}$ and the 2-surface data on the particular $\mathcal{S}_{t}$, but on the boost-gauge that ${ }^{3} B$ defines on $\mathcal{S}_{t}$, i.e. the timelike normal $t^{a}$ as well. Brown and York define the general form of their quasi-local energy on $\mathcal{S}:=\mathcal{S}_{t}$ by

$$
E_{\mathrm{BY}}\left(\mathcal{S}, t^{a}\right):=E_{t}\left[t^{a}, t^{a}\right],
$$

i.e. they link the 'quasi-time-translation' (i.e. the 'generator of the energy') to the preferred unit normal $t^{a}$ of $\mathcal{S}_{t}$. Since the preferred unit normals $t^{a}$ are usually interpreted as a fleet of observers who are at rest with respect to $\mathcal{S}_{t}$, in their spirit the Brown-York-type quasi-local energy expressions are similar to $E_{\Sigma}\left[t^{a}\right]$ given by Equation (6) for the matter fields or Equation (18) for the gravitational 'field' rather than to the charges $Q_{\mathcal{S}}[\mathbf{K}]$. For vector fields $\xi^{a}=n^{a}$ with closed integral curved in $\mathcal{S}_{t}$ the quantity $E_{t}\left[\xi^{a}, t^{a}\right]$ might be interpreted as angular momentum corresponding to $\xi^{a}$.

The quasi-local energy is still not completely determined, because the 'subtraction term' $S^{0}$ in the principal function has not been specified. This term is usually interpreted as our freedom to shift the zero point of the energy. Thus the basic idea of fixing the subtraction term is to choose a 'reference configuration', i.e. a spacetime in which we want to obtain zero quasi-local quantities $E_{t}\left[\xi^{a}, t^{a}\right]$ (in particular zero quasi-local energy), and identify $S^{0}$ with the $S^{1}$ of the reference spacetime. Thus by Equation (69) and (70) we obtain that

$$
\varepsilon=-\frac{1}{8 \pi G}\left(k-k^{0}\right), \quad j_{a}=\frac{1}{8 \pi G}\left(A_{a}-A_{a}^{0}\right),
$$

where $k^{0}$ and $A_{a}^{0}$ are the reference values of the trace of the extrinsic curvature and $S O(1,1)$ gauge potential, respectively. Note that to ensure that $k^{0}$ and $A_{a}^{0}$ really be the trace of the extrinsic curvature and $S O(1,1)$-gauge potential, respectively, in the reference spacetime, they cannot depend on the lapse $N$ and the shift $N^{a}$. This can be ensured by requiring that $S^{0}$ be a linear functional of them. We return to the discussion of the reference term in the various specific constructions below.

\subsubsection{Further properties of the general expressions}

As we noted, $\varepsilon, j_{a}$, and $s_{a b}$ depend on the boost-gauge that the timelike boundary defines on $\mathcal{S}_{t}$. Lau clarified how these quantities change under a boost gauge transformation, where the new boost-gauge is defined by the timelike boundary ${ }^{3} B^{\prime}$ of another domain $D^{\prime}$ such that the particular 2-surface $\mathcal{S}_{t}$ is a leaf of the foliation of ${ }^{3} B^{\prime}$ too [247]: If $\left\{\bar{\Sigma}_{t}\right\}$ is another foliation of $D$ such that $\partial \bar{\Sigma}_{t}=\mathcal{S}_{t}$ and $\bar{\Sigma}_{t}$ is orthogonal to ${ }^{3} B$, then the new $\varepsilon^{\prime}, j_{a}^{\prime}$, and $s_{a b}^{\prime}$ are built from the old $\varepsilon, j_{a}$, and $s_{a b}$ and the $2+1$ pieces on $\mathcal{S}_{t}$ of the canonical momentum $\overline{\tilde{p}}^{a b}$, defined on $\bar{\Sigma}_{t}$. Apart from the contribution of $S^{0}$, these latter quantities are

$$
\begin{aligned}
& j_{\vdash}:=\frac{2}{\sqrt{|h|}} v_{a} v_{b} \overline{\tilde{p}}^{a b}=\frac{1}{8 \pi G} l, \\
& \hat{j}_{a}:=\frac{2}{\sqrt{|h|}} q_{a b} v_{c} \overline{\tilde{p}}^{c c}=\frac{1}{8 \pi G} A_{a}, \\
& t_{a b}:=\frac{2}{\sqrt{|h|}} q_{a c} q_{b d} \overline{\tilde{p}}^{c d}=\frac{1}{8 \pi G}\left[l_{a b}-q_{a b}\left(l+v^{e}\left(\nabla_{e} v_{f}\right) t^{e}\right)\right],
\end{aligned}
$$

where $l_{a b}$ is the extrinsic curvature of $\mathcal{S}_{t}$ corresponding to its normal $t^{a}$ (we denoted this by $\tau_{a b}$ in Section 4.1.2), and $l$ is its trace. (By Equation (76) $\hat{j}_{a}$ is not an independent quantity, that is just $j_{a}$. These quantities were originally introduced as the variational derivatives of the principal function with respect to the lapse, the shift and the 2-metric of the radial foliation of $\Sigma_{t}$ [247, 95], 
which are, in fact, essentially the components of the canonical momentum.) Thus the required transformation formulae for $\varepsilon, j_{a}$, and $s_{a b}$ follow from the definitions and those for the extrinsic curvatures and the $S O(1,1)$ gauge potential of Section 4.1.2. The various boost-gauge invariant quantities that can be built from $\varepsilon, j_{a}, s_{a b}, j_{\vdash}$, and $t_{a b}$ are also discussed in [247, 95].

Lau repeated the general analysis above using the tetrad (in fact, triad) variables and the Ashtekar connection on the timelike boundary instead of the traditional ADM-type variables [245]. Here the energy and momentum surface densities are re-expressed by the superpotential $\vee_{b}{ }^{a e}$, given by Equation (10), in a frame adapted to the 2-surface. (Lau called the corresponding superpotential 2-form the 'Sparling 2-form'.) However, in contrast to the usual Ashtekar variables on a spacelike hypersurface [17], the time gauge cannot be imposed globally on the boundary Ashtekar variables. In fact, while every orientable 3 -manifold $\Sigma$ is parallelizable [297], and hence a globally defined orthonormal triad can be given on $\Sigma$, the only parallelizable closed orientable 2 -surface is the torus. Thus, on ${ }^{3} B$, we cannot impose the global time gauge condition with respect to any spacelike 2surface $\mathcal{S}$ in ${ }^{3} B$ unless $\mathcal{S}$ is a torus. Similarly, the global radial gauge condition in the spacelike hypersurfaces $\Sigma_{t}$ (even on a small open neighbourhood of the whole 2-surfaces $\mathcal{S}_{t}$ in $\Sigma_{t}$ ) can be imposed on a triad field only if the 2-boundaries $\mathcal{S}_{t}=\partial \Sigma_{t}$ are all tori. Obviously, these gauge conditions can be imposed on every local trivialization domain of the tangent bundle $T \mathcal{S}_{t}$ of $\mathcal{S}_{t}$. However, since in Lau's local expressions only geometrical objects (like the extrinsic curvature of the 2-surface) appear, they are valid even globally (see also [246]). On the other hand, further investigations are needed to clarify whether or not the quasi-local Hamiltonian, using the Ashtekar variables in the radial-time gauge [247], is globally well-defined.

In general the Brown-York quasi-local energy does not have any positivity property even if the matter fields satisfy the dominant energy conditions. However, as G. Hayward pointed out [179], for the variations of the metric around the vacuum solutions that extremalize the Hamiltonian, called the 'ground states', the quasi-local energy cannot decrease. On the other hand, the interpretation of this result as a 'quasi-local dominant energy condition' depends on the choice of the time gauge above, which does not exist globally on the whole 2 -surface $\mathcal{S}$.

Booth and Mann [77] shifted the emphasis from the foliation of the domain $D$ to the foliation of the boundary ${ }^{3} B$. (These investigations were extended to include charged black holes in [78], where the gauge dependence of the quasi-local quantities is also examined.) In fact, from the point of view of the quasi-local quantities defined with respect to the observers with world lines in ${ }^{3} B$ and orthogonal to $\mathcal{S}$ it is irrelevant how the spacetime domain $D$ is foliated. In particular, the quasilocal quantities cannot depend on whether or not the leaves $\Sigma_{t}$ of the foliation of $D$ are orthogonal to ${ }^{3} B$. As a result, they recovered the quasi-local charge and energy expressions of Brown and York derived in the 'orthogonal boundary' case. However, they suggested a new prescription for the definition of the reference configuration (see Section 10.1.8). Also, they calculated the quasi-local energy for round spheres in the spherically symmetric spacetimes with respect to several moving observers, i.e., in contrast to Equation (73), they did not link the generator vector field $\xi^{a}$ to the normal $t^{a}$ of $\mathcal{S}_{t}$. In particular, the world lines of the observers are not integral curves of $(\partial / \partial t)$ in the coordinate basis given in Section 4.2.1 on the round spheres.

Using an explicit, non-dynamical background metric $g_{a b}^{0}$, one can construct a covariant, first order Lagrangian $L\left(g_{a b}, g_{a b}^{0}\right)$ for general relativity [230], and one can use the action $I_{D}\left[g_{a b}, g_{a b}^{0}\right]$ based on this Lagrangian instead of the trace $\chi$ action. Fatibene, Ferraris, Francaviglia, and Raiteri [135] clarified the relationship between the two actions, $I_{D}\left[g_{a b}\right]$ and $I_{D}\left[g_{a b}, g_{a b}^{0}\right]$, and the corresponding quasi-local quantities: Considering the reference term $S^{0}$ in the Brown-York expression as the action of the background metric $g_{a b}^{0}$ (which is assumed to be a solution of the field equations), they found that the two first order actions coincide if the spacetime metrics $g_{a b}$ and $g_{a b}^{0}$ coincide on the boundary $\partial D$. Using $L\left(g_{a b}, g_{a b}^{0}\right)$, they construct the conserved Noether current for any vector field $\xi^{a}$ and, by taking its flux integral, define charge integrals $Q_{\mathcal{S}}\left[\xi^{a}, g_{a b}, g_{a b}^{0}\right]$ on 2-surfaces

Living Reviews in Relativity

http://www. livingreviews.org//rr-2004-4 
$\mathcal{S}^{15}$. Again, the Brown-York quasi-local quantity $E_{t}\left[\xi^{a}, t^{a}\right]$ and $Q_{\mathcal{S}_{t}}\left[\xi^{a}, g_{a b}, g_{a b}^{0}\right]$ coincide if the spacetime metrics coincide on the boundary $\partial D$ and $\xi^{a}$ has some special form. Therefore, although the two approaches are basically equivalent under the boundary condition above, this boundary condition is too strong both from the points of view of the variational principle and the quasi-local quantities. We will see in Section 10.1.8 that even the weaker boundary condition that only the induced 3-metrics on ${ }^{3} B$ from $g_{a b}$ and from $g_{a b}^{0}$ be the same is still too strong.

\subsubsection{The Hamiltonians}

If we can write the action $I[q(t)]$ of our mechanical system into the canonical form $\int_{t_{1}}^{t_{2}}\left[p_{a} \dot{q}^{a}-\right.$ $\left.H\left(q^{a}, p_{a}, t\right)\right] d t$, then it is straightforward to read off the Hamiltonian of the system. Thus, having accepted the trace $\chi$ action as the action for general relativity, it is natural to derive the corresponding Hamiltonian in the analogous way. Following this route Brown and York derived the Hamiltonian, corresponding to the 'basic' (or non-referenced) action $I^{1}$ too [97]. They obtained the familiar integral of the sum of the Hamiltonian and the momentum constraints, weighted by the lapse $N$ and the shift $N^{a}$, respectively, plus $E_{t}\left[N t^{a}+N^{a}, t^{a}\right]$, given by Equation (72), as a boundary term. This result is in complete agreement with the expectations, as their general quasilocal quantities can also be recovered as the value of the Hamiltonian on the constraint surface (see also [77]). This Hamiltonian was investigated further in [95]. Here all the boundary terms that appear in the variation of their Hamiltonian are determined and decomposed with respect to the 2 -surface $\partial \Sigma$. It is shown that the change of the Hamiltonian under a boost of $\Sigma$ yields precisely the boosts of the energy and momentum surface density discussed above.

Hawking, Horowitz, and Hunter also derived the Hamiltonian from the trace $\chi$ action $I_{D}^{1}\left[g_{a b}\right]$ both with the orthogonal [176] and non-orthogonal boundaries assumptions [177]. They allowed matter fields $\Phi_{N}$, whose dynamics is governed by a first order action $I_{\mathrm{m} D}^{1}\left[g_{a b}, \Phi_{N}\right]$, to be present. However, they treated the reference configuration in a different way. In the traditional canonical analysis of the fields and the geometry based on a non-compact $\Sigma$ (for example in the asymptotically flat case) one has to impose certain fall-off conditions that ensure the finiteness of the action, the Hamiltonian, etc. This finiteness requirement excludes several potentially interesting field + gravity configurations from our investigations. In fact, in the asymptotically flat case we compare the actual matter + gravity configurations with the flat spacetime+vanishing matter fields configuration. Hawking and Horowitz generalized this picture by choosing a static, but otherwise arbitrary solution $g_{a b}^{0}, \Phi_{N}^{0}$ of the field equations, considered the timelike boundary ${ }^{3} B$ of $D$ to be a timelike cylinder 'near the infinity', and considered the action

$$
I_{D}\left[g_{a b}, \Phi_{N}\right]:=I_{D}^{1}\left[g_{a b}\right]+I_{\mathrm{m} D}^{1}\left[g_{a b}, \Phi_{N}\right]-I_{D}^{1}\left[g_{a b}^{0}\right]-I_{\mathrm{m} D}^{1}\left[g_{a b}^{0}, \Phi_{N}^{0}\right]
$$

and those matter + gravity configurations which induce the same value on ${ }^{3} B$ as $\Phi_{N}^{0}$ and $g_{a b}^{0}$. Its limit as ${ }^{3} B$ is 'pushed out to infinity' can be finite even if the limit of the original (i.e. nonreferenced) action is infinite. Although in the non-orthogonal boundaries case the Hamiltonian derived from the non-referenced action contains terms coming from the 'joints', by the boundary conditions at ${ }^{3} B$ they are canceled from the referenced Hamiltonian. This latter Hamiltonian coincides with that obtained in the orthogonal boundaries case. Both the ADM and the AbbottDeser energy can be recovered from this Hamiltonian [176], and the quasi-local energy for spheres in domains with non-orthogonal boundaries in the Schwarzschild solution is also calculated [177]. A similar Hamiltonian, including the 'joints' or 'corner' terms, was obtained by Francaviglia and Raiteri [141] for the vacuum Einstein theory (and for Einstein-Maxwell systems in [4]), using a Noether charge approach. Their formalism, using the language of jet bundles, is, however, slightly more sophisticated than that common in general relativity.

\footnotetext{
${ }^{15}$ The paper gives a clear, well readable summary of these earlier results.
} 
Booth and Fairhurst [73] reexamined the general form of the Brown-York energy and angular momentum from a Hamiltonian point of view ${ }^{16}$. Their starting point is the observation that the domain $D$ is not isolated from its environment, thus the quasi-local Hamiltonian cannot be time independent. Therefore, instead of the standard Hamiltonian formalism for the autonomous systems, a more general formalism, based on the extended phase space, must be used. This phase space consists of the usual bulk configuration and momentum variables $\left(h_{a b}, \tilde{p}^{a b}\right)$ on the typical 3 -manifold $\Sigma$ and the time coordinate $t$, the space coordinates $x^{A}$ on the 2 -boundary $\mathcal{S}=\partial \Sigma$, and their conjugate momenta $\pi$ and $\pi_{A}$, respectively.

Their second important observation is that the Brown-York boundary conditions are too restrictive: The 2-metric, the lapse, and the shift need not to be fixed but their variations corresponding to diffeomorphisms on the boundary must be allowed. Otherwise diffeomorphisms that are not isometries of the 3 -metric $\gamma_{a b}$ on ${ }^{3} B$ cannot be generated by any Hamiltonian. Relaxing the boundary conditions appropriately, they show that there is a Hamiltonian on the extended phase space which generates the correct equations of motions, and the quasi-local energy and angular momentum expression of Brown and York are just (minus) the momentum $\pi$ conjugate to the time coordinate $t$. The only difference between the present and the original Brown-York expressions is the freedom in the functional form of the unspecified reference term: Because of the more restrictive boundary conditions of Brown and York their reference term is less restricted. Choosing the same boundary conditions in both approaches the resulting expressions coincide completely.

\subsubsection{The flat space and light cone references}

The quasi-local quantities introduced above become well-defined only if the subtraction term $S^{0}$ in the principal function is specified. The usual interpretation of a choice for $S^{0}$ is the calibration of the quasi-local quantities, i.e. fixing where to take their zero value.

The only restriction on $S^{0}$ that we had is that it must be a functional of the metric $\gamma_{a b}$ on the timelike boundary ${ }^{3} B$. To specify $S^{0}$, it seems natural to expect that the principal function $S$ be zero in Minkowski spacetime [158, 96]. Then $S^{0}$ would be the integral of the trace $\Theta^{0}$ of the extrinsic curvature of ${ }^{3} B$ if it were embedded in Minkowski spacetime with the given intrinsic metric $\gamma_{a b}$. However, a general Lorentzian 3-manifold $\left({ }^{3} B, \gamma_{a b}\right)$ cannot be isometrically embedded, even locally, into the Minkowski spacetime. (For a detailed discussion of this embeddability, see [96] and Section 10.1.8.)

Another assumption on $S^{0}$ might be the requirement of the vanishing of the quasi-local quantities, or of the energy and momentum surface densities, or only of the energy surface density $\varepsilon$, in some reference spacetime, e.g. in Minkowski or in anti-de-Sitter spacetime. Assuming that $S^{0}$ depends on the lapse $N$ and shift $N^{a}$ linearly, the functional derivatives $\left(\delta S^{0} / \delta N\right)$ and $\left(\delta S^{0} / \delta N^{a}\right)$ depend only on the 2-metric $q_{a b}$ and on the boost-gauge that ${ }^{3} B$ defined on $\mathcal{S}_{t}$. Therefore, $\varepsilon$ and $j_{a}$ take the form (74), and by the requirement of the vanishing of $\varepsilon$ in the reference spacetime it follows that $k^{0}$ should be the trace of the extrinsic curvature of $\mathcal{S}_{t}$ in the reference spacetime. Thus it would be natural to fix $k^{0}$ as the trace of the extrinsic curvature of $\mathcal{S}_{t}$ when $\left(\mathcal{S}_{t}, q_{a b}\right)$ is embedded isometrically into the reference spacetime. However, this embedding is far from being unique (since, in particular, there are two independent normals of $\mathcal{S}_{t}$ in the spacetime and it would not be fixed which normal should be used to calculate $k^{0}$ ), and hence the construction would be ambiguous. On the other hand, one could require $\left(\mathcal{S}_{t}, q_{a b}\right)$ to be embedded into flat Euclidean 3-space, i.e. into a spacelike hyperplane of Minkowski spacetime ${ }^{17}$. This is the choice of Brown and York [96, 97]. In fact, at least for a large class of 2 -surfaces $\left(\mathcal{S}_{t}, q_{a b}\right)$, such an embedding exists and

\footnotetext{
${ }^{16}$ Thus, in principle, we would have to report on their investigations in the next Section 11 . Nevertheless, since essentially they re-derive and justify the results of Brown and York following only a different route, we discuss their results here.

${ }^{17}$ The problem to characterize this embeddability is known as the Weyl problem of differential geometry.
}

Living Reviews in Relativity

http://www. livingreviews.org//rr-2004-4 
is unique: If $\mathcal{S}_{t} \approx S^{2}$ and the metric is $C^{2}$ and has everywhere positive scalar curvature, then there is an isometric embedding of $\left(\mathcal{S}_{t}, q_{a b}\right)$ into the flat Euclidean 3-space [195], and apart from rigid motions this embedding is unique [346]. The requirement that the scalar curvature of the 2-surface must be positive can be interpreted as some form of the convexity, as in the theory of surfaces in the Euclidean space. However, there are counterexamples even to local isometric embeddability when this convexity condition is violated [276]. A particularly interesting 2-surface that cannot be isometrically embedded into the flat 3-space is the event horizon of the Kerr black hole if the angular momentum parameter $a$ exceeds the irreducible mass (but is still not greater than the mass parameter $m$ ), i.e. if $\sqrt{3} m<2|a|<2 m$ [343]. Thus, the construction works for a large class of 2 -surfaces, but certainly not for every potentially interesting 2 -surface. The convexity condition is essential.

It is known that the (local) isometric embeddability of $\left(\mathcal{S}, q_{a b}\right)$ into flat 3-space with extrinsic curvature $k_{a b}^{0}$ is equivalent to the Gauss-Codazzi-Mainardi equations $\delta_{a}\left(k^{0 a}{ }_{b}-\delta_{b}^{a} k^{0}\right)=0$ and ${ }^{\mathcal{S}} R-\left(k^{0}\right)^{2}+k_{a b}^{0} k^{0 a b}=0$. Here $\delta_{a}$ is the intrinsic Levi-Civita covariant derivative and ${ }^{\mathcal{S}} R$ is the corresponding curvature scalar on $\mathcal{S}$ determined by $q_{a b}$. Thus, for given $q_{a b}$ and (actually the flat) embedding geometry, these are three equations for the three components of $k_{a b}^{0}$, and hence, if the embedding exists, $q_{a b}$ determines $k^{0}$. Therefore, the subtraction term $k^{0}$ can also be interpreted as a solution of an under-determined elliptic system which is constrained by a nonlinear algebraic equation. In this form the definition of the reference term is technically analogous to the definition of those in Sections 7, 8, and 9, but, by the non-linearity of the equations, in practice it is much more difficult to find the reference term $k^{0}$ than the spinor fields in the constructions of Sections 7 , 8 , and 9 .

Accepting this choice for the reference configuration, the reference $S O(1,1)$ gauge potential $A_{a}^{0}$ will be zero in the boost-gauge in which the timelike normal of $\mathcal{S}_{t}$ in the reference Minkowski spacetime is orthogonal to the spacelike 3-plane, because this normal is constant. Thus, to summarize, for convex 2-surfaces the flat space reference of Brown and York is uniquely determined, $k^{0}$ is determined by this embedding, and $A_{a}^{0}=0$. Then $8 \pi G S^{0}=-\int_{\mathcal{S}_{t}} N k^{0} d \mathcal{S}_{t}$, from which $s^{a b}$ can be calculated (if needed). The procedure is similar if, instead of a spacelike hyperplane of Minkowski spacetime, a spacelike hypersurface of constant curvature (for example in the de-Sitter or anti-de-Sitter spacetime) is used. The only difference is that extra (known) terms appear in the Gauss-Codazzi-Mainardi equations.

Brown, Lau, and York considered another prescription for the reference configuration as well [94, $248,249]$. In this approach the 2 -surface $\left(\mathcal{S}_{t}, q_{a b}\right)$ is embedded into the light cone of a point of the Minkowski or anti-de Sitter spacetime instead of a spacelike hypersurface of constant curvature. The essential difference between the new ('light cone reference') and the previous ('flat space reference') prescriptions is that the embedding into the light cone is not unique, but the reference term $k^{0}$ may be given explicitly, in a closed form. The positivity of the Gauss curvature of the intrinsic geometry of $\left(\mathcal{S}, q_{a b}\right)$ is not needed. In fact, by a result of Brinkmann [91], every locally conformally flat Riemannian $n$-geometry is locally isometric to an appropriate cut of a light cone of the $n+2$ dimensional Minkowski spacetime (see also [133]). To achieve uniqueness some extra condition must be imposed. This may be the requirement of the vanishing of the 'normal momentum density' $j_{\vdash}^{0}$ in the reference spacetime [248, 249], yielding $k^{0}=\sqrt{2^{\mathcal{S}} R+4 / \lambda^{2}}$, where ${ }^{\mathcal{S}} R$ is the Ricci scalar of $\left(\mathcal{S}, q_{a b}\right)$ and $\lambda$ is the cosmological constant of the reference spacetime. The condition $j_{\vdash}^{0}=0$ defines something like a 'rest frame' in the reference spacetime. Another, considerably more complicated choice for the light cone reference term is used in [94].

\subsubsection{Further properties and the various limits}

Although the general, non-referenced expressions are additive, the prescription for the reference term $k^{0}$ destroys the additivity in general. In fact, if $\mathcal{S}^{\prime}$ and $\mathcal{S}^{\prime \prime}$ are 2-surfaces such that $\mathcal{S}^{\prime} \cap \mathcal{S}^{\prime \prime}$ 
is connected and 2-dimensional (more precisely, it has a non-empty open interior for example in $\mathcal{S}^{\prime}$ ), then in general $\overline{\mathcal{S}^{\prime} \cup \mathcal{S}^{\prime \prime}-\mathcal{S}^{\prime} \cap \mathcal{S}^{\prime \prime}}$ (overline means topological closure) is not guaranteed to be embeddable into the flat 3-space, and even if it is embeddable then the resulting reference term $k^{0}$ differs from the reference terms $k^{\prime 0}$ and $k^{\prime \prime 0}$ determined from the individual embeddings.

As it is noted in [77], the Brown-York energy with the flat space reference configuration is not zero in Minkowski spacetime in general. In fact, in the standard spherical polar coordinates let $\Sigma_{1}$ be the spacelike hyperboloid $t=-\sqrt{\rho^{2}+r^{2}}, \Sigma_{0}$ the hyperplane $t=-T=$ const. $<-\rho<0$ and $\mathcal{S}:=\Sigma_{0} \cap \Sigma_{1}$, the sphere of radius $\sqrt{T^{2}-\rho^{2}}$ in the $t=-T$ hyperplane. Then the trace of the extrinsic curvature of $\mathcal{S}$ in $\Sigma_{0}$ and in $\Sigma_{1}$ is $2 / \sqrt{T^{2}-\rho^{2}}$ and $2 T / \rho \sqrt{T^{2}-\rho^{2}}$, respectively. Therefore, the Brown-York quasi-local energy (with the flat 3-space reference) associated with $\mathcal{S}$ and the normals of $\Sigma_{1}$ on $\mathcal{S}$ is $-\sqrt{(T+\rho)(T-\rho)^{3}} /(\rho G)$. Similarly, the Brown-York quasi-local energy with the light cone references in [248] and in [94] is also negative for such surfaces with the boosted observers.

Recently, Shi, and Tam [341] proved interesting theorems in Riemannian 3-geometries, which can be used to prove positivity of the Brown-York energy if the 2-surface $\mathcal{S}$ is a boundary of some time-symmetric spacelike hypersurface on which the dominant energy condition holds. In the time-symmetric case this energy condition is just the condition that the scalar curvature be non-negative. The key theorem of Shi and Tam is the following: Let $\Sigma$ be a compact, smooth Riemannian 3-manifold with non-negative scalar curvature and smooth 2-boundary $\mathcal{S}$ such that each connected component $\mathcal{S}_{i}$ of $\mathcal{S}$ is homeomorphic to $S^{2}$ and the scalar curvature of the induced 2 -metric on $\mathcal{S}_{i}$ is strictly positive. Then for each component $\oint_{\mathcal{S}_{i}} k d \mathcal{S}_{i} \leq \oint_{\mathcal{S}_{i}} k^{0} d \mathcal{S}_{i}$ holds, where $k$ is the trace of the extrinsic curvature of $\mathcal{S}$ in $\Sigma$ with respect to the outward directed normal, and $k^{0}$ is the trace of the extrinsic curvature of $\mathcal{S}_{i}$ in the flat Euclidean 3-space when $\mathcal{S}_{i}$ is isometrically embedded. Furthermore, if in these inequalities the equality holds for at least one $\mathcal{S}_{i}$, then $\mathcal{S}$ itself is connected and $\Sigma$ is flat. This result is generalized in [342] by weakening the energy condition, whenever lower estimates of the Brown-York energy can still be given.

The energy expression for round spheres in spherically symmetric spacetimes was calculated in $[97,77]$. In the spherically symmetric metric discussed in Section 4.2.1, on the round spheres the Brown-York energy with the flat space reference and fleet of observers $\partial / \partial t$ on $\mathcal{S}$ is $G E_{\mathrm{BY}}\left[\mathcal{S}_{r},(\partial / \partial t)^{a}\right]=$ $r(1-\exp (-\alpha))$. In particular, it is $r[1-\sqrt{1-(2 m / r)}]$ for the Schwarzschild solution. This deviates from the standard round sphere expression, and, for the horizon of the Schwarzschild black hole it is $2 m$ (instead of the expected $m$ ). (The energy has also been calculated explicitly for boosted foliations of the Schwarzschild solution and for round spheres in isotropic cosmological models [95].) The Newtonian limit can be derived from this by assuming that $m$ is the mass of a fluid ball of radius $r$ and $m / r$ is small: It is $G E_{\mathrm{BY}}=m+\left(m^{2} / 2 r\right)+\mathcal{O}\left(r^{-2}\right)$. The first term is simply the mass defined at infinity, and the second term is minus the Newtonian potential energy associated with building a spherical shell of mass $m$ and radius $r$ from individual particles, bringing them together from infinity. However, taking into account that on the Schwarzschild horizon $G E_{\mathrm{BY}}=2 m$ while at the spatial infinity it is just $m$, the Brown-York energy is monotonically decreasing with $r$. Also, the first law of black hole mechanics for spherically symmetric black holes can be recovered by identifying $E_{\mathrm{BY}}$ with the internal energy [96, 97]. The thermodynamics of the Schwarzschild-anti-de-Sitter black holes was investigated in terms of the quasi-local quantities in [92]. Still considering $E_{\mathrm{BY}}$ to be the internal energy, the temperature, surface pressure, heat capacity, etc. are calculated (see Section 13.3.1). The energy has also been calculated for the Einstein-Rosen cylindrical waves [95].

The energy is explicitly calculated for three different kinds of 2 -spheres in the $t=$ const. slices (in the Boyer-Lindquist coordinates) of the slow rotation limit of the Kerr black hole spacetime with the flat space reference [264]. These surfaces are the $r=$ const. surfaces (such as the outer horizon), spheres whose intrinsic metric (in the given slow rotation approximation) is of a metric sphere of radius $R$ with surface area $4 \pi R^{2}$, and the ergosurface (i.e. the outer boundary of the

Living Reviews in Relativity

http: //www. livingreviews . org//rr-2004-4 
ergosphere). The slow rotation approximation is defined such that $|a| / R \ll 1$, where $R$ is the typical spatial measure of the 2 -surface. In the first two cases the angular momentum parameter $a$ enters the energy expression only in the $m^{2} a^{2} / R^{3}$ order. In particular, the energy for the outer horizon $r_{+}:=m+\sqrt{m^{2}-a^{2}}$ is $2 m\left[1-a^{2} /\left(8 m^{2}\right)+\mathcal{O}\left(a^{4} / r_{+}^{4}\right)\right]$, which is twice the irreducible mass of the black hole. An interesting feature of this calculation is that the energy cannot be calculated for the horizon directly, because, as we noted in the previous point, the horizon itself cannot be isometrically embedded into a flat 3 -space if the angular momentum parameter exceeds the irreducible mass [343]. The energy for the ergosurface is positive, as for the other two kinds of surfaces.

The spacelike infinity limit of the charges interpreted as the energy, spatial momentum, and spatial angular momentum are calculated in [95] (see also [176]). Here the flat space reference configuration and the asymptotic Killing vectors of the spacetime are used, and the limits coincide with the standard ADM energy, momentum, and spatial angular momentum. The analogous calculation for the centre-of-mass is given in [42]. It is shown that the corresponding large sphere limit is just the centre-of-mass expression of Beig and Ó Murchadha [47]. Here the centre-of-mass integral in terms of a charge integral of the curvature is also given.

Although the prescription for the reference configuration by Hawking and Horowitz cannot be imposed for a general timelike 3-boundary ${ }^{3} B$ (see Section 10.1.8), asymptotically, when ${ }^{3} B$ is pushed out to infinity, this prescription can be used, and coincides with the prescription of Brown and York. Choosing the background metric $g_{a b}^{0}$ to be the anti-de-Sitter one, Hawking and Horowitz [176] calculated the limit of the quasi-local energy, and they found it to tend to the Abbott-Deser energy. (For the spherically symmetric, Schwarzschild-anti-de-Sitter case see also [92].) In [93] the null infinity limit of the integral of $N\left(k^{0}-k\right) /(8 \pi G)$ was calculated both for the lapses $N$ generating asymptotic time translations and supertranslations at the null infinity, and the fleet of observers was chosen to tend to the BMS translation. In the former case the Bondi-Sachs energy, in the latter case Geroch's supermomenta are recovered. These calculations are based directly on the Bondi form of the spacetime metric, and do not use the asymptotic solution of the field equations. In a slightly different formulation Booth and Creighton calculated the energy flux of outgoing gravitational radiation [76] (see also Section 13.1) and they recovered the Bondi-Sachs mass-loss.

However, the calculation of the small sphere limit based on the flat space reference configuration gave strange results [249]. While in non-vacuum the quasi-local energy is the expected $(4 \pi / 3) r^{3} T_{a b} t^{a} t^{b}$, in vacuum it is proportional to $4 E_{a b} E^{a b}+H_{a b} H^{a b}$ instead of the Bel-Robinson 'energy' $T_{a b c d} t^{a} t^{b} t^{c} t^{d}$. (Here $E_{a b}$ and $H_{a b}$ are, respectively, the conformal electric and conformal magnetic curvatures, and $t^{a}$ plays a double role: It defines the 2-sphere of radius $r$ [as is usual in the small sphere calculations], and defines the fleet of observers on the 2-sphere.) On the other hand, the special light cone reference used in [94, 249] reproduces the expected result in non-vacuum, and yields $[1 /(90 G)] r^{5} T_{a b c d} t^{a} t^{b} t^{c} t^{d}$ in vacuum.

The light cone reference $k^{0}=\sqrt{2^{\mathcal{S}} R+4 / \lambda^{2}}$ was shown to work in the large sphere limit near the null and spatial infinities of asymptotically flat, and near the infinity of asymptotically antide-Sitter spacetimes [248]. Namely, the Brown-York quasi-local energy expression with this null cone reference term tends to the Bondi-Sachs, the ADM, and Abbott-Deser energies, respectively. The supermomenta of Geroch at null infinity can also be recovered in this way. The proof is simply a demonstration of the fact that this light cone and the flat space prescriptions for the subtraction term have the same asymptotic structure up to order $\mathcal{O}\left(r^{-3}\right)$. This choice seems to work properly only in the asymptotics, because for small ellipsoids in the Minkowski spacetime this definition yields non-zero energy and for small spheres in vacuum it does not yield the Bel-Robinson 'energy' [250]. 


\subsubsection{Other prescriptions for the reference configuration}

As we noted above, Hawking, Horowitz, and Hunter [176, 177] defined their reference configuration by embedding the Lorentzian 3-manifold $\left({ }^{3} B, \gamma_{a b}\right)$ isometrically into some given Lorentzian spacetime, e.g. into the Minkowski spacetime (see also [158]). However, for the given intrinsic 3-metric $\gamma_{a b}$ and the embedding 4-geometry the corresponding Gauss and Codazzi-Mainardi equations form a system of $6+8=14$ equations for the six components of the extrinsic curvature $\Theta_{a b}$ [96]. Thus, in general, this is a highly overdetermined system, and hence it may be expected to have a solution only in exceptional cases. However, even if such an embedding existed, then even the small perturbations of the intrinsic metric $h_{a b}$ would break the conditions of embeddability. Therefore, in general this prescription for the reference configuration can work only if the 3 -surface ${ }^{3} B$ is 'pushed out to infinity' but does not work for finite 3-surfaces [96].

To rule out the possibility that the Brown-York energy can be non-zero even in Minkowski spacetime (on 2-surfaces in the boosted flat data set), Booth and Mann [77] suggested to embed $\left(\mathcal{S}, q_{a b}\right)$ isometrically into a reference spacetime $\left(M^{0}, g_{a b}^{0}\right)$ (mostly into the Minkowski spacetime) instead of a spacelike slice of it, and to map the evolution vector field $\xi^{a}=N t^{a}+N^{a}$ of the dynamics, tangent to ${ }^{3} B$, to a vector field $\xi^{0 a}$ in $M^{0}$ such that $\mathrm{E}_{\xi} q_{a b}=\phi^{*}\left(\mathrm{E}_{\xi^{0}} q_{a b}^{0}\right)$ and $\xi^{a} \xi_{a}=$ $\phi^{*}\left(\xi^{0 a} \xi_{a}^{0}\right)$. Here $\phi$ is a diffeomorphism mapping an open neighbourhood $U$ of $\mathcal{S}$ in $M$ into $M^{0}$ such that $\left.\phi\right|_{\mathcal{S}}$, the restriction of $\phi$ to $\mathcal{S}$, is an isometry, and $\mathrm{E}_{\xi} q_{a b}$ denotes the Lie derivative of $q_{a b}$ along $\xi^{a}$. This condition might be interpreted as some local version of that of Hawking, Horowitz, and Hunter. However, Booth and Mann did not investigate the existence or the uniqueness of this choice.

\subsection{Kijowski's approach}

\subsubsection{The role of the boundary conditions}

In the Brown-York approach the leading principle was the claim to have a well-defined variational principle. This led them to modify the Hilbert action to the trace- $\chi$-action and the boundary condition that the induced 3-metric on the boundary of the domain $D$ of the action is fixed.

However, as stressed by Kijowski [237, 239], the boundary conditions have much deeper content. For example in thermodynamics the different definitions of the energy (internal energy, enthalpy, free energy, etc.) are connected with different boundary conditions. Fixing the pressure corresponds to enthalpy, but fixing the temperature to free energy. Thus the different boundary conditions correspond to different physical situations, and, mathematically, to different phase spaces ${ }^{18}$. Therefore, to relax the a priori boundary conditions, Kijowski abandoned the variational principle and concentrated on the equations of motions. However, to treat all possible boundary conditions on an equal footing he used the enlarged phase space of Tulczyjew (see for example [239]) ${ }^{19}$. The boundary condition of Brown and York is only one of the possible boundary conditions.

\subsubsection{The analysis of the Hilbert action and the quasi-local internal and free energies}

Starting with the variation of Hilbert's Lagrangian (in fact, the corresponding Hamilton-Jacobi principal function on a domain $D$ above), and defining the Hamiltonian by the standard Legendre transformation on the typical compact spacelike 3-manifold $\Sigma$ and its boundary $\mathcal{S}=\partial \Sigma$ too, Kijowski arrived at a variation formula involving the value on $\mathcal{S}$ of the variation of the canonical

\footnotetext{
${ }^{18}$ According to this view the quasi-local energy is similar to $E_{\Sigma}$ of Equation (6), rather than to the charges which are connected somehow to some 'absolute' element of the spacetime structure.

${ }^{19}$ This phase space is essentially $T^{*} T Q$, the cotangent bundle of the tangent bundle of the configuration manifold $Q$, endowed with the natural symplectic structure, and can be interpreted as the collection of quadruples $\left(q^{a}, \dot{q}^{a}, p_{a}, \dot{p}_{a}\right)$. The usual Lagrangian (or velocity) phase space $T Q$ and the Hamiltonian (or momentum) phase space $T^{*} Q$ are special submanifolds of $T^{*} T Q$.
}

Living Reviews in Relativity

http://www. livingreviews.org//rr-2004-4 
momentum, $\tilde{\pi}^{a b}:=-\frac{1}{16 \pi G} \sqrt{|\gamma|}\left(\Theta^{a b}-\Theta \gamma^{a b}\right)$, conjugate to $\gamma_{a b}$. (Apart from a numerical coefficient and the subtraction term, this is essentially the surface stress-energy tensor $\tau^{a b}$ given by Equation (67).) Since, however, it is not clear whether or not the initial + boundary value problem for the Einstein equations with fixed canonical momenta (i.e. extrinsic curvature) is well posed, he did not consider the resulting Hamiltonian as the appropriate one, and made further Legendre transformations on the boundary $\mathcal{S}$.

The first Legendre transformation that he considered gave a Hamiltonian whose variation involves the variation of the induced 2-metric $q_{a b}$ on $\mathcal{S}$ and the parts $\tilde{\pi}^{a b} t_{a} t_{b}$ and $\tilde{\pi}^{a b} t_{a} \Pi_{b}^{c}$ of the canonical momentum above. Explicitly, with the notations of the previous Section 10.1, the latter two are $\pi^{a b} t_{a} t_{b}=k /(16 \pi G)$ and $\pi^{a b} t_{a} q_{b c}=A_{c} /(16 \pi G)$, respectively. $\left(\pi^{a b}\right.$ is the de-densitized $\tilde{\pi}^{a b}$.) Then, however, the lapse and the shift on the boundary $\mathcal{S}$ will not be independent: As Kijowski shows they are determined by the boundary conditions for the 2-metric and the freely specifiable parts $k$ and $A_{c}$ of the canonical momentum $\pi^{a b}$. Then, to define the 'quasi-symmetries' of the 2-surface, Kijowski suggests to embed first the 2 -surface isometrically into an $x^{0}=$ const. hyperplane of the Minkowski spacetime, and then define a world tube by dragging this 2-surface along the integral curves of the Killing vectors of the Minkowski spacetime. For example, to define the 'quasi time translation' of the 2-surface in the physical spacetime we must consider the time translation in the Minkowski spacetime of the 2-surface embedded in the $x^{0}=$ const. hyperplane. This world tube gives an extrinsic curvature $k_{a b}^{0}$ and vector potential $A_{c}^{0}$. Finally, Kijowski's choice for $k$ and $A_{c}$ is just $k^{0}$ and $A_{c}^{0}$, respectively. In particular, to define the 'quasi time translation' he takes $\pi^{a b} t_{a} t_{b}=k^{0} /(16 \pi G)$ and $\pi^{a b} t_{a} \Pi_{b}^{c}=0$, because this choice yields zero shift and constant lapse with value 1 . The corresponding quasi-local quantity, the Kijowski energy, is

$$
E_{\mathrm{K}}(\mathcal{S}):=\frac{1}{16 \pi G} \oint_{\mathcal{S}} \frac{\left(k^{0}\right)^{2}-\left(k^{2}-l^{2}\right)}{k^{0}} d \mathcal{S} .
$$

Here, as above, $k$ and $l$ are the trace of the extrinsic curvatures of $\mathcal{S}$ in the physical spacetime corresponding to the outward pointing spacelike and the future pointing timelike unit normals to $\mathcal{S}$, which are orthogonal to each other. Obviously, $E_{\mathrm{K}}(\mathcal{S})$ is invariant with respect to the boost gauge transformations of the normals, because the 'generator vector field' of the energy is not linked to one of the normals of $\mathcal{S}$. A remarkable property of this procedure is that for round spheres in the Schwarzschild solution the choice $\pi^{a b} t_{a} t_{b}=k^{0} /(16 \pi G), \pi^{a b} t_{a} q_{b c}=0$ (i.e. the flat spacetime values) reproduces the lapse of the correct Schwarzschild time [237]. For round spheres (see Section 4.2.1) Equation (78) gives $\frac{r}{2 G}[1-\exp (-2 \alpha)]$, which is precisely the standard round sphere expression (27). In particular [237], for the event horizon of the Schwarzschild solution it gives the expected value $m / G$. However, there exist spacelike topological 2-spheres $\mathcal{S}$ in the Minkowski spacetime for which $E_{\mathrm{K}}(\mathcal{S})$ is positive [292].

Kijowski considered another Legendre transformation on the 2-surface too, and in the variation of the resulting Hamiltonian only the value on $\mathcal{S}$ of the variation of the metric $\gamma_{a b}$ appears. Thus in this phase space the components of $\gamma_{a b}$ can be specified freely on $\mathcal{S}$, and Kijowski calls the value of the resulting Hamiltonian the 'free energy'. Its form is

$$
F_{\mathrm{K}}(\mathcal{S}):=\frac{1}{8 \pi G} \oint_{\mathcal{S}}\left(k^{0}-\sqrt{k^{2}-l^{2}}\right) d \mathcal{S} .
$$

In the special boost-gauge when $l=0$ the 'free energy' $F_{\mathrm{K}}(\mathcal{S})$ reduces to the Brown-York expression $E_{\mathrm{BY}}(\mathcal{S})$ given by Equation $(73) . F_{\mathrm{K}}(\mathcal{S})$ appears to have been re-discovered recently by Liu and Yau [253], and we discuss the properties of $F_{\mathrm{K}}(\mathcal{S})$ further in Section 10.4. A more detailed discussion of the possible quasi-local Hamiltonians and the strategies to define the appropriate 'quasi-symmetries' of $\mathcal{S}$ are given in [238]. 


\subsection{The expression of Epp}

\subsubsection{The general form of Epp's expression}

The Brown-York energy expression, based on the original flat space reference, has the highly undesirable property that it gives non-zero energy even in the Minkowski spacetime if the fleet of observers on the spherical $\mathcal{S}$ is chosen to be radially accelerating (see the second paragraph in Section 10.1.7). Thus it would be a legitimate aim to reduce this extreme dependence of the quasilocal energy on the choice of the observers. One way of doing this is to formulate the quasi-local quantities in terms of boost-gauge invariant objects. Such a boost-gauge invariant geometric object is the length of the mean extrinsic curvature vector $Q^{a}$ of Section 4.1.2, which, in the notations of the present section, is $\sqrt{k^{2}-l^{2}}$. If $Q^{a}$ is spacelike or null, then this square root is real, and (apart from the reference term $k^{0}$ in Equation (73)) in the special case $l=0$ it reduces to $-8 \pi G$ times the surface energy density of Brown and York. This observation lead Epp to suggest

$$
E_{\mathrm{E}}(\mathcal{S}):=\frac{1}{8 \pi G} \oint_{\mathcal{S}}\left(\sqrt{\left(k^{0}\right)^{2}-\left(l^{0}\right)^{2}}-\sqrt{k^{2}-l^{2}}\right) d \mathcal{S}
$$

as the general definition of the so-called 'invariant quasi-local energy' [133]. Here, as in the BrownYork definition, $k^{0}$ and $l^{0}$ give the 'reference term' that should be fixed in a separate procedure. Note that it is $E_{\mathrm{E}}(\mathcal{S})$ that is referenced and not the mean curvatures $k$ and $l$, i.e. $E_{\mathrm{E}}(\mathcal{S})$ is not the integral of $\sqrt{\varepsilon^{2}-j_{\vdash}^{2}}$. Apart from the fact that $M_{\Sigma}$ of Equation (7) is associated with a 3-surface, Epp's invariant quasi-local energy expression appears to be analogous to $M_{\Sigma}$ rather than to $E_{\Sigma}\left[\xi^{a}\right]$ of Equation (6) or to $Q_{\mathcal{S}}[\mathbf{K}]$ of Equation (5). However, although at first sight $E_{\mathrm{E}}(\mathcal{S})$ appears to be a quasi-local mass, it turns out in special situations that it behaves as an energy expression. In the so-called 'quasi-local rest frame', i.e. in which $l=0$, it reduces to the Brown-York expression provided $k$ is positive. Note that $Q^{a}$ must be spacelike to have a quasi-local rest frame. This condition can be interpreted as some very weak convexity condition on $\mathcal{S}$. In particular, $k$ is not needed to be positive, only $k^{2}>l^{2}$ is required. While $E_{\mathrm{BY}}$ is sensitive to the sign of $k, E_{\mathrm{E}}$ is not. Hence $E_{\mathrm{E}}(\mathcal{S})$ is not simply the value of the Brown-York expression in the quasi-local rest frame.

\subsubsection{The definition of the reference configuration}

The subtraction term in Equation $(80)$ is defined through an isometric embedding of $\left(\mathcal{S}, q_{a b}\right)$ into some reference spacetime instead of a 3 -space. This spacetime is usually Minkowski or anti-deSitter spacetime. Since the 2-surface data consist of the metric, the two extrinsic curvatures and the $S O(1,1)$-gauge potential, for given $\left(\mathcal{S}, q_{a b}\right)$ and ambient spacetime $\left(M^{0}, g_{a b}^{0}\right)$ the conditions of the isometric embedding form a system of six equations for eight quantities, namely for the two extrinsic curvatures and the gauge potential $A_{e}$ (see Section 4.1.2, and especially Equations (20, 21)). Therefore, even a naive function counting argument suggests that the embedding exists, but is not unique. To have uniqueness, additional conditions must be imposed. However, since $A_{e}$ is a gauge field, one condition might be a gauge fixing in the normal bundle, and Epp's suggestion is to require that the curvature of the connection 1-form $A_{e}$ in the reference spacetime and in the physical spacetime be the same [133]. Or, in other words, not only the intrinsic metric $q_{a b}$ of $\mathcal{S}$ is required to be preserved in the embedding, but the whole curvature $f_{b c d}$ of the connection $\delta_{e}$ as well. In fact, in the connection $\delta_{e}$ on the spinor bundle $\mathbf{S}^{A}(\mathcal{S})$ both the Levi-Civita and the $S O(1,1)$ connection coefficients appear on an equal footing. (Recall that we interpreted the connection $\delta_{e}$ to be a part of the universal structure of $\mathcal{S}$.) With this choice of the reference configuration $E_{\mathrm{E}}(\mathcal{S})$ depends not only on the intrinsic 2-metric $q_{a b}$ of $\mathcal{S}$, but on the connection $\delta_{e}$ on the normal bundle as well.

Suppose that $\mathcal{S}$ is a 2 -surface in $M$ such that $k^{2}>l^{2}$ with $k>0$, and, in addition, $\left(\mathcal{S}, q_{a b}\right)$ can be embedded into the flat 3 -space with $k^{0} \geq 0$. Then there is a boost gauge (the 'quasi-local

Living Reviews in Relativity

http: //www. livingreviews.org/lrr-2004-4 
rest frame') in which $E_{\mathrm{E}}(\mathcal{S})$ coincides with the Brown-York energy $E_{\mathrm{BY}}\left(\mathcal{S}, t^{a}\right)$ in the particular boost-gauge $t^{a}$ for which $t^{a} Q_{a}=0$. Consequently, every statement stated for the latter is valid for $E_{\mathrm{E}}(\mathcal{S})$, and every example calculated for $E_{\mathrm{BY}}\left(\mathcal{S}, t^{a}\right)$ is an example for $E_{\mathrm{E}}(\mathcal{S})$ as well [133]. A clear and careful discussion of the potential alternative choices for the reference term, especially their potential connection with the angular momentum, is also given there.

\subsubsection{The various limits}

First, it should be noted that Epp's quasi-local energy is vanishing in Minkowski spacetime for any 2-surface, independently of any fleet of observers. In fact, if $\mathcal{S}$ is a 2-surface in Minkowski spacetime, then the same physical Minkowski spacetime defines the reference spacetime as well, and hence $E_{\mathrm{E}}(\mathcal{S})=0$. For round spheres in the Schwarzschild spacetime it yields the result that $E_{\mathrm{BY}}$ gave. In particular, for the horizon it is $2 m / G$ (instead of $m / G$ ), and at infinity it is $m / G$ [133]. Thus, in particular, $E_{\mathrm{E}}$ is also monotonically decreasing with $r$ in Schwarzschild spacetime.

Epp calculated the various limits of his expression too [133]. In the large sphere limit near spatial infinity he recovered the Ashtekar-Hansen form of the ADM energy, at future null infinity the Bondi-Sachs energy. The technique that is used in the latter calculations is similar to that of [93]. In non-vacuum in the small sphere limit $E_{\mathrm{E}}(\mathcal{S})$ reproduces the standard $\frac{4 \pi}{3} r^{3} T_{a b} t^{a} t^{b}$ result, but the calculations for the vacuum case are not completed. The leading term is still probably of order $r^{5}$, but its coefficient has not been calculated. Although in these calculations $t^{a}$ plays the role only of fixing the 2 -surfaces, as a result we got energy seen by the observer $t^{a}$ instead of mass. It is this reason why $E_{\mathrm{E}}(\mathcal{S})$ is considered to be energy rather than mass. In the asymptotically anti-deSitter spacetime (with the anti-de-Sitter spacetime as the reference spacetime) $E_{\mathrm{E}}$ gives zero. This motivated Epp to modify his expression to recover the mass parameter of the Schwarzschild-antide-Sitter spacetime at the infinity. The modified expression is, however, not boost-gauge invariant. Here the potential connection with the AdS/CFT correspondence is also discussed (see also [33]).

\subsection{The expression of Liu and Yau}

\subsubsection{The Liu-Yau definition}

Let $\left(\mathcal{S}, q_{a b}\right)$ be a spacelike topological 2-sphere in spacetime such that the metric has positive scalar curvature. Then by the embedding theorem there is a unique isometric embedding of $\left(\mathcal{S}, q_{a b}\right)$ into the flat 3 -space, and this embedding is unique. Let $k^{0}$ be the trace of the extrinsic curvature of $\mathcal{S}$ in this embedding, which is completely determined by $q_{a b}$ and is necessarily positive. Let $k$ and $l$ be the trace of the extrinsic curvatures of $\mathcal{S}$ in the physical spacetime corresponding to the outward pointing unit spacelike and future pointing timelike normals, respectively. Then Liu and Yau define their quasi-local energy in [253] by

$$
E_{\mathrm{LY}}(\mathcal{S}):=\frac{1}{8 \pi G} \oint_{\mathcal{S}}\left(k^{0}-\sqrt{k^{2}-l^{2}}\right) d \mathcal{S} .
$$

However, this is precisely Kijowski's 'free energy' given by Equation $(79), E_{\mathrm{LY}}(\mathcal{S})=F_{\mathrm{K}}(\mathcal{S})$, and hence we denote this by $E_{\mathrm{KLY}}(\mathcal{S})$. Obviously, this is well-defined only if, in addition to the usual convexity condition $R>0$ for the intrinsic metric, $k^{2} \geq l^{2}$ also holds, i.e. the mean curvature vector $Q_{a}$ is spacelike or null. If $k \geq 0$ then $E_{\mathrm{KLY}}(\mathcal{S}) \geq E_{\mathrm{BY}}\left(\mathcal{S}, t^{a}\right)$, where the equality holds for $t^{a}$ corresponding to the quasi-local rest frame (in the sense that it is orthogonal to the mean curvature vector of the 2-surface: $t^{a} Q_{a}=0$ ).

Isolating the gauge invariant part of the $S O(1,1)$ connection 1-form Liu and Yau defined a quasi-local angular momentum as follows [253]. Let $\alpha$ be the solution of the Poisson equation $2 q^{a b} \delta_{a} \delta_{b} \alpha=\operatorname{Im}(f)$ on $\mathcal{S}$, whose source is just the field strength of $A_{a}$ (see Equation (22)). This $\alpha$ is globally well-defined on $\mathcal{S}$ and is unique up to addition of a constant. Then define $\gamma_{a}:=A_{a}-\varepsilon_{a}{ }^{b} \delta_{b} \alpha$ 
on the domain of the connection 1-form $A_{a}$, which is easily seen to be closed. Assuming the space and time orientability of the spacetime, $A_{a}$ is globally defined on $\mathcal{S} \approx S^{2}$, and hence by $H^{1}\left(S^{2}\right)=0$ the 1-form $\gamma_{a}$ is exact: $\gamma_{a}=\delta_{a} \gamma$ for some globally defined real function $\gamma$ on $\mathcal{S}$. This function is unique up to an additive constant. Therefore, $A_{a}=\varepsilon_{a}{ }^{b} \delta_{b} \alpha+\delta_{a} \gamma$, where the first term is gauge invariant, while the second represents the gauge content of $A_{a}$. Then for any rotation Killing vector $K^{0 i}$ of the flat 3-space Liu and Yau define the quasi-local angular momentum by

$$
J_{\mathrm{LY}}\left(\mathcal{S}, K^{0 i}\right):=\frac{1}{8 \pi G} \oint_{\mathcal{S}} \varphi_{*}^{-1}\left(K^{0 i} \Pi_{i}^{0 a}\right) \varepsilon_{a}{ }^{b}\left(\delta_{b} \alpha\right) d \mathcal{S} .
$$

Here $\varphi: \mathcal{S} \rightarrow \mathbb{R}^{3}$ is the embedding and $\Pi_{i}^{0 a}$ is the projection to the tangent planes of $\varphi(\mathcal{S})$ in $\mathbb{R}^{3}$. Thus, in contrast to the Brown-York definition for the angular momentum (see Equations (68, 69, $70,71,72)$ ), in $J_{\mathrm{LY}}\left(\mathcal{S}, K^{0 i}\right)$ only the gauge invariant part $\delta_{a} \alpha$ of the gauge potential $A_{a}$ is used, and its generator vector field is the pull-back to $\mathcal{S}$ of the rotation Killing vector of the flat 3-space.

\subsubsection{The main properties of $E_{\mathrm{KLY}}(\mathcal{S})$}

The most important property of the quasi-local energy definition (81) is its positivity. Namely [253], let $\Sigma$ be a compact spacelike hypersurface with smooth boundary $\partial \Sigma$, consisting of finitely many connected components $\mathcal{S}_{1}, \ldots, \mathcal{S}_{k}$ such that each of them has positive intrinsic curvature. Suppose that the matter fields satisfy the dominant energy condition on $\Sigma$. Then $E_{\mathrm{KLY}}(\partial \Sigma):=$ $\sum_{i=1}^{k} E_{\mathrm{KLY}}\left(\mathcal{S}_{i}\right)$ is strictly positive unless the spacetime is flat along $\Sigma$. In this case $\partial \Sigma$ is necessarily connected. The proof is based on the use of Jang's equation [217], by means of which the general case can be reduced to the results of Shi and Tam in the time-symmetric case [341], stated in Section 10.1.7 (see also [399]). This positivity result is generalized in [254]: It is shown that $E_{\mathrm{KLY}}\left(\mathcal{S}_{i}\right)$ is non-negative for all $i=1, \ldots, k$, and if $E_{\mathrm{KLY}}\left(\mathcal{S}_{i}\right)=0$ for some $i$ then the spacetime is flat along $\Sigma$ and $\partial \Sigma$ is connected. (In fact, since $E_{\mathrm{KLY}}(\partial \Sigma)$ depends only on $\partial \Sigma$ but is independent of the actual $\Sigma$, if the energy condition is satisfied on the domain of dependence $D(\Sigma)$ then $E_{\mathrm{KLY}}(\partial \Sigma)=0$ implies the flatness of the spacetime along every Cauchy surface for $D(\Sigma)$, i.e. the flatness of the whole domain of dependence too.)

If $\mathcal{S}$ is an apparent horizon, i.e. $l= \pm k$, then $E_{\mathrm{KLY}}(\mathcal{S})$ is just the integral of $k^{0} /(8 \pi G)$. Then by the Minkowski inequality for the convex surfaces in the flat 3 -space (see for example [380]) one has

$$
E_{\mathrm{KLY}}(\mathcal{S})=\frac{1}{8 \pi G} \oint_{\mathcal{S}} k^{0} d \mathcal{S} \geq \frac{1}{8 \pi G} \sqrt{16 \pi \operatorname{Area}(\mathcal{S})}=2 \sqrt{\frac{\operatorname{Area}(\mathcal{S})}{16 \pi G^{2}}}
$$

i.e. it is not less than twice the irreducible mass of the horizon. For round spheres $E_{\mathrm{KLY}}(\mathcal{S})$ coincides with $E_{\mathrm{E}}(\mathcal{S})$, and hence it does not reduce to the standard round sphere expression $(27)$. In particular, for the event horizon of the Schwarzschild black hole it is $2 m / G$. Although the strict mathematical analysis is still lacking, $E_{\mathrm{KLY}}$ probably reproduces the correct large sphere limits in asymptotically flat spacetime (ADM and Bondi-Sachs energies), because the difference between the Brown-York, Epp, and Kijowski-Liu-Yau definitions disappear asymptotically.

However, $E_{\mathrm{KLY}}(\mathcal{S})$ can be positive even if $\mathcal{S}$ is in the Minkowski spacetime. In fact, for given intrinsic metric $q_{a b}$ on $\mathcal{S}$ (with positive scalar curvature) $\mathcal{S}$ can be embedded into the flat $\mathbb{R}^{3} ;$ this embedding is unique, and the trace of the extrinsic curvature $k^{0}$ is determined by $q_{a b}$. On the other hand, the isometric embedding of $\mathcal{S}$ in the Minkowski spacetime is not unique: The equations of the embedding (i.e. the Gauss, the Codazzi-Mainardi, and the Ricci equations) form a system of six equations for the six components of the two extrinsic curvatures $k_{a b}$ and $l_{a b}$ and the two components of the $S O(1,1)$ gauge potential $A_{e}$. Thus, even if we impose a gauge condition for the connection 1-form $A_{e}$, we have only six equations for the seven unknown quantities, leaving enough freedom to deform $\mathcal{S}$ (with given, fixed intrinsic metric) in the Minkowski spacetime to

Living Reviews in Relativity

http://www. livingreviews.org//rr-2004-4 
get positive Kijowski-Liu-Yau energy. Indeed, specific 2-surfaces in the Minkowski spacetime are given in [292] for which $E_{\mathrm{KLY}}(\mathcal{S})>0$. 


\section{Towards a Full Hamiltonian Approach}

The Hamilton-Jacobi method is only one possible strategy to define the quasi-local quantities in a large class of approaches, called the Hamiltonian or canonical approaches. Thus there is a considerable overlap between the various canonical methods, and hence the cutting of the material into two parts (Section 10 and Section 11) is, in some sense, artifical. In the previous Section 10 we reviewed those approaches that are based on the analysis of the action, while in the present we discuss those that are based primarily of the analysis of the Hamiltonian in the spirit of Regge and Teitelboim $[319]^{20}$.

By a full Hamiltonian analysis we mean a detailed study of the structure of the quasi-local phase space, including the constraints, the smearing fields, the symplectic structure and the Hamiltonian itself, according to the standard or some generalized Hamiltonian scenarios, in the traditional $3+1$ or in the fully Lorentz-covariant form, or even in the $2+2$ form, using the metric or triad/tetrad variables (or even the Weyl or Dirac spinors). In the literature of canonical general relativity (at least in the asymptotically flat context) there are examples for all these possibilities, and we report on the quasi-local investigations on the basis of the decomposition they use. Since the $2+2$ decomposition of the spacetime is less known, we also summarize its basic idea.

\subsection{The $3+1$ approaches}

There is a lot of literature on the canonical formulation of general relativity both in the traditional ADM and the Møller tetrad (or, recently, the closely related complex Ashtekar) variables. Thus it is quite surprising how little effort has been spent to systematically quasi-localize them. One motivation for the quasi-localization of the ADM-Regge-Teitelboim analysis came from the need for the microscopic understanding of black hole entropy [32, 31, 99]: What are the microscopic degrees of freedom behind the phenomenological notion of black hole entropy? Since the aim of the present paper is to review the construction of the quasi-local quantities in classical general relativity, we discuss only the classical 2-surface observables by means of which the 'quantum edge states' on the black hole event horizons were intended to be constructed.

\subsubsection{The 2-surface observables}

If $\Sigma$, the 3-manifold on which the ADM canonical variables $h_{a b}, \tilde{p}^{a b}$ are defined, has a boundary $\mathcal{S}:=\partial \Sigma$, then the usual vacuum constraints

$$
C\left[\nu, \nu^{a}\right]:=-\int_{\Sigma}\left\{\nu\left[\frac{1}{16 \pi G}^{3} R \sqrt{|h|}+\frac{16 \pi G}{\sqrt{|h|}}\left(\frac{1}{2}\left(\tilde{p}^{a b} h_{a b}\right)^{2}-\tilde{p}^{a b} \tilde{p}_{a b}\right)\right]+2 \nu_{a} D_{b} \tilde{p}^{a b}\right\} d^{3} x
$$

are differentiable with respect to the canonical variables only if the smearing fields $\nu$ and $\nu^{a}$ and the derivative $D_{a} \nu$ are vanishing on $\mathcal{S}^{21}$. However, as Balachandran, Chandar, and Momen [32, 31] and Carlip [99] realized, the boundary conditions for the smearing fields can be relaxed by adding appropriate boundary terms to the constraints. Namely, for any vector field $V^{a}$ and function $T$

\footnotetext{
${ }^{20}$ In fact, Kijowski's results could have been presented here, but the technique that he uses may justify their inclusion in the previous Section 10.

${ }^{21}$ Here we concentrate only on the genuine, finite boundary of $\Sigma$. The analysis is straightforward even in the presence of 'boundaries at infinity' at the asymptotic 'ends' of asymptotically flat $\Sigma$.
}

Living Reviews in Relativity

http: //www. livingreviews . org//rr-2004-4 
they define

$$
\begin{aligned}
O_{\mathrm{M}}\left[V^{a}\right] & :=\int_{\Sigma} \tilde{p}^{a b} \mathrm{七}_{\mathbf{V}} h_{a b} d^{3} x=C\left[0, V^{a}\right]-2 \oint_{\mathcal{S}} v_{a} V_{b} p^{a b} d \mathcal{S}, \\
O_{\mathrm{H}}[T] & :=-\int_{\Sigma} T\left[\frac{1}{16 \pi G}{ }^{3} R+\frac{16 \pi G}{|h|}\left(\frac{1}{2}\left(\tilde{p}^{a b} h_{a b}\right)^{2}-\tilde{p}^{a b} \tilde{p}_{a b}\right)\right] d \Sigma-\frac{1}{8 \pi G} \oint_{\mathcal{S}} T k d \mathcal{S}= \\
& =C[T, 0]-\frac{1}{8 \pi G} \oint_{\mathcal{S}} T k d \mathcal{S},
\end{aligned}
$$

where $k$ is the trace of the extrinsic curvature of $\mathcal{S}$ in $\Sigma$ and $q_{a b}$ is the induced metric on $\mathcal{S}$. Then $O_{\mathrm{M}}\left[V^{a}\right]$ is functionally differentiable if $V^{a}$ is tangent to $\mathcal{S}$, and $O_{\mathrm{H}}[T]$ is functionally differentiable if $D_{a} T$ is vanishing on $\mathcal{S}$ (and hence, in particular, $T$ is a [not necessarily zero] constant on $\mathcal{S}$ ) and $h_{a b}$ is fixed on $\mathcal{S}$. Furthermore, for any two such $V^{a}$ and $V^{\prime a}$ coinciding on $\mathcal{S}$, the difference $O_{\mathrm{M}}\left[V^{a}\right]-O_{\mathrm{M}}\left[V^{\prime a}\right]$ is just a momentum constraint, and, similarly, $O_{\mathrm{H}}[T]-O_{\mathrm{H}}\left[T^{\prime}\right]$ is a Hamiltonian constraint. Thus, on the constraint surface, $O_{\mathrm{M}}\left[V^{a}\right]$ and $O_{\mathrm{H}}[T]$ depend only on the value of $V^{a}$ and $T$ on $\mathcal{S}$. A direct calculation shows that their Poisson bracket with the constraints is a constraint, i.e. vanishing on the constraint surface. Therefore, $O_{\mathrm{M}}\left[V^{a}\right]$ and $O_{\mathrm{H}}[T]$ are well-defined 2-surface observables corresponding to the momentum and the Hamiltonian constraint, respectively. Moreover, the observables $O_{\mathrm{M}}\left[V^{a}\right]$ form an infinite-dimensional Lie algebra with respect to the Poisson bracket. In this Lie algebra the momentum constraints form an ideal, and the quotient of the algebra of the observables $O_{\mathrm{M}}\left[V^{a}\right]$ and the constraint ideal is still infinite-dimensional. (In the asymptotically flat case, cf. [47, 364].) In fact, $O_{\mathrm{M}}$ defines a homomorphism of the Lie algebra of the 2 -surface vector fields into the quotient algebra of these observables modulo constraints [32, 31, 99].

To understand the meaning of these observables, recall that any vector field $V^{a}$ on $\Sigma$ generates a diffeomorphism, which is an exact (gauge) symmetry of general relativity, and the role of the momentum constraint $C\left[0, V^{a}\right]$ is just to generate this gauge symmetry in the phase space. However, the boundary $\mathcal{S}$ breaks the diffeomorphism invariance of the system, and hence on the boundary the diffeomorphism gauge motions yield the observables $O_{\mathrm{M}}\left[V^{a}\right]$ and the gauge degrees of freedom give raise to physical degrees of freedom, making it possible to introduce the so-called edge states $[32,31,99]$. Evaluating $O_{\mathrm{M}}\left[V^{a}\right]$ and $O_{\mathrm{H}}[T]$ on the constraint surface we obtain

$$
O_{\mathrm{M}}\left[V^{a}\right]=-\frac{1}{8 \pi G} \oint_{\mathcal{S}} V^{a} A_{a} d \mathcal{S}, \quad O_{\mathrm{H}}[T]=-\frac{1}{8 \pi G} \oint_{\mathcal{S}} T k d \mathcal{S},
$$

i.e. these are just the integrals of the (unreferenced) Brown-York energy surface density weighted by the constant $T$ and momentum surface density contracted with $V^{a}$, respectively (see Equation (74)).

Analogous investigations were done by Husain and Major in [210]. Using Ashtekar's complex variables [17] they determined all the local boundary conditions for the canonical variables $A_{a}^{\mathbf{i}}$, $\tilde{E}_{\mathrm{i}}^{a}$ and for the lapse $N$, the shift $N^{a}$, and the internal gauge generator $N^{\mathrm{i}}$ on $\mathcal{S}$ that ensure the functional differentiability of the Gauss, the diffeomorphism, and the Hamiltonian constraints. Although there are several possibilities, they discussed the two most significant cases. In the first case the generators $N, N^{a}$, and $N^{\mathbf{i}}$ are vanishing on $\mathcal{S}$, whenever there are infinitely many 2surface observables both from the diffeomorphism and the Gauss constraints, but no observable from the Hamiltonian constraint. The structure of these observables is similar to that of those coming from the ADM diffeomorphism constraint above. The other case considered is when the canonical momentum $\tilde{E}_{\mathbf{i}}^{a}$ (and hence, in particular, the 3-metric) is fixed on the 2-boundary. Then the quasi-local energy could be an observable, as in the ADM analysis above.

All of the papers [32, 31, 99, 210] discuss the analogous phenomenon of how the gauge freedoms are getting to be true physical degrees of freedom in the presence of 2-surfaces on the 2-surfaces themselves in the Chern-Simons and BF theories. Weakening the boundary conditions further 
(allowing certain boundary terms in the variation of the constraints) a more general algebra of 'observables' can be obtained [101, 296]: They form the Virasoro algebra with a central charge. (In fact, Carlip's analysis in [101] is based on the so-called covariant Noether charge formalism below.) Since this algebra is well known in conformal field theories, this approach might be a basis of understanding the microscopic origin of the black hole entropy [100, 101, 102, 296, 103]. However, this quantum issue is beyond the scope of the present review.

\subsection{Approaches based on the double-null foliations}

\subsubsection{The $2+2$ decomposition}

The decomposition of the spacetime in a $2+2$ way with respect to two families of null hypersurfaces is as old as the study of gravitational radiation and the concept of the characteristic initial value problem (see for example [326, 306]). The basic idea is that we foliate an open subset $U$ of the spacetime by a 2-parameter family of (e.g. closed) spacelike 2-surfaces: If $\mathcal{S}$ is the typical 2 -surface, then this foliation is defined by a smooth embedding $\phi: \mathcal{S} \times(-\epsilon, \epsilon) \times(-\epsilon, \epsilon) \rightarrow U$ : $\left(p, \nu_{+}, \nu_{-}\right) \mapsto \phi\left(p, \nu_{+}, \nu_{-}\right)$. Then, keeping $\nu_{+}$fixed and varying $\nu_{-}$, or keeping $\nu_{-}$fixed and varying $\nu_{+}$, respectively, $\mathcal{S}_{\nu_{+}, \nu_{-}}:=\phi\left(\mathcal{S}, \nu_{+}, \nu_{-}\right)$defines two 1-parameter families of hypersurfaces $\Sigma_{\nu_{+}}$and $\Sigma_{\nu_{-}}$. Requiring one (or both) of the hypersurfaces $\Sigma_{\nu_{ \pm}}$to be null, we get a so-called null (or double-null, respectively) foliation of $U$. (In Section 4.1 .8 we required the hypersurfaces $\Sigma_{\nu_{ \pm}}$to be null only for the special value $\nu_{ \pm}=0$ of the parameters.) As is well known, because of the conjugate points, in the null or double null cases the foliation can be well-defined only locally. For fixed $\nu_{+}$and $p \in \mathcal{S}$ the prescription $\nu_{-} \mapsto \phi\left(p, \nu_{+}, \nu_{-}\right)$defines a curve through $\phi\left(p, \nu_{+}, 0\right) \in \mathcal{S}_{\nu_{+}, 0}$ in $\Sigma_{\nu_{+}}$, and hence a vector field $\xi_{+}^{a}:=\left(\partial / \partial \nu_{-}\right)^{a}$ tangent everywhere to $\Sigma_{+}$on $U$. The Lie bracket of $\xi_{+}^{a}$ and the analogously defined $\xi_{-}^{a}$ is zero. There are several inequivalent ways of introducing coordinates or rigid frame fields on $U$, which are fit naturally to the null or double null foliation $\left\{\mathcal{S}_{\nu_{+}, \nu_{-}}\right\}$, in which the (vacuum) Einstein equations and Bianchi identities take a relatively simple form [326, 152, 123, 348, 381, 180, 165, 82, 189].

Defining the 'time derivative' to be the Lie derivative, for example, along the vector field $\xi_{+}^{a}$, the Hilbert action can be rewritten according to the $2+2$ decomposition. Then the $2+2$ form of the Einstein equations can be derived from the corresponding action as the Euler-Lagrange equations provided the fact that the foliation is null is imposed only after the variation has made. (Otherwise, the variation of the action with respect to the less than ten nontrivial components of the metric would not yield all the 10 Einstein equations.) One can form the corresponding Hamiltonian, in which the null character of the foliation should appear as a constraint. Then the formal Hamilton equations are just the Einstein equations in their $2+2$ form [123, 381, 180, 189]. However, neither the boundary terms in this Hamiltonian nor the boundary conditions that could ensure its functional differentiability were considered. Therefore, this Hamiltonian can be 'correct' only up to boundary terms. Such a Hamiltonian was used by Hayward [180, 183] as the basis of his quasi-local energy expression discussed already in Section 6.3. (A similar energy expression was derived by Ikumi and Shiromizi [211], starting with the idea of the 'freely falling 2-surfaces'.)

\subsubsection{The $2+2$ quasi-localization of the Bondi-Sachs mass-loss}

As we mentioned in Section 6.1.3, this double-null foliation was used by Hayward [182] to quasilocalize the Bondi-Sachs mass-loss (and mass-gain) by using the Hawking energy. Thus we do not repeat the review of his results here.

Yoon investigated the vacuum field equations in a coordinate system based on a null $2+2$ foliation. Thus one family of hypersurfaces was (outgoing) null, e.g. $\mathcal{N}_{u}$, but the other was timelike, say $B_{v}$. The former defined a foliation of the latter in terms of the spacelike 2-surfaces $\mathcal{S}_{u, v}:=$ $\mathcal{N}_{u} \cap B_{v}$. Yoon found $[400,401]$ a certain 2-surface integral on $\mathcal{S}_{u, v}$, denoted by $\tilde{E}(u, v)$, for which

Living Reviews in Relativity

http: //www. livingreviews . org//rr-2004-4 
the difference $\tilde{E}\left(u_{2}, v\right)-\tilde{E}\left(u_{1}, v\right), u_{1}<u_{2}$, could be expressed as a flux integral on the portion of the timelike hypersurface $B_{v}$ between $\mathcal{S}_{u_{1}, v}$ and $\mathcal{S}_{u_{2}, v}$. In general this flux does not have a definite sign, but Yoon showed that asymptotically, when $B_{v}$ is 'pushed out to null infinity' (i.e. in the $v \rightarrow \infty$ limit in an asymptotically flat spacetime), it becomes negative definite. In fact, 'renormalizing' $\tilde{E}(u, v)$ by a subtraction term, $E(u, v):=\tilde{E}(u, v)-\sqrt{\operatorname{Area}\left(\mathcal{S}_{0, v}\right) /\left(16 \pi G^{2}\right)}$ tends to the Bondi energy, and the flux integral tends to the Bondi mass-loss between the cuts $u=u_{1}$ and $u=u_{2}$ [400, 401]. These investigations were extended for other integrals in [402, 403, 404], which are analogous to spatial momentum and angular momentum. However, all these integrals, including $\tilde{E}(u, v)$ above, depend not only on the geometry of the spacelike 2-surface $\mathcal{S}_{u, v}$ but on the $2+2$ foliation on an open neighbourhood of $\mathcal{S}_{u, v}$ too.

\subsection{The covariant approach}

\subsubsection{The covariant phase space methods}

The traditional ADM approach to conserved quantities and the Hamiltonian analysis of general relativity is based on the $3+1$ decomposition of fields and geometry. Although the results and the content of a theory may be covariant even if their form is not, the manifest spacetime covariance of a formalism may help to find the (spacetime covariant) observables and conserved quantities, boundary conditions, etc. easily. No a posteriori spacetime interpretation of the results is needed. Such a spacetime-covariant Hamiltonian formalism was initiated by Nester [280, 283].

His basic idea is to use (tensor or Dirac spinor valued) differential forms as the basic field variables on the spacetime manifold $M$. Thus his phase space is the collection of fields on the 4manifold $M$, endowed with the (generalized) symplectic structure of Kijowski and Tulczyjew [239]. He derives the field equations from the Lagrangian 4-form, and for a fixed spacetime vector field $K^{a}$ finds a Hamiltonian 3-form $H(\mathbf{K})_{a b c}$ whose integral on a spacelike hypersurface takes the form

$$
H[\mathbf{K}]=\frac{1}{8 \pi G} \int_{\Sigma} K^{a} G_{a b} \frac{1}{3 !} \varepsilon^{b} c d e+\oint_{\partial \Sigma} B\left(K^{a}\right)_{c d},
$$

the sum of the familiar ADM constraints and a boundary term. The Hamiltonian is determined from the requirement of the functional differentiability of $H[\mathbf{K}]$, i.e. that the variation $\delta H[\mathbf{K}]$ with respect to the canonical variables should not contain any boundary term on an asymptotically flat $\Sigma$ (see Sections 2.2.2, 3.2.1, and 3.2.2). For asymptotic translations the boundary term in the Hamiltonian gives the ADM energy-momentum 4-vector. In tetrad variables $H(\mathbf{K})_{a b c}$ is essentially Sparling's 3-form [345], and the 2-component spinor version of $B\left(K^{a}\right)_{c d}$ is essentially the NesterWitten 2-form contracted in the name index with the components of $K^{a}$ (see also Section 3.2.1).

The spirit of the first systematic investigations of the covariant phase space of the classical field theories $[122,20,146,251]$ is similar to that of Nester's. These ideas were recast into the systematic formalism by Wald and Iyer [389, 215, 216], the so-called covariant Noether charge formalism (see also $[388,251]$ ). This formalism generalizes many of the previous approaches: The Lagrangian 4-form may be any diffeomorphism invariant local expression of any finite order derivatives of the field variables. It gives a systematic prescription for the Noether currents, the symplectic structure, the Hamiltonian etc. In particular, the entropy of the stationary black holes turned out to be just a Noether charge derived from Hilbert's Lagrangian.

\subsubsection{Covariant quasi-local Hamiltonians with explicit reference configurations}

The quasi-local Hamiltonian for a large class of geometric theories, allowing torsion and nonmetricity of the connection, was investigated by Chen, Nester, and Tung [109, 107, 285] in the covariant approach of Nester above [280, 283]. Starting with a Lagrangian 4-form for a first order formulation of the theory and an arbitrary vector field $K^{a}$, they determine the general form of the 
Hamiltonian 3-form $H(\mathbf{K})_{a b c}$, including the boundary 2-form $B\left(K^{a}\right)_{c d}$. However, in the variation of the corresponding Hamiltonian there will be boundary terms in general. To cancel them, the boundary 2-form has to be modified. Introducing an explicit reference field $\phi^{0 A}$ and canonical momentum $\pi_{A}^{0}$ (which are solutions of the field equations), Chen, Nester, and Tung suggest (in the differential form notation) either of the two 4-covariant boundary 2-forms

$$
\begin{aligned}
& B_{\phi}\left(K^{a}\right):=\iota_{\mathbf{K}} \phi^{A} \wedge\left(\pi_{A}-\pi_{A}^{0}\right)-(-)^{k}\left(\phi^{A}-\phi^{0 A}\right) \wedge \iota_{\mathbf{K}} \pi_{A}^{0}, \\
& B_{\pi}\left(K^{a}\right):=\iota_{\mathbf{K}} \phi^{0 A} \wedge\left(\pi_{A}-\pi_{A}^{0}\right)-(-)^{k}\left(\phi^{A}-\phi^{0 A}\right) \wedge \iota_{\mathbf{K}} \pi_{A},
\end{aligned}
$$

where the configuration variable $\phi^{A}$ is some (tensor valued) $k$-form and $\iota_{\mathbf{K}} \phi^{a}$ is the interior product of the $k$-form $\phi_{a_{1} \ldots a_{k}}^{A}$ and the vector field $K^{a}$, i.e. in the abstract index formalism $\left(\iota_{\mathbf{K}} \phi^{A}\right)_{a_{2} \ldots a_{k}}=k K^{a} \phi_{a a_{2} \ldots a_{k}}^{A}$. Then the boundary term in the variation $\delta H[\mathbf{K}]$ of the Hamiltonian is the 2-surface integral on $\partial \Sigma$ of $\iota_{\mathbf{K}}\left(\delta \phi^{A} \wedge\left(\pi_{A}-\pi_{A}^{0}\right)\right)$ and $\iota_{\mathbf{K}}\left(-\left(\phi^{A}-\phi^{0 A}\right) \wedge \delta \pi_{A}\right)$, respectively. Therefore, the Hamiltonian is functionally differentiable with the boundary 2-form $B_{\phi}\left(K^{a}\right)$ if the configuration variable $\phi^{A}$ is fixed on $\partial \Sigma$, but $B_{\pi}\left(K^{a}\right)$ should be used if $\pi_{A}$ is fixed on $\partial \Sigma$. Thus the first boundary 2-form corresponds to a 4 -covariant Dirichlet-type, while the second to a 4-covariant Neumann-type boundary condition. Obviously, the Hamiltonian evaluated in the reference configuration $\left(\phi^{0 A}, \pi_{A}^{0}\right)$ gives zero. Chen and Nester show [107] that $B_{\phi}\left(K^{a}\right)$ and $B_{\pi}\left(K^{a}\right)$ are the only boundary 2-forms for which the resulting boundary 2-form $C\left(K^{a}\right)_{b c}$ in the variation $\delta H\left(K^{a}\right)_{b c d}$ of the Hamiltonian 3-form vanishes on $\partial \Sigma$, reflects the type of the boundary conditions (i.e. which fields are fixed on the boundary), and is built from the configuration and momentum variables 4-covariantly ('uniqueness'). A further remarkable property of $B_{\phi}\left(K^{a}\right)$ and $B_{\pi}\left(K^{a}\right)$ is that the corresponding Hamiltonian 3-form can be derived directly from appropriate Lagrangians. One possible choice for the vector field $K^{a}$ is to be a Killing vector of the reference geometry.

These general ideas were applied to general relativity in the tetrad formalism (and also in the Dirac spinor formulation of the theory [109, 105], yielding a Hamiltonian which is slightly different from Equation (87)) as well as in the usual metric formalism [105, 108]. In the latter it is the appropriate projections to $\partial \Sigma$ of $\phi^{\alpha \beta}:=\frac{1}{8 \pi G} \sqrt{|g|} g^{\alpha \beta}$ or $\pi_{\mu \beta}^{\alpha}:=\Gamma_{\mu \beta}^{\alpha}$ in some coordinate system $\left\{x^{\alpha}\right\}$ that is chosen to be fixed on $\partial \Sigma$. Then the dual of the corresponding Dirichlet and Neumann boundary 2 -forms, respectively, will be

$$
\begin{aligned}
& B_{\phi}^{a b}\left(K^{e}\right):=\delta_{d e f}^{a b c}\left(\Gamma_{g c}^{d}-\Gamma_{g c}^{0 d}\right) \phi^{g e} K^{f}+\delta_{e f}^{a b} \nabla_{c}^{0} K^{e}\left(\phi^{c f}-\phi^{0 c f}\right) \\
& B_{\pi}^{a b}\left(K^{a}\right):=\delta_{d e f}^{a b c}\left(\Gamma_{g c}^{d}-\Gamma_{g c}^{0 d}\right) \phi^{0 g e} K^{f}+\delta_{e f}^{a b} \nabla_{c} K^{e}\left(\phi^{c f}-\phi^{0 c f}\right) .
\end{aligned}
$$

The first terms are analogous to Freud's superpotential, while the second ones are analogous to Komar's superpotential. (Since the boundary 2 -form contains $\Gamma_{\mu \beta}^{\alpha}$ only in the form $\Gamma_{\mu \beta}^{\alpha}-\Gamma_{\mu \beta}^{0 \alpha}$, this is always tensorial. If $\Gamma_{\mu \beta}^{0 \alpha}$ is chosen to be vanishing, then the first term reduces to Freud's superpotential.) Because of the Komar-like term, the quasi-local quantities depend not only on the 2-surface data (both in the physical spacetime and the reference configuration), but on the normal directional derivative of $K^{a}$ as well. The connection between the present expressions and the similar previous results (pseudotensorial, tensorial, and quasi-local) is also discussed in [107, 105]. In particular, the expression based on the Dirichlet-type boundary 2-form (90) gives precisely the Katz-Bicak-Lynden-Bell superpotential [230]. In the spinor formulation of these ideas the vector field $K^{a}$ would be built from a Dirac spinor (or a pair of Weyl spinors). The main difficulty is, however, to find spinor fields representing both translational and boost-rotational displacements [110]. In the absence of a prescription for the reference configuration (even though that should be defined only on an open neighbourhood of the 2-surface) the construction is still not complete, even if the vector field $K^{a}$ is chosen to be a Killing vector of the reference spacetime.

A nice application of the covariant expression is a derivation of the first law of black hole thermodynamics [107]. The quasi-local energy expressions have been evaluated for several specific

Living Reviews in Relativity

http: //www. livingreviews . org//rr-2004-4 
2-surfaces. For round spheres in the Schwarzschild spacetime both the 4-covariant Dirichlet and Neumann boundary terms (with the Minkowski reference spacetime and $K^{a}$ as the timelike Killing vector $\left.(\partial / \partial t)^{a}\right)$ give $m / G$ at infinity, but at the horizon the former gives $2 m / G$ and the latter is infinite [107]. The Dirichlet boundary term gives at the spatial infinity in the Kerr-anti-de-Sitter solution the standard $m / G$ and $m a / G$ values for the energy and angular momentum, respectively [191]. Also, the center-of-mass is calculated both in the metric and the tetrad formulation of general relativity for the eccentric Schwarzschild solution at the spatial infinity [286, 287], and it was found that the 'Komar-like term' is needed to recover the correct, expected value. At the future null infinity of asymptotically flat spacetimes it gives the Bondi-Sachs energy-momentum and the expression of Katz [229, 233] for the angular momentum [192]. The general formulae are evaluated for the Kerr-Vaidya solution too.

\subsubsection{Covariant quasi-local Hamiltonians with general reference terms}

Anco and Tung investigated the possible boundary conditions and boundary terms in the quasilocal Hamiltonian using the covariant Noether charge formalism both of general relativity (with the Hilbert Lagrangian and tetrad variables) and of Yang-Mills-Higgs systems [7, 8]. (Some formulae of the journal versions were recently corrected in the latest arXiv-versions.) They considered the world tube of a compact spacelike hypersurface $\Sigma$ with boundary $\mathcal{S}:=\partial \Sigma$. Thus the spacetime domain they considered is the same as in the Brown-York approach: $D \approx \Sigma \times\left[t_{1}, t_{2}\right]$. Their evolution vector field $K^{a}$ is assumed to be tangent to the timelike boundary ${ }^{3} B \approx \partial \Sigma \times\left[t_{1}, t_{2}\right]$ of the domain $D$. They derived a criterion for the existence of a well-defined quasi-local Hamiltonian. Dirichlet and Neumann-type boundary conditions are imposed (i.e., in general relativity, the variations of the tetrad fields are restricted on ${ }^{3} B$ by requiring the induced metric $\gamma_{a b}$ to be fixed and the adaptation of the tetrad field to the boundary to be preserved, and the tetrad components $\Theta_{a b} E_{a}^{b}$ of the extrinsic curvature of ${ }^{3} B$ to be fixed, respectively). Then the general allowed boundary condition was shown to be just a mixed Dirichlet-Neumann boundary condition. The corresponding boundary terms of the Hamiltonian, written in the form $\oint_{\mathcal{S}} K^{a} P_{a} d \mathcal{S}$, were also determined [7]. The properties of the co-vectors $P_{a}^{\mathrm{D}}$ and $P_{a}^{\mathrm{N}}$ (called the Dirichlet and Neumann symplectic vectors, respectively) were investigated further in [8]. Their part tangential to $\mathcal{S}$ is not boost gauge invariant, and to evaluate them the boost gauge determined by the mean extrinsic curvature vector $Q^{a}$ is used (see Section 4.1.2). Both $P_{a}^{\mathrm{D}}$ and $P_{a}^{\mathrm{N}}$ are calculated for various spheres in several special spacetimes. In particular, for the round spheres of radius $r$ in the $t=$ const. hypersurface in the Reissner-Nordström solution $P_{a}^{\mathrm{D}}=\frac{2}{r}\left(1-2 m / r+e^{2} / r^{2}\right) \delta_{a}^{0}$ and $P_{a}^{\mathrm{N}}=-\left(m / r^{2}-e^{2} / r^{3}\right) \delta_{a}^{0}$, and hence the Dirichlet and Neumann 'energies' with respect to the static observer $K^{a}=(\partial / \partial t)^{a}$ are $\oint_{\mathcal{S}_{r}} K^{a} P_{a}^{\mathrm{D}} d \mathcal{S}_{r}=8 \pi r-16 \pi\left[m-e^{2} /(2 r)\right]$ and $\oint_{\mathcal{S}_{r}} K^{a} P_{a}^{\mathrm{N}} d \mathcal{S}_{r}=-4 \pi\left(m-e^{2} / r\right)$, respectively. Thus $P_{a}^{\mathrm{N}}$ does not reproduce the standard round sphere expression, while $P_{a}^{\mathrm{D}}$ gives the standard round sphere and correct ADM energies only if it is 'renormalized' by its own value in Minkowski spacetime [8].

Anco continued the investigation of the Dirichlet Hamiltonian in [6], which takes the form

$$
H[\mathbf{K}]=\frac{1}{8 \pi G} \int_{\Sigma} K^{a} G_{a b} \frac{1}{3 !} \varepsilon^{b}{ }_{c d e}-\frac{1}{8 \pi G} \oint_{\partial \Sigma} K^{a}\left(\tilde{Q}_{a}+A_{a}+B_{a}\right) d \mathcal{S} .
$$

Here the 2-surface $\partial \Sigma$ is assumed to be mean convex, whenever the boost gauge freedom in the $S O(1,1)$ gauge potential $A_{a}$ can be, and, indeed, is fixed by using the globally defined orthonormal vector basis $\left\{e_{0}^{a}, e_{1}^{a}\right\}$ in the normal bundle obtained by normalizing the mean curvature basis $\left\{\tilde{Q}_{a}, Q_{a}\right\}$. The vector field $K^{a}$ is still arbitrary, and $B_{a}$ is an arbitrary function of the metric $q_{a b}$ on the 2-boundary $\partial \Sigma$, i.e. of the boundary data. This $B^{a}$ is actually assumed to have the structure $B^{a}=e_{0}^{a} B$ for $B$ as an arbitrary function of $q_{a b}$. This Hamiltonian is functionally differentiable, gives the correct Einstein equations and, for solutions, its value e.g. with $K^{a}=e_{0}^{a}$ 
is the general expression of the quasi-local energy of Brown and York. (Compare Equation (92) with Equation (86), or rather with Equations (72, 73, 74).)

However, to rule out the dependence of this notion of quasi-local energy on the completely freely specifiable vector field $K^{a}$ (i.e. on three arbitrary functions on $\mathcal{S}$ ), Anco makes $K^{a}$ dynamical by linking it to the vector field $\tilde{Q}^{a}$. Namely, let $K^{a}:=c_{0}[\operatorname{Area}(\mathcal{S})]^{\frac{n}{2}}\left|\tilde{Q}_{e} \tilde{Q}^{e}\right|^{\frac{n-1}{2}} \tilde{Q}^{a}$, where $c_{0}$ and $n$ are constant, $\operatorname{Area}(\mathcal{S})$ is the area of $\mathcal{S}$, and extend this $K^{a}$ from $\mathcal{S}$ to $\Sigma$ in a smooth way. Then Anco proves that, keeping the 2-metric $q_{a b}$ and $K^{a}$ fixed on $\mathcal{S}$,

$$
H[\mathbf{K}]=\frac{1}{8 \pi G} \int_{\Sigma} K^{a} G_{a b} \frac{1}{3 !} \varepsilon^{b}{ }_{c d e}+\frac{c_{0}}{8 \pi G(n+1)}[\operatorname{Area}(\mathcal{S})]^{\frac{n}{2}} \oint_{\partial \Sigma}\left(B-\left|\tilde{Q}_{e} \tilde{Q}^{e}\right|^{\frac{n+1}{2}}\right) d \mathcal{S}
$$

is a correct Hamiltonian for the Einstein equations, where $B$ is an arbitrary function of $q_{a b}$. For $n=1$ with the choice $B=2^{\mathcal{S}} R$ the boundary term reduces to the Hawking energy, and for $n=0$ it is the Epp and Kijowski-Liu-Yau energies depending on the choice of $B$ (i.e. the definition of the reference term). For general $n$ choosing the reference term $B$ appropriately Anco gives a 1parameter generalization of the Hawking and the Epp-Kijowski-Liu-Yau-type quasi-local energies (called the 'mean curvature masses'). Also, he defines a family of quasi-local angular momenta. Using the positivity of the Kijowski-Liu-Yau energy $(n=0)$ it is shown that the higher power $(n>0)$ mean curvature masses are bounded from below. Although these masses seem to have the correct large sphere limit at spatial infinity, for general convex 2-surfaces in Minkowski spacetime they do not vanish.

\subsubsection{Pseudotensors and quasi-local quantities}

As we discussed briefly in Section 3.3.1, many, apparently different pseudotensors and $S O(1,3)$ gauge dependent energy-momentum density expressions can be recovered from a single differential form defined on the bundle $L(M)$ of linear frames over the spacetime manifold: The corresponding superpotentials are the pull-backs to $M$ of the various forms of the Nester-Witten 2 -from $u_{a b}^{\underline{k}}$ from $L(M)$ along the various local sections of the bundle [142, 266, 352, 353]. Thus the different pseudotensors are simply the gauge dependent manifestations of the same geometric object on the bundle $L(M)$ in the different gauges. Since, however, $u_{a b}^{k}$ is the unique extension of the Nester-Witten 2-form $u\left(\varepsilon \underline{K}, \bar{\varepsilon}^{\prime}\right)_{a b}$ on the principal bundle of normalized spin frames $\left\{\varepsilon \frac{K}{A}\right\}$ (given in Equation (12)), and the latter has been proven to be connected naturally to the gravitational energy-momentum, the pseudotensors appear to describe the same physics as the spinorial expressions, though in a slightly old fashioned form. That this is indeed the case was demonstrated clearly by Chang, Nester, and Chen [104, 108, 285], by showing an intimate connection between the covariant quasi-local Hamiltonian expressions and the pseudotensors. Writing the Hamiltonian $H[\mathbf{K}]$ in the form of the sum of the constraints and a boundary term, in a given coordinate system the integrand of this boundary term may be the superpotential of any of the pseudotensors. Then the requirement of the functional differentiability of $H[\mathbf{K}]$ gives the boundary conditions for the basic variables at $\partial \Sigma$. For example, for the Freud superpotential (for Einstein's pseudotensor) what is fixed on the boundary $\partial \Sigma$ is a certain piece of $\sqrt{|g|} g^{\alpha \beta}$.

Living Reviews in Relativity

http: //www . livingreviews . org//rr-2004-4 


\section{Constructions for Special Spacetimes}

\subsection{The Komar integral for spacetimes with Killing vectors}

Although the Komar integral (and, in general, the linkage (16) for some $\alpha$ ) does not satisfy our general requirements discussed in Section 4.3.1, and it does not always give the standard values in specific situations (see for example the 'factor-of-two anomaly' or the examples below), in the presence of a Killing vector the Komar integral, built from the Killing field, could be a very useful tool in practice. (For Killing fields the linkage $L_{\mathcal{S}}[\mathbf{K}]$ reduces to the Komar integral for any $\alpha$.) One of its most important properties is that in vacuum $L_{\mathcal{S}}[\mathbf{K}]$ depends only on the homology class of the 2-surface (see for example [387]): If $\mathcal{S}$ and $\mathcal{S}^{\prime}$ are any two 2-surfaces such that $\mathcal{S}-\mathcal{S}^{\prime}=\partial \Sigma$ for some compact 3-dimensional hypersurface $\Sigma$ on which the energy-momentum tensor of the matter fields is vanishing, then $L_{\mathcal{S}}[\mathbf{K}]=L_{\mathcal{S}^{\prime}}[\mathbf{K}]$. In particular, the Komar integral for the static Killing field in the Schwarzschild spacetime is the mass parameter $m$ of the solution for any 2-surface $\mathcal{S}$ surrounding the black hole, but it is zero if $\mathcal{S}$ does not.

On the other hand [371], the analogous integral in the Reissner-Nordström spacetime on a metric 2-sphere of radius $r$ is $m-e^{2} / r$, which deviates from the generally accepted round-sphere value $m-e^{2} /(2 r)$. Similarly, in Einstein's static universe for the spheres of radius $r$ in a $t=$ const. hypersurface $L_{\mathcal{S}}[\mathbf{K}]$ is zero instead of the round sphere result $\frac{4 \pi}{3} r^{3}[\mu+\lambda / 8 \pi G]$, where $\mu$ is the energy density of the matter and $\lambda$ is the cosmological constant.

\subsection{The effective mass of Kulkarni, Chellathurai, and Dadhich for the Kerr spacetime}

The Kulkarni-Chellathurai-Dadhich [244] effective mass for the Kerr spacetime is obtained from the Komar integral (i.e. the linkage with $\alpha=0$ ) using a hypersurface orthogonal vector field $X^{a}$ instead of the Killing vector $T^{a}$ of stationarity. The vector field $X^{a}$ is defined to be $T^{a}+\omega \Phi^{a}$, where $\Phi^{a}$ is the Killing vector of axi-symmetry and the function $\omega$ is $-g(T, \Phi) / g(\Phi, \Phi)$. This is timelike outside the horizon, it is the asymptotic time translation at infinity, and coincides with the null tangent on the event horizon. On the event horizon $r=r_{+}$it yields $M_{\mathrm{KCD}}=\sqrt{m^{2}-a^{2}}$, while in the limit $r \rightarrow \infty$ it is the mass parameter $m$ of the solution. The effective mass is computed for the Kerr-Newman spacetime in [106].

\subsection{The Katz-Lynden-Bell-Israel energy for static spacetimes}

Let $K^{a}$ be a hypersurface-orthogonal timelike Killing vector field, $\Sigma$ a spacelike hypersurface to which $K^{a}$ is orthogonal, and $K^{2}:=K_{a} K^{a}$. Let $\mathcal{S}_{\mathrm{K}}$ be the set of those points of $\Sigma$ where the length of the Killing field is the value $K$, i.e. $\mathcal{S}_{\mathrm{K}}$ are the equipotential surfaces in $\Sigma$, and let $D_{\mathrm{K}} \subset \Sigma$ be the set of those points where the magnitude of $K^{a}$ is not greater than $K$. Suppose that $D_{\mathrm{K}}$ is compact and connected. Katz, Lynden-Bell, and Israel [232] associate a quasi-local energy to the 2 -surfaces $\mathcal{S}_{\mathrm{K}}$ as follows. Suppose that the matter fields can be removed from int $D_{\mathrm{K}}$ and can be concentrated into a thin shell on $\mathcal{S}_{\mathrm{K}}$ in such a way that the space inside be flat but the geometry outside remain the same. Then, denoting the (necessarily distributional) energy-momentum tensor of the shell by $T_{s}^{a b}$ and assuming that it satisfies the weak energy condition, the total energy of the shell, $\int_{D_{\mathrm{K}}} K_{a} T_{s}^{a b} t_{b} d \Sigma$, is positive. Here $t_{a}$ is the future directed unit normal to $\Sigma$. Then, using the Einstein equations, the energy of the shell can be rewritten in terms of geometric objects on the 2-surface as

$$
E_{\mathrm{KLI}}\left(\mathcal{S}_{\mathrm{K}}\right):=\frac{1}{8 \pi G} K \oint_{\mathcal{S}_{\mathrm{K}}}[k] d \mathcal{S}_{\mathrm{K}},
$$

where $[k]$ is the jump across the 2-surface of the trace of the extrinsic curvatures of the 2surface itself in $\Sigma$. Remarkably enough, the Katz-Lynden-Bell-Israel quasi-local energy $E_{\mathrm{KLI}}$ in 
the form (94), associated with the equipotential surface $\mathcal{S}_{\mathrm{K}}$, is independent of any distributional matter field, and it can also be interpreted as follows. Let $h_{a b}$ be the metric on $\Sigma, k_{a b}$ the extrinsic curvature of $\mathcal{S}_{\mathrm{K}}$ in $\left(\Sigma, h_{a b}\right)$ and $k:=h^{a b} k_{a b}$. Then suppose that there is a flat metric $h_{a b}^{0}$ on $\Sigma$ such that the induced metric from $h_{a b}^{0}$ on $\mathcal{S}_{\mathrm{K}}$ coincides with that induced from $h_{a b}$, and $h_{a b}^{0}$ matches continuously to $h_{a b}$ on $\mathcal{S}_{\mathrm{K}}$. (Thus, in particular, the induced area element $d \mathcal{S}_{\mathrm{K}}$ determined on $\mathcal{S}_{\mathrm{K}}$ by $h_{a b}$, and $h_{a b}^{0}$ coincide.) Let the extrinsic curvature of $\mathcal{S}_{\mathrm{K}}$ in $h_{a b}^{0}$ be $_{0} k_{a b}$, and $k^{0}:=h^{a b} k_{a b}^{0}$. Then $E_{\mathrm{KLI}}\left(\mathcal{S}_{\mathrm{K}}\right)$ is the integral on $\mathcal{S}_{\mathrm{K}}$ of $K$ times the difference $k-k^{0}$. Apart from the overall factor $K$, this is essentially the Brown-York energy.

In asymptotically flat spacetimes $E_{\mathrm{KLI}}\left(\mathcal{S}_{\mathrm{K}}\right)$ tends to the ADM energy [232]. However, it does not reduce to the round-sphere energy in spherically symmetric spacetimes [277], and, in particular, gives zero for the event horizon of a Schwarzschild black hole.

Living Reviews in Relativity

http://www.livingreviews. org/lrr-2004-4 


\section{Applications in General Relativity}

In this part we give a very short review of some of the potential applications of the paradigm of quasi-locality in general relativity. This part of the review is far from being complete, and our claim here is not to discuss the problems considered in detail, but rather to give a collection of problems that are (effectively or potentially) related to quasi-local ideas, tools, notions, etc. In some of these problems the various quasi-local expressions and techniques have been used successfully, but others may provide new and promising areas of their application.

\subsection{Calculation of tidal heating}

According to astronomical observations, there is an intensive volcanism on the moon Io of Jupiter. One possible explanation of this phenomenon is that Jupiter is heating Io via the gravitational tidal forces (like the Moon, whose gravitational tidal forces raise the ocean's tides on the Earth). To check whether this could be really the case, one must be able to calculate how much energy is pumped into Io. However, gravitational energy (both in Newtonian theory and in general relativity) is only ambiguously defined (and hence cannot be localized), while the phenomena mentioned above cannot depend on the mathematics that we use to describe them. The first investigations intended to calculate the tidal work (or heating) of a compact massive body were based on the use of the various gravitational pseudotensors [318, 136]. It has been shown that although in the given (slow motion and isolated body) approximation the interaction energy between the body and its companion is ambiguous, the tidal work that the companion does on the body via the tidal forces is not. This is independent both of the gauge conditions [318] and the actual pseudotensor (Einstein, Møller, Bergmann, or Landau-Lifshitz) [136].

Recently, these calculations were repeated using quasi-local concepts by Booth and Creighton [76]. They calculated the time derivative of the Brown-York energy, given by Equations (72, 73). Assuming the form of the metric used in the pseudotensorial calculations, for the tidal work they recovered the gauge invariant expressions obtained in $[318,136]$. In these approximate calculations the precise form of the boundary conditions (or reference configurations) is not essential, because the results obtained by using different boundary conditions deviate from each other only in higher order.

\subsection{Geometric inequalities for black holes}

\subsubsection{On the Penrose inequality}

To rule out a certain class of potential counterexamples to the (weak) cosmic censorship hypothesis [303], Penrose derived an inequality that any asymptotically flat initial data set with (outermost) apparent horizon $\mathcal{S}$ must satisfy [305]: The ADM mass $m_{\mathrm{ADM}}$ of the data set cannot be less than the so-called irreducible mass of the horizon, $M:=\sqrt{\operatorname{Area}(\mathcal{S}) /\left(16 \pi G^{2}\right)}$ (see also [156], and for a recent review of the problem and the relevant literature see [90]). However, as stressed by BenDov [58], the more careful formulation of the inequality, due to Horowitz [202], is needed: Assuming that the dominant energy condition is satisfied, the ADM mass of the data set cannot be less than the irreducible mass of the 2 -surface $\mathcal{S}_{\text {min }}$, where $\mathcal{S}_{\text {min }}$ has the minimum area among the 2-surfaces enclosing the apparent horizon $\mathcal{S}$. In [58] a spherically symmetric asymptotically flat data set with future apparent horizon is given which violates the first, but not the second version of the Penrose inequality.

The inequality has been proven for the outermost future apparent horizons outside the outermost past apparent horizon in maximal data sets in spherically symmetric spacetimes [262] (see also [408, 185, 186]), for static black holes (using the Penrose mass, as we mentioned in Section 7.2.5) [374, 375], and for the perturbed Reissner-Nordström spacetimes [225] (see also [226]). 
Although the original specific potential counterexample has been shown not to violate the Penrose inequality [157], the inequality has not been proven for a general data set. (For the limitations of the proof of the Penrose inequality for the area of trapped surface and the Bondi mass at past null infinity [258], see [64].) If the inequality were true, then this would be a strengthened version of the positive mass theorem, providing a positive lower bound for the ADM mass.

On the other hand, for time-symmetric data sets the Penrose inequality has been proven, even in the presence of more than one black hole. The proof is based on the use of some quasi-local energy expression, mostly of Geroch or of Hawking. First it is shown that these expressions are monotonic along the normal vector field of a special foliation of the time-symmetric initial hypersurface (see Sections 6.1.3 and 6.2, and also [143]), and then the global existence of such a foliation between the apparent horizon and the 2-sphere at infinity is proven. The first complete proof of the latter was given by Huisken and Ilmanen [207, 208]. (Recently Bray used a conformal technique to give an alternative proof $[87,88,89]$.

A more general form of the conjecture, containing the electric charge $e$ of the black hole, was formulated by Gibbons [156]: The ADM mass is claimed not to be exceeded by $M+e^{2} /\left(4 G^{2} M\right)$. Although the weaker form of the inequality, the so-called Bogomolny inequality $m_{\mathrm{ADM}} \geq|e| / G$, has been proven (under assumptions on the matter content, see for example [160, 369, 257, 159, 274, 156]), Gibbons' inequality for the electric charge has been proven for special cases (for spherically symmetric spacetimes see for example [186]), and for time-symmetric initial data sets using Geroch's inverse mean curvature flow [218]. As a consequence of the results of [207, 208] the latter has become a complete proof. However, this inequality does not seem to work in the presence of more than one black hole: For a time-symmetric data set describing $n>1$ nearly extremal ReissnerNodström black holes, $M+e^{2} /\left(4 G^{2} M\right)$ can be greater than the ADM mass, where $16 \pi G M^{2}$ is either the area of the outermost marginally trapped surface [393], or the sum of the areas of the individual black hole horizons [124]. On the other hand, the weaker inequality derived from the cosmic censorship assumption, does not seem to be violated even in the presence of more than one black hole ${ }^{22}$.

If in the final state of gravitational collapse the black hole has not only electric charge but angular momentum as well, then the geometry is described by the Kerr-Newman solution. Expressing the mass parameter $m$ of the solution (which is just $G$ times the ADM mass) in terms of the irreducible mass $M$ of the horizon, the electric charge $q=e / \sqrt{G}$ and the angular momentum $J=m a / G$, we arrive at the more general form

$$
m_{\mathrm{ADM}} \geq \sqrt{\left(M+\frac{q^{2}}{4 G M}\right)^{2}+\left(\frac{J}{2 G M}\right)^{2}}
$$

of the Gibbons-Penrose inequality for asymptotically flat spacetimes with a single black hole. However, while $m_{\mathrm{ADM}}, M$, and $q$ are well-defined even in a general asymptotically flat data set, it is not clear what the angular momentum $J$ should be: Is it the magnitude of the angular momentum defined at spatial infinity (which is already well-defined) or the (as yet not defined) quasi-local angular momentum of the horizon? Or should $J$ be the length of the spatial angular momentum, or of the Pauli-Lubanski spin (or should the whole relativistic angular momentum contribute to $J)$ ? In the presence of more than one black hole only an inequality weaker than Equation (95) is expected to hold.

\subsubsection{On the hoop conjecture}

In connection with the formation of black holes and the weak cosmic censorship hypothesis another geometric inequality has also been formulated. This is the hoop conjecture of Thorne [367, 269],

\footnotetext{
${ }^{22}$ I am grateful to Sergio Dain for pointing out this to me.
}

Living Reviews in Relativity

http: //www. livingreviews . org//rr-2004-4 
saying that 'black holes with horizons form when and only when a mass $m$ gets compacted into a region whose circumference $C$ in every direction is $C \leq 4 \pi G m$ ' (see also [139, 391]). Mathematically, this conjecture is not precisely formulated: Neither the mass nor the notion of the circumference is well-defined. In certain situations the mass might be the ADM or the Bondi mass, but might be the integral of some locally defined 'mass density' as well [139, 35, 260, 240]. The most natural formulation of the hoop conjecture would be based on some spacelike 2-surface $\mathcal{S}$ and some reasonable notion of the quasi-local mass, and the trapped nature of the surface would be characterized by the mass and the 'circumference' of $\mathcal{S}$. In fact, for round spheres outside the outermost trapped surface and the standard round sphere definition of the quasi-local energy (26) one has $4 \pi G E=2 \pi r[1-\exp (-2 \alpha)]<2 \pi r=C$, where we used the fact that $r$ is an areal radius (see Section 4.2.1). If, however, $\mathcal{S}$ is not axi-symmetric then there is no natural definition (or, there are several inequivalent 'natural' definitions) of the circumference of $\mathcal{S}$. Interesting necessary and also sufficient conditions for the existence of averaged trapped surfaces in non-spherically symmetric cases, both in special asymptotically flat and cosmological spacetimes, are found in [260, 240]. For the investigations of the hoop conjecture in the Gibbons-Penrose spacetime of the collapsing thin matter shell see [36, 35, 379, 298], and for colliding black holes see [407].

\subsection{Quasi-local laws of black hole dynamics}

\subsubsection{Quasi-local thermodynamics of black holes}

Black holes are usually introduced in asymptotically flat spacetimes [172, 173, 175, 387], and hence it was natural to derive the formal laws of black hole mechanics/thermodynamics in the asymptotically flat context (see for example [34, 50, 51], and for a recent review see [392]). The discovery of the Hawking radiation [174] showed that the laws of black hole thermodynamics are not only analogous to the laws of thermodynamics, but black holes are genuine thermodynamical objects: The black hole temperature is a physical temperature, that is $\hbar c /(2 \pi k)$ times the surface gravity, and the entropy is a physical entropy, $k c^{3} /(4 G \hbar)$ times the area of the horizon (in the traditional units with the Boltzmann constant $k$, speed of light $c$, Newton's gravitational constant $G$, and Planck's constant $\hbar$ ) (see also [390]). Apparently, the detailed microscopic (quantum) theory of gravity is not needed to derive the black hole entropy, and it can be derived even from the general principles of a conformal field theory on the horizon of the black holes [100, 101, 102, 296, 103].

However, black holes are localized objects, thus one must be able to describe their properties and dynamics even at the quasi-local level. Nevertheless, beyond this rather theoretic claim, there are pragmatic reasons that force us to quasi-localize the laws of black hole dynamics. In particular, it is well known that the Schwarzschild black hole, fixing its temperature at infinity, has negative heat capacity. Similarly, in an asymptotically anti-de-Sitter spacetime fixing the black hole temperature via the normalization of the timelike Killing vector at infinity is not justified because there is no such physically distinguished Killing field (see [92]). These difficulties lead to the need of a quasilocal formulation of black hole thermodynamics. In [92] Brown, Creighton, and Mann investigated the thermal properties of the Schwarzschild-anti-de-Sitter black hole. They used the quasi-local approach of Brown and York to define the energy of the black hole on a spherical 2-surface $\mathcal{S}$ outside the horizon. Identifying the Brown-York energy with the internal (thermodynamical) energy and (in the $k=\hbar=c=1$ units) $1 /(4 G)$ times the area of the event horizon with the entropy, they calculated the temperature, surface pressure, and heat capacity. They found that these quantities do depend on the location of the surface $\mathcal{S}$. In particular, there is a critical value $T_{0}$ such that for temperatures $T$ greater than $T_{0}$ there are two black hole solutions, one with positive and one with negative heat capacity, but there are no Schwarzschild-anti-de-Sitter black holes with temperature $T$ less than $T_{0}$. In [121] the Brown-York analysis is extended to include dilaton and Yang-Mills fields, and the results are applied to stationary black holes to derive the first law of black hole thermodynamics. The so-called Noether charge formalism of Wald [389], and Iyer 
and Wald [215] can be interpreted as a generalization of the Brown-York approach from general relativity to any diffeomorphism invariant theory to derive quasi-local quantities [216]. However, this formalism gave a general expression for the black hole entropy as well: That is the Noether charge derived from the Hilbert Lagrangian corresponding to the null normal of the horizon, and explicitly this is still $1 /(4 G)$ times the area of the horizon. (For some recent related works see for example $[149,188])$.

There is an extensive literature of the quasi-local formulation of the black hole dynamics and relativistic thermodynamics in the spherically symmetric context (see for example [185, 187, 186, 190] and for non-spherically symmetric cases [275, 189, 74]). However, one should see clearly that while the laws of black hole thermodynamics above refer to the event horizon, which is a global concept in the spacetime, the subject of the recent quasi-local formulations is to describe the properties and the evolution of the so-called trapping horizon, which is a quasi-locally defined notion. (On the other hand, the investigations of [183, 181, 184] are based on energy and angular momentum definitions that are gauge dependent; see also Sections 4.1.8 and 6.3.)

\subsubsection{On the isolated horizons}

The idea of the isolated horizons (more precisely, the gradually more restrictive notions of the non-expanding, the weakly isolated and isolated horizons, and the special weakly isolated horizon called the rigidly rotating horizons) is to generalize the notion of Killing horizons by keeping their basic properties without the existence of any Killing vector in general. (For a recent review see [19] and references therein, especially [21, 18].) The phase space for asymptotically flat spacetimes containing an isolated horizon is based on a 3-manifold with an asymptotic end (or finitely many such ends) and an inner boundary. The boundary conditions on the inner boundary are determined by the precise definition of the isolated horizon. Then, obviously, the Hamiltonian will be the sum of the constraints and boundary terms, corresponding both to the ends and the horizon. Thus, by the appearance of the boundary term on the inner boundary makes the Hamiltonian partly quasi-local. It is shown that the condition of the Hamiltonian evolution of the states on the inner boundary along the evolution vector field is precisely the first law of black hole mechanics [21, 18].

Booth [75] applied the general idea of Brown and York to a domain $D$ whose boundary consists not only of two spacelike submanifolds $\Sigma_{1}$ and $\Sigma_{2}$ and a timelike one ${ }^{3} B$, but a further, internal boundary $\Delta$ as well, which is null. Thus he made the investigations of the isolated horizons fully quasi-local. Therefore, the topology of $\Sigma_{1}$ and $\Sigma_{2}$ is $S^{2} \times[a, b]$, and the inner (null) boundary is interpreted as (a part of) a non-expanding horizon. Then to have a well-defined variational principle on $D$, the Hilbert action had to be modified by appropriate boundary terms. However, requiring $\Delta$ to be a so-called rigidly rotating horizon, the boundary term corresponding to $\Delta$ and the allowed variations are considerably restricted. This made it possible to derive the 'first law of rigidly rotating horizon mechanics' quasi-locally, an analog of the first law of black hole mechanics. The first law for rigidly rotating horizons was also derived by Allemandi, Francaviglia, and Raiteri in the Einstein-Maxwell theory [4] using their Regge-Teitelboim-like approach [141].

Another concept is the notion of a dynamical horizon [25, 26]. This is a smooth spacelike hypersurface that can be foliated by a preferred family of marginally trapped 2 -spheres. By an appropriate definition of the energy and angular momentum balance equations for these quantities, carried by gravitational waves, are derived. Isolated horizons are the asymptotic state of dynamical horizons.

Living Reviews in Relativity

http: //www . livingreviews . org/lrr-2004-4 


\subsection{Entropy bounds}

\subsubsection{On Bekenstein's bounds for the entropy}

Having associated the entropy $S_{\mathrm{bh}}:=\left[k c^{3} /(4 G \hbar)\right] \operatorname{Area}(\mathcal{S})$ to the (spacelike cross section $\mathcal{S}$ of the) event horizon, it is natural to expect the generalized second law (GSL) of thermodynamics to hold, i.e. the sum $S_{\mathrm{m}}+S_{\mathrm{bh}}$ of the entropy of the matter and the black holes cannot decrease in any process. However, as Bekenstein pointed out, it is possible to construct thought experiments (e.g. the so-called Geroch process) in which the GSL is violated, unless a universal upper bound for the entropy-to-energy ratio for bounded systems exists [52, 53]. (For another resolution of the apparent contradiction to the GSL, based on the calculation of the buoyancy force in the thermal atmosphere of the black hole, see [385, 390].) In traditional units this upper bound is given by $S_{\mathrm{m}} / E \leq[2 \pi k /(\hbar c)] R$, where $E$ and $S_{\mathrm{m}}$ are, respectively, the total energy and entropy of the system, and $R$ is the radius of the sphere that encloses the system. It is remarkable that this inequality does not contain Newton's constant, and hence it can be expected to be applicable even for non-gravitating systems. Although this bound is violated for several model systems, for a wide class of systems in Minkowski spacetime the bound does hold [294, 295, 293, 54] (see also [81]). The Bekenstein bound has been extended for systems with electric charge by Zaslavskii [409], and for rotating systems by Hod [201] (see also [55, 166]). Although these bounds were derived for test bodies falling into black holes, interestingly enough these Bekenstein bounds hold for the black holes themselves provided the generalized Gibbons-Penrose inequality (95) holds: Identifying $E$ with $m_{\mathrm{ADM}} c^{2}$ and letting $R$ be a radius for which $4 \pi R^{2}$ is not less than the area of the event horizon of the black hole, Equation (95) can be rewritten in the traditional units as

$$
2 \pi \sqrt{(R E)^{2}-J^{2}} \geq \frac{\hbar c}{k} S_{\mathrm{bh}}+\pi q^{2} .
$$

Obviously, the Kerr-Newman solution saturates this inequality, and in the $q=0=J, J=$ 0 , and $q=0$ special cases (96) reduces to the upper bound given, respectively, by Bekenstein, Zaslavskii, and Hod. One should stress, however, that in general curved spacetimes the notion of energy, angular momentum, and radial distance appearing in Equation (96) are not yet welldefined. Perhaps it is just the quasi-local ideas that should be used to make them well-defined, and there is a deep connection between the Gibbons-Penrose inequality and the Bekenstein bound: The former is the geometric manifestation of the latter for black holes.

\subsubsection{On the holographic hypothesis}

In the literature there is another kind of upper bound for the entropy for a localized system, the so-called holographic bound. The holographic principle [366, 350, 81] says that, at the fundamental (quantum) level, one should be able to characterize the state of any physical system located in a compact spatial domain by degrees of freedom on the surface of the domain too, analogously to the holography by means of which a three dimensional image is encoded into a 2-dimensional surface. Consequently, the number of physical degrees of freedom in the domain is bounded from above by the area of the boundary of the domain instead of its volume, and the number of physical degrees of freedom on the 2-surface is not greater one-fourth of the area of the surface measured in Planckarea units $L_{\mathrm{P}}^{2}:=G \hbar / c^{3}$. This expectation is formulated in the (spacelike) holographic entropy bound [81]: Let $\Sigma$ be a compact spacelike hypersurface with boundary $\mathcal{S}$. Then the entropy $S(\Sigma)$ of the system in $\Sigma$ should satisfy $S(\Sigma) \leq k \operatorname{Area}(\mathcal{S}) /\left(4 L_{\mathrm{P}}^{2}\right)$. Formally, this bound can be obtained from the Bekenstein bound with the assumption that $2 E \leq R c^{4} / G$, i.e. that $R$ is not less than the Schwarzschild radius of $E$. Also, as with the Bekenstein bounds, this inequality can be violated in specific situations (see also [392, 81]).

On the other hand, there is another formulation of the holographic entropy bound, due to Bousso [80, 81]. Bousso's so-called covariant entropy bound is much more quasi-local than the 
previous formulations, and is based on spacelike 2-surfaces and the null hypersurfaces determined by the 2-surfaces in the spacetime. Its classical version has been proved by Flanagan, Marolf, and Wald [140]: If $\mathcal{N}$ is an everywhere non-contracting (or non-expanding) null hypersurface with spacelike cuts $\mathcal{S}_{1}$ and $\mathcal{S}_{2}$, then, assuming that the local entropy density of the matter is bounded by its energy density, the entropy flux $S_{\mathcal{N}}$ through $\mathcal{N}$ between the cuts $\mathcal{S}_{1}$ and $\mathcal{S}_{2}$ is bounded: $S_{\mathcal{N}} \leq k\left|\operatorname{Area}\left(\mathcal{S}_{2}\right)-\operatorname{Area}\left(\mathcal{S}_{1}\right)\right| /\left(4 L_{\mathrm{P}}^{2}\right)$. For a detailed discussion see [392, 81]. For still another, quasi-local formulation of the holographic principle see Section 2.2.5 and [365].

\subsection{Quasi-local radiative modes of GR}

In Section 8.2.3 we discussed the properties of the Dougan-Mason energy-momenta, and we saw that, under the conditions explained there, the energy-momentum is vanishing iff $D(\Sigma)$ is flat, and it is null iff $D(\Sigma)$ is a $p p$-wave geometry with pure radiative matter, and that these properties of the domain of dependence $D(\Sigma)$ are completely encoded into the geometry of the 2-surface $\mathcal{S}$. However, there is an important difference between these two statements: While in the former case we know the metric of $D(\Sigma)$, that is flat, in the second we know only that the geometry admits a constant null vector field, but we do not know the line element itself. Thus the question arises as whether the metric of $D(\Sigma)$ is also determined by the geometry of $\mathcal{S}$ even in the zero quasi-local mass case.

In [358] it was shown that under the condition above there is a complex valued function $\Phi$ on $\mathcal{S}$, describing the deviation of the anti-holomorphic and the holomorphic spinor dyads from each other, which plays the role of a potential for the curvature $F^{A}{ }_{B c d}$ on $\mathcal{S}$. Then, assuming that $\mathcal{S}$ is future and past convex and the matter is an N-type zero-rest-mass field, $\Phi$ and the value $\phi$ of the matter field on $\mathcal{S}$ determine the curvature of $D(\Sigma)$. Since the field equations for the metric of $D(\Sigma)$ reduce to Poisson-like equations with the curvature as the source, the metric of $D(\Sigma)$ is also determined by $\Phi$ and $\phi$ on $\mathcal{S}$. Therefore, the (purely radiative) pp-wave geometry and matter field on $D(\Sigma)$ are completely encoded in the geometry of $\mathcal{S}$ and complex functions defined on $\mathcal{S}$, respectively, in complete agreement with the holographic principle of the previous Section 13.4.

As we saw in Section 2.2.5, the radiative modes of the zero-rest-mass-fields in Minkowski spacetime, defined by their Fourier expansion, can be characterized quasi-locally on the globally hyperbolic subset $D(\Sigma)$ of the spacetime by the value of the Fourier modes on the appropriately convex spacelike 2 -surface $\mathcal{S}=\partial \Sigma$. Thus the two transversal radiative modes of them are encoded in certain fields on $\mathcal{S}$. On the other hand, because of the non-linearity of the Einstein equations it is difficult to define the radiative modes of general relativity. It could be done when the field equations become linear, i.e. near the null infinity, in the linear approximation and for $p p$-waves. In the first case the gravitational radiation is characterized on a cut $\mathcal{S}_{\infty}$ of the null infinity $\mathscr{I}^{+}$ by the $u$-derivative $\dot{\sigma}^{0}$ of the asymptotic shear of the outgoing null hypersurface $\mathcal{N}$ for which $\mathcal{S}_{\infty}=\mathcal{N} \cap \mathscr{I}^{+}$, i.e. by a complex function on $\mathcal{S}_{\infty}$. It is remarkable that it is precisely this complex function which yields the deviation of the holomorphic and anti-holomorphic spin frames at the null infinity (see for example [363]). The linear approximation of Einstein's theory is covered by the analysis of Section 2.2.5, thus those radiative modes can be characterized quasi-locally, while for the $p p$-waves the result of [358], reported above, gives such a quasi-local characterization in terms of a complex function measuring the deviation of the holomorphic and anti-holomorphic spin frames. However, the deviation of the holomorphic and anti-holomorphic structures on $\mathcal{S}$ can be defined even for generic 2-surfaces in generic spacetimes too, which might yield the possibility of introducing the radiative modes quasi-locally in general.

Living Reviews in Relativity

http://www. livingreviews . org//rr-2004-4 


\section{Summary: Achievements, Difficulties, and Open Issues}

In the previous sections we tried to give an objective review of the present state of the art. This section is, however, less positivistic: We close the present review by a critical discussion, evaluating those strategies, approaches etc. that are explicitly and unambiguously given and (at least in principle) applicable in any generic spacetime.

\subsection{On the Bartnik mass and the Hawking energy}

Although in the literature the notions mass and energy are used almost synonymously, in the present review we have made a distinction between them. By energy we meant the time component of the energy-momentum four-vector, i.e. a reference frame dependent quantity, while by mass the length of the energy-momentum, i.e. an invariant. In fact, these two have different properties: The quasi-local energy (both for the matter fields and for gravity according to the Dougan-Mason definition) is vanishing precisely for the 'ground state' of the theory (i.e. for vanishing energymomentum tensor in the domain of dependence $D(\Sigma)$ and flatness of $D(\Sigma)$, respectively, see Sections 2.2.5 and 8.2.3). In particular, for configurations describing pure radiation (purely radiative matter fields and $p p$-waves, respectively) the energy is positive. On the other hand, the vanishing of the quasi-local mass does not characterize the 'ground state', rather that is equivalent just to these purely radiative configurations.

The Bartnik mass is a natural quasi-localization of the ADM mass, and its monotonicity and positivity makes it a potentially very useful tool in proving various statements on the spacetime, because it fully characterizes the non-triviality of the finite Cauchy data by a single scalar. However, our personal opinion is that, just by its strict positivity for non-flat 3-dimensional domains, it overestimates the 'physical' quasi-local mass. In fact, if $\left(\Sigma, h_{a b}, \chi_{a b}\right)$ is a finite data set for a $p p$-wave geometry (i.e. a compact subset of the data set for a $p p$-wave metric), then it probably has an asymptotically flat extension $\left(\hat{\Sigma}, \hat{h}_{a b}, \hat{\chi}_{a b}\right)$ satisfying the dominant energy condition with bounded ADM energy and no apparent horizon between $\partial \Sigma$ and infinity. Thus while the DouganMason mass of $\partial \Sigma$ is zero, the Bartnik mass $m_{\mathrm{B}}(\Sigma)$ is strictly positive unless $\left(\Sigma, h_{a b}, \chi_{a b}\right)$ is trivial. Thus, this example shows that it is the procedure of taking the asymptotically flat extension that gives strictly positive mass. Indeed, one possible proof of the rigidity part of the positive energy theorem [24] (see also [354]) is to prove first that the vanishing of the ADM mass implies, through the Witten equation, that the spacetime admits a constant spinor field, i.e. it is a $p p$ wave spacetime, and then that the only asymptotically flat spacetime that admits a constant null vector field is the Minkowski spacetime. Therefore, it is just the global condition of the asymptotic flatness that rules out the possibility of non-trivial spacetimes with zero ADM mass. Hence it would be instructive to calculate the Bartnik mass for a compact part of a $p p$-wave data set. It might also be interesting to calculate its small surface limit to see its connection with the local fields (energy-momentum tensor and probably the Bel-Robinson tensor).

The other very useful definition is the Hawking energy (and its slightly modified version, the Geroch energy). Its advantage is its simplicity, calculability, and monotonicity for special families of 2-surfaces, and it has turned out to be a very effective tool in practice in proving for example the Penrose inequality. The small sphere limit calculation shows that it is energy rather than mass, so in principle one should be able to complete this to an energy-momentum 4-vector. One possibility is Equation $(39,40)$, but, as far as we are aware, its properties have not been investigated. Unfortunately, although the energy can be defined for 2-surfaces with nonzero genus, it is not clear how the 4-momentum could be extended for such surfaces. Although the Hawking energy is a welldefined 2-surface observable, it has not been linked to any systematic (Lagrangian or Hamiltonian) scenario. Perhaps it does not have any such interpretation, and it is simply a natural (but in general spacetimes for quite general 2-surfaces not quite viable) generalization of the standard 
round sphere expression (27). This view appears to be supported by the fact that the Hawking energy has strange properties for non-spherical surfaces, e.g. for 2-surfaces in Minkowski spacetime which are not metric spheres.

\subsection{On the Penrose mass}

Penrose's suggestion for the quasi-local mass (or, more generally, energy-momentum and angular momentum) was based on a promising and far-reaching strategy to use twistors at the fundamental level. The basic object of the construction, the so-called kinematical twistor, is intended to comprise both the energy-momentum and angular momentum, and is a well-defined quasi-local quantity on generic spacelike surfaces homeomorphic to $S^{2}$. It can be interpreted as the value of a quasi-local Hamiltonian, and the four independent 2-surface twistors play the role of the quasi-translations and quasi-rotations. The kinematical twistor was calculated for a large class of special 2-surfaces and gave acceptable results.

However, the construction is not complete. First, the construction does not work for 2-surfaces whose topology is different from $S^{2}$, and does not work even for certain topological 2-spheres for which the 2-surface twistor equation admits more than four independent solutions ('exceptional 2 -surfaces'). Second, two additional objects, the so-called infinity twistor and a Hermitian inner product on the space of 2-surface twistors, are needed to get the energy-momentum and angular momentum from the kinematical twistor and to ensure their reality. The latter is needed if we want to define the quasi-local mass as a norm of the kinematical twistor. However, no natural infinity twistor has been found, and no natural Hermitian scalar product can exist if the 2-surface cannot be embedded into a conformally flat spacetime. In addition, in the small surface calculations the quasi-local mass may be complex. If, however, we do not want to form invariants of the kinematical twistor (e.g. the mass), but we want to extract the energy-momentum and angular momentum from the kinematical twistor and we want them to be real, then only a special combination of the infinity twistor and the Hermitian scalar product, the so-called 'bar-hook combination' (see Equation (51)), would be needed.

To save the main body of the construction, the definition of the kinematical twistor was modified. Nevertheless, the mass in the modified constructions encountered an inherent ambiguity in the small surface approximation. One can still hope to find an appropriate 'bar-hook', and hence real energy-momentum and angular momentum, but invariants, such as norms, could not be formed.

\subsection{On the Dougan-Mason energy-momenta and the holomorphic/anti- holomorphic spin angular momenta}

From pragmatic points of view the Dougan-Mason energy-momenta (see Section 8.2) are certainly among the most successful definitions: The energy-positivity and the rigidity (zero energy implies flatness), and the intimate connection between the $p p$-waves and the vanishing of the masses make these definitions potentially useful quasi-local tools as the ADM and Bondi-Sachs energymomenta in the asymptotically flat context. Similar properties are proven for the quasi-local energy-momentum of the matter fields, in particular for the non-Abelian Yang-Mills fields, too. They depend only on the 2 -surface data on $\mathcal{S}$, they have a clear Lagrangian interpretation, and the spinor fields that they are based on can be considered as the spinor constituents of the quasitranslations of the 2-surface. In fact, in the Minkowski spacetime the corresponding spacetime vectors are precisely the restriction to $\mathcal{S}$ of the constant Killing vectors. These notions of energymomentum are linked completely to the geometry of $\mathcal{S}$, and are independent of any ad hoc choice for the 'fleet of observers' on it. On the other hand, the holomorphic/anti-holomorphic spinor fields determine a six real parameter family of orthonormal frame fields on $\mathcal{S}$, which can be interpreted as some distinguished class of observers. In addition, they reproduce the expected, correct limits in a

Living Reviews in Relativity

http: //www. livingreviews.org/lrr-2004-4 
number of special situations. In particular, these energy-momenta appear to have been completed by spin-angular momenta (see Section 9.2) in a natural way.

However, in spite of their successes, the Dougan-Mason energy-momenta and the spin-angular momenta based on Bramson's superpotential and the holomorphic/anti-holomorphic spinor fields have some unsatisfactory properties, too (see the lists of our expectations in Section 4.3). First, they are defined only for topological 2-spheres (but not for other topologies, e.g. for the torus $S^{1} \times S^{1}$ ), and they are not well-defined even for certain topological 2-spheres either. Such surfaces are, for example, past marginally trapped surfaces in the anti-holomorphic (and future marginally trapped surfaces in the holomorphic) case. Although the quasi-local mass associated with a marginally trapped surface $\mathcal{S}$ is expected to be its irreducible mass $\sqrt{\operatorname{Area}(\mathcal{S}) /\left(16 \pi G^{2}\right)}$, neither of the Dougan-Mason masses is well-defined for the bifurcation surfaces of the Kerr-Newman (or even Schwarzschild) black hole. Second, the role and the physical content of the holomorphicity/antiholomorphicity of the spinor fields is not clear. The use of the complex structure is justified $a$ posteriori by the nice physical properties of the constructions and the pure mathematical fact that it is only the holomorphy and anti-holomorphy operators in a large class of potentially acceptable first order linear differential operators acting on spinor fields that have a 2-dimensional kernel. Furthermore, since the holomorphic and anti-holomorphic constructions are not equivalent, we have two constructions instead of one, and it is not clear why we should prefer for example holomorphicity instead of anti-holomorphicity even at the quasi-local level.

The angular momentum based on Bramson's superpotential and the anti-holomorphic spinors together with the anti-holomorphic Dougan-Mason energy-momentum give acceptable Pauli-Lubanski spin for axi-symmetric zero-mass Cauchy developments, for small spheres, and at future null infinity, but the global angular momentum at the future null infinity is finite and well-defined only if the spatial 3-momentum part of the Bondi-Sachs 4-momentum is vanishing, i.e. only in the centreof-mass frame. (The spatial infinity limit of the spin-angular momenta has not been calculated.)

Thus the Nester-Witten 2-form appears to serve as an appropriate framework for defining the energy-momentum, and it is the two spinor fields which should probably be changed and a new choice would be needed. The holomorphic/anti-holomorphic spinor fields appears to be 'too rigid'. In fact, it is the topology of $\mathcal{S}$, namely the zero genus of $\mathcal{S}$, that restricts the solution space to two complex dimensions, instead of the local properties of the differential equations. (Thus, the situation is the same as in the twistorial construction of Penrose.) On the other hand, Bramson's superpotential is based on the idea of Bergmann and Thomson that the angular momentum of gravity is analogous to the spin. Thus the question arises as to whether this picture is correct, or the gravitational angular momentum also has an orbital part, whenever Bramson's superpotential describes only (the general form of) its spin part. The fact that our anti-holomorphic construction gives the correct, expected results for small spheres but unacceptable ones for large spheres near future null infinity in frames that are not centre-of-mass frames may indicate the lack of such an orbital term. This term could be neglected for small spheres, but certainly not for large spheres. For example, in the special quasi-local angular momentum of Bergqvist and Ludvigsen for the Kerr spacetime (see Section 9.3) it is the sum of Bramson's expression and a term that can be interpreted as the orbital angular momentum.

\subsection{On the Brown-York-type expressions}

The idea of Brown and York that the quasi-local conserved quantities should be introduced via the canonical formulation of the theory is quite natural. In fact, as we saw, one could arrive at their general formulae from different points of departure (functional differentiability of the Hamiltonian, 2-surface observables). If the a priori requirement that we should have a well-defined action principle for the trace- $\chi$-action yielded undoubtedly well behaving quasi-local expressions, then the results would a posteriori justify this basic requirement (like the holomorphicity or anti- 
holomorphicity of the spinor fields in the Dougan-Mason definitions). However, if not, then that might be considered as an unnecessarily restrictive assumption, and the question arises whether the present framework is wide enough to construct reasonable quasi-local energy-momentum and angular momentum.

Indeed, the basic requirement automatically yields the boundary condition that the 3-metric $\gamma_{a b}$ should be fixed on the boundary $\mathcal{S}$, and that the boundary term in the Hamiltonian should be built only from the surface stress tensor $\tau_{a b}$. Since the boundary conditions are given, no Legendre transformation of the canonical variables on the 2-surface is allowed (see the derivation of Kijowski's expression in Section 10.2). The use of $\tau_{a b}$ has important consequences. First, the quasi-local quantities depend not only on the geometry of the 2-surface $\mathcal{S}$, but on an arbitrarily chosen boost gauge, interpreted as a 'fleet of observers $t^{a}$ being at rest with respect to $\mathcal{S}$ ', too. This leaves a huge ambiguity in the Brown-York energy (three arbitrary functions of two variables, corresponding to the three boost parameters at each point of $\mathcal{S}$ ) unless a natural gauge choice is prescribed $^{23}$. Second, since $\tau_{a b}$ does not contain the extrinsic curvature of $\mathcal{S}$ in the direction $t^{a}$, which is a part of the 2-surface data, this extrinsic curvature is 'lost' from the point of view of the quasi-local quantities. Moreover, since $\tau_{a b}$ is a tensor only on the 3 -manifold ${ }^{3} B$, the integral of $K^{a} \tau_{a b} t^{b}$ on $\mathcal{S}$ is not sensitive to the component of $K^{a}$ normal to ${ }^{3} B$. The normal piece $v^{a} v_{b} K^{b}$ of the generator $K^{a}$ is 'lost' from the point of view of the quasi-local quantities.

The other important ingredient of the Brown-York construction is the prescription of the subtraction term. Considering the Gauss-Codazzi-Mainardi equations of the isometric embedding of the 2-surface into the flat 3-space (or rather into a spacelike hyperplane of Minkowski spacetime) only as a system of differential equations for the reference extrinsic curvature, this prescription - contrary to frequently appearing opinions - is as explicit as the condition of the holomorphicity/anti-holomorphicity of the spinor fields in the Dougan-Mason definition. (One essential, and from pragmatic points of view important, difference is that the Gauss-CodazziMainardi equations form an underdetermined elliptic system constrained by a nonlinear algebraic equation.) Similarly to the Dougan-Mason definitions, the general Brown-York formulae are valid for arbitrary spacelike 2-surfaces, but solutions to the equations defining the reference configuration exist certainly only for topological 2 -spheres with strictly positive intrinsic scalar curvature. Thus there are exceptional 2-surfaces here, too. On the other hand, the Brown-York expressions (both for the flat 3-space and the light cone references) work properly for large spheres.

At first sight, this choice for the definition of the subtraction term seems quite natural. However, we do not share this view. If the physical spacetime is the Minkowski one, then we expect that the geometry of the 2-surface in the reference Minkowski spacetime be the same as in the physical Minkowski spacetime. In particular, if $\mathcal{S}$ - in the physical Minkowski spacetime - does not lie in any spacelike hyperplane, then we think that it would be un-natural to require the embedding of $\mathcal{S}$ into a hyperplane of the reference Minkowski spacetime. Since in the two Minkowski spacetimes the extrinsic curvatures can be quite different, the quasi-local energy expressions based on this prescription of the reference term can be expected to yield a nonzero value even in flat spacetime. Indeed, there are explicit examples showing this defect. (Epp's definition is free of this difficulty, because he embeds the 2-surface into the Minkowski spacetime by preserving its 'universal structure'; see Section 4.1.4.)

Another objection against the embedding into flat 3-space is that it is not Lorentz covariant. As we discussed in Section 4.2.2, Lorentz covariance (together with the positivity requirement) was used to show that the quasi-local energy expression for small spheres in vacuum is of order $r^{5}$ with the Bel-Robinson 'energy' as the factor of proportionality. The Brown-York expression (even with the light cone reference $k^{0}=\sqrt{2^{\mathcal{S}} R}$ ) fails to give the Bel-Robinson 'energy'24.

\footnotetext{
${ }^{23}$ It could be interesting to clarify the consequences of the boost gauge choice that is based on the main extrinsic curvature vector $Q_{a}$, discussed in Section 4.1.2. This would rule out the arbitrary element of the construction.

${ }^{24}$ It might be interesting to see the small sphere expansion of the Kijowski and Kijowski-Liu-Yau expressions in
}

Living Reviews in Relativity

http: //www. livingreviews . org/lrr-2004-4 
Finally, in contrast to the Dougan-Mason definitions, the Brown-York type expressions are welldefined on marginally trapped surfaces. However, they yield just twice the expected irreducible mass, and they do not reproduce the standard round sphere expression, which, for non-trapped surfaces, comes out from all the other expressions discussed in the present section (including Kijowski's definition). It is remarkable that the derivation of the first law of black hole thermodynamics, based on the identification of the thermodynamical internal energy with the Brown-York energy, is independent of the definition of the subtraction term.

vacuum. 


\section{Acknowledgments}

I am grateful to Peter Aichelburg, Herbert Balasin, Robert Bartnik, Robert Beig, Chiang-Mei Chen, Piotr Chruściel, Sergio Dain, Jörg Frauendiener, Sean Hayward, Jacek Jezierski, Jerzy Kijowski, Stephen Lau, Lionel Mason, Niall Ó Murchadha, James Nester, Ezra Newman, Alexander Petrov, Walter Simon, George Sparling, Paul Tod, Roh Tung, and Helmuth Urbantke for their valuable comments, remarks, and stimulating questions. Special thanks to Jörg Frauendiener for continuous and fruitful discussions in the last eight years, to James Nester for the critical reading of an earlier version of the present manuscript, whose notes and remarks considerably improved its clarity, and to the two referees whose constructive criticism helped to make the present review more accurate and complete. Thanks are due to the Erwin Schrödinger Institute, Vienna, the Stefan Banach Center, Warsaw, the Universität Tübingen, the Max-Planck-Institut für Mathematik in den Naturwissenschaften, Leipzig, the National Center for Theoretical Sciences, Hsinchu, and the National Central University, Chungli, for hospitality, where parts of the present work were done and/or could be presented. This work was partially supported by the Hungarian Scientific Research Fund grant OTKA T042531.

Living Reviews in Relativity

http: //www. livingreviews.org/lrr-2004-4 


\section{References}

[1] Abbott, L.F., and Deser, S., "Stability of gravity with a cosmological constant", Nucl. Phys. $B, \mathbf{1 9 5}, 76-96,(1982) . \quad 4.2 .5$

[2] Aguirregabiria, J.M., Chamorro, A., and Virbhadra, K.S., "Energy and angular momentum of charged rotating black holes", Gen. Relativ. Gravit., 28, 1393-1400, (1996). Related online version (cited on 29 January 2004): http://arXiv.org/abs/gr-qc/9501002v3. 3

[3] Aichelburg, P.C., "Remark on the superposition principle for gravitational waves", Acta Phys. Austriaca, 34, 279-284, (1971). 4.2 .5

[4] Allemandi, G., Francaviglia, M., and Raiteri, M., "Energy in Einstein-Maxwell theory and the first law of isolated horizons via the Noether theorem", Class. Quantum Grav., 19, 26332655, (2002). Related online version (cited on 29 January 2004): http://arXiv.org/abs/gr-qc/0110104v1. 10.1.5, 13.3.2

[5] Alvarez-Gaumé, L., and Nelson, P., "Riemann surfaces and string theories", in de Wit, B., Fayet, P., and Grisaru, M., eds., Supersymmetry, Supergravity, Superstrings '86, Proceedings of the 4th Trieste Spring School, held at the ICTP, Trieste, Italy 7-15 April 1986, 419-510, (World Scientific, Singapore, 1986). 4.1 .1

[6] Anco, S.C., "Mean curvature flow in Hamiltonian general relativity, geometrical definitions of quasilocal mass-energy for spacelike two-surfaces, and their positivity", (2004). URL (cited on 21 February 2005): http: //arXiv.org/abs/math.DG/0402057v1. 11.3 .3

[7] Anco, S.C., and Tung, R.-S., "Covariant Hamiltonian boundary conditions in general relativity for spatially bounded spacetime regions", J. Math. Phys., 43, 5531-5566, (2002). Related online version (cited on 21 February 2005):

http://arXiv.org/abs/gr-qc/0109013v4. 1, 11.3.3

[8] Anco, S.C., and Tung, R.-S., "Properties of the symplectic structure of general relativity for spatially bounded spacetime regions", J. Math. Phys., 43, 3984-4019, (2002). Related online version (cited on 21 February 2005):

http://arXiv.org/abs/gr-qc/0109014v6. 1, 4.1.2, 11.3.3

[9] Anderson, J.L., Principles of Relativity Physics, (Academic Press, New York, U.S.A., 1967). 3

[10] Andersson, F., and Edgar, S.B., "Curvature-free asymmetric metric connections in KerrSchild spacetimes", J. Math. Phys., 39, 2859-2861, (1998). 8.3

[11] Arnowitt, R., Deser, S., and Misner, C.W., "Energy and the criteria for radiation in general relativity", Phys. Rev., 118, 1100-1104, (1960). 3.2.1

[12] Arnowitt, R., Deser, S., and Misner, C.W., "Coordinate invariance and energy expressions in general relativity", Phys. Rev., 122, 997-1006, (1961). 3.2 .1

[13] Arnowitt, R., Deser, S., and Misner, C.W., "Wave zone in general relativity", Phys. Rev., 121, 1556-1566, (1961). 3.2.1 
[14] Arnowitt, R., Deser, S., and Misner, C.W., "The dynamics of general relativity", in Witten, L., ed., Gravitation, an Introduction to Current Research, 227-265, (Wiley, New York, U.S.A., 1962). Related online version (cited on 21 February 2005):

http://arXiv.org/abs/gr-qc/0405109v1. 3.2.1

[15] Ashtekar, A., "Asymptotic structure of the gravitational field at spatial infinity", in Held, A., ed., General Relativity and Gravitation: One Hundred Years After the Birth of Albert Einstein, vol. 2, 37-69, (Plenum Press, New York, U.S.A., 1980). 3.2, 3.2.1, 3.2.2

[16] Ashtekar, A., "On the boundary conditions for gravitational and gauge fields at spatial infinity", in Flaherty, F.J., ed., Asymptotic Behavior of Mass and Spacetime Geometry, Proceedings of the conference, held at Oregon State University, Corvallis, Oregon, USA, October 17-21, 1983, vol. 202 of Lecture Notes in Physics, 95-109, (Springer, Berlin, Germany; New York, U.S.A., 1984). 3.2 .1

[17] Ashtekar, A., Lectures on Non-Perturbative Canonical Gravity, vol. 6 of Advanced Series in Astrophysics and Cosmology, (World Scientific, Singapore, 1991). 10.1.4, 21

[18] Ashtekar, A., Beetle, C., and Lewandowski, J., "Mechanics of rotating isolated horizons", Phys. Rev. D, 64, 044016-1-17, (2001). Related online version (cited on 29 January 2004): http://arXiv.org/abs/gr-qc/0103026v2. 13.3 .2

[19] Ashtekar, A., Beetle, C., and Lewandowski, J., "Geometry of generic isolated horizons", Class. Quantum Grav., 19, 1195-1225, (2002). Related online version (cited on 29 January 2004):

http://arXiv.org/abs/gr-qc/0111067v1. 13.3 .2

[20] Ashtekar, A., Bombelli, L., and Reula, O., "The covariant phase space of asymptotically flat gravitational fields", in Francaviglia, M., and Holm, D., eds., Mechanics, Analysis and Geometry: 200 Years after Lagrange, (North-Holland, Amsterdam, Netherlands; New York, U.S.A., 1990). 11.3.1

[21] Ashtekar, A., Fairhurst, S., and Krishnan, B., "Isolated horizons: Hamiltonian evolution and the first law", Phys. Rev. D, 62, 104025-1-29, (2000). Related online version (cited on 29 January 2004):

http://arXiv.org/abs/gr-qc/0005083v3. 13.3 .2

[22] Ashtekar, A., and Geroch, R., "Quantum theory of gravitation", Rep. Prog. Phys., 37, 12111256, (1974). 3.1.1, 4.2.5

[23] Ashtekar, A., and Hansen, R.O., "A unified treatment of null and spatial infinity in general relativity. I. Universal structure, asymptotic symmetries, and conserved quantities at spatial infinity", J. Math. Phys., 19, 1542-1566, (1978). 3.2.1, 3.2.2, 7.2.4

[24] Ashtekar, A., and Horowitz, G.T., "Energy-momentum of isolated systems cannot be null", Phys. Lett., 89A, 181-184, (1982). 8.2.3, 14.1

[25] Ashtekar, A., and Krishnan, B., "Dynamical horizons: Energy, angular momentum, fluxes and balance laws", Phys. Rev. Lett., 89, 261101-1-261101-4, (2002). Related online version (cited on 29 January 2004):

http://arXiv.org/abs/gr-qc/0207080v3. 13.3 .2

[26] Ashtekar, A., and Krishnan, B., "Dynamical horizons and their properties", Phys. Rev. D, 68, 104030-1-25, (2003). Related online version (cited on 29 January 2004): http://arXiv.org/abs/gr-qc/0308033v4. 13.3 .2 
[27] Ashtekar, A., and Magnon, A., "Asymptotically anti-de Sitter spacetimes", Class. Quantum Grav., 1, L39-L44, (1984). 4.2.5, 7.2.4

[28] Ashtekar, A., and Romano, J.D., "Spatial infinity as a boundary of spacetime", Class. Quantum Grav., 9, 1069-1100, (1992). 3.2 .1

[29] Ashtekar, A., and Streubel, M., "Symplectic geometry of radiative modes and conserved quantities at null infinity", Proc. R. Soc. London, Ser. A, 376, 585-607, (1981). 7.2.4

[30] Ashtekar, A., and Winicour, J., "Linkages and Hamiltonians at null infinity", J. Math. Phys., 23, 2410-2417, (1982). 3.2 .4

[31] Balachandran, A.P., Chandar, L., and Momen, A., "Edge States in Canonical Gravity", (1995). URL (cited on 29 January 2004):

http://arXiv.org/abs/gr-qc/9506006v2. 11.1, 21, 21, 21

[32] Balachandran, A.P., Momen, A., and Chandar, L., "Edge states in gravity and black hole physics", Nucl. Phys. B, 461, 581-596, (1996). Related online version (cited on 29 January 2004):

http://arXiv.org/abs/gr-qc/9412019v2. 11.1, 21, 21, 21

[33] Balasubramanian, V., and Kraus, P., "A stress tensor for anti-de-Sitter gravity", Commun. Math. Phys., 208, 413-428, (1999). Related online version (cited on 29 January 2004):

http://arXiv.org/abs/hep-th/9902121v5. 10.3 .3

[34] Bardeen, J.M., Carter, B., and Hawking, S.W., "The four laws of black hole mechanics", Commun. Math. Phys., 31, 161-170, (1973). 13.3 .1

[35] Barrabès, C., Gramain, A., Lesigne, E., and Letelier, P.S., "Geometric inequalities and the hoop conjecture", Class. Quantum Grav., 9, L105-L110, (1992). 13.2 .2

[36] Barrabès, C., Israel, W., and Letelier, P.S., "Analytic models of nonspherical collapse, cosmic censorship and the hoop conjecture", Phys. Lett. A, 160, 41-44, (1991). 13.2 .2

[37] Bartnik, R., "The mass of an asymptotically flat manifold", Commun. Pure Appl. Math., 39, 661-693, (1986). 3.2 .1

[38] Bartnik, R., "A new definition of quasi-local mass", in Blair, D.G., and Buckingham, M.J., eds., The Fifth Marcel Grossmann Meeting on recent developments in theoretical and experimental general relativity, gravitation and relativistic field theories, Proceedings of the meeting held at The University of Western Australia, 8-13 August 1988, 399-401, (World Scientific, Singapore; River Edge, U.S.A., 1989). 5.1.1, 9, 9

[39] Bartnik, R., "New definition of quasilocal mass", Phys. Rev. Lett., 62, 2346-2348, (1989). $5.1 .1,9,9,5.1 .2,5.1 .3$

[40] Bartnik, R., "Quasi-spherical metrics and prescribed scalar curvature", J. Differ. Geom., 37, 31-71, (1993). 5.1 .3

[41] Bartnik, R., "Mass and 3-metrics of non-negative scalar curvature", in Tatsien, L., ed., Proceedings of the International Congress of Mathematicians, Beijing, China 20-28 August 2002, vol. II, 231-240, (World Scientific, Singapore, 2002). Related online version (cited on 29 January 2004):

http://arXiv.org/abs/math.DG/0304259v1. 9, 5.1.2, 5.1.3, 5.2 
[42] Baskaran, D., Lau, S.R., and Petrov, A.N., "Center of mass integral in canonical general relativity", Ann. Phys. (N.Y.), 307, 90-131, (2003). Related online version (cited on 29 January 2004): http://arXiv.org/abs/gr-qc/0301069v2. 3.2.2, 10.1.7

[43] Baston, R.J., "The index of the 2-twistor equations", Twistor Newsletter, 1984(17), 31-32, (1984). 4.1.6, 4.1.7, 7.2.1

[44] Beig, R., "Integration of Einstein's equations near spatial infinity", Proc. R. Soc. London, Ser. A, 391, 295-304, (1984). 7

[45] Beig, R., "Time symmetric initial data and Penrose's quasi-local mass", Class. Quantum Grav., 8, L205-L209, (1991). 7.2.5

[46] Beig, R., "The classical theory of canonical general relativity", in Ehlers, J., and Friedrich, H., eds., Canonical Gravity: From Classical to Quantum, Proceedings of the 117th WE Heraeus Seminar, Bad Honnef, Germany, 13-17 September 1993, vol. 434 of Lecture Notes in Physics, 59-80, (Springer, Berlin, Germany; New York, U.S.A., 1994). 4.3 .1

[47] Beig, R., and Ó Murchadha, N., "The Poincaré group as the symmetry group of canonical general relativity", Ann. Phys. (N.Y.), 174, 463-498, (1987). 3.2.1, 3.2.2, 10.1.7, 21

[48] Beig, R., and Schmidt, B.G., "Einstein's equations near spatial infinity", Commun. Math. Phys., 87, 65-80, (1982). 3.2.1, 7, 7.2.4

[49] Beig, R., and Szabados, L.B., "On a global conformal invariant of initial data sets", Class. Quantum Grav., 14, 3091-3107, (1997). Related online version (cited on 29 January 2004): http://arXiv .org/abs/gr-qc/9706078v1. 7.2 .5

[50] Bekenstein, J.D., "Black holes and entropy", Phys. Rev. D, 7, 2333-2346, (1973). 13.3 .1

[51] Bekenstein, J.D., "Generalized second law of thermodynamics in black-hole physics", Phys. Rev. D, 9, 3292-3300, (1974). 13.3.1

[52] Bekenstein, J.D., "Universal upper bound on the entropy-to energy ratio for bounded systems", Phys. Rev. D, 23, 287-298, (1981). 13.4.1

[53] Bekenstein, J.D., "Black holes and everyday physics", Gen. Relativ. Gravit., 14, 355-359, (1982). $\quad 13.4 .1$

[54] Bekenstein, J.D., "On Page's examples challenging the entropy bound", (2000). URL (cited on 29 January 2004): http://arXiv.org/abs/gr-qc/0006003v3. 13.4.1

[55] Bekenstein, J.D., and Mayo, A.E., "Black hole polarization and new entropy bounds", Phys. Rev. D, 61, 024022-1-8, (1999). Related online version (cited on 29 January 2004): http://arXiv.org/abs/gr-qc/9903002v2. 13.4 .1

[56] Belinfante, F.J., "On the spin angular momentum of mesons", Physica, VI(9), 887-898, (1939). 2.1.2, 2

[57] Belinfante, F.J., "On the current and the density of the electric charge, the energy, the linear momentum and the angular momentum of arbitrary fields", Physica, VII, 449-474, (1940). 2.1.2, 2

Living Reviews in Relativity

http: //www. livingreviews.org/lrr-2004-4 
[58] Ben-Dov, I., "Penrose nequality and apparent horizons", Phys. Rev. D, 70, 124031-1-11, (2004). Related online version (cited on 21 February 2005):

http://arXiv.org/abs/gr-qc/0408066v1. 13.2.1

[59] Bergmann, P.G., "The general theory of relativity", in Flügge, S., ed., Handbuch der Physik. Vol. IV: Prinzipien der Elektrodynamik und Relativitätstheorie, 203-242, (Springer, Berlin, Germany; New York, U.S.A., 1962). 3, 3.1.5

[60] Bergmann, P.G., and Thomson, R., "Spin and angular momentum in general relativity", Phys. Rev., 89, 400-407, (1953). 3.2.4

[61] Bergqvist, G., "Positivity and definitions of mass", Class. Quantum Grav., 9, 1917-1922, (1992). $\quad$ 8.1.3, 8.2.3

[62] Bergqvist, G., "Quasilocal mass for event horizons", Class. Quantum Grav., 9, 1753-1766, (1992). $\quad 4.2 .5$

[63] Bergqvist, G., "Energy of small surfaces", Class. Quantum Grav., 11, 3013-3023, (1994). 6.3

[64] Bergqvist, G., "On the Penrose inequality and the role of auxiliary spinor fields", Class. Quantum Grav., 14, 2577-2583, (1997). 13.2 .1

[65] Bergqvist, G., "Vacuum momenta of small spheres", Class. Quantum Grav., 15, 1535-1538, (1998). $\quad 6.3$

[66] Bergqvist, G., and Ludvigsen, M., "Quasi-local mass near a point", Class. Quantum Grav., 4, L29-L32, (1987). 8.1.2, 8.1.3

[67] Bergqvist, G., and Ludvigsen, M., "Spinor propagation and quasilocal momentum for the Kerr solution", Class. Quantum Grav., 6, L133-L136, (1989). 8.3

[68] Bergqvist, G., and Ludvigsen, M., "Quasilocal momentum and angular momentum in Kerr spacetime", Class. Quantum Grav., 8, 697-701, (1991). 8.3, 9.3

[69] Bernstein, D.H., and Tod, K.P., "Penrose's quasi-local mass in a numerically computed space-time", Phys. Rev. D, 49, 2808-2820, (1994). 7.2.5

[70] Bizoń, P., and Malec, E., "On Witten's positive-energy proof for weakly asymptotically flat spacetimes", Class. Quantum Grav., 3, L123-L128, (1986). 3.2.1

[71] Bondi, H., "Gravitational waves in general relativity", Nature, 186, 535, (1960). 3.2.3

[72] Bondi, H., van den Burg, M.G.J., and Metzner, A.W.K., "Gravitational waves in general relativity VII. Waves from axi-symmetric isolated systems", Proc. R. Soc. London, Ser. A, $\mathbf{2 6 9}, 21-52,(1962) . \quad 3.2 .3$

[73] Booth, I., and Fairhurst, S., "Canonical phase space formulation of quasilocal general relativity", Class. Quantum Grav., 20, 4507-4531, (2003). Related online version (cited on 29 January 2004): http://arXiv.org/abs/gr-qc/0301123v2. 10.1.5

[74] Booth, I., and Fairhurst, S., "The first law for slowly evolving horizons", Phys. Rev. Lett., 92, 011102-1-4, (2004). Related online version (cited on 29 January 2004): http://arXiv.org/abs/gr-qc/0307087v2. 13.3 .1 
[75] Booth, I.S., "Metric-based Hamiltonians, null boundaries and isolated horizons", Class. Quantum Grav., 18, 4239-4264, (2001). Related online version (cited on 29 January 2004): http://arXiv.org/abs/gr-qc/0105009v2. 13.3 .2

[76] Booth, I.S., and Creighton, J.D.E., "A quasilocal calculation of tidal heating", Phys. Rev. D, 62, 067503-1-4, (2000). Related online version (cited on 29 January 2004): http://arXiv.org/abs/gr-qc/0003038v2. 10.1.7, 13.1

[77] Booth, I.S., and Mann, R.B., "Moving observers, nonorthogonal boundaries, and quasilocal energy", Phys. Rev. D, 59, 0640221-1-9, (1999). Related online version (cited on 29 January 2004): http://arXiv.org/abs/gr-qc/9810009v2. 14, 10.1.4, 10.1.5, 10.1.7, 10.1.8

[78] Booth, I.S., and Mann, R.B., "Static and infalling quasilocal energy of charged and naked black holes", Phys. Rev. D, 60, 124009-1-22, (1999). Related online version (cited on 29 January 2004): http://arXiv.org/abs/gr-qc/9907072v1. 10.1.4

[79] Borowiec, A., Ferraris, M., Francaviglia, M., and Volovich, I., "Energy-momentum complex for nonlinear gravitational Lagrangians in the first-order formalism", Gen. Relativ. Gravit., 26, 637-645, (1994). 3

[80] Bousso, R., "Holography in general space-times", J. High Energy Phys., 06, 028, (1999). Related online version (cited on 29 January 2004):

http://arXiv.org/abs/hep-th/9906022v2. 13.4 .2

[81] Bousso, R., "The holographic principle", Rev. Mod. Phys., 74, 825-874, (2002). Related online version (cited on 29 January 2004):

http://arXiv.org/abs/hep-th/0203101v2. 13.4.1, 13.4.2

[82] Brady, P.R., Droz, S., Israel, W., and Morsink, S.M., "Covariant double-null dynamics: (2+2)-splitting of the Einstein equations", Class. Quantum Grav., 13, 2211-2230, (1996). Related online version (cited on 29 January 2004): http://arXiv.org/abs/gr-qc/9510040v1. 11.2.1

[83] Bramson, B.D., "The alignment of frames of reference at null infinity for asymptotically flat Einstein-Maxwell manifolds", Proc. R. Soc. London, Ser. A, 341, 451-461, (1975). 3.2.3, $3.2 .4,4.1 .7,8$

[84] Bramson, B.D., "Relativistic angular momentum for asymptotically flat Einstein-Maxwell manifolds", Proc. R. Soc. London, Ser. A, 341, 463-490, (1975). 3.2.4, 8

[85] Bramson, B.D., "Physics in cone space", in Espositio, P., and Witten, L., eds., Asymptotic structure of spacetime, Proceedings of a Symposium on Asymptotic Structure of Space-Time (SOASST), held at the University of Cincinnati, Ohio, June 14-18, 1976, 273-359, (Plenum Press, New York, U.S.A., 1977). 3.2.4

[86] Bramson, B.D., "The invariance of spin", Proc. R. Soc. London, Ser. A, 364, 463-490, (1978). $\quad 3.2 .4,8$

[87] Bray, H.L., "Proof of the Riemannian Penrose inequality using the positive energy theorem", J. Differ. Geom., 59, 177-267, (2001). Related online version (cited on 29 January 2004): http://arXiv.org/abs/math.DG/9911173v1. 9, 5.2, 13.2.1 
[88] Bray, H.L., "Black holes and the Penrose inequality in general relativity", in Tatsien, L., ed., Proceedings of the International Congress of Mathematicians, Beijing, China 20-28 August 2002, vol. II, (World Scientific, Singapore, 2002). Related online version (cited on 29 January 2004):

http://arXiv.org/abs/math.DG/0304261v1. 13.2.1

[89] Bray, H.L., "Black holes, geometric flows, and the Penrose inequality in general relativity", Notices AMS, 49, 1372-1381, (2002). 13.2.1

[90] Bray, H.L., and Chruściel, P.T., "The Penrose Inequality", (2003). URL (cited on 29 January 2004):

http://arXiv.org/abs/gr-qc/0312047v1. 5.2, 13.2.1

[91] Brinkmann, H.W., "On Riemann spaces conformal to Euclidean space", Proc. Natl. Acad. Sci. USA, 9, 1-3, (1923). 17

[92] Brown, J.D., Creighton, J.D.E., and Mann, R., "Temperature, energy, and heat capacity of asymptotically anti-de-Sitter black holes", Phys. Rev. D, 50, 6394-6403, (1994). Related online version (cited on 29 January 2004):

http://arXiv.org/abs/gr-qc/9405007v1. 10.1.7, 13.3.1

[93] Brown, J.D., Lau, S.R., and York Jr, J.W., "Energy of isolated systems at retarded times as the null limit of quasilocal energy", Phys. Rev. D, 55, 1977-1984, (1997). Related online version (cited on 29 January 2004):

http://arXiv.org/abs/gr-qc/9609057v1. 10.1.7, 10.3.3

[94] Brown, J.D., Lau, S.R., and York Jr, J.W., "Canonical quasilocal energy and small spheres", Phys. Rev. D, 59, 064028-1-13, (1999). Related online version (cited on 29 January 2004): http://arXiv.org/abs/gr-qc/9810003. 6, 17, 10.1.7

[95] Brown, J.D., Lau, S.R., and York Jr, J.W., "Action and energy of the gravitational field", Ann. Phys. (N.Y.), 297, 175-218, (2002). Related online version (cited on 29 January 2004): http://arXiv.org/abs/gr-qc/0010024v3. 14, 10.1.4, 10.1.5, 10.1.7

[96] Brown, J.D., and York, J.M., "Quasilocal energy in general relativity", in Gotay, M.J., Marsden, J.E., and Moncrief, V.E., eds., Mathematical Aspects of Classical Field Theory, Proceedings of the AMS-IMS-SIAM Joint Summer Research Conference held July 20-26, 1991 at the University of Washington, Seattle, vol. 132 of Contemporary Mathematics, 129142, (American Mathematical Society, Providence, U.S.A., 1992). 10, 10.1.1, 10.1.2, 10.1.6, $17,10.1 .7,10.1 .8$

[97] Brown, J.D., and York Jr, J.W., "Quasilocal energy and conserved charges derived from the gravitational action", Phys. Rev. D, 47, 1407-1419, (1993). Related online version (cited on 29 January 2004):

http://arXiv.org/abs/gr-qc/9209012. 10, 10.1.1, 10.1.2, 10.1.5, 17, 10.1.7

[98] Cahill, M.E., and McVittie, G.C., "Spherical symmetry and mass-energy in general relativity I. General theory", J. Math. Phys., 11, 1382-1391, (1970). 4.2 .1

[99] Carlip, S., "Statistical Mechanics and Black Hole Entropy", (1995). URL (cited on 29 January 2004):

http://arXiv.org/abs/gr-qc/9509024v2. 11.1, 21, 21, 21 
[100] Carlip, S., "Black hole entropy from conformal field theory in any dimension", Phys. Rev. Lett., 82, 2828-2831, (1999). Related online version (cited on 29 January 2004):

http://arXiv.org/abs/hep-th/9812013v3. 21, 13.3.1

[101] Carlip, S., "Entropy from conformal field theory at Killing horizons", Class. Quantum Grav., 16, 3327-3348, (1999). Related online version (cited on 29 January 2004):

http://arXiv.org/abs/gr-qc/9906126v2. 21, 13.3 .1

[102] Carlip, S., "Black hole entropy from conformal field theory", Nucl. Phys. B (Proc. Suppl.), 88, 10-16, (2000). Related online version (cited on 29 January 2004):

http://arXiv.org/abs/gr-qc/9912118v1. 21, 13.3 .1

[103] Carlip, S., "Near-horizon conformal symmetry and black hole entropy", Phys. Rev. Lett., 88, 241301-1-241301-4, (2002). Related online version (cited on 29 January 2004):

http://arXiv.org/abs/gr-qc/0203001v1. 21, 13.3.1

[104] Chang, C.-C., Nester, J.M., and Chen, C.-M., "Pseudotensors and quasi-local energymomentum", Phys. Rev. Lett., 83, 1897-1901, (1999). Related online version (cited on 29 January 2004):

http://arXiv.org/abs/gr-qc/9809040v2. 3.1.2, 3.3.1, 11.3.4

[105] Chang, C.-C., Nester, J.M., and Chen, C.-M., "Energy-momentum quasi-localization for gravitating systems", in Liu, L., Luo, J., Li, X.-Z., and Hsu, J.-P., eds., Gravitation and Astrophysics, Proceedings of the Fourth International Workshop, held at Beijing Normal University, China, October 10-15, 1999, 163-173, (World Scientific, Singapore; River Edge, U.S.A., 2000). Related online version (cited on 29 January 2004):

http://arXiv.org/abs/gr-qc/9912058v1. 11.3.2, 11.3.2

[106] Chellathurai, V., and Dadhich, N., "Effective mass of a rotating black hole in a magnetic field", Class. Quantum Grav., 7, 361-370, (1990). 12.2

[107] Chen, C.-M., and Nester, J.M., "Quasilocal quantities for general relativity and other gravity theories", Class. Quantum Grav., 16, 1279-1304, (1999). Related online version (cited on 29 January 2004):

http://arXiv.org/abs/gr-qc/9809020v2. 11.3.2, 11.3.2, 11.3.2

[108] Chen, C.-M., and Nester, J.M., "A symplectic Hamiltonian derivation of quasi-local energymomentum for GR", Grav. Cosmol., 6, 257-270, (2000). Related online version (cited on 29 January 2004):

http://arXiv.org/abs/gr-qc/0001088v1. 3.1.3, 11.3.2, 11.3.4

[109] Chen, C.-M., Nester, J.M., and Tung, R.-S., "Quasilocal energy-momentum for geometric gravity theories", Phys. Lett. A, 203, 5-11, (1995). Related online version (cited on 29 January 2004):

http://arXiv.org/abs/gr-qc/9411048v2. 11.3.2, 11.3.2

[110] Chen, C.-M., Nester, J.M., and Tung, R.-S., "Spinor Formulations for Gravitational EnergyMomentum", (2002). URL (cited on 29 January 2004):

http://arXiv.org/abs/gr-qc/0209100v2. 11.3 .2

[111] Christodoulou, D., and Yau, S.-T., "Some remarks on the quasi-local mass", in Isenberg, J.A., ed., Mathematics and General Relativity, Proceedings of the AMS-IMS-SIAM Joint Summer Research Conference held June 22-28, 1986, vol. 71 of Contemporary Mathematics, 9-14, (American Mathematical Society, Providence, U.S.A., 1988). 4.3, 4.3.2, 4.3.2, 10

Living Reviews in Relativity

http: //www. livingreviews.org/lrr-2004-4 
[112] Chruściel, P.T., "Boundary conditions at spacelike infinity from a Hamiltonian point of view", in Bergmann, P.G., and de Sabbata, V., eds., Topological Properties and Global Structure of Space-time, Proceedings of a NATO Advanced Study Institute, held May 12-22, 1985, in Erice, Italy, vol. 138 of NATO ASI Series B, 49-59, (Plenum Press, New York, U.S.A., 1986). 3.2 .1

[113] Chruściel, P.T., "A remark on the positive-energy theorem", Class. Quantum Grav., 3, L115L121, (1986). 3.2 .1

[114] Chruściel, P.T., Jezierski, J., and Kijowski, J., Hamiltonian Field Theory in the Radiating Regime, vol. m70 of Lecture Notes in Physics, (Springer, Berlin, Germany; New York, U.S.A., 2002). $\quad 3.2 .3,3.2 .3,3.2 .4$

[115] Chruściel, P.T., Jezierski, J., and MacCallum, M.A.H., "Uniqueness of scalar field energy and gravitational energy in the radiating regime", Phys. Rev. Lett., 80, 5052-5055, (1998). Related online version (cited on 29 January 2004): http://arXiv.org/abs/gr-qc/9801073v1. 3.2 .3

[116] Chruściel, P.T., Jezierski, J., and MacCallum, M.A.H., "Uniqueness of the Trautman-Bondi mass", Phys. Rev. D, 58, 084001-1-16, (1998). Related online version (cited on 29 January 2004):

http://arXiv.org/abs/gr-qc/9803010v1. 3.2 .3

[117] Chruściel, P.T., and Nagy, G., "A Hamiltonian mass of asymptotically anti-de Sitter spacetimes", Class. Quantum Grav., 18, L61-L68, (2001). Related online version (cited on 29 January 2004):

http://arXiv.org/abs/hep-th/0011270v2. 4.2 .5

[118] Coleman, S., "Non-Abelian plane waves", Phys. Lett., 70B, 59-60, (1977). 2.2.5

[119] Corvino, J., "Scalar curvature deformation and a gluing construction for the Einstein constraint equations", Commun. Math. Phys., 214, 137-189, (2000). 5.1 .3

[120] Corvino, J., and Schoen, R.M., "On the Asymptotics for the Vacuum Einstein Constraint Equations", (2003). URL (cited on 29 January 2004):

http://arXiv.org/abs/gr-qc/0301071v1. 5.1 .3

[121] Creighton, J.D.E., and Mann, R., "Quasilocal thermodynamics of dilaton gravity coupled to gauge fields", Phys. Rev. D, 52, 4569-4587, (1995). Related online version (cited on 29 January 2004):

http://arXiv.org/abs/gr-qc/9505007v1. 13.3 .1

[122] Crnkovic, C., and Witten, E., "Covariant description of canonical formalism in geometrical theories", in Hawking, S.W., and Israel, W., eds., Three Hundred Years of Gravitation, 676684, (Cambridge University Press, Cambridge, U.K.; New York, U.S.A., 1987). 11.3 .1

[123] d' Inverno, R.A., and Smallwood, J., "Covariant 2+2 formalism of the initial-value problem in general relativity", Phys. Rev. D, 22, 1233-1247, (1980). 11.2.1

[124] Dain, S., personal communication, (September, 2003). 13.2.1

[125] Dain, S., and Moreschi, O.M., "General existence proof for rest frame systems in asymptotically flat spacetime", Class. Quantum Grav., 17, 3663-3672, (2000). Related online version (cited on 29 January 2004):

http://arXiv.org/abs/gr-qc/0203048v1. 3.2.4 
[126] Dougan, A.J., "Quasi-local mass for spheres", Class. Quantum Grav., 9, 2461-2475, (1992). 8.2 .4

[127] Dougan, A.J., and Mason, L.J., "Quasilocal mass constructions with positive energy", Phys. Rev. Lett., 67, 2119-2122, (1991). 8.2.1, 8.2.3, 8.2.4

[128] Dray, T., "Momentum flux at null infinity", Class. Quantum Grav., 2, L7-L10, (1985). 7.2 .4

[129] Dray, T., and Streubel, M., "Angular momentum at null infinity", Class. Quantum Grav., 1, 15-26, (1984). 7.2 .4

[130] Dubois-Violette, M., and Madore, J., "Conservation laws and integrability conditions for gravitational and Yang-Mills equations", Commun. Math. Phys., 108, 213-223, (1987). $3.2 .1,3.3 .1$

[131] Eardley, D.M., "Global problems in numerical relativity", in Smarr, L.L., ed., Sources of Gravitational Radiation, Proceedings of the Battelle Seattle Workshop, July 24 - August 4, 1978, 127-138, (Cambridge University Press, Cambridge, U.K.; New York, U.S.A., 1979). $4.3,4.3 .2,4.3 .2,4.3 .3,10$

[132] Eastwood, M., and Tod, K.P., "Edth - a differential operator on the sphere", Math. Proc. Camb. Phil. Soc., 92, 317-330, (1982). 4.1 .6

[133] Epp, R.J., "Angular momentum and an invariant quasilocal energy in general relativity", Phys. Rev. D, 62, 124018-1-30, (2000). Related online version (cited on 29 January 2004): http://arXiv.org/abs/gr-qc/0003035v1. 10, 17, 10.3.1, 10.3.2, 10.3.3

[134] Exton, A.R., Newman, E.T., and Penrose, R., "Conserved quantities in the Einstein-Maxwell theory", J. Math. Phys., 10, 1566-1570, (1969). 8

[135] Fatibene, L., Ferraris, M., Francaviglia, M., and Raiteri, M., "Noether charges, Brown-York quasilocal energy, and related topics", J. Math. Phys., 42, 1173-1195, (2001). Related online version (cited on 29 January 2004):

http://arXiv.org/abs/gr-qc/0003019v1. 3.1.3, 10.1.4

[136] Favata, M., "Energy localization invariance of tidal work in general relativity", Phys. Rev. D, 63, 064013-1-14, (2001). Related online version (cited on 29 January 2004):

http://arXiv.org/abs/gr-qc/0008061v1. 13.1

[137] Ferraris, M., and Francaviglia, M., "Covariant first-order Lagrangians, energy-density and superpotentials in general relativity", Gen. Relativ. Gravit., 22, 965-985, (1990). 3

[138] Ferraris, M., and Francaviglia, M., "Conservation laws in general relativity", Class. Quantum Grav., 9, S79-S95, (1992). 3

[139] Flanagan, É.É., "Hoop conjecture for black-hole horizon formation", Phys. Rev. D, 44, 24092420, (1991). 13.2.2

[140] Flanagan, É.É., Marolf, D., and Wald, R.M., "Proof of classical versions of the Bousso entropy bound and of the generalized second law", Phys. Rev. D, 62, 084035-1-11, (2000). Related online version (cited on 29 January 2004):

http://arXiv.org/abs/hep-th/9908070v4. 13.4 .2

[141] Francaviglia, M., and Raiteri, M., "Hamiltonian, energy and entropy in general relativity with non-orthogonal boundaries", Class. Quantum Grav., 19, 237-258, (2002). Related online version (cited on 29 January 2004):

http://arXiv.org/abs/gr-qc/0107074v1. 2.1.2, 10.1.5, 13.3.2

Living Reviews in Relativity

http: //www. livingreviews.org/lrr-2004-4 
[142] Frauendiener, J., "Geometric description of energy-momentum pseudotensors", Class. Quantum Grav., 6, L237-L241, (1989). 3.3.1, 11.3.4

[143] Frauendiener, J., "On an integral formula on hypersurfaces in general relativity", Class. Quantum Grav., 14, 2413-3423, (1997). Related online version (cited on 29 January 2004): http://arXiv.org/abs/gr-qc/9511036v1. 13.2 .1

[144] Frauendiener, J., "Conformal infinity", Living Rev. Relativity, 3, lrr-2000-4, (2000). URL (cited on 29 January 2004): http://www.livingreviews.org/lrr-2000-4. 3.2

[145] Frauendiener, J., "On the Penrose inequality", Phys. Rev. Lett., 87, 101101-1-101101-4, (2001). Related online version (cited on 29 January 2004): http://arXiv.org/abs/gr-qc/0105093v1. 10

[146] Frauendiener, J., and Sparling, G.A.J., "On the symplectic formalism for general relativity", Proc. R. Soc. London, 436, 141-153, (1992). 11.3 .1

[147] Frauendiener, J., and Szabados, L.B., "The kernel of the edth operators on higher-genus spacelike 2-surfaces", Class. Quantum Grav., 18, 1003-1014, (2001). Related online version (cited on 29 January 2004):

http://arXiv.org/abs/gr-qc/0010089. 4.1, 4.1.6

[148] Friedrich, H., "Gravitational fields near space-like and null infinity", J. Geom. Phys., 24, 83-163, (1998). 3.2 .1

[149] Garfinkle, D., and Mann, R., "Generalized entropy and Noether charge", Class. Quantum Grav., 17, 3317-3324, (2000). Related online version (cited on 29 January 2004): http://arXiv.org/abs/gr-qc/0004056v2. 13.3 .1

[150] Geroch, R., "Energy extraction", Ann. N.Y. Acad. Sci., 224, 108-117, (1973). 6.2.1, 6.2.2, 6.2 .2

[151] Geroch, R., "Asymptotic structure of space-time", in Esposito, F.P., and Witten, L., eds., Asymptotic Structure of Spacetime, Proceedings of a Symposium on Asymptotic Structure of Space-Time (SOASST), held at the University of Cincinnati, Ohio, June 14-18, 1976, 1-105, (Plenum Press, New York, U.S.A., 1977). 3.2, 3.2.1, 4.2 .4

[152] Geroch, R., Held, A., and Penrose, R., "A spacetime calculus based on pairs of null directions", J. Math. Phys., 14, 874-881, (1973). 4.1, 4.1.6, 11.2.1

[153] Geroch, R., and Winicour, J., "Linkages in general relativity", J. Math. Phys., 22, 803-812, (1981). $\quad 3.2 .3$

[154] Giachetta, G., and Sardanashvily, G., "Stress-Energy-Momentum Tensors in Lagrangian Field Theory. Part 1. Superpotentials", (1995). URL (cited on 29 January 2004): http://arXiv .org/abs/gr-qc/9510061v1. Preprint MPH-CAM-103-95. 3

[155] Giachetta, G., and Sardanashvily, G., "Stress-Energy-Momentum Tensors in Lagrangian Field Theory. Part 2. Gravitational Superpotential", (1995). URL (cited on 29 January 2004): http://arXiv.org/abs/gr-qc/9511040v1. Preprint MPH-CAM-108-95. 3

[156] Gibbons, G.W., "The isoperimetric and Bogomolny inequalities for black holes", in Willmore, T.J., and Hitchin, N.J., eds., Global Riemannian Geometry, 194-202, (Ellis Horwood; Halsted Press, Chichester, U.K.; New York, U.S.A., 1984). 4, 9, 13.2.1 
[157] Gibbons, G.W., "Collapsing shells and the isoperimetric inequality for black holes", Class. Quantum Grav., 14, 2905-2915, (1997). Related online version (cited on 29 January 2004): http://arXiv.org/abs/hep-th/9701049v1. 13.2.1

[158] Gibbons, G.W., and Hawking, S.W., "Action integrals and partition functions in general relativity", Phys. Rev. D, 15, 2752-2756, (1977). 10.1.6, 10.1.8

[159] Gibbons, G.W., Hawking, S.W., Horowitz, G.T., and Perry, M.J., "Positive mass theorem for black holes", Commun. Math. Phys., 88, 295-308, (1983). 3.2.1, 9, 13.2 .1

[160] Gibbons, G.W., and Hull, C.M., "A Bogomolny bound for general relativity and solutions in N=2 supergravity", Phys. Lett. B, 109, 190-194, (1982). 13.2 .1

[161] Gibbons, G.W., Hull, C.M., and Warner, N.P., "The stability of gauged supergravity", Nucl. Phys. B, 218, 173-190, (1983). 4.2.5

[162] Goldberg, J.N., "Conservation laws in general relativity", Phys. Rev., 111, 315-320, (1958). 3

[163] Goldberg, J.N., "Invariant transformations, conservation laws, and energy-momentum", in Held, A., ed., General Relativity and Gravitation: One Hundred Years After the Birth of Albert Einstein, vol. 1, 469-489, (Plenum Press, New York, U.S.A., 1980). 3, 3.2, 3.2.1, $3.2 .1,3.2 .1,3.2 .3$

[164] Goldberg, J.N., "Conserved quantities at spatial and null infinity: The Penrose potential", Phys. Rev. D, 41, 410-417, (1990). 11

[165] Goldberg, J.N., and Soteriou, C., "Canonical general relativity on a null surface with coordinate and gauge fixing", Class. Quantum Grav., 12, 2779-2797, (1995). 11.2.1

[166] Gour, G., "Entropy bounds for charged and rotating systems", Class. Quantum Grav., 20, 3403-3412, (2003). Related online version (cited on 29 January 2004):

http://arXiv.org/abs/gr-qc/0302117v1. 13.4.1

[167] Güven, R., "Solutions for gravity coupled to non-Abelian plane waves", Phys. Rev. D, 19, 471-472, (1979). $\quad 8.2 .3$

[168] Haag, R., Local Quantum Physics, Fields, Particles, Algebras, Texts and Monographs in Physics, (Springer, Berlin, Germany; New York, U.S.A., 1992). 2.2, 2.2.1, 3.3.2, 9.2

[169] Haag, R., and Kastler, D., "An algebraic approach to quantum field theory", J. Math. Phys., 5, 848-861, (1964). 2.2, 2.2.1, 3.3.2

[170] Harnett, G., "The flat generalized affine connection and twistors for the Kerr solution", Class. Quantum Grav., 10, 407-415, (1993). 8.3, 9.3

[171] Hawking, S.W., "Gravitational radiation in an expanding universe", J. Math. Phys., 9, 598604, (1968). 6.1.1, 10

[172] Hawking, S.W., "Black holes in general relativity", Commun. Math. Phys., 25, 152-166, (1972). $\quad 13.3 .1$

[173] Hawking, S.W., "The Event Horizon", in DeWitt, C., and DeWitt, B.S., eds., Black Holes, Based on lectures given at the 23rd session of the Summer School of Les Houches, 1972, 1-56, (Gordon and Breach, New York, U.S.A., 1973). 13.3.1

Living Reviews in Relativity

http: //www. livingreviews . org//rr-2004-4 
[174] Hawking, S.W., "Particle creation by black holes", Commun. Math. Phys., 43, 199-220, (1975). $\quad 13.3 .1$

[175] Hawking, S.W., and Ellis, G.F.R., The Large Scale Structure of Space-Time, Cambridge Monographs on Mathematical Physics, (Cambridge University Press, Cambridge, U.K., 1973). 2.1.1, 2.2.1, 2.2.3, 4.2.1, 8.1.2, 13.3.1

[176] Hawking, S.W., and Horowitz, G., "The gravitational Hamiltonian, action, entropy and surface terms", Class. Quantum Grav., 13, 1487-1498, (1996). Related online version (cited on 29 January 2004):

http://arXiv.org/abs/gr-qc/9501014v1. 10.1.5, 10.1.7, 10.1.8

[177] Hawking, S.W., and Hunter, C.J., "The gravitational Hamiltonian in the presence of nonorthogonal boundaries", Class. Quantum Grav., 13, 2735-2752, (1996). Related online version (cited on 29 January 2004):

http://arXiv.org/abs/gr-qc/9603050v2. 14, 10.1.5, 10.1.8

[178] Hayward, G., "Gravitational action for spacetimes with nonsmooth boundaries", Phys. Rev. $D, \mathbf{4 7}, 3275-3280,(1993) . \quad 14$

[179] Hayward, G., "Quasilocal energy conditions", Phys. Rev. D, 52, 2001-2006, (1995). Related online version (cited on 29 January 2004):

http://arXiv.org/abs/gr-qc/9403039v1. 10.1.4

[180] Hayward, S.A., "Dual-null dynamics of the Einstein field", Class. Quantum Grav., 10, 779790, (1993). $\quad 11.2 .1$

[181] Hayward, S.A., "General laws of black-hole dynamics", Phys. Rev. D, 49, 6467-6474, (1994). Related online version (cited on 29 January 2004):

http://arXiv.org/abs/gr-qc/9303006v3. 4.1.8, 13.3.1

[182] Hayward, S.A., "Quasi-localization of Bondi-Sachs energy loss", Class. Quantum Grav., 11, 3037-3048, (1994). Related online version (cited on 29 January 2004):

http://arXiv.org/abs/gr-qc/9405071v1. 4.1.3, 10, 11.2.2

[183] Hayward, S.A., "Quasilocal gravitational energy", Phys. Rev. D, 49, 831-839, (1994). Related online version (cited on 29 January 2004):

http://arXiv.org/abs/gr-qc/9303030v1. 6.3, 11.2.1, 13.3.1

[184] Hayward, S.A., "Spin coefficient form of the new laws of black hole dynamics", Class. Quantum Grav., 11, 3025-3035, (1994). Related online version (cited on 29 January 2004): http://arXiv.org/abs/gr-qc/9406033v1. 13.3 .1

[185] Hayward, S.A., "Gravitational energy in spherical symmetry", Phys. Rev. D, 53, 1938-1949, (1996). Related online version (cited on 29 January 2004):

http://arXiv.org/abs/gr-qc/9408002. 4, 13.2.1, 13.3.1

[186] Hayward, S.A., "Inequalities relating area, energy, surface gravity and charge of black holes", Phys. Rev. Lett., 81, 4557-4559, (1998). Related online version (cited on 29 January 2004): http://arXiv.org/abs/gr-qc/9807003v1. 13.2.1, 13.3.1

[187] Hayward, S.A., "Unified first law of black hole dynamics and relativistic thermodynamics", Class. Quantum Grav., 15, 3147-3162, (1998). Related online version (cited on 29 January 2004):

http://arXiv.org/abs/gr-qc/9710089v2. 13.3 .1 
[188] Hayward, S.A., "Gravitational energy as Noether charge", (2000). URL (cited on 29 January 2004):

http://arXiv.org/abs/gr-qc/0004042v1. 13.3 .1

[189] Hayward, S.A., "Gravitational-wave dynamics and black-hole dynamics: second quasispherical approximation", Class. Quantum Grav., 18, 5561-5581, (2001). Related online version (cited on 29 January 2004):

http://arXiv.org/abs/gr-qc/0102013v1. 11.2.1, 13.3.1

[190] Hayward, S.A., Mukohyama, S., and Ashworth, M.C., "Dynamic black-hole entropy", Phys. Lett. A, 256, 347-350, (1999). Related online version (cited on 29 January 2004): http://arXiv.org/abs/gr-qc/9810006v1. 13.3.1

[191] Hecht, R.D., and Nester, J.M., "A new evaluation of PGT mass and spin", Phys. Lett. A, 180, 324-331, (1993). 11.3.2

[192] Hecht, R.D., and Nester, J.M., "An evaluation of mass and spin at null infinity for the PGT and GR gravity theories", Phys. Lett. A, 217, 81-89, (1996). 11.3.2

[193] Hehl, F.W., "On the energy tensor of spinning massive matter in classical field theory and general relativity", Rep. Math. Phys., 9, 55-82, (1976). 2.1.2, 2

[194] Hehl, F.W., von der Heyde, P., Kerlick, G.D., and Nester, J.M., "General relativity with spin and torsion: Foundation and prospects", Rev. Mod. Phys., 48, 393-416, (1976). 2.1.2, 2

[195] Heinz, E., "On Weyl's embedding problems", J. Math. Mech., 11, 421-454, (1962). 17

[196] Helfer, A.D., "The angular momentum of gravitational radiation", Phys. Lett. A, 150, 342$344,(1990)$. $\quad 7.2 .4$

[197] Helfer, A.D., "Difficulties with quasi-local momentum and angular momentum", Class. Quantum Grav., 9, 1001-1008, (1992). 7.2 .3

[198] Henneaux, M., Martinez, C., Troncose, R., and Zanelli, J., "Anti-de Sitter space, asymptotics, scalar fields", Phys. Rev. D, 70, 044034-1-4, (2004). Related online version (cited on 21 February 2005):

http://arXiv.org/abs/hep-th/0404236v2. 4.2.5

[199] Hernandez, W.C., and Misner, C.W., "Observer time as a coordinate in relativistic spherical hydrodynamics", Astrophys. J., 143, 452-464, (1966). 4.2.1, 4.2.1

[200] Herzlich, M., "The positive mass theorem for black holes revisited", J. Geom. Phys., 26, 97-111, (1998). 3.2.1

[201] Hod, S., "Universal entropy bound for rotating systems", Phys. Rev. D, 61, 024018-1-4, (1999). Related online version (cited on 29 January 2004):

http://arXiv.org/abs/gr-qc/9901035v2. 13.4.1

[202] Horowitz, G.T., "The positive energy theorem and its extensions", in Flaherty, F.J., ed., Asymptotic Behavior of Mass and Spacetime Geometry, Proceedings of the conference, held at Oregon State University, Corvallis, Oregon, USA, October 17-21, 1983, vol. 202 of Lecture Notes in Physics, 1-21, (Springer, Berlin, Germany; New York, U.S.A., 1984). 3.2, 3.2.1, 13.2.1

Living Reviews in Relativity

http: //www . livingreviews . org/lrr-2004-4 
[203] Horowitz, G.T., and Perry, M.J., "Gravitational energy cannot become negative", Phys. Rev. Lett., 48, 371-374, (1982). 3.2.3

[204] Horowitz, G.T., and Schmidt, B.G., "Note on gravitational energy", Proc. R. Soc. London, Ser. A, 381, 215-224, (1982). $\quad 4.2 .2,6,6.1 .2,6.2 .1$

[205] Horowitz, G.T., and Tod, K.P., "A relation between local and total energy in general relativity", Commun. Math. Phys., 85, 429-447, (1982). 3.2.1, 3.2.1, 3.2.3

[206] Hugget, S.A., and Tod, K.P., An Introduction to Twistor Theory, vol. 4 of London Mathematical Society Student Texts, (Cambridge University Press, Cambridge, U.K.; New York, U.S.A., 1985). $\quad 4.1,4.2 .5,7$

[207] Huisken, G., and Ilmanen, T., "The Riemannian Penrose inequality", Int. Math. Res. Notices, 20, 1045-1058, (1997). Related online version (cited on 29 January 2004): http://www.math.ethz.ch/ ilmanen/papers/hpanno.ps. 6.2.2, 13.2.1

[208] Huisken, G., and Ilmanen, T., "The inverse mean curvature flow and the Riemannian Penrose inequality", J. Differ. Geom., 59, 353-437, (2001). Max-Planck-Institut für Mathematik in den Naturwissenschaften Preprint No 16, Leipzig 1998. 9, 5.1.2, 6.2.2, 13.2.1

[209] Huisken, G., and Yau, S.-T., "Definition of center of mass for isolated physical systems and unique foliations by stable spheres with constant mean curvature", Invent. Math., 124, 281-311, (1996). $\quad 3.2 .2$

[210] Husain, V., and Major, S., "Gravity and BF theory defined in bounded regions", Nucl. Phys. $B, \mathbf{5 0 0}, 381-401,(1997)$. Related online version (cited on 29 January 2004):

http://arXiv.org/abs/gr-qc/9703043v2. 21

[211] Ikumi, K., and Shiromizu, T., "Freely falling 2-surfaces and the quasi-local energy", Gen. Relativ. Gravit., 31, 73-90, (1999). Related online version (cited on 29 January 2004):

http://arXiv.org/abs/gr-qc/9704020v3. 11.2.1

[212] Isenberg, J., and Nester, J.M., "Canonical gravity", in Held, A., ed., General Relativity and Gravitation: One Hundred Years After the Birth of Albert Einstein, vol. 1, 23-97, (Plenum Press, New York, U.S.A., 1980). 2.2.2

[213] Isham, C.J., "Prima facie questions in quantum gravity", in Ehlers, J., and Friedrich, H., eds., Canonical Gravity: From Classical to Quantum, Proceedings of the 117th WE Heraeus Seminar, Bad Honnef, Germany, 13-17 September 1993, vol. 434 of Lecture Notes in Physics, 1-21, (Springer, Berlin, Germany; New York, U.S.A., 1994). 3.3.1

[214] Israel, W., and Nester, J.M., "Positivity of the Bondi gravitational mass", Phys. Lett. A, 85, 259-260, (1981). 3.2 .3

[215] Iyer, V., and Wald, R.M., "Some properties of Noether charge and a proposal for dynamical black hole entropy", Phys. Rev. D, 50, 846-864, (1994). Related online version (cited on 29 January 2004):

http://arXiv.org/abs/gr-qc/9403028v1. 2.1.2, 3.1.2, 3.1.3, 11.3.1, 13.3.1

[216] Iyer, V., and Wald, R.M., "A comparison of Noether charge and Euclidean methods for computing the entropy of stationary black holes", Phys. Rev. D, 52, 4430-4439, (1995). Related online version (cited on 29 January 2004):

http://arXiv.org/abs/gr-qc/9503052v1. 11.3.1, 13.3.1 
[217] Jang, P.S., "On the positivity of energy in general relativity", J. Math. Phys., 19, 1152-1155, (1978). Erratum: J. Math. Phys. 20217 (1979). 6.2.2, 10.4.2

[218] Jang, P.S., "Note on cosmic censorship", Phys. Rev. D, 20, 834-837, (1979). 13.2 .1

[219] Jang, P.S., and Wald, R.M., "The positive energy conjecture and the cosmic censor hypothesis", J. Math. Phys., 17, 41-44, (1977). 6.2.2, 6.2.2

[220] Jeffryes, B.P., "Two-surface twistors and conformal embedding", in Flaherty, F.J., ed., Asymptotic Behavior of Mass and Spacetime Geometry, Proceedings of the conference, held at Oregon State University, Corvallis, Oregon, USA, October 17-21, 1983, vol. 202 of Lecture Notes in Physics, 177-184, (Springer, Berlin, Germany; New York, U.S.A., 1984). 7.2.3

[221] Jeffryes, B.P., "Extra' solutions to the 2-surface twistor equations", Class. Quantum Grav., 3, L9-L12, (1986). 7.2 .1

[222] Jeffryes, B.P., "The Newtonian limit of Penrose's quasi-local mass", Class. Quantum Grav., 3, 841-852, (1986). 7.2 .4

[223] Jeffryes, B.P., "2-Surface twistors, embeddings and symmetries", Proc. R. Soc. London, Ser. A, 411, 59-83, (1987). 7.2.3, 7.2.5

[224] Jezierski, J., "Positivity of mass for spacetimes with horizons", Class. Quantum Grav., 6, 1535-1539, (1989). 3.2.1

[225] Jezierski, J., "Perturbation of initial data for spherically symmetric charged black hole and Penrose conjecture", Acta Phys. Pol. B, 25, 1413-1417, (1994). 13.2 .1

[226] Jezierski, J., "Stability of Reissner-Nordström solution with respect to small perturbations of initial data", Class. Quantum Grav., 11, 1055-1068, (1994). 13.2.1

[227] Jezierski, J., and Kijowski, J., "The localization of energy in gauge field theories and in linear gravitation", Gen. Relativ. Gravit., 22, 1283-1307, (1990). 4.2 .5

[228] Julia, B., and Silva, S., "Currents and superpotentials in classical gauge invariant theories I. Local results with applications to perfect fluids and general relativity", Class. Quantum Grav., 15, 2173-2215, (1998). Related online version (cited on 29 January 2004): http://arXiv.org/abs/gr-qc/9804029v2. 2.1.2, 3

[229] Katz, J., "A note on Komar's anomalous factor", Class. Quantum Grav., 2, 423-425, (1985). $3.1 .3,3.2 .2,11.3 .2$

[230] Katz, J., Bicak, J., and Lynden-Bell, D., "Relativistic conservation laws and integral constraints for large cosmological perturbations", Phys. Rev. D, 55, 5957-5969, (1997). 3.1.3, $10.1 .4,11.3 .2$

[231] Katz, J., and Lerer, D., "On global conservation laws at null infinity", Class. Quantum Grav., 14, 2249-66, (1997). Related online version (cited on 29 January 2004): http://arXiv.org/abs/gr-qc/9612025. 3.1.2, 3.1.3

[232] Katz, J., Lynden-Bell, D., and Israel, W., "Quasilocal energy in static gravitational fields", Class. Quantum Grav., 5, 971-987, (1988). 12.3, 12.3

[233] Katz, J., and Ori, A., "Localisation of field energy", Class. Quantum Grav., 7, 787-802, (1990). 3.1.3, 11.3.2

Living Reviews in Relativity

http: //www . livingreviews . org/lrr-2004-4 
[234] Kelly, R.M., "Asymptotically anti de Sitter space-times", Twistor Newsletter, 1985(20), 11-23, (1985). 7.2 .4

[235] Kelly, R.M., Tod, K.P., and Woodhouse, N.M.J., "Quasi-local mass for small surfaces", Class. Quantum Grav., 3, 1151-1167, (1986). 4.2.2, 6, 6, 7.2.6, 7.2.6, 7.3.1, 7.3.1

[236] Kibble, T.W.B., "Lorentz invariance and the gravitational field", J. Math. Phys., 2, 212-221, (1961). $\quad 3.1 .4$

[237] Kijowski, J., "A simple derivation of canonical structure and quasi-local Hamiltonians in general relativity", Gen. Relativ. Gravit., 29, 307-343, (1997). 10, 14, 10.2.1, 10.2.2

[238] Kijowski, J., "A consistent canonical approach to gravitational energy", in Ferrarese, G., ed., Advances in General Relativity and Cosmology, Proceedings of the International Conference in Memory of A. Lichnerowicz, Isola d'Elba, Italy, 12-15 Jun 2002, 129-145, (Pitagora, Bologna, Italy, 2002). 10.2.2

[239] Kijowski, J., and Tulczyjew, W.M., A Symplectic Framework for Field Theories, vol. 107 of Lecture Notes in Physics, (Springer, Berlin, Germany; New York, U.S.A., 1979). 10.2.1, $18,11.3 .1$

[240] Koc, P., and Malec, E., "Trapped surfaces in nonspherical open universes", Acta Phys. Pol. $B, \mathbf{2 3}, 123-133,(1992) . \quad 13.2 .2$

[241] Kodama, H., "Conserved energy flux for the spherically symmetric system and the backreaction problem in the black hole evaporation", Prog. Theor. Phys., 63, 1217-1228, (1980). 4

[242] Komar, A., "Covariant conservation laws in general relativity", Phys. Rev., 113, 934-936, (1959). 3.1 .5

[243] Kramer, D., Stephani, H., MacCallum, M.A.H., and Herlt, E., Exact Solutions of Einstein's Field Equations, Cambridge Monographs on Mathematical Physics, (Cambridge University Press, Cambridge, U.K.; New York, U.S.A., 1980). 4.2 .5

[244] Kulkarni, R., Chellathurai, V., and Dadhich, N., "The effective mass of the Kerr spacetime", Class. Quantum Grav., 5, 1443-1445, (1988). 12.2

[245] Lau, S.R., "Canonical variables and quasi-local energy in general relativity", Class. Quantum Grav., 10, 2379-2399, (1993). Related online version (cited on 29 January 2004):

http://arXiv.org/abs/gr-qc/9307026v3. file corrupt. 10.1.4

[246] Lau, S.R., "Spinors and the reference point of quasi-local energy", Class. Quantum Grav., 12, 1063-1079, (1995). Related online version (cited on 29 January 2004):

http://arXiv.org/abs/gr-qc/9409022v2. 10.1.4

[247] Lau, S.R., "New variables, the gravitational action and boosted quasilocal stress-energymomentum", Class. Quantum Grav., 13, 1509-1540, (1996). Related online version (cited on 29 January 2004):

http://arXiv.org/abs/gr-qc/9504026v3. 10.1.4, 10.1.4

[248] Lau, S.R., "Lightcone reference for total gravitational energy", Phys. Rev. D, 60, 104034-14, (1999). Related online version (cited on 29 January 2004):

http://arXiv.org/abs/gr-qc/9903038. 17, 10.1.7 
[249] Lau, S.R., "Lectures given at the 'International Workshop on Geometric Physics"', unknown status, (2000). Physics and Mathematical Divisions, NCTS, Hsinchu, Taiwan, 24-26 July. $17,10.1 .7$

[250] Lau, S.R., personal communication, (July, 2003). 10.1.7

[251] Lee, J., and Wald, R.M., "Local symmetries and constraints", J. Math. Phys., 31, 725-743, (1990). $\quad 11.3 .1$

[252] Lind, R.W., Messmer, J., and Newman, E.T., "Equations of motion for the sources of asymptotically flat spaces", J. Math. Phys., 13, 1884-1891, (1972). 3.2 .4

[253] Liu, C.-C.M., and Yau, S.-T., "Positivity of quasilocal mass", Phys. Rev. Lett., 90, 2311021-4, (2003). Related online version (cited on 29 January 2004):

http://arXiv.org/abs/gr-qc/0303019v2. 4.1.4, 9, 10, 10.2.2, 10.4.1, 10.4.1, 10.4.2

[254] Liu, C.-C.M., and Yau, S.-T., "Positivity of quasilocal mass II", (2004). URL (cited on 21 February 2005):

http://arXiv.org/abs/math.DG/0412292v2. 10.4 .2

[255] Ludvigsen, M., and Vickers, J.A.G., "The positivity of the Bondi mass", J. Phys. A, 14, L389-L391, (1981). 3.2.3

[256] Ludvigsen, M., and Vickers, J.A.G., "A simple proof of the positivity of the Bondi mass", $J$. Phys. A, 15, L67-L70, (1982). 3.2.3

[257] Ludvigsen, M., and Vickers, J.A.G., "An inequality relating mass and electric charge in general relativity", J. Phys. A, 16, 1169-1174, (1983). 13.2.1

[258] Ludvigsen, M., and Vickers, J.A.G., "An inequality relating total mass and the area of a trapped surface in general relativity", J. Phys. A, 16, 3349-3353, (1983). 13.2 .1

[259] Ludvigsen, M., and Vickers, J.A.G., "Momentum, angular momentum and their quasi-local null surface extensions", J. Phys. A, 16, 1155-1168, (1983). 8.1.1, 8.1.2, 8.1.3, 9.1

[260] Malec, E., "Hoop conjecture and trapped surfaces in non-spherical massive systems", Phys. Rev. Lett., 67, 949-952, (1991). 13.2.2

[261] Malec, E., Mars, M., and Simon, W., "On the Penrose inequality for general horizons", Phys. Rev. Lett., 88, 121102-1-121102-4, (2002). Related online version (cited on 29 January 2004): http://arXiv.org/abs/gr-qc/0201024v2. 10

[262] Malec, E., and Ó Murchadha, N., "Trapped surfaces and the Penrose inequality in spherically symmetric geometries", Phys. Rev. D, 49, 6931-6934, (1994). 4, 13.2.1

[263] Maluf, J.W., "Hamiltonian formulation of the teleparallel description of general relativity", J. Math. Phys., 35, 335-343, (1994). 3.1 .4

[264] Martinez, E.A., "Quasilocal energy for a Kerr black hole", Phys. Rev. D, 50, 4920-4928, (1994). Related online version (cited on 29 January 2004): http://arXiv.org/abs/gr-qc/9405033v2. 10.1.7

[265] Mason, L.J., "A Hamiltonian interpretation of Penrose's quasi-local mass", Class. Quantum Grav., 6, L7-L13, (1989). 7.2.2, 7.3.3 
[266] Mason, L.J., and Frauendiener, J., "The Sparling 3-form, Ashtekar variables and quasi-local mass", in Bailey, T.N., and Baston, R.J., eds., Twistors in Mathematics and Physics, vol. 156 of London Mathematical Society Lecture Note Series, 189-217, (Cambridge University Press, Cambridge, U.K.; New York, U.S.A., 1990). 3.2.1, 3.3.1, 7.2.2, 7.3.3, 11.3.4

[267] Miao, P., "On existence of static metric extensions in general relativity", Commun. Math. Phys., 241, 27-46, (2003). Related online version (cited on 29 January 2004): http://arXiv.org/abs/math-ph/0309041v1. 5.1 .3

[268] Misner, C.W., and Sharp, D.H., "Relativistic Equations for Adiabatic, Spherically Symmetric Gravitational Collapse", Phys. Rev., 136, B 571-B 576, (1964). 4.2.1, 4.2.1

[269] Misner, C.W., Thorne, K.S., and Wheeler, J.A., Gravitation, (W.H. Freeman, San Francisco, U.S.A., 1973). $\quad 13.2 .2$

[270] Møller, C., "On the localization of the energy of a physical system in general theory of relativity", Ann. Phys. (N.Y.), 4, 347-371, (1958). 3.1.2

[271] Møller, C., "Conservation laws and absolute parallelism in general relativity", Mat.-Fys. Skr. K. Danske Vid. Selsk., 1(10), 1-50, (1961). 3.1 .4

[272] Moreschi, O.M., "Unambiguous angular momentum of radiative spacetimes and asymptotic structure in terms of the center of mass system", (2003). URL (cited on 29 January 2004): http://arXiv.org/abs/gr-qc/0305010v1. 3.2.4

[273] Moreschi, O.M., "Intrinsic angular momentum and centre of mass in general relativity", Class. Quantum Grav., 21, 5409-5425, (2004). Related online version (cited on 29 January 2004): http://arXiv.org/abs/gr-qc/0209097v2. 3.2.4

[274] Moreschi, O.M., and Sparling, G.A.J., "On the positive energy theorem involving mass and electromagnetic charges", Commun. Math. Phys., 95, 113-120, (1984). 13.2.1

[275] Mukohyama, S., and Hayward, S.A., "Quasi-local first law of black hole mechanics", Class. Quantum Grav., 17, 2153-2157, (2000). Related online version (cited on 29 January 2004): http://arXiv.org/abs/gr-qc/9905085v2. 13.3 .1

[276] Nadirashvili, N., and Yuan, Y., "Counterexamples for local isometric embedding", (2002). URL (cited on 29 January 2004): http://arXiv.org/abs/math.DG/0208127v1. 17

[277] Nahmad-Achar, E., "Is gravitational field energy density well defined for static, spherically symmetric configurations?", in Blair, D.G., and Buckingham, M.J., eds., The Fifth Marcel Grossmann Meeting on recent developments in theoretical and experimental general relativity, gravitation and relativistic field theories, Proceedings of the meeting held at The University of Western Australia, 8-13 August 1988, 1223-1225, (World Scientific, Singapore; River Edge, U.S.A., 1989). 12.3

[278] Nakao, K., "On a Quasi-Local Energy Outside the Cosmological Horizon", (1995). URL (cited on 29 January 2004): http://arXiv.org/abs/gr-qc/9507022v1. KUNS Preprint KUNS-1352. 4

[279] Nester, J.M., "A new gravitational energy expression with a simple positivity proof", Phys. Lett. A, 83, 241-242, (1981). 3.2 .1 
[280] Nester, J.M., "The gravitational Hamiltonian", in Flaherty, F.J., ed., Asymptotic Behavior of Mass and Spacetime Geometry, Proceedings of the conference, held at Oregon State University, Corvallis, Oregon, USA, October 17-21, 1983, vol. 202 of Lecture Notes in Physics, 155-163, (Springer, Berlin, Germany; New York, U.S.A., 1984). 3.2.1, 11.3.1, 11.3.2

[281] Nester, J.M., "A gauge condition for orthonormal three-frames", J. Math. Phys., 30, 624$626,(1989) . \quad 3.1 .4$

[282] Nester, J.M., “A positive gravitational energy proof”, Phys. Lett. A, 139, 112-114, (1989). $3.1 .4,3.2 .1$

[283] Nester, J.M., "A covariant Hamiltonian for gravity theories", Mod. Phys. Lett. A, 6, 26552661, (1991). $\quad 11.3 .1,11.3 .2$

[284] Nester, J.M., "Special orthonormal frames", J. Math. Phys., 33, 910-913, (1992). 3.1 .4

[285] Nester, J.M., "General pseudotensors and quasilocal quantities", Class. Quantum Grav., 21, S261-S280, (2004). 11.3.2, 11.3.4

[286] Nester, J.M., Ho, F.H., and Chen, C.-M., "Quasi-local center-of-mass for teleparallel gravity", (2004). URL (cited on 21 February 2005): http://arXiv.org/abs/gr-qc/0403101v1. 11.3 .2

[287] Nester, J.M., Meng, F.F., and Chen, C.-M., "Quasi-local center-of-mass", J. Korean Phys. Soc., 45, S22-S25, (2004). Related online version (cited on 21 February 2005): http://arXiv.org/abs/gr-qc/0403103v2. 11.3 .2

[288] Nester, J.M., and Tung, R.-S., "A quadratic spinor Lagrangian for general relativity", Gen. Relativ. Gravit., 27, 115-119, (1995). Related online version (cited on 29 January 2004): http://arXiv.org/abs/gr-qc/9407004. 3.1 .4

[289] Newman, E.T., and Tod, K.P., "Asymptotically flat space-times", in Held, A., ed., General Relativity and Gravitation: One Hundred Years After the Birth of Albert Einstein, vol. 2, 1-36, (Plenum Press, New York, U.S.A., 1980). 3.2, 8

[290] Newman, E.T., and Unti, T.W.J., "Behavior of asymptotically flat empty spaces", J. Math. Phys., 3, 891-901, (1962). 3.2.3, 8

[291] Ó Murchadha, N., "Total energy-momentum in general relativity", J. Math. Phys., 27, 21112128, (1986). 3.2 .1

[292] Ó Murchadha, N., Szabados, L.B., and Tod, K.P., "Comment on "Positivity of Quasilocal Mass"”, Phys. Rev. Lett., 92, 259001-1, (2004). Related online version (cited on 29 January 2004):

http://arXiv.org/abs/gr-qc/0311006v1. 10.2.2, 10.4.2

[293] Page, D.N., "Defining entropy bounds", (2000). URL (cited on 29 January 2004): http://arXiv.org/abs/hep-th/0007238. Report No. Alberta-Thy-09-00. 13.4.1

[294] Page, D.N., "Huge Violations of Bekenstein's Entropy Bound", (2000). URL (cited on 29 January 2004): http://arXiv.org/abs/gr-qc/0005111v1. Report No. Alberta-Thy-06-00. 13.4.1

[295] Page, D.N., "Subsystem entropy exceeding Bekenstein's bound", (2000). URL (cited on 29 January 2004): http://arXiv.org/abs/hep-th/0007237v1. Report No. Alberta-Thy-08-00. 13.4.1 
[296] Park, M.I., "The Hamiltonian dynamics of bounded spacetime and black hole entropy: The canonical method", Nucl. Phys. B, 634, 339-369, (2002). Related online version (cited on 29 January 2004):

http://arXiv.org/abs/hep-th/0111224v4. 21, 13.3.1

[297] Parker, P.E., "On some theorem of Geroch and Stiefel", J. Math. Phys., 25, 597-599, (1984). 10.1.4

[298] Pelath, M.A., Tod, K.P., and Wald, R.M., "Trapped surfaces in prolate collapse in the Gibbons-Penrose construction", Class. Quantum Grav., 15, 3917-3934, (1998). Related online version (cited on 29 January 2004):

http://arXiv.org/abs/gr-qc/9805051v1. 13.2 .2

[299] Pellegrini, C., and Plebanski, J., "Tetrad fields and gravitational fields", Mat.-Fys. Skr. K. Danske Vid. Selsk., 2(4), 1-39, (1963). 3.1 .4

[300] Penrose, R., "Asymptotic properties of fields and space-times", Phys. Rev. Lett., 10, 66-68, (1963). $\quad 4.2 .4$

[301] Penrose, R., "Conformal treatment of infinity", in DeWitt, C., and DeWitt, B.S., eds., Relativity, Groups and Topology, Lectures delivered at Les Houches during the 1963 session of the Summer School of Theoretical Physics, University of Grenoble, 564-584, (Gordon and Breach, New York, U.S.A., 1964). 4.2 .4

[302] Penrose, R., "Zero rest-mass fields including gravitation: asymptotic behaviour", Proc. R. Soc. London, Ser. A, 248, 159-203, (1965). 4.2 .4

[303] Penrose, R., "Gravitational collapse: the role of general relativity", Riv. Nuovo Cimento, 1, 252-276, (1969). reprinted in Gen. Relativ. Gravit. 34 (2002) 1141. 13.2.1

[304] Penrose, R., Techniques of Differential Topology in Relativity, vol. 7 of Regional Conference Series in Applied Mathematics, (SIAM, Philadelphia, U.S.A., 1972). 8.1 .2

[305] Penrose, R., "Naked singularities", Ann. N.Y. Acad. Sci., 224, 125-134, (1973). 4.2.5, 13.2.1

[306] Penrose, R., "Null hypersurface initial data for classical fields of arbitrary spin and for general relativity", Gen. Relativ. Gravit., 12, 225-264, (1980). 11.2.1

[307] Penrose, R., "Quasi-local mass and angular momentum in general relativity", Proc. R. Soc. London, Ser. A, 381, 53-63, (1982). 11, 7.1.2, 7.2.1, 7.2.2, 7.2.3, 7.2.4

[308] Penrose, R., "Mass in general relativity", in Willmore, T.J., and Hitchin, N., eds., Global Riemannian Geometry, 203-213, (Ellis Horwood; Halsted Press, Chichester, U.K.; New York, U.S.A., 1984). 11, 7.2 .1

[309] Penrose, R., "New improved quasi-local mass and the Schwarzschild solutions", Twistor Newsletter, 1984(18), 7-11, (1984). 7.3, 7.3.1

[310] Penrose, R., "A suggested further modification to the quasi-local formula", Twistor Newsletter, $\mathbf{1 9 8 5}(20), 7,(1985)$. $\quad 7.3 .2$

[311] Penrose, R., "Aspects of quasi-local angular momentum", in Isenberg, J.A., ed., Mathematics and General Relativity, Proceedings of the AMS-IMS-SIAM Joint Summer Research Conference held June 22-28, 1986, vol. 71 of Contemporary Mathematics, 1-8, (American Mathematical Society, Providence, U.S.A., 1988). 7.2.3 
[312] Penrose, R., and Rindler, W., Spinors and space-time. Vol.1: Two-spinor calculus and relativistic fields, Cambridge Monographs on Mathematical Physics, (Cambridge University Press, Cambridge, U.K.; New York, U.S.A., 1984). 2, 4.1, 4.1.4, 4.1.6, 8, 10, 7

[313] Penrose, R., and Rindler, W., Spinors and space-time. Vol.2: Spinor and twistor methods in space-time geometry, Cambridge Monographs on Mathematical Physics, (Cambridge University Press, Cambridge, U.K.; New York, U.S.A., 1986). 3.2, 3.2.1, 3.2.3, 4.1, 4.2.4, 8, 4.2.5, $7,11,7.1 .2,7.2 .2,7.2 .3,7.2 .4,7.3 .1$

[314] Perry, M.J., "The positive energy theorem and black holes", in Flaherty, F.J., ed., Asymptotic Behavior of Mass and Spacetime Geometry, Proceedings of the conference, held at Oregon State University, Corvallis, Oregon, USA, October 17-21, 1983, vol. 202 of Lecture Notes in Physics, 31-40, (Springer, Berlin, Germany; New York, U.S.A., 1984). 3.2, 3.2 .1

[315] Petrov, A.N., and Katz, J., "Conservation laws for large perturbations on curved backgrounds", in Frere, J.M., Henneaux, M., Servin, A., and Spindel, P., eds., Fundamental Interactions: From Symmetries to Black Holes, Proceedings of the conference held 24-27 March 1999 at the Université Libre de Bruxelles, Belgium, 147-157, (Université Libre de Bruxelles, Brussels, Belgium, 1999). Related online version (cited on 29 January 2004): http://arXiv .org/abs/gr-qc/9905088v1. 3.1.3

[316] Petrov, A.N., and Katz, J., "Relativistic conservation laws on curved backgrounds and the theory of cosmological perturbations", Proc. R. Soc. London, 458, 319-337, (2002). Related online version (cited on 29 January 2004):

http://arXiv.org/abs/gr-qc/9911025v3. 3.1.2, 3

[317] Pons, J.M., "Boundary conditions from boundary terms, Noether charges and the trace K Lagrangian in general relativity", Gen. Relativ. Gravit., 35, 147-174, (2003). Related online version (cited on 21 February 2005): http://arXiv.org/abs/gr-qc/0105032v3. 14

[318] Purdue, P., "The gauge invariance of general relativistic tidal heating", Phys. Rev. D, 60, 104054-1-8, (1999). Related online version (cited on 29 January 2004): http://arXiv.org/abs/gr-qc/9901086v2. 13.1

[319] Regge, T., and Teitelboim, C., "Role of surface integrals in the Hamiltonian formulation of general relativity", Ann. Phys. (N.Y.), 88, 286-318, (1974). 3.2.1, 3.2.2, 3.3.3, 11

[320] Reula, O., "Existence theorem for solutions of Witten's equation and nonnegativity of total mass", J. Math. Phys., 23, 810-814, (1982). 3.2.1

[321] Reula, O., and Tod, K.P., "Positivity of the Bondi energy", J. Math. Phys., 25, 1004-1008, (1984). $\quad 3.2 .1,3.2 .3$

[322] Rosen, N., "Localization of gravitational energy", Found. Phys., 15, 997-1008, (1986). 3.1 .3

[323] Rosenfeld, L., "Sur le tenseur d'impulsion-énergie", Mem. R. Acad. Belg., Cl. Sci., 18(6), (1940). 2.1.2, 2

[324] Rovelli, C., "What is observable is classical and quantum physics?", Class. Quantum Grav., 8, 297-316, (1991). 3.3.1

[325] Sachs, R.K., "Asymptotic symmetries in gravitational theory", Phys. Rev., 125, 2851-2864, (1962). $\quad 3.2 .3$

Living Reviews in Relativity

http: //www . livingreviews . org/lrr-2004-4 
[326] Sachs, R.K., "On the characteristic initial value problem in gravitational theory", J. Math. Phys., 3, 908-914, (1962). 11.2.1

[327] Saharian, A.A., "Energy-momentum tensor for a scalar field on manifolds with boundaries", Phys. Rev. D, 69, 085005-1-16, (2004). Related online version (cited on 29 January 2004): http://arXiv.org/abs/hep-th/0308108v2. 1

[328] Schoen, R., and Yau, S.-T., "Positivity of the total mass of a general space-time", Phys. Rev. Lett., 43, 1457-1459, (1979). 3.2.1, 6.2.2

[329] Schoen, R., and Yau, S.-T., "Proof of the positive mass theorem. II", Commun. Math. Phys., 79, 231-260, (1981). 3.2 .1

[330] Schoen, R., and Yau, S.-T., "Proof that the Bondi mass is positive", Phys. Rev. Lett., 48, 369-371, (1982). $\quad 3.2 .3$

[331] Sen, A., "On the existence of neutrino 'zero-modes' in vacuum spacetimes", J. Math. Phys., 22, 1781-1786, (1981). 4.1 .2

[332] Senovilla, J.M.M., "Super-energy tensors", Class. Quantum Grav., 17, 2799-2842, (2000). Related online version (cited on 29 January 2004):

http://arXiv.org/abs/gr-qc/9906087v1. 2

[333] Senovilla, J.M.M., "(Super) $)^{n}$-energy for arbitrary fields and its interchange: Conserved quantities", Mod. Phys. Lett. A, 15, 159-166, (2000). Related online version (cited on 29 January 2004):

http://arXiv.org/abs/gr-qc/9905057v1. 2

[334] Shaw, W.T., "Spinor fields at spacelike infinity", Gen. Relativ. Gravit., 15, 1163-1189, (1983). $\quad 7.2 .4$

[335] Shaw, W.T., "Twistor theory and the energy-momentum and angular momentum of the gravitational field at spatial infinity", Proc. R. Soc. London, Ser. A, 390, 191-215, (1983). 7.2 .4

[336] Shaw, W.T., "Symplectic geometry of null infinity and two-surface twistors", Class. Quantum Grav., 1, L33-L37, (1984). 7.2.4

[337] Shaw, W.T., "Twistors, asymptotic symmetries and conservation laws at null and spatial infinity", in Flaherty, F.J., ed., Asymptotic Behavior of Mass and Spacetime Geometry, Proceedings of the conference, held at Oregon State University, Corvallis, Oregon, USA, October 17-21, 1983, vol. 202 of Lecture Notes in Physics, 165-176, (Springer, Berlin, Germany; New York, U.S.A., 1984). 7.2 .4

[338] Shaw, W.T., "The asymptopia of quasi-local mass and momentum: I. General formalism and stationary spacetimes", Class. Quantum Grav., 3, 1069-1104, (1986). 8, 6.1.2, 6.3, 7.2.4, 8.1 .3

[339] Shaw, W.T., "Total angular momentum for asymptotically flat spacetimes with nonvanishing stress tensor", Class. Quantum Grav., 3, L77-L81, (1986). 7.2.4

[340] Shaw, W.T., "Quasi-local mass for 'large' spheres", in Isenberg, J.A., ed., Mathematics and General Relativity, Proceedings of the AMS-IMS-SIAM Joint Summer Research Conference held June 22-28, 1986, vol. 71 of Contemporary Mathematics, 15-22, (American Mathematical Society, Providence, U.S.A., 1988). 6.1.2, 7.2.4, 8.1.3 
[341] Shi, Y., and Tam, L.-F., "Positive mass theorem and the boundary behaviors of compact manifolds with nonnegative scalar curvature", J. Differ. Geom., 62, 79-125, (2002). Related online version (cited on 29 January 2004):

http://arxiv.org/abs/math.DG/0301047v1. 10.1.7, 10.4.2

[342] Shi, Y., and Tam, L.-F., "Some lower estimates of ADM mass and Brown-York mass", (2004). URL (cited on 21 February 2005): http://arXiv.org/abs/math.DG/0406559v3. 10.1.7

[343] Smarr, L.L., "Surface geometry of charged rotating black holes", Phys. Rev. D, 7, 289-295, (1973). 17, 10.1.7

[344] Sommers, P., "The geometry of the gravitational field at spacelike infinity", J. Math. Phys., 19, 549-554, (1978). 3.2.1, 7.2.4

[345] Sparling, G.A.J., "Twistors, spinors and the Einstein vacuum equations", unknown status, (1982). University of Pittsburgh Preprint. 3.2.1, 3.3.1, 11.3.1

[346] Spivak, M., A Comprehensive Introduction to Differential Geometry, vol. 5, (Publish or Perish, Berkeley, U.S.A., 1979), 2nd edition. 17

[347] Stewart, J.M., Advanced general relativity, Cambridge Monographs on Mathematical Physics, (Cambridge University Press, Cambridge, U.K.; New York, U.S.A., 1990). 4.1

[348] Stewart, J.M., and Friedrich, H., "Numerical relativity. I. The characteristic initial value problem", Proc. R. Soc. London, Ser. A, 384, 427-454, (1982). 11.2.1

[349] Streubel, M., "Conserved" quantities for isolated gravitational systems", Gen. Relativ. Gravit., 9, 551-561, (1978). 4.2.5, 7.1.1

[350] Susskind, L., "The world as a hologram", J. Math. Phys., 36, 6377-6396, (1995). Related online version (cited on 29 January 2004):

http://arXiv.org/abs/hep-th/9409089v2. 13.4 .2

[351] Szabados, L.B., "Commutation properties of cyclic and null Killing symmetries", J. Math. Phys., 28, 2688-2691, (1987). 4.2 .5

[352] Szabados, L.B., "Canonical pseudotensors, Sparling's form and Noether currents", unknown status, (1991). KFKI Report 1991-29/B. 2, 3.1.2, 3, 3.1.4, 3.3.1, 11.3.4

[353] Szabados, L.B., "On canonical pseudotensors, Sparling's form and Noether currents", Class. Quantum Grav., 9, 2521-2541, (1992). 3.1.2, 3, 3.3.1, 11.3.4

[354] Szabados, L.B., "On the positivity of the quasi-local mass", Class. Quantum Grav., 10, 1899-1905, (1993). 2.2, 2.2.3, 8.1.3, 8.2.1, 8.2.3, 8.2.3, 9.2, 14.1

[355] Szabados, L.B., "Two dimensional Sen connections", in Kerr, R.P., and Perjés, Z., eds., Relativity Today, Proceedings of the Fourth Hungarian Relativity Workshop, July 12-17, 1992, Gárdony, 63-68, (Akadémiai Kiadó, Budapest, Hungary, 1994). 4.1

[356] Szabados, L.B., "Two dimensional Sen connections and quasi-local energy-momentum", Class. Quantum Grav., 11, 1847-1866, (1994). Related online version (cited on 29 January 2004):

http://arXiv.org/abs/gr-qc/9402005v1. 4.1.6, 4.1.7, 7.2.2, 7.2.3, 8.2.1, 8.2.3, 8.2.3, 8.2.4, 9.2

Living Reviews in Relativity

http: //www . livingreviews . org/lrr-2004-4 
[357] Szabados, L.B., "Two dimensional Sen connections in general relativity", Class. Quantum Grav., 11, 1833-1846, (1994). Related online version (cited on 29 January 2004):

http://arXiv.org/abs/gr-qc/9402001v1. 4.1

[358] Szabados, L.B., "Quasi-local energy-momentum and two-surface characterization of the ppwave spacetimes", Class. Quantum Grav., 13, 1661-1678, (1996). Related online version (cited on 29 January 2004):

http://arXiv.org/abs/gr-qc/9512013v1. 2.2, 2.2.3, 2.2.5, 4.1, 4.1.3, 9.2, 13.5

[359] Szabados, L.B., "Quasi-local energy-momentum and the Sen geometry of two-surfaces", in Chruściel, P.T., ed., Mathematics of Gravitation, Part I: Lorentzian Geometry and Einstein Equations, vol. 41 of Banach Center Publications, 205-219, (Polish Academy of Sciences, Institute of Mathematics, Warsaw, Poland, 1997). 4.1

[360] Szabados, L.B., "On certain quasi-local spin-angular momentum expressions for small spheres", Class. Quantum Grav., 16, 2889-2904, (1999). Related online version (cited on 29 January 2004):

http://arXiv.org/abs/gr-qc/9901068v1. 6, 8.1.3, 12, 9, 9.1, 9.2

[361] Szabados, L.B., "On certain global conformal invariants and 3-surface twistors of initial data sets", Class. Quantum Grav., 17, 793-811, (2000). Related online version (cited on 29 January 2004):

http://arXiv.org/abs/gr-qc/9909052v1. 7.2 .5

[362] Szabados, L.B., "Quasi-local energy-momentum and angular momentum in general relativity I.: The covariant Lagrangian approach", unknown status, (2000). 8

[363] Szabados, L.B., "On certain quasi-local spin-angular momentum expressions for large spheres near the null infinity", Class. Quantum Grav., 18, 5487-5510, (2001). Related online version (cited on 29 January 2004):

http://arxiv.org/abs/gr-qc/0109047. Corrigendum: Class. Quantum Grav. 192333 (2002). $\quad 3.2 .3,3.2 .4,4.1 .7,8,8,7.2 .3,9,9.2,9.2,13.5$

[364] Szabados, L.B., "On the roots of the Poincaré structure of asymptotically flat spacetimes", Class. Quantum Grav., 20, 2627-2661, (2003). Related online version (cited on 29 January 2004):

http://arXiv.org/abs/gr-qc/0302033v2. 2.2.4, 3.2.2, 21

[365] Szabados, L.B., "Quasi-local holography and quasi-local mass of classical fields in Minkowski spacetime", Class. Quantum Grav., 22, 855-878, (2005). Related online version (cited on 21 February 2005):

http://arXiv.org/abs/gr-qc/0411148v2. 2.2.5, 8.2.3, 13.4.2

[366] 't Hooft, G., "Dimensional Reduction in Quantum Gravity", (1993). URL (cited on 29 January 2004):

http://arXiv.org/abs/gr-qc/9310026v1. Utrecht preprint THU-93/26. 13.4.2

[367] Thorne, K.S., "Nonspherical gravitational collapse, a short review", in Klauder, J., ed., Magic Without Magic: John Archibald Wheeler. A Collection of Essays in Honor of His Sixtieth Birthday, 231-258, (W.H. Freeman, San Francisco, U.S.A., 1972). 13.2 .2

[368] Tipler, F.J., "Penrose's quasi-local mass in the Kantowski-Sachs closed universe", Class. Quantum Grav., 2, L99-L103, (1985). 7.2.5 
[369] Tod, K.P., "All metrics admitting super-covariantly constant spinors", Phys. Lett. B, 121, 241-244, (1983). 13.2.1

[370] Tod, K.P., "Quasi-local charges in Yang-Mills theory", Proc. R. Soc. London, Ser. A, 389, 369-377, (1983). $\quad 7.2 .1$

[371] Tod, K.P., "Some examples of Penrose's quasi-local mass construction", Proc. R. Soc. London, Ser. A, 388, 457-477, (1983). 7.1.2, 7.2.3, 7.2.4, 7.2.5, 12.1

[372] Tod, K.P., "More on quasi-local mass", Twistor Newsletter(18), 3-6, (1984). 7.2.6, 7.2.6, 7.3.1

[373] Tod, K.P., "Three-surface twistors and conformal embedding", Gen. Relativ. Gravit., 16, 435-443, (1984). 7.2 .5

[374] Tod, K.P., "Penrose's quasi-local mass and the isoperimetric inequality for static black holes", Class. Quantum Grav., 2, L65-L68, (1985). 7.2.5, 13.2.1

[375] Tod, K.P., "More on Penrose's quasilocal mass", Class. Quantum Grav., 3, 1169-1189, (1986). $\quad 4.1,7.2 .3,7.2 .5,13.2 .1$

[376] Tod, K.P., "Quasi-local mass and cosmological singularities", Class. Quantum Grav., 4, 1457-1468, (1987). 7.2 .5

[377] Tod, K.P., "Penrose's quasi-local mass", in Bailey, T.N., and Baston, R.J., eds., Twistors in Mathematics and Physics, vol. 156 of London Mathematical Society Lecture Note Series, 164-188, (Cambridge University Press, Cambridge, U.K.; New York, U.S.A., 1990). 7 , 7.2.2, 7.2.3, 7.2.4, 7.2.5, 7.3, 7.3.1, 7.3.2

[378] Tod, K.P., "Penrose's quasi-local mass and cylindrically symmetric spacetimes", Class. Quantum Grav., 7, 2237-2266, (1990). 7.2.5

[379] Tod, K.P., "The hoop conjecture and the Gibbons-Penrose construction of trapped surfaces", Class. Quantum Grav., 9, 1581-1591, (1992). 13.2 .2

[380] Tod, K.P., "The Stützfunktion and the cut function", in Janis, A.I., and Porter, J.R., eds., Recent Advances in General Relativity: Essays in honor of Ted Newman, Papers from the Discussion Conference on Recent Advances in General Relativity, held at the University of Pittsburgh, May 3-5, 1990, vol. 4 of Einstein Studies, 182-195, (Birkhäuser, Boston, U.S.A., 1992). $\quad 4.1,4.1 .3,10.4 .2$

[381] Torre, C.G., "Null surface geometrodynamics", Class. Quantum Grav., 3, 773-791, (1986). 11.2.1

[382] Trautman, A., "Conservation laws in general relativity", in Witten, L., ed., Gravitation: An Introduction to Current Research, 169-198, (Wiley, New York, U.S.A., 1962). 3.1.2, 3, 4.2.5

[383] Tung, R.-S., and Nester, J.M., "The quadratic spinor Lagrangian is equivalent to the teleparallel theory", Phys. Rev. D, 60, 021501-1-5, (1999). Related online version (cited on 29 January 2004): http://arXiv .org/abs/gr-qc/9809030v2. 3.1.4

[384] Tung, R.S., and Jacobson, T., "Spinor 1-forms as gravitational potentials", Class. Quantum Grav., 12, L51-L55, (1995). Related online version (cited on 21 February 2005): http://arXiv .org/abs/gr-qc/9502037v1. 3.1.4 
[385] Unruh, W.G., and Wald, R.M., "Acceleration radiation and the generalized second law of thermodynamics", Phys. Rev. D, 25, 942-958, (1982). 13.4.1

[386] Utiyama, R., "Invariant theoretical interpretation of interactions", Phys. Rev., 101, 15971607, (1956). 3.1 .4

[387] Wald, R.M., General Relativity, (University of Chicago Press, Chicago, U.S.A., 1984). 2.2.2, $3.2 .2,4.2 .5,14,12.1,13.3 .1$

[388] Wald, R.M., "On identically closed forms locally constructed from a field", J. Math. Phys., 31, 2378-2384, (1990). 2.2.1, 11.3.1

[389] Wald, R.M., "Black hole entropy is Noether charge", Phys. Rev. D, 48, 3427-3431, (1993). Related online version (cited on 29 January 2004):

http://arXiv.org/abs/gr-qc/9307038v1. 2.1.2, 11.3.1, 13.3.1

[390] Wald, R.M., Quantum Field Theory in Curved Spacetime and Black Hole Thermodynamics, Chicago Lectures in Physics, (University of Chicago Press, Chicago, U.S.A., 1994). 13.3.1, 13.4.1

[391] Wald, R.M., "Gravitational Collapse and Cosmic Censorship", (1997). URL (cited on 29 January 2004):

http://arXiv.org/abs/gr-qc/9710068v3. EFI Preprint No. 97-43. 13.2.2

[392] Wald, R.M., "The Thermodynamics of Black Holes", Living Rev. Relativity, 4, lrr-2001-6, (2001). URL (cited on 29 January 2004):

http://www.livingreviews.org/lrr-2001-6. 13.3.1, 13.4.2

[393] Weinstein, G., and Yamada, S., "On a Penrose inequality with charge", (2004). URL (cited on 21 February 2005):

http://arXiv.org/abs/math.DG/0405602v3. 13.2.1

[394] Winicour, J., "Angular momentum in general relativity", in Held, A., ed., General Relativity and Gravitation: One Hundred Years After the Birth of Albert Einstein, vol. 2, 71-96, (Plenum Press, New York, U.S.A., 1980). 3.2, 3.2.3

[395] Winicour, J.H., and Tamburino, L., "Lorentz-covariant gravitational energy-momentum linkages", Phys. Rev. Lett., 15, 601-605, (1965). 3.2.3, 3.2.3, 3.2.4

[396] Wipf, A., "Hamilton's formalism for systems with constraints", in Ehlers, J., and Friedrich, H., eds., Canonical Gravity: From Classical to Quantum, Proceedings of the 117th WE Heraeus Seminar, Bad Honnef, Germany, 13-17 September 1993, vol. 434 of Lecture Notes in Physics, 22-58, (Springer, Berlin, Germany; New York, U.S.A., 1994). 2.2.2, 4.3.1

[397] Witten, E., "A new proof of the positive energy theorem", Commun. Math. Phys., 30, 381402, (1981). $\quad 3.2 .1$

[398] Woodhouse, N.M.J., "Ambiguities in the definition of quasi-local mass", Class. Quantum Grav., 4, L121-L123, (1987). 7.2.6, 7.3.1

[399] Yau, S.-T., "Geometry of three manifolds and existence of black holes due to boundary effect", Adv. Theor. Math. Phys., 5, 755-767, (2001). Related online version (cited on 29 January 2004):

http://arxiv.org/abs/math.DG/0109053v2. 10.4.2 
[400] Yoon, J.H., "Quasi-local energy conservation law derived from the Einstein's equations", (1998). URL (cited on 29 January 2004):

http://arXiv.org/abs/gr-qc/9806078v1. 11.2 .2

[401] Yoon, J.H., "Quasi-local energy for general spacetimes", J. Korean Phys. Soc., 34, 108-111, (1999). 11.2.2

[402] Yoon, J.H., "Quasi-local conservation equations in general relativity", Phys. Lett. A, 292, 166-172, (2001). Related online version (cited on 29 January 2004):

http://arXiv.org/abs/gr-qc/0004074v2. 11.2 .2

[403] Yoon, J.H., "(1+1)-dimensional formalism and quasi-local conservation equations", in Ferrarese, G., ed., Advances in General Relativity and Cosmology, Proceedings of the International Conference in Memory of A. Lichnerowicz, Isola d'Elba, Italy, 12-15 Jun 2002, (Pitagora, Bologna, Italy, 2002). Related online version (cited on 29 January 2004):

http://www.arxiv.org/abs/gr-qc/0212042v1. 11.2.2

[404] Yoon, J.H., "New Hamiltonian formalism and quasilocal conservation equations of general relativity", Phys. Rev. D, 70, 084037-1-20, (2004). Related online version (cited on 21 February 2005):

http://arXiv.org/abs/gr-qc/0406047v2. 11.2 .2

[405] York, J.W., "Role of conformal three-geometry in the dynamics of gravitation", Phys. Rev. Lett., 28, 1082-1085, (1972). 14

[406] York Jr, J.W., "Boundary terms in the action principles of general relativity", Found. Phys., 16, 249-257, (1986). 14

[407] Yoshino, H., Nambu, Y., and Tomimatsu, A., "Hoop conjecture for colliding black holes: Non-time-symmetric initial data", Phys. Rev. D, 65, 064034-1-6, (2002). Related online version (cited on 29 January 2004):

http://arXiv.org/abs/gr-qc/0109016v1. 13.2.2

[408] Zannias, T., "Trapped surfaces on a spherically symmetric initial data set", Phys. Rev. D, 45, 2998-3001, (1992). 4, 13.2.1

[409] Zaslavskii, O.B., "Entropy and action bounds for charged black holes", Gen. Relativ. Gravit., 24, 973-983, (1992). 13.4.1 